\title{
MOLECULAR ADAPTATIONS OF MAMMALIAN HIBERNATION: ROLES OF METABOLIC SIGNALING REGULATION IN THE TORPOR-AROUSAL CYCLE
}

by

\section{Cheng-Wei Wu}

B.Sc. Carleton University, 2009

A Thesis Submitted to the Faculty of Graduate Studies and Research in partial fulfillment of the requirements for the degree of

Doctor of Philosophy

in

Biology

Carleton University

Ottawa, Ontario, Canada

(C) Copyright 2014

Cheng-Wei Wu 


\begin{abstract}
For many small mammals, survival over the winter months is a serious challenge because of low environmental temperatures and limited food availability. The solution for thirteen-lined ground squirrels (Ictidomys tridecemlineatus) is hibernation, a metabolically re-programmed state that is characterized by seasonal heterothermy and entry into long periods of torpor. Although many studies have defined the physiological responses of hibernation, including drastic reductions in heart rate, respiration, and body temperature, the regulation of such phenotypic plasticity has yet to be fully characterized at the molecular level. As part of hibernation, metabolic rate is suppressed during torpor to achieve major energy savings through coordinated suppression of non-essential ATPcostly processes. The present thesis examined the role of cell signaling cascades in the regulation of energy dependent cellular processes over the torpor-arousal cycles of hibernation.
\end{abstract}

The insulin signaling pathway, which functions as the regulator of many progrowth processes such as protein synthesis, was shown to be regulated during torpor. Significant inhibition of this pathway was most evident in skeletal muscle but not in cardiac muscle during torpor. This inhibition was characterized by reduced phosphorylation of mammalian target of rapamycin kinase (mTOR), which led to subsequent inhibition of various proteins involved in ribosome assembly that are required for protein translation. The cell cycle, another energy dependent metabolic process, was also strongly inhibited during torpor. Cell cycle arrest was evident in liver (proliferative capable) but not in skeletal muscle (terminally differentiated), through mechanisms 
similar to those observed in $\mathrm{G}_{1}$ and $\mathrm{G}_{1} / \mathrm{S}$ arrest. The observed cell cycle arrest was characterized by down-regulation of proteins cyclin $\mathrm{D}$ and cyclin $\mathrm{E}$ which function as positive regulators of cell cycle progression, and up-regulation of the cell cycle inhibitors (CKIs) p15INK4b and p21CIP1. Up-regulation of CKIs during torpor was linked to members of the Smad family of transcription factors, which were activated during torpor, including increased nuclear inclusion and DNA binding activity of Smad 3. Overall, the data presented in this thesis document molecular mechanisms that function to reduce cellgrowth and proliferation during torpor, via inhibition of protein synthesis and cell cycle progression. 


\section{ACKNOWLEDGEMENT}

The work presented in this thesis, although requiring countless hours of effort and energy, would not have been possible without the support and opportunities given to me by Dr. Ken Storey. The guidance, influence, support, and patience Dr. Storey and Jan Storey provided for the last 6 years help shaped me to become both the person and the scientist that I am today, which has led to many opportunities that continue to guide me towards the next stage of my scientific career, and for that, I am forever grateful. I am also grateful for the help and guidance provided to me by my thesis advisors Dr. Walsh and Dr. Ianoul, both of whom showed continuous support of my research; and special thanks to Dr. Walsh for his guidance and mentorship in my post-doctoral search.

As we are often considered to be a product of our environment, I would also like to take this opportunity to thank all my previous mentors and current colleagues in the Storey lab. Although too many to mention, special thanks go to Kyle Biggar and Ryan Bell for taking part in collaborative projects, many of which have and will lead to great scientific publications; as well thanks go to Oscar Aguilar for introducing me to the Storey lab.

Most importantly, I would like to thank my parents Tony and Teresa for their unconditional love and support, along with the personal sacrifices they made in their careers to provide my sister and me with the education we have today. As well, a big thank you to my better half Helen, whose support and love has brought out the best in me, and is the continuous driving force of my life.

"Anyone who has never made a mistake has never tried anything new." - Albert Einstein 


\section{TABLE OF CONTENTS}

Title Page

$\begin{array}{ll}\text { Abstract } & \text { ii }\end{array}$

Acknowledgements

Table of Contents $\quad$ V

List of

Vi

Abbreviations

List of Figures

ix

List of Tables $\quad$ xiv

List of Appendices $\quad$ xiv

Chapter $1 \quad$ General Introduction 1

$\begin{array}{lll}\text { Chapter } 2 \text { Materials and Methods } & 20\end{array}$

Chapter 3 Regulation of mTOR signaling network in hibernating 38 ground squirrels

Chapter $4 \quad$ Post-translational regulation of PTEN phosphatase 63

Chapter $5 \quad$ Smad mediated transcriptional response during 93 hibernation

$\begin{array}{lll}\text { Chapter } 6 & \text { Regulation of cell cycle progression in hibernating } & 146\end{array}$

$\begin{array}{lll}\text { Chapter } 7 & \text { General Discussion } & 188\end{array}$

$\begin{array}{ll}\text { Appendices } & 204\end{array}$

$\begin{array}{ll}\text { References } & 215\end{array}$ 


\section{LIST OF ABBREVIATION}

4E-BP1

5'TOP

AA

$\mathrm{AMH}$

AMP, ADP, ATP

AMPK

ANOVA

APS

ATF-2

$\beta$-GP

BLAST

BMP

cAMP

$\mathrm{Cdc} 25$

$\mathrm{Cdk}$

cDNA

cGMP

Chk

CKI

DAF

DAP-kinase

DEPC

DMP

dNTP

DTT

EA

EC

ECL

EDTA

EGTA

eIF2B

eIF4E

ELISA

EMSA

EN

ERK
Eukaryotic translation initiation factor 4E-binding protein 1

5 ' terminal oligopyrimidine tract

Amino acid

Anti-Mullerian hormone

Adenosine mono-, di-, or triphosphate

AMP activated protein kinase

Analysis of variance

Ammonium persulphate

Activating transcription factor 2

$\beta$-glycerol phosphate

Basic local alignment search tool

Bone morphogenetic protein

3', 5'-cyclic adenosine monophosphate

Cell division cycle 25

Cyclin dependent kinase

Complementary deoxyribonucleic acid

Cyclic guanosine monophosphate

Check point kinase

Cyclin-dependent kinase inhibitor

Dauer formation

Death associating protein-kinase

Diethylpyrocarbonate

Dimethylpimelimidate

deoxynucleotide triphosphate

Dithiolthreitol

Early arousal

Euthermic control

Enhanced chemiluminescence

Ethylenediamine tetraacetic acid

Ethylene glycol tetraacetic acid

Eukaryotic initiation factor $2 \mathrm{~B}$

Eukaryotic initiation factor $4 \mathrm{E}$

Enzyme-linked immunosorbent assay

Electrophoretic mobility shift assay

Entry into torpor

Extracellular-signal-regulated kinases 


\begin{tabular}{|c|c|}
\hline ET & Early torpor \\
\hline ETC & Electron transport chain \\
\hline FABP & Fatty-acid-binding protein \\
\hline FoxO & Forkhead box, class $\mathrm{O}$ protein \\
\hline Gadd45- $\beta$ & Growth arrest and DNA-damage-inducible, beta \\
\hline GAPDH & Glyceraldehyde 3-phosphate dehydrogenase \\
\hline GATA & Erythroid transcription factor \\
\hline $\mathrm{G} \beta \mathrm{L}$ & $\mathrm{G}$ protein beta subunit like protein \\
\hline GDP & Guanosine diphosphate \\
\hline GLUT-4 & Glucose transporter type 4 \\
\hline GTP & Guanosine triphosphate \\
\hline HEPES & N- (2-hydroxyethyl) piperazine-N'- (2-ethanesulfonic acid) \\
\hline HIF-1a & Hypoxia-inducible factor 1-alpha \\
\hline HRP & Horseradish peroxidase \\
\hline IA & Interbout arousal \\
\hline IgG-HRP & Immunoglobulin G - horseradish peroxidase \\
\hline $\mathrm{kb}$ & Kilobase \\
\hline $\mathrm{kDa}$ & Kilodalton \\
\hline LT & Late torpor \\
\hline MAPK & Mitogen-activated protein kinase \\
\hline MEF-2 & Myocyte enhancer factor-2 \\
\hline MEK & MAP kinase kinase \\
\hline MH1 & MAD homology 1 \\
\hline miRNA & MicroRNA \\
\hline M-MLV & Moloney murine leukemia virus \\
\hline mRNA & Messenger RNA \\
\hline mTOR & Mammalian target of rapamycin \\
\hline $\mathrm{Na}^{+} \mathrm{K}^{+}$-ATPase & Sodium / potassium ATPase \\
\hline NCBI & National center for biotechnology information \\
\hline ND2 & NADH-ubiquinone oxidoreductase chain 2 \\
\hline Nrf2 & Nuclear factor (erythroid-derived 2)-like 2 \\
\hline ORF & Open reading frame \\
\hline p15INK4b & p15 inhibitor of cyclin-dependent kinase $4 b$ \\
\hline p21CIP1 & p21 CDK interacting protein 1 \\
\hline PAGE & Polyacrylamide gel electrophoresis \\
\hline PBD & Polo-box domain \\
\hline PBS & Phosphate-buffered saline \\
\hline PCNA & Proliferating cell nuclear antigen \\
\hline PCR & Polymerase chain reaction \\
\hline
\end{tabular}




$\begin{array}{ll}\text { PDK-1 } & \text { Phosphoinositide-dependent kinase } \\ \text { PDK-4 } & \text { Pyruvate dehydrogenase lipoamide kinase isozyme 4 } \\ \text { PEST } & \text { Proline (P), glutamic acid (E), serine (S), threonine (T) } \\ \text { PI3K } & \text { Phosphoinositide 3-kinase } \\ \text { PIP }_{2} & \text { Phosphatidylinositol 4,5-bisphosphate } \\ \text { PIP }_{3} & \text { Phosphatidyl inositol-(3,4,5)-triphosphate } \\ \text { PMSF } & \text { Phenylmethylsulfonyl fluoride } \\ \text { PP2A } & \text { Protein phosphatase 2 A } \\ \text { PPAR- } \gamma & \text { Peroxisome proliferator-activated receptor-gamma } \\ \text { PRAS40 } & \text { Proline-rich Akt/PKB substrate 40 kDa } \\ \text { Prdx } & \text { Peroxiredoxin } \\ \text { PTEN } & \text { Phosphatase and tensin homolog } \\ \text { PUFA } & \text { Polyunsaturated fatty acid } \\ \text { PVDF } & \text { Polyvinylidine fluoride } \\ \text { Q10 } & \text { Q10 temperature coefficient } \\ \text { RAPTOR } & \text { Regulatory-associated protein of mTOR } \\ \text { ROS } & \text { Reactive oxygen species } \\ \text { RPP } & \text { Reversible protein phosphorylation } \\ \text { RT } & \text { Room temperature }\left(\sim 21^{\circ} \mathrm{C}\right) \\ \text { RT-PCR } & \text { Reverse-transcriptase polymerase chain reaction } \\ \text { SBE } & \text { Smad binding element } \\ \text { SDS } & \text { Sodium dodecyl sulfate } \\ \text { SEM } & \text { Standard error of the mean } \\ \text { Smad } & \text { Small mothers against decapentaplegic } \\ \text { SSXS } & \text { Ser-SerVal/Met-Ser } \\ \text { TAE } & \text { Tris-acetate-ethylenediamine tetraacetic acid buffer } \\ \text { TBST } & \text { Tris-buffered saline containing Tween-20 } \\ \text { TEMED } & \text { N,N,N',N'-tetra methylethylenediamine } \\ \text { TF } & \text { Transcription factor } \\ \text { TGF- } \beta & \text { Transforming growth factor beta } \\ \text { TMB } & 3,3^{\prime}, 5,5^{\prime}-\text { Tetramethylbenzidine } \\ \text { Tris } & \text { Tris (hydroxymethyl) aminomethane } \\ \text { TSC2 } & \text { Tuberous sclerosis 2 } \\ \text { V } & \text { Maximum velocity } \\ & \end{array}$




\section{LIST OF FIGURES}

FIG. 1.1. Pattern of changes in body temperature of a ground squirrel over a one year period showing multiple bouts of torpor over the winter hibernation season.

FIG. 1.2. Stages of the torpor-arousal cycle.

FIG. 1.3. Body temperature and metabolic rate during entry into torpor.

FIG. 3.1 The mTORC1 signaling pathway.

FIG. 3.2. Regulation pattern of mTOR protein in cardiac and skeletal muscle of I. tridecemlineatus as determined by Western immunoblotting.

FIG. 3.3. Regulation pattern of mTOR regulator proteins in skeletal muscle of I. tridecemlineatus as determined by Western immunoblotting.

FIG. 3.4. Regulation pattern of mTOR regulator proteins in cardiac muscle of I. tridecemlineatus as determined by Western immunoblotting.

FIG. 3.5. Regulation pattern of phosphorylated Akt protein in cardiac and skeletal muscle of I. tridecemlineatus as determined by Western immunoblotting.

FIG. 3.6. Regulation pattern of mTOR downstream proteins in skeletal muscle of I. tridecemlineatus as determined by Western immunoblotting.

FIG. 3.7. Regulation pattern of mTOR downstream proteins in cardiac muscle of I. tridecemlineatus as determined by Western immunoblotting.

FIG. 4.1. Regulation pattern of PTEN in skeletal muscle of $I$. tridecemlineatus as determined by Western immunoblotting.

FIG. 4.2. Regulation pattern of PDK-1 in skeletal muscle of $I$. tridecemlineatus as determined by Western immunoblotting.

FIG. 4.3. Transcriptional regulation of $P T E N$ and $P D K-1$ in skeletal muscle of I. tridecemlineatus as determined by RT-PCR.

FIG. 4.4. Partial cDNA sequence of PTEN 
FIG 4.5. PTEN homology alignment

FIG. 4.6. Partial cDNA sequence of $P D K-1$

FIG 4.7. PDK-1 homology alignment.

FIG. 4.8. Michaelis-Menten kinetics of PTEN phosphatase activity from skeletal muscle of I. tridecemlineatus as determined by the malachite green assay.

FIG. 4.9. Elution profile of euthermic control proteins from heparin affinity chromatography.

FIG. 4.10. Analysis of I. tridecemlineatus PTEN structural stability as determined by pulse-proteolysis.

FIG. 4.11. Prediction of PEST motif from the amino acid sequence of $I$. tridecemlineatus PTEN retrieved from ensemble database using the epestfind bioinformatics program.

FIG. 4.12. Mechanism of pulse-proteolysis method

FIG. 4.13. Proposed mechanisms of PTEN regulation between euthermia and torpor.

FIG 5.1. Mechanism of Smad mediated transcriptional response.

FIG. 5.2. Regulation pattern of Smad 4 protein in liver and skeletal muscle of I. tridecemlineatus as determined by Western immunoblotting.

FIG. 5.3. Regulation pattern of Smad 2 protein in liver and skeletal muscle of I. tridecemlineatus as determined by Western immunoblotting.

FIG. 5.4. Regulation pattern of Smad 3 protein in liver and skeletal muscle of I. tridecemlineatus as determined by Western immunoblotting.

FIG. 5.5. Regulation pattern of Smurf 2 protein in liver and skeletal muscle of I. tridecemlineatus as determined by Western immunoblotting.

FIG. 5.6. Regulation pattern of phosphorylated Smad 2 protein (Ser 465/467) in liver and skeletal muscle of I. tridecemlineatus as determined by immunoblotting. 
FIG. 5.7. Regulation pattern of phosphorylated Smad 3 (Ser 423/425) protein in liver and skeletal muscle of $I$. tridecemlineatus as determined by immunoblotting.

FIG. 5.8. Nuclear localization of SMAD proteins in liver and skeletal muscle of I. tridecemlineatus as determined by immunoblotting.

FIG. 5.9. Transcriptional regulation of Smad2 in liver and skeletal muscle of I. tridecemlineatus as determined by RT-PCR.

FIG. 5.10. Partial cDNA sequence of Smad 2

FIG 5.11. Smad 2 homology alignment.

FIG. 5.12. Transcriptional regulation of $\operatorname{Smad} 3$ in liver and skeletal muscle of I. tridecemlineatus as determined by RT-PCR.

FIG. 5.13. Partial cDNA sequence of Smad 3

FIG 5.14. Smad 3 homology alignment

FIG. 5.15. Transcriptional regulation of Smad 4 in liver and skeletal muscle of I. tridecemlineatus as determined by RT-PCR.

FIG. 5.16. Partial cDNA sequence of Smad 4

FIG 5.17. Smad 3 homology alignment.

FIG. 5.18. Smad3 DNA binding complex in I. tridecemlineatus liver as determined by electrophoretic mobility shift assay (EMSA) on PAGE

FIG. 5.19. DNA binding activity of Smad 3 transcription factor in liver and skeletal muscle of I. tridecemlineatus as determined by enzyme-linked immunosorbent assay (ELISA).

FIG. 5.20. Transcriptional complex of Smad 3 and Smad 4 proteins in liver and skeletal muscle of I. tridecemlineatus as determined by co-immunoprecipitation.

FIG. 5.21. Transcriptional regulation of cell cycle inhibitors in liver of $I$. tridecemlineatus as determined by RT-PCR.

FIG. 5.22. Transcriptional regulation of cell cycle inhibitors in skeletal muscle of I. tridecemlineatus as determined by RT-PCR. 
FIG. 5.23. Partial cDNA sequence of $p 15 I N K 4 b$

FIG 5.24. p15INK4b homology alignment.

FIG. 5.25. Partial cDNA sequence of $p 21 C I P 1$

FIG 5.26. p21CIP1 homology alignment.

FIG. 5.27. Transcriptional regulation of Smad downstream microRNA targets in liver of I. tridecemlineatus as determined by RTPCR.

FIG. 5.28. Transcriptional regulation of Smad downstream microRNA targets in skeletal muscle of I. tridecemlineatus as determined by RT-PCR.

FIG. 6.1. Stages of the cell cycle.

FIG. 6.2. Expression pattern of cyclin proteins in liver of I. tridecemlineatus over the torpor-arousal cycle as determined by Western immunoblotting.

FIG. 6.3. Regulation pattern of cyclin proteins in skeletal muscle of $I$. tridecemlineatus as determined by Western immunoblotting.

FIG. 6.4. Transcriptional regulation of cyclin mRNA in liver of $I$. tridecemlineatus as determined by RT-PCR.

FIG. 6.5. Transcriptional regulation of cyclin mRNA in skeletal muscle of $I$. tridecemlineatus as determine by RT-PCR.

FIG. 6.6. Protein levels of CDKs in liver of I. tridecemlineatus as determined by Western immunoblotting.

FIG. 6.7. Protein levels of CDKs in skeletal muscle of I. tridecemlineatus as determined by Western immunoblotting.

FIG. 6.8. Phosphorylation of checkpoint kinases in liver of $I$. tridecemlineatus as determined by Western immunoblotting.

FIG. 6.9. Phosphorylation of checkpoint kinases in skeletal muscle of $I$. tridecemlineatus as determined by Western immunoblotting.

FIG. 6.10. Regulation pattern of cell cycle inhibitor proteins in liver of $I$. tridecemlineatus as determined by Western immunoblotting.

FIG. 6.11. Regulation pattern of cell cycle inhibitor proteins in skeletal muscle of I. tridecemlineatus as determined by Western immunoblotting. 
FIG. 6.12. Regulation pattern of PCNA protein and relative phosphorylation of p21CIP1 (Ser-146) in liver of I. tridecemlineatus as determined by Western immunoblotting.

FIG. 6.13. Regulation pattern of PCNA protein and relative phosphorylation of p21CIP1 (Ser-146) in skeletal muscle of I. tridecemlineatus as determined by Western immunoblotting.

FIG. 6.14. Partial cDNA sequence of cyclin D1

FIG 6.15. Cyclin D1 homology alignment.

FIG. 6.16. Partial cDNA sequence of cyclin E1

FIG 6.17. Cyclin E1 homology alignment.

FIG. 6.18. Partial cDNA sequence of cyclin $A 2$

FIG 6.19. Cyclin A 2 homology alignment.

FIG. 6.20. Partial cDNA sequence of cyclin B1

FIG 6.21. Cyclin B1 homology alignment.

FIG. 6.22. Mechanism of CKI inhibition of Cdks. 


\section{LIST OF TABLES}

Table 2.1. Antibody list showing primary antibodies used for Western blotting, their sources and dilution for use, as well as the molecular weight of the protein target.

Table 2.2. Custom primers sequences designed for target genes, indicated with optimal annealing temperature $(\mathrm{Tm})$ and PCR product size.

Table 2.3. Accession numbers of sequences retrieved from NCBI nucleotide collection used for homology alignment comparisons.

Table 4.1. Kinetic parameters of PTEN from skeletal muscle of euthermic and hibernating ground squirrels.

\section{LIST OF APPENDICES}

Appendix A List of publications

Appendix B Communications at scientific meetings

Appendix C PCR amplification cycles

Appendix D Malachite green standard curve

Appendix E RNA intergrity assessment (Pre-standardization of RNA concentration

Appendix F Comparison of end-point and real time PCR analysis of $\alpha$ Tubulin

Appendix G Comparison of GAPDH expression with Coomassie blue stain 
CHAPTER 1

\section{GENERAL INTRODUCTION}




\subsection{GENERAL INTRODUCTION}

Seasonal impacts on environmental habitat

The lifecycles of many organisms are dictated by their geographical location and their natural surroundings. Animals that inhabit temperate and polar regions of the earth experience major changes in environmental temperature over the year, and face serious challenges to survival from cold environmental temperatures and the limited food availability in the winter. As a result of this unfavorable environment, different species utilize different strategies to promote survival. Adaptations to the cold can take on many forms, and are categorized based on the need to survive at above $0^{\circ}$ or below $0^{\circ}$. Survival at temperatures below the freezing point of body fluids can be achieved by either freeze avoidance or freeze tolerance (Rickards et al., 1986). Goldenrod gall moth such as the Epiblema scudderiana undergoes freeze avoidance, whereby a derepression of colligative freezing point takes place. This is achieved through a large amount of glycerol production that functions as a cryoprotectant to sustain supercooling depression to as low as $-25^{\circ} \mathrm{C}$ (Rickards et al., 1986). Meanwhile amphibians such as Rana sylvatica undergoes freeze tolerance, a form of adaptation that results in ice formation within body fluids (Storey, 1992). Similar to freeze avoidance, cryopreservation is achieved with large production of cryoprotectants such as glucose, which defends against intracellular dessication (Storey and Storey, 1992). In conditions above the freeze point, animals can either migrate to a warmer climate, or maintain homoeothermic temperature by staying warm. Large populations of birds migrate during the wintering months of the year towards neotropical regions, while higher mammals such as human utilize shelters to stay warm. However, a 
subset of mammals undergoes hibernation, a form of dormancy that is characterized by an altered metabolism that is maintained while in a torpid state.

\section{Mammalian hibernation}

To date, mammalian hibernation has been observed in eight different groups of mammals: monotremes (echidna), rodents (ground squirrels and many others), marsupials (pygmy-possum), bats (little brown bat and many others), insectivores (hedgehog), carnivores (black bear), shrews (elephant shrew), and recently documented primates (selected lemurs) (Storey, 2010; Dausmann et al., 2004). For small hibernators such as bats, ground squirrels, and marmots hibernation involves prolonged periods of deep torpor, during which core body temperature $(\mathrm{Tb})$ falls to near ambient, interspersed with brief periods of arousal back to euthermia (FIG 1.1). Large hibernators such as black bears show a different pattern; although they still suppress metabolic rate during torpor, their large mass means that their bodies cool only to $30-36^{\circ} \mathrm{C}$, well above ambient temperature (Tøien et al., 2011).

The hibernation cycle is composed of a number of torpor-arousal bouts, as shown in FIG. 1.1. Depending on the species, torpor bouts can range between a few days to up to 4-5 weeks, and are interrupted by periods of interbout arousal between each cycle. In ground squirrels, the torpor-arousal cycle is characterized by six distinct phases that are determined based on the animal's physiological behaviours (such as $\mathrm{Tb}$ and respiration) (FIG 1.2). The preparation for hibernation begins in late summer, when animals undergo massive weight gain through hyperphagia, to increase their fat storage in white adipose tissues (Dark, 2005). As environmental temperatures begin to decline, hibernators 
undergo initial periods of test torpor cycles, where torpor bouts are short and shallow earlier in the season; this is followed by bouts of torpor that are progressively longer and deeper by midwinter (FIG. 1.2). The decline in body temperature as observed in the entrance phase of the torpor cycle is initiated by a rapid reduction in metabolic rate that takes precedence (FIG 1.3). This indicates that entry into a torpid state is actively regulated and not a passive process; ie. the decline in body temperature is the result, not the cause, of metabolic rate reduction. As previously demonstrated by Heldmaier et al. (2004), maximal reduction in metabolic rate is observed within $4-5$ hours into the torpor cycle, whereas maximal reduction in $\mathrm{Tb}$ is not achieved until $15-18$ hours (FIG. 1.3) (Heldmaier et al., 2004).

\section{Metabolic reprogramming}

Each bout of the torpor-arousal cycle is composed of six distinct stages, which are characterized based on the physiological status of the animal. Entry into torpor is initiated with rapid decrease in metabolic rate, leading to a reduction of $\mathrm{Tb}$ with a lowering of the hypothalamus set-point (Lowell and Splegelman, 2000). As Tb drops to near ambient (often $\sim 4-5^{\circ} \mathrm{C}$ when hibernating in a burrow), physiological actions such as heart rate are drastically reduced from 200 - 300 beats per min to as little as 5 beats per minute whereas respiration can decline to $4-6$ breath per min, from $100-200$ (McArthur and Milsom, 1991). Overall, basal metabolic rate can decrease by up to $95-99 \%$ during torpor (Geiser, 2004). To achieve such metabolic depression, specific regulatory mechanisms must take place to initiate and maintain metabolic reprogramming. Theoretically speaking, a global reduction in metabolism must be accomplished by 
altering the rate of ATP turnover and so, to maintain homeostasis, this means that the rates of both ATP consuming and ATP producing processes must be suppressed in concert. Indeed, a large number of previous studies have shown that major energy demanding processes such as protein synthesis, gene transcription, and ion channel pumps are all reversibly depressed during hibernation along with energy producing processes including glycolysis and mitochondrial function (Martin et al., 1999; Frerichs et al., 1999; Morin and Storey, 2006; MacDonald and Storey, 1999; Storey, 1997; van Breukelen and Martin, 2001; van Breukelen and Martin, 2002). In addition to reprioritization of energy usage, major shifts in fuel utilization also take place, with both alterations often considered to be hallmarks of metabolic reprogramming (Anderson and Weindruch, 2010). The significant increase in adipose content during the preparatory phases of hibernation and a respiratory quotient while torpid of 0.7 observed in ground squirrels indicates that lipid catabolism is the primary source of metabolic fuel during hibernation (South and House, 1967).

Regulatory mechanisms, reversible protein phosphorylation (RPP)

Frerichs et al. (1999) have previously demonstrated that protein synthesis in ground squirrel brain is strongly arrested for weeks during hibernation, in a manner that is independent of temperature effects (Frerichs et al., 1999). The observed reduction of protein synthesis by 75 -fold during hibernation could not simply be a product of $\mathrm{Q}_{10}$ effects, which could only account for a $\sim 13.6$-fold reduction (assuming a $\mathrm{Q}_{10}$ range of 2 3 for most enzymes) (Frerichs et al., 1999). This suggests that additional regulatory mechanisms must also be in place that suppress protein synthesis. Indeed, 
phosphorylation of eukaryotic initiation factor- $2 \alpha(\mathrm{eIF} 2-\alpha)$ was found to be greatly increased during hibernation, with a near 6.5-fold increase (Frerichs et al., 1999). The phosphorylation of eIF2- $\alpha$ at Ser-51 is known to form an eIF2-GDP structure that complexes with eIF2B, to inhibit the exchange of GDP to GTP that is required for the initiation of eukaryotic translational complex (Merrick, 1992).

The reversible addition of phosphate group catalyzed by the actions of protein kinases and protein phosphatases functions as a rapid means of post-translational modification, which can influence the activity state of many functional proteins (Storey, 2010). The coordinated and readily reversible nature of RPP is a central mechanism that has previously been shown to regulate a wide range of cellular processes during hibernation. Indeed, the shift in fuel utilization from carbohydrate to lipid metabolism in hibernators also depends on RPP, specifically the phosphorylation of pyruvate dehydrogenase by pyruvate dehydrogenase kinase isoform 4 , which reduces the rate of glucose oxidation by inhibiting the conversion of pyruvate to acetyl-CoA (Rowles et al., 1996). Indeed, the levels of dephosphorylated pyruvate dehydrogenase (active form) were reduced from $60-80 \%$ in euthermia to less than $5 \%$ in hibernation (Brooks and Storey, 1992). The actions of RPP have also previously been linked to regulation of $\mathrm{Na}^{+} \mathrm{K}^{+}-$ ATPase pump activities during hibernation. The $\mathrm{Na}^{+} \mathrm{K}^{+}-\mathrm{ATPase}$ pump in the plasma membrane, utilizes ATP to move ions against their concentration gradients, maintaining low $\mathrm{Na}^{+}$and high $\mathrm{K}^{+}$intracellular concentrations. Although essential for life, it is one of the most energy consuming processes in endotherms, utilizing 5-40\% of cellular energy depending on the tissue (Clausen, 1986). MacDonald and Storey (1999) showed that stimulation with protein kinase resulted in a decrease of $\mathrm{Na}^{+} \mathrm{K}^{+}$-ATPase activity, which 
was reversed upon dephosphorylation via phosphatases (MacDonald and Storey, 1999). This demonstrated that the suppression of $\mathrm{Na}^{+} \mathrm{K}^{+}$-ATPase activity during torpor is a product of protein phosphorylation, which can be readily reversed during arousal by protein phosphatases.

Other examples of differential phosphorylation have also been demonstrated in the regulation of other major cellular processes, namely mRNA transcription and antioxidant defense. Gene transcription accounts for $\sim 1-10 \%$ of total energy usage in the cell, and functions as the cellular mechanism that regulates levels of mRNA transcripts (Rolfe and Brown, 1997). Studies by Morin and Storey (2006) have shown that phosphorylation in components of the transcriptional machinery RNA polymerase II is significantly increased during hibernation; as well, histone H3 phosphorylation was reduced during hibernation. Increased RNA polymerase II phosphorylation at Ser-5 has previously been linked to transcriptional control, while increased H3 phosphorylation at Ser-10 has been associated with transcriptional activation (Kim et al., 1997; Cheung et al., 2000). Both of the observed modification of the transcriptional machinery are consistent with a reduced rate of RNA polymerase II activity, suggesting an overall reduced state of transcriptional activation during torpor.

\section{Animal model}

Ground squirrels are among the most commonly used model animals for studying hibernation and in North America, several species of the Ictidomys genus hibernate, including the 13-lined ground squirrel, Ictidomys tridecemlineatus, which is the model used in this study. I. tridecemlineatus is found across most of the grasslands of central 
North America with an annual life cycle that includes a long period of hibernation that averages about 240 days (Linzey \& Hammerson, 2008; McCarly, 1996). Typical body size of an adult is about $30 \mathrm{~cm}$ in length and 200-400 grams in weight during the active season. However, in late summer, ground squirrels undergo hyperphagia and greatly increase their body weight to nearly 750 grams, laying down huge reserves of body fat (Linzey \& Hammerson, 2008). The ground squirrel is an omnivore, feeding on grasses, seeds, small insects, and fruit as its primary source of nutrient. As the availability of food declines along with temperature, I. tridecemlineatus typically enter hibernation during October, and emerge in early spring (March to April) during the breeding periods (Linzey \& Hammerson, 2008). In this thesis, the primary focus is on metabolic regulation in the liver and skeletal muscle over torpor-arousal cycles; in addition, some aspects of cardiac muscle metabolism are also examined in Chapter 3.

\section{Cardiac Muscle}

The heart functions to provide and replenish oxygenated blood throughout the body via the cardiovascular system, a physiological function that has previously been shown to be greatly suppressed during hibernation (Zatzman, 1984). Although heart beat is reduced by nearly 100-fold, evidence of muscular hypertrophy has been found during torpor, and the increased strength of contraction appears to be needed to compensate for reduced heart rate and increased blood viscosity at cold body temperatures (Wicker et al., 1991; Storey and Storey, 2004). A number of studies have linked physiological changes in cardiac muscle to alterations at the molecular level. Expression of multiple genes involved in major regulatory processes have been found to be upregulated during torpor, 
including myocyte enhancing factor-2 (MEF-2), glucose transporter-4 (GLUT-4), pyruvate dehydrogenase kinase 4 (PDK4), fatty acid binding protein, peroxisome proliferator-activated receptor (PPAR- $\gamma$ ), myosin light chain 1, and mitochondrial ND2 (Tessier and Storey, 2012; Buck et al., 2002; Hittel and Storey 2001; Eddy et al., 2005; Fahlman et al., 2000). The increased responsive of these genes provides evidence of enhanced fatty acid metabolism along with increased cardiomyocte functions during torpor. Despite an overall reduction in metabolic functions during torpor in ground squirrels, a recent study has shown that little to no change in mitochondrial metabolism in cardiac muscle during torpor. Torpor induction had no effect on the rate of mitochondrial oxidation of a variety of substrates in cardiac muscle such as pyruvate, palmitoyl carnitine, $\beta$-hydroxybutyrate, succinate, or glutamate (Gallerger and Staples, 2013). The low contribution of cardiac muscle to overall metabolic rate $(<1 \%$ in rodents $)$ could suggest preferential retention of molecular processes during torpor, whereby functions of a critical tissue such as heart are maintained albeit at a reduced rate.

\section{Liver}

Liver functions as a central organ involved in the coordination and regulation in a wide range of functions that affect the whole body; these include detoxification reactions, protein synthesis, glycogen storage, and hormone production. Previous studies have shown decreased glycogen phosphorylase activity along with reduced concentrations of fructose-2,6-bisphosphate during hibernation (Brooks and Storey, 1992). Decreased glycolytic flux is observed along with the overexpression of proteins involved in fatty acid metabolism in the liver during torpor; this is consistent with the switch towards lipid 
as the primary source of metabolic fuel during torpor (Shao et al., 2010). A number of proteins involved in detoxification and tissue protection were also underexpressed during torpor, these including aldehyde oxidase, superoxide dismutase, cytochrome P450 family proteins (Shao et al., 2010). As well, mitochondrial oxidative capacities have been shown to be severely suppressed during torpor (Brown et al., 2012). Although evidence suggests that selected processes of the liver are likely to be reduced during torpor, liver also provides many functions that are key to survival during torpor. The reliance on lipid metabolic fuel requires function of many lipolytic enzymes involved in lipid transport, such as apolipoprotein A1 that is synthesized in the liver. Indeed, expression of many lipid metabolic proteins such as liver isoforms of fatty acid binding protein, acyl CoA dehydrogenase, and carboxylesterase 2 are all found to be upregulated during torpor (Epperson et al., 2004). The hyperactivation or reduction in the expression of many protein markers in liver suggest that selected functions of liver are maintained and are critical for survival during torpor. Meanwhile non-essential liver functions are reduced, along with the observation of an overall reduction in metabolic rate during torpor.

\section{Skeletal muscle}

Skeletal muscle contributes to the movement and mobility of the animal, a function that is restricted during torpor. Although mainly inactive during torpor, skeletal muscles play a vital role in metabolic restoration during the arousal phase via heat generation from shivering. The initial phase of warming the body is conducted by brown adipose but once $\mathrm{Tb}$ is above $\sim 15^{\circ} \mathrm{C}$, muscle shivering is initiated and contributes to overall heat generation. Prolonged disuse of muscle during torpor leads to some atrophy 
but, overall, the extent of atrophy is much less than compared with non-hibernating species, suggesting possible protective mechanisms against muscle wasting during torpor (Wicker et al., 1991). At the molecular level, a number of studies have documented regulation of muscle metabolism during torpor. Mitochondrial metabolic suppression is also observed in skeletal muscle; for example, reduced rates of succinate-fueled mitochondrial respiration were seen during torpor (Brown et al., 2012). Hexokinase, a rate-limiting enzyme in glycolysis, showed a 33\% reduction in activity during hibernation whereas creatine kinase showed a $20 \%$ reduction in activity along with decreased expression of both mRNA and protein during hibernation (Abnous and Storey, 2007; Abnous and Storey, 2008). Activity of MAP-activated protein kinase 2 was also reduced by $63 \%$ during torpor, suggesting lower mitogen signaling (Abnous et al., 2012). The overall pattern of suppression seen in a number of different metabolic pathways suggest that skeletal muscle function is strongly suppressed during torpor, and may contribute significantly to total body energy savings.

\subsection{OBJECTIVES AND HYPOTHESES}

Metabolic depression is commonly observed in vertebrates and non-vertebrates when faced with different environmental stresses, including low oxygen, high or low temperature, freezing, desiccation, etc. (Zhao and Haddad, 2011; Fielenbach and Antebi, 2008; McNally et al., 2002; Storey and Storey, 2007). This type of metabolic reprogramming is also conserved in many species of mammals, however, transferrable knowledge towards human applications continues to be a void. In hibernators, each tissue 
displays a specific mode of response during entry into torpor, which is dictated by the physiological significance and/or role the tissue to the whole organism. The importance of understanding the molecular nature of mammalian hibernation provides not only a comparative view to elucidate the principles of biochemical adaptation, but potential valuable applications toward medical sciences, such as the improvement of hypothermic storage techniques for use in organ transplantation. Therefore, I have chosen to take a molecular based approach to characterize expression and functionality of key signal transduction pathways over the torpor-arousal cycle in I. tridecemlineatus, with a focus on antagonistic pro-growth and anti-growth signaling pathways.

\section{Objective 1: Signaling mechanisms in translational arrest}

Energy use for protein synthesis can account for $20-30 \%$ of standard metabolic rate and it is not surprising, therefore, that synthesis of new protein products is severely restricted in ground squirrel organs during torpor (Frerichs et al., 1999; Rolfe and Brown, 1997). Components of the translational complex are regulated via the mammalian target of rapamyacin (mTOR) pathway, a signaling pathway that integrates inputs from several protein kinase signaling cascades that are themselves responsive to different inputs (e.g. amino acid or energy availability, growth factors, insulin, etc.) The mTORC1 complex plays a major role in regulating cellular growth by promoting protein synthesis via the activation of p70S6 kinase (p70S6K) and inhibition of the eukaryotic translation initiation factor 4E-binding protein 1 (4E-BP1). Through downstream targets of $\mathrm{p} 70 \mathrm{~S} 6 \mathrm{~K}$ and 4E-BP1, mTORC1 regulates the rate of mRNA binding and ribosomal assembly, which are considered to be rate-limiting process in translational initiation (Matthews et 
al., 2000). The regulation of mTORC1 is of particular interest because of its ability to control energy consumption based on nutrient availability, which is especially crucial during the non-feeding hibernation season when availability of amino acids for new protein synthesis is limited.

\section{Hypothesis 1: The positive regulatory role of mTOR signaling in} promoting protein synthesis is repressed during the torpor stages of hibernation, as a mechanism to reduce energy expenditure. Tissue specific modes of regulation will reflect physiological roles of cardiac and skeletal muscle during torpor.

Chapter 3 tests this hypothesis by examining the regulation of the mTOR signaling pathway in cardiac and skeletal muscle over the torpor-arousal cycle. Phosphorylation of key regulatory kinases is examined, along with upstream and downstream targets of the mTORC1 complex.

\section{Objective 2: Regulation of PTEN phosphatase}

PTEN is a protein phosphatase that acts as a tumour suppressor and is known to negatively regulate the insulin signaling pathway. Through its phosphatase activity towards the lipid second messenger $\mathrm{PIP}_{3}$ [phosphatidylinositol (3,4,5)-trisphosphate], PTEN acts as an upstream negative regulator of the Akt / mTOR signaling pathway, by catalyzing the dephosphorylation of $\mathrm{PIP}_{3}$ to $\mathrm{PIP}_{2}$. The levels of $\mathrm{PIP}_{3}$ are known to influence the activation of phosphoinositide-dependent kinase-1 (PDK-1), an upstream regulator of Akt. Dysfunctional PTEN is crucial in promoting growth and proliferation in many cancer malignancies, and acts as a major moderator in cellular growth. This 
indicates that PTEN is an interestingly target in the regulation of metabolic events during hibernation, especially with evidence of reduced energy levels during torpor.

Hypothesis 2: Increased activity of PTEN functions as an upstream regulatory mechanism to repress the mTOR signaling pathway during torpor stages of the hibernation cycle, when energy levels are low.

Chapter 4 tests this hypothesis through examining the enzymatic regulation of PTEN phosphatase during torpor; phosphatase activity and PTEN structural stability is also measured in skeletal muscle. Regulation of PTEN and downstream PDK-1 are analyzed at transcriptional and translational levels over the torpor arousal cycle.

\section{Objective 3: Activation of anti-proliferatory signaling during}

\section{torpor}

The transforming growth factor beta (TGF- $\beta$ ) signalling pathway involves the activation of a ligand-based receptor by a variety of cytokines such as TGF- $\beta$, Nodals, and activins, leading to the activation of the Smad family of transcription factors, and resulting in changes in expression of downstream gene targets. Considered as antiproliferatory factors, Smad 2 and Smad 3 activate the expression of p21CIP1 and $\mathrm{p} 15 \mathrm{INK} 4 \mathrm{~b}$ along with a number of microRNAs involved in metabolic regulation such as miR-21, miR-29b, and miR-155. Cell cycle inhibitors such as p21CIP1 and p15INK4b function to promote cell cycle arrest in early stages of cell division. Meanwhile miR-21 and miR-29b have been shown to negatively regulate the insulin signaling pathway. The 
regulatory mechanisms of the Smad family of proteins are of interest during hibernation, due to their anti-proliferatory nature, functioning to repress cell growth.

Hypothesis 3: Activation of Smad 2 and Smad 3 during torpor function to promote a global reduction in energy expenditure. Via activation of downstream genes, Smad serves to suppress pro-growth processes such as cell division during torpor.

Chapter 5 tests this hypothesis by examining the gene and protein expression of two Smad transcription factors during torpor in liver and skeletal muscle. Regulation of these Smads is evaluated at the transcriptional and translational level along with DNAbinding activity, and subcellular localization. In addition, transcriptional profiling of Smad downstream targets is analyzed.

\section{Objective 4: Mechanism of cell cycle arrest in torpor.}

The cell cycle is composed of four distinct stages (G1, S, G2, M) with each stage regulated by different groups of cyclins and cyclin dependent kinases (Cdk). Similar to protein synthesis, cell division is another energy expensive cellular process, and cell cycle arrest has previously been shown to be integral to hypometabolism in systems such as the dauer formation in $C$. elegans. Since each stage of the cell cycle is regulated by a different combination of cyclins and Cdks, the state of the cell cycle progression can be assessed based on the relative expression levels of these proteins. Transition to each stage of the cell cycle also requires specific check point regulators such as check point kinase 1, and 2 (Chk1, 2). The checkpoint kinases activate members of the Cdc25 family of 
phosphatases, which subsequently activate the cyclin: CDK complexes required for progression between stages of the cell cycle. However, in the event of DNA damage (a potential event during long term torpor), check point kinases are activated, resulting in cell cycle arrest. Each of the four phases of the cell cycle is also regulated by a family of cell cycle inhibitors (CKI) which inhibit cell cycle progression by inhibiting the functions of cyclins, or cyclin-Cdk complexes.

Hypothesis 4: Mechanisms of cell cycle arrest will be evident in liver (proliferative), but not muscle (non-proliferative) tissues over the torpid stages of hibernation. Arrest will be marked by either downregulation of positive cell cycle regulators such as cyclins and Cdks, or activation of negative cell cycle regulators such as CKIs.

Chapter 6 tests this hypothesis by examining the gene and protein expression of major cell cycle components in all four phases of cell division. Analysis of liver and skeletal muscle compares and contrasts regulatory mechanisms in proliferative (liver) vs. terminally differentiated (muscle) tissues. 


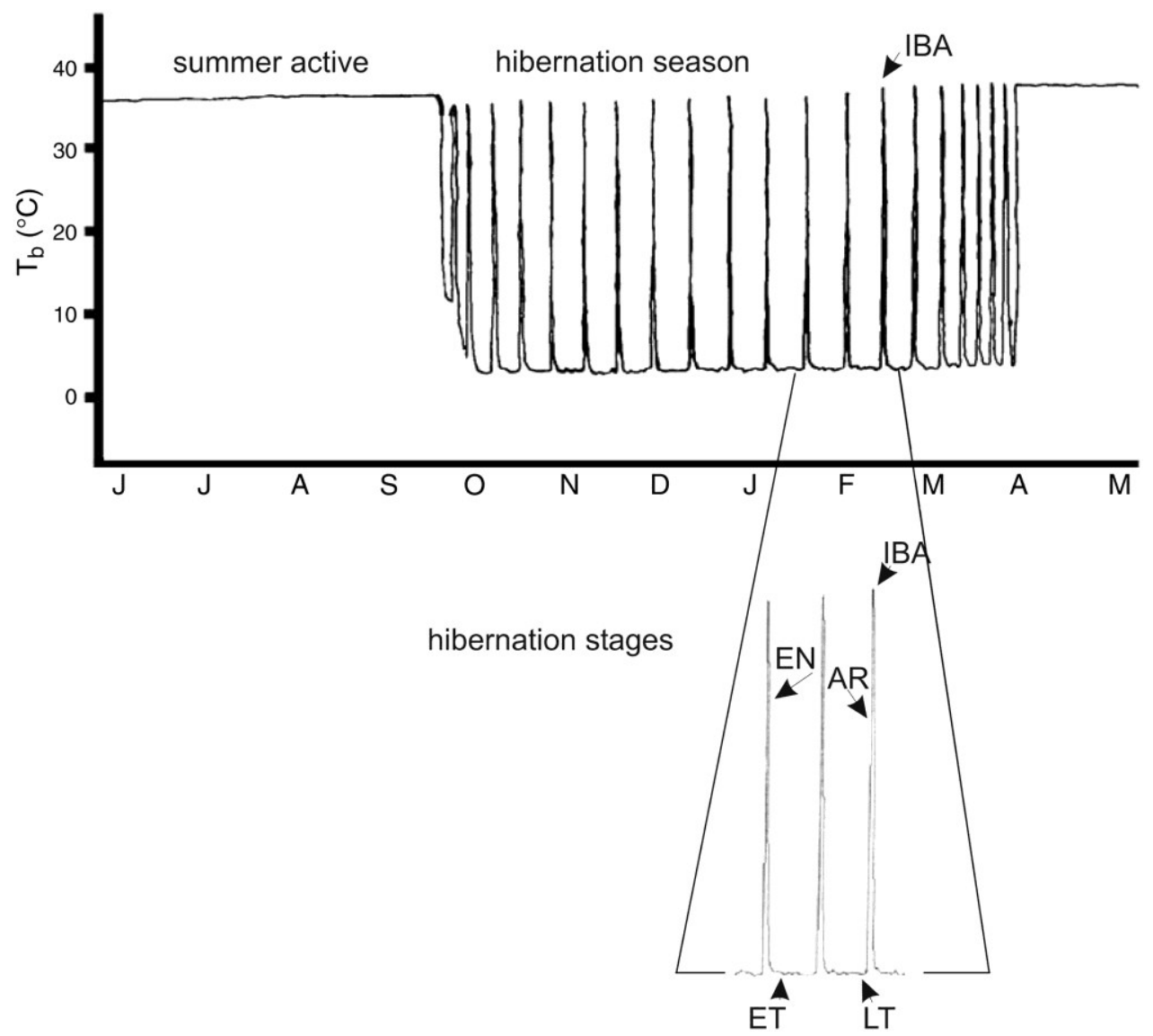

FIG. 1.1. Pattern of changes in body temperature of a ground squirrel over a one year period showing multiple bouts of torpor over the winter hibernation season.

The hibernation season was initiated in mid-September and lasted to the beginning of April. Hibernation consists of multiple torpor bouts, each cycle is interrupted by a full arousal back to euthermia before re-entry into torpor. Insert shows an expanded view over three torpor bouts (From Carey et al., 2003). 


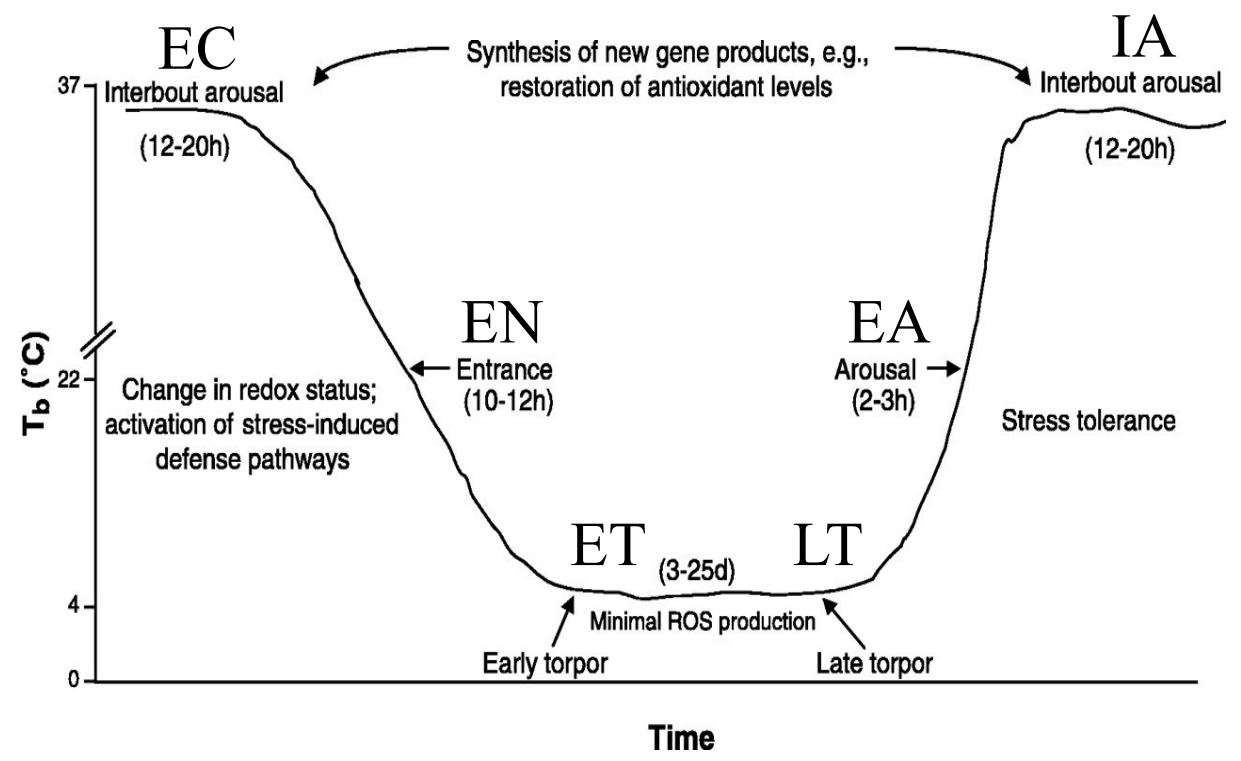

FIG. 1.2. Stages of the torpor-arousal cycle.

Each torpor-arousal cycle can last between a few days to $4-5$ weeks, and is characterized by distinct stages determined based on animal body temperature and respiratory rate. The parameters of these stages as sampled for experiments undertake in this thesis are: (1) Euthermic control in the cold room (EC; also known as interbout arousal) - squirrels had a stable $\mathrm{Tb}\left(34-37^{\circ} \mathrm{C}\right)$ in the $5^{\circ} \mathrm{C}$ cold room, (2) entrance into torpor $(\mathrm{EN})-\mathrm{Tb}$ falling with sampling between $31^{\circ}$ and $18^{\circ} \mathrm{C},(3)$ early torpor $(\mathrm{ET})-\mathrm{Tb}$ had been $\sim 5-8^{\circ} \mathrm{C}$ for $\sim 24 \mathrm{~h}$, (4) long term / late torpor (LT) $-\mathrm{Tb}$ constant at $\sim 5-8^{\circ} \mathrm{C}$ for $>5$ days, (5) early arousal $-\mathrm{Tb}$ rising to $\sim 12^{\circ} \mathrm{C}$ with a rapid increase in respiration $\geq 60$ breaths/min, and (6) full interbout arousal (IA) - Tb returned to $\sim 34-37^{\circ} \mathrm{C}$ for at least 18 h after at least 3 days of continuous torpor (modified from Carey et al., 2003). 


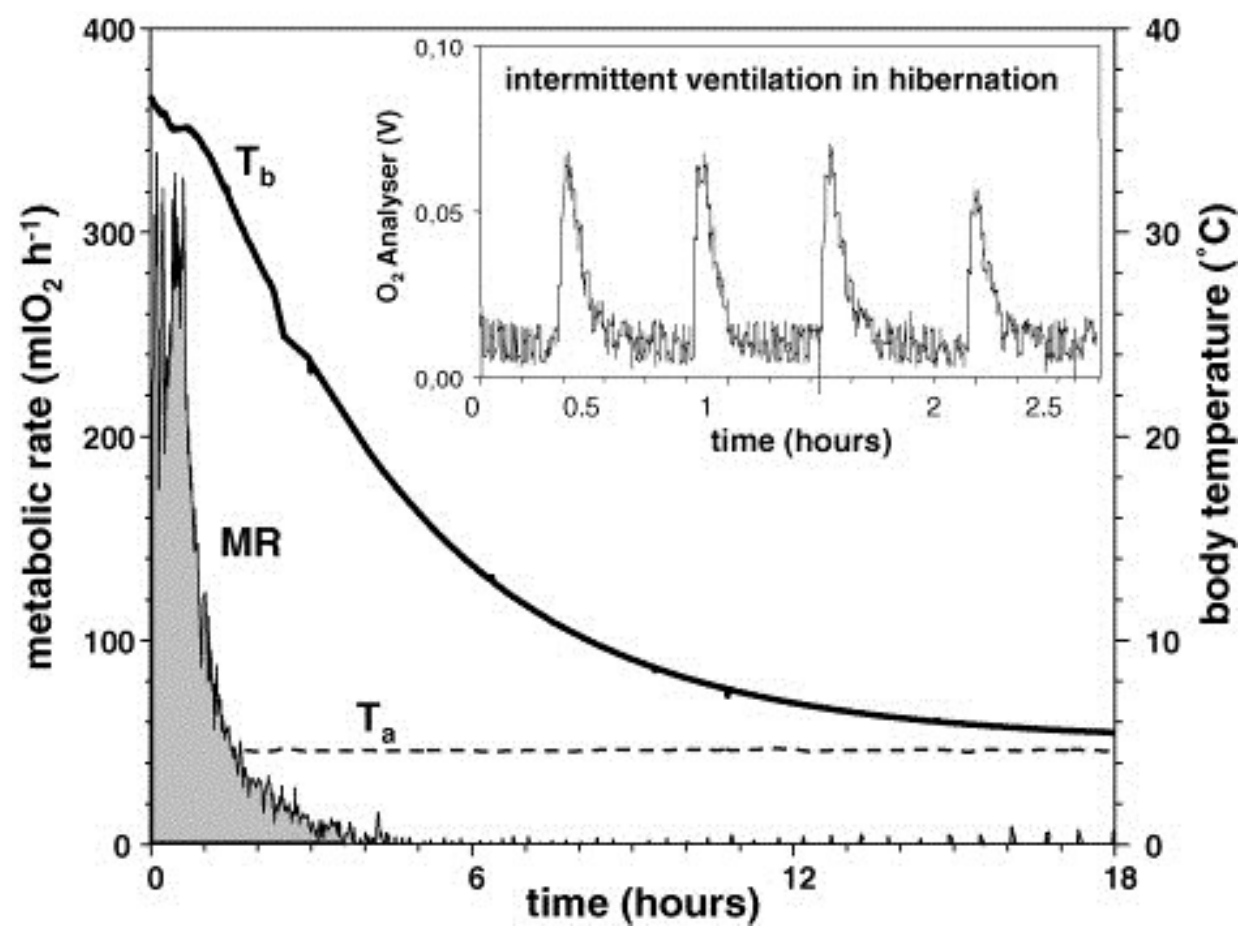

FIG 1.3. Body temperature and metabolic rate during entry into torpor of Alpine marmot (Marmota marmota).

The reduction of body temperature observed during torpor is a result of the prior suppression of metabolic rate that takes precedence. In the torpor bout shown here, the maximum reduction of metabolic rate was observed within the first $4-5$ hours during the entry phase into torpid, whereas the lowest $\mathrm{Tb}$ was not observed until $16-18$ hours. This suggests that entry into hibernation is not a passive effect of temperature reduction, but rather an active repression of metabolism (From Heldmaier et al., 2004). 


\section{CHAPTER 2}

MATERIALS \& METHODS 


\subsection{Animal treatments}

Animal studies were conducted as previously described by McMullen and Hallenbeck (2010). Briefly, thirteen-lined ground squirrels (Ictidomys tridecemlineatus), weighing 150-300 g, were wild-captured and supplied by TLS Research, Bloomingdale, IL. Animals were transported to the Animal Hibernation Facility, National Institute of Neurological Disorders and Stroke, NIH, Bethesda, MD where all experiments were conducted by the laboratory of Dr. J.M. Hallenbeck. Animals were individually housed in shoebox cages in a holding room with an ambient temperature of $21^{\circ} \mathrm{C}$ under a $12 \mathrm{~h}$ light:12h dark cycle. Animals were fitted with a sensor chip (IPTT-300; Bio Medic Data Systems) injected subcutaneously while the squirrels were anesthetized with 5\% isofluorane. Animals were fed water and standard rodent chow ad libitum until they gained sufficient lipid stores to enter hibernation. To enable a natural transition into torpor, animals were transferred to constant darkness in an environmental chamber at 4$5^{\circ} \mathrm{C}$. Body temperature $(\mathrm{Tb})$, time and respiration rate were monitored and used to determine the stage of torpor-arousal cycle. All animals had been through several torporarousal bouts prior to sampling at a set stage. Animals were euthanized and samples of hindlimb thigh muscles, whole heart, and liver were collected at six different time points defined by $\mathrm{Tb}$, duration of torpor, and respiration rate: (1) Euthermic in the cold room (EC) - euthermic squirrels had a stable $\mathrm{Tb}\left(34-37^{\circ} \mathrm{C}\right)$ for at least three days. $\mathrm{EC}$ animals were capable of entering torpor but had not done so in the past 72 hours. (2) early entrance into torpor (EN) (Tb falling with sampling between $31^{\circ}$ and $18^{\circ} \mathrm{C}$ ), (3) early torpor phase (ET) ( $\mathrm{Tb}$ of $\sim 5-8^{\circ} \mathrm{C}$ for $<24 \mathrm{~h}$ ), (4) late torpor phase (LT) (Tb of $\sim 5-8^{\circ} \mathrm{C}$ for $>5$ days), (5) early arousal (EA) ( $\mathrm{Tb}$ rising to $\sim 12^{\circ} \mathrm{C}$ with a rapid increase in respiration 
$\geq 60$ breaths/min, (6) interbout arousal (IA) (Tb returned to $\sim 34-37^{\circ} \mathrm{C}$ for at least $18 \mathrm{~h}$ after at least 3 days of torpor). Animals were sampled as in described McMullen and Hallenbeck (2010) and tissues were shipped to Carleton University on dry ice and stored at $-80^{\circ} \mathrm{C}$ until use.

\subsection{Total protein isolation and Western blots}

Frozen tissue samples $(\sim 0.5 \mathrm{~g})$ were homogenized 1:2 w:v in buffer containing 20 mM HEPES, $200 \mathrm{mM} \mathrm{NaCl}, 0.1 \mathrm{mM}$ EDTA, $10 \mathrm{mM} \mathrm{NaF}, 1 \mathrm{mM} \mathrm{Na}_{3} \mathrm{VO}_{4}$, and $10 \mathrm{mM}$ ßglycerol-phosphate, adjusted to $\mathrm{pH} 7.5$, supplemented with $1 \mu \mathrm{L}$ of protease inhibitor cocktail (BioShop \#PIC001) and a few crystals of phenylmethylsulphonyl fluoride added immediately before homogenization. Samples were homogenized on ice for $\sim 15 \mathrm{sec}$ with a Polytron PT1000 homogenizer and centrifuged at $4^{\circ} \mathrm{C}$ for $15 \mathrm{~min}$ at $10,000 \mathrm{x} g$. Supernatants were collected and soluble protein concentrations were determined by the Coomassie blue dye method using the Bio-Rad prepared reagent and bovine serum albumin as the standard. Samples were adjusted to the same concentration with the addition of small aliquots of homogenizing buffer and then measured aliquots were mixed 1:1 v:v with 2X SDS sample buffer [100 mM Tris-base, 4\% w/v sodium lauryl sulphate (SDS), 20\% v:v glycerol, 0.2\% w:v bromophenol blue, 10\% v:v 2mercaptoethanol] and boiled for $5 \mathrm{~min}$. A $30 \mu \mathrm{g}$ aliquot of protein was loaded into each well and run on SDS-PAGE gels (6\%-15\% depending on the protein being separated) for 60-120 min at $180 \mathrm{~V}$. Proteins were then wet transferred onto polyvinylidene fluoride (PVDF) membranes in transfer buffer solution (25 mM Tris pH 8.5, $192 \mathrm{mM}$ glycine, 10\% v:v methanol) for $90 \mathrm{~min}$ at $320 \mathrm{~mA}$. Following the transfer, membranes were 
blocked with 5\% milk dissolved in Tris-buffered saline with Tween-20 (TBST: $50 \mathrm{mM}$ Tris-HCl, $150 \mathrm{mM} \mathrm{NaCl}, 0.05 \% \mathrm{v}: \mathrm{v}$ Tween 20, pH 6.8) for $15 \mathrm{~min}$. Membranes were then probed with primary antibodies $\left(1: 1000 \mathrm{v}: \mathrm{v}\right.$ dilution in TBST) overnight at $5^{\circ} \mathrm{C}$. Subsequently, membranes were incubated with HRP-linked anti-rabbit IgG secondary antibody (1:4000 v:v in TBST) for $1 \mathrm{~h}$, and immunoblots were developed using enhanced chemiluminescence $(\mathrm{ECL})$ reagents. Western blot band densities were analyzed using a ChemiGenius Bio-Imaging System with GeneTools software (SynGene, Frederick, MD). Immunoblot bands in each lane were standardized against a group of Coomassie stained proteins that did not show any variation between experimental states and were not near the molecular weight of the protein of interest. This method was validated by immunoblotting of a housekeeping gene (GAPDH; glyceraldehyde-3-phosphate dehydrogenase) that showed stable expression in skeletal muscle, heart, and liver samples over all experimental states and also stable expression when expressed relative to the combined density of the group of Coomassie stained protein bands.

\subsection{Subcellular protein isolations}

Separation of cytoplasmic and nuclear fractions was performed with $0.5 \mathrm{~g}$ tissue samples, homogenized with $1 \mathrm{~mL}$ of homogenization buffer (10 mM HEPES, $10 \mathrm{mM}$ $\mathrm{KCl}, 10 \mathrm{mM}$ EDTA, 1 mM DTT, pH 7.9) using 20 gentle strokes using a Dounce homogenizer. Prior to homogenization, $10 \mu \mathrm{L}$ of Protease inhibitor Cocktail (Sigma) was added to the samples. Homogenized samples were centrifuged at $10,000 \times \mathrm{g}$ for $10 \mathrm{~min}$ at $4^{\circ} \mathrm{C}$ and the supernatant (containing the cytoplasmic fraction) was removed. The pellet was then incubated with $200 \mu \mathrm{L}$ nuclear extraction buffer (20 mM HEPES, pH 7.9, 400 
$\mathrm{mM} \mathrm{NaCl}, 1 \mathrm{mM}$ EDTA, 10\% v/v glycerol, $1 \mathrm{mM}$ DTT) on ice, rocking for $1 \mathrm{~h}$ with occasionally stirring. Samples were then centrifuged at $10,000 \mathrm{x} g$ for $15 \mathrm{~min}$ at $4^{\circ} \mathrm{C}$. Supernatant (containing the nuclear fraction) was then removed, soluble protein concentration was measured and then samples were standardized to a constant concentration. Samples used for DNA binding assays were stored as is at $-20^{\circ} \mathrm{C}$, while samples used for Western blotting were created as described above. Integrity of nuclear extracts was confirmed by Western blotting using histone $\mathrm{H} 3$ antibody.

Table 2.1. Antibody list showing primary antibodies used for Western blotting, their sources and dilution for use, as well as the molecular weight of the protein target.

\begin{tabular}{|c|c|c|c|}
\hline Antibodies & $\begin{array}{c}\text { Molecular } \\
\text { Weight } \\
\sim \text { kDa }\end{array}$ & Company & Dilution \\
\hline \multicolumn{3}{|l|}{ Chapter 3} & \\
\hline GAPDH & $37 \mathrm{kDa}$ & $\begin{array}{l}\text { Santa Cruz } \\
\text { Biotechnology, SC- } \\
25778\end{array}$ & $1: 1000$ \\
\hline Phospho-Akt (Ser-473) & $60 \mathrm{kDa}$ & GenScript, A00272 & $1: 1000$ \\
\hline Phospho-TSC2 (Thr-1462) & $200 \mathrm{kDa}$ & Abgent, AP3471A & $1: 500$ \\
\hline mTOR & $289 \mathrm{kDa}$ & Cell Signaling, 2972 & $1: 1000$ \\
\hline Phospho-mTOR (Ser-2448) & $289 \mathrm{kDa}$ & GenScript, A00643 & $1: 1000$ \\
\hline $\begin{array}{l}\text { Phospho-S6 ribosomal protein } \\
\text { (Ser-235) }\end{array}$ & $32 \mathrm{kDa}$ & GenScript, A00315 & $1: 1000$ \\
\hline Phospho-P70S6K (Thr-389) & $70 \mathrm{kDa}$ & $\begin{array}{l}\text { Santa Cruz } \\
\text { Biotechnology, SC- } \\
11759\end{array}$ & $1: 1000$ \\
\hline GßL (mLST8) & $38 \mathrm{kDa}$ & Cell Signaling, 3274S & $1: 1000$ \\
\hline Phospho-PRAS40 (Thr-246) & $40 \mathrm{kDa}$ & $\begin{array}{l}\text { Santa Cruz } \\
\text { Biotechnology, SC- } \\
32629\end{array}$ & $1: 1000$ \\
\hline Phospho-4E-BP (Thr-36) & $15 \mathrm{kDa}$ & GenScript, A00329 & $1: 1000$ \\
\hline
\end{tabular}




\begin{tabular}{|c|c|c|c|}
\hline \multicolumn{3}{|l|}{ Chapter 4} & \multirow[b]{2}{*}{$1: 1000$} \\
\hline PTEN & $54 \mathrm{kDa}$ & Cell Signaling, 9188 & \\
\hline $\begin{array}{l}\text { Phospho-PTEN (Ser-380/Thr- } \\
\text { 382/383) }\end{array}$ & $54 \mathrm{kDa}$ & Cell Signaling, 9549 & $1: 1000$ \\
\hline $\begin{array}{l}\text { Non-phospho PTEN (Ser- } \\
\text { 380/Thr-382/Thr-383) }\end{array}$ & $54 \mathrm{kDa}$ & Cell Signaling, 7960 & $1: 1000$ \\
\hline PDK1 & $60 \mathrm{kDa}$ & Cell Signaling, 3062 & $1: 1000$ \\
\hline Phospho-PDK1 (Ser-241) & $60 \mathrm{kDa}$ & Cell Signaling, 3438 & $1: 1000$ \\
\hline \multicolumn{4}{|l|}{ Chapter 5} \\
\hline Smad2 & $60 \mathrm{kDa}$ & Cell Signaling, 5339 & $1: 1000$ \\
\hline Smad3 & $52 \mathrm{kDa}$ & Cell Signaling, 9523 & $1: 1000$ \\
\hline Smad4 & $70 \mathrm{kDa}$ & Cell Signaling, 9515 & $1: 1000$ \\
\hline Phospho-Smad2 (Ser-465/467) & $60 \mathrm{kDa}$ & Cell Signaling, 3108 & $1: 1000$ \\
\hline Phospho-Smad3 (Ser-423/425) & $52 \mathrm{kDa}$ & Cell Signaling, 9520 & $1: 1000$ \\
\hline Smurf 2 & $90 \mathrm{kDa}$ & GenScript, A01654-40 & $1: 1000$ \\
\hline \multicolumn{4}{|l|}{ Chapter 6} \\
\hline cyclin D1 & $36 \mathrm{kDa}$ & $\begin{array}{l}\text { Santa Cruz } \\
\text { Biotechnology, SC-717 }\end{array}$ & $1: 1000$ \\
\hline cyclin E1 & $56 \mathrm{kDa}$ & Abcam, kit-ab6552 & $1: 1000$ \\
\hline cyclin A2 & $55 \mathrm{kDa}$ & Abcam, kit-ab6552 & $1: 1000$ \\
\hline cyclin B1 & $58 \mathrm{kDa}$ & Abcam, kit-ab6552 & $1: 1000$ \\
\hline CDK 2 & $33 \mathrm{kDa}$ & $\begin{array}{l}\text { Santa Cruz } \\
\text { Biotechnologies, SC-748 }\end{array}$ & $1: 1000$ \\
\hline CDK 4 & $30 \mathrm{kDa}$ & Abcam, kit-ab6553 & $1: 1000$ \\
\hline CDK 6 & $36 \mathrm{kDa}$ & Abcam, kit-ab6553 & $1: 1000$ \\
\hline CDC 2 (CDK 1) & $33 \mathrm{kDa}$ & GenScript, A01295 & $1: 1000$ \\
\hline p-CDK (Thr-14/ Tyr-15) & $34 \mathrm{kDa}$ & $\begin{array}{l}\text { Santa Cruz } \\
\text { Biotechnologies, SC- } \\
\text { 28435-R }\end{array}$ & $1: 1000$ \\
\hline $\mathrm{p}-15 / \mathrm{p} 16$ & $15,16 \mathrm{kDa}$ & $\begin{array}{l}\text { Santa Cruz } \\
\text { Biotechnologies, SC- } \\
28260\end{array}$ & $1: 1000$ \\
\hline $\mathrm{p} 21$ & $21 \mathrm{kDa}$ & $\begin{array}{l}\text { Santa Cruz } \\
\text { Biotechnologies, SC-397 }\end{array}$ & $1: 1000$ \\
\hline Phospho-p21 (Ser-146) & $21 \mathrm{kDa}$ & $\begin{array}{l}\text { Santa Cruz } \\
\text { Biotechnologies, SC- } \\
12902\end{array}$ & $1: 1000$ \\
\hline
\end{tabular}




\begin{tabular}{|l|c|l|c|}
\hline PCNA & $29 \mathrm{kDa}$ & Abcam, ab15497 & $1: 1000$ \\
\hline Cdc25C (Ser-216) & $60 \mathrm{kDa}$ & GenScript, A00420 & $1: 1000$ \\
\hline Cdc25A (Ser-76) & $70 \mathrm{kDa}$ & GenScript, A00439 & $1: 1000$ \\
\hline Chk 1(Ser-296) & $56 \mathrm{kDa}$ & GenScript, A00727 & $1: 500$ \\
\hline Chk 2(Thr-68) & $62 \mathrm{kDa}$ & Gentex, GTX61178 & $1: 1000$ \\
\hline
\end{tabular}

\subsection{Total RNA extraction}

Prior to use, all solutions and material used were treated overnight with $0.1 \%$ diethylpyrocarbonate (DEPC) and autoclaved twice. Total RNA from liver of $I$.

tridecemlineatus from six stages of the torpor-arousal cycle were isolated using Trizol $^{\mathrm{TM}}$ (BioShop). Briefly, $50 \mathrm{mg}$ of tissue was homogenized in $1 \mathrm{~mL}$ Trizol solution using a Polytron PT1000 homogenizer. Following homogenization, $200 \mu \mathrm{L}$ of chloroform was added, the homogenate was then centrifuged for $10,000 \mathrm{xg}$ for $15 \mathrm{~min}$ at $4^{\circ} \mathrm{C}$. The aqueous layer containing the RNA was removed to a RNase free tube and precipitated by the addition of $500 \mu \mathrm{L}$ isopropanol followed by incubation for $10 \mathrm{~min}$ at room temperature. Upon precipitation, RNA samples were then centrifuged at $10,000 \mathrm{xg}$ for 15 min at $4{ }^{\circ} \mathrm{C}$. RNA pellet was subsequently washed with $1 \mathrm{~mL}$ of $70 \%$ ethanol and centrifuged again at 7,000 $\mathrm{xg}$ for $5 \mathrm{~min}$ at $4{ }^{\circ} \mathrm{C}$. Isolated RNA pellet was air-dried for $15 \mathrm{~min}$ and resuspended in $50 \mu \mathrm{L}$ of DEPC-treated water. RNA quality was assessed based on ratio of absorbances measured at 260 and $280 \mathrm{~nm}$. As well, integrity of $18 \mathrm{~S}$ and $28 \mathrm{~S}$ ribosomal bands was assessed by visualization on a $1 \%$ agarose gel.

\subsection{First strand cDNA synthesis and PCR amplification.}

A $3 \mu \mathrm{g}$ aliquot of RNA was diluted with $7 \mu \mathrm{L}$ of DEPC water and used to synthesize cDNA. The RNA was incubated with $1 \mu \mathrm{L}$ of $200 \mathrm{ng} / \mu \mathrm{L}$ Oligo dT (5'- 
TTTTTTTTTTTTTTTTTTTTTTV-3') primer at $65^{\circ} \mathrm{C}$ for $5 \mathrm{~min}$ and then chilled on ice for $5 \mathrm{~min}$. Subsequent additions of $4 \mu \mathrm{L} 5 \mathrm{X}$ first strand buffer (Invitrogen), $2 \mu \mathrm{L} 100 \mathrm{mM}$ DTT (Invitrogen), $1 \mu \mathrm{L} \mathrm{dNTP} \mathrm{(BioBasic,} \mathrm{mixture} \mathrm{consists} \mathrm{of} 10 \mathrm{mM}$ of each dNTP), and $1 \mu \mathrm{L}$ reverse transcriptase M-MLV (Invitrogen) were made for a total volume of $19 \mu \mathrm{L}$. The mixture was then incubated at $42^{\circ} \mathrm{C}$ for $45 \mathrm{~min}$ and chilled to $4^{\circ} \mathrm{C}$. The cDNA products were diluted to a factor of $10^{-1}$ and $10^{-2}$ and used for the polymerase chain reaction (PCR). The PCR reaction of $25 \mu \mathrm{L}$ was composed of $15 \mu \mathrm{L}$ DEPC water, 1.25 $\mu \mathrm{L}$ of primer mixture (1.5 $\mu \mathrm{M}$ each of forward and reverse primer), $0.75 \mu \mathrm{L}$ of $10 \mathrm{X}$ PCR buffer (Invitrogen), $1.5 \mu \mathrm{L}$ of $50 \mathrm{mM} \mathrm{MgCl}_{2}$ (BioShop), $0.5 \mu \mathrm{L}$ of $25 \mu \mathrm{M}$ dNTP mixture (BioBasic), and $1 \mu \mathrm{L}$ of Taq polymerase. Custom primers were designed from conserved regions in target genes based on DNA alignment with other mammalian species. The sequences of primers used along with annealing temperatures for all target genes are listed below in Table 2.2. A $3 \mu \mathrm{L}$ aliquot of mixture dye (composed of $2 \mu \mathrm{L}$ DNA tracking dye and $1 \mu \mathrm{L}$ of SYBR Green I nucleic acid stain, from Bioshop and Invitrogen, respectively) were added to the PCR products, and products were then separated on a $1.5 \%$ agarose gel, visualized using the ChemiGenius imaging system (Syngene, Frederick, MD, USA) and quantified using the GeneTools program. Gene expressions were then normalized against the housekeeping gene $\alpha$-tubulin measured in the corresponding cDNA sample.

\subsection{Stemloop microRNA PCR}

A $5 \mu \mathrm{g}$ aliquot of RNA was diluted with $5 \mu \mathrm{L}$ of DEPC water and used to synthesize stemploop microRNA. The RNA was initially incubated with $5 \mu \mathrm{L}$ of $200 \mathrm{nM}$ Stemloop 
primer (see Table 2.2 below) at $95^{\circ} \mathrm{C}$ for $5 \mathrm{~min}$ followed by $60^{\circ} \mathrm{C}$ for $5 \mathrm{~min}$. The mixture of then chilled on ice for $5 \mathrm{~min}$, followed by the addition of $8 \mu \mathrm{L}$ of synthesis buffer [containing 4x First Strand buffer (Invitrogen), $100 \mathrm{mM}$ DTT, and $25 \mu \mathrm{M}$ dNTP], $1 \mu \mathrm{L}$ reverse transcriptase M-MLV (Invitrogen), and $6 \mu \mathrm{L}$ of DEPC water. The mixture was then incubated at $16^{\circ} \mathrm{C}$ for $30 \mathrm{~min}$ followed by 60 cycles of: $20^{\circ} \mathrm{C}$ for 30 seconds, $42^{\circ} \mathrm{C}$ for 30 seconds, and $50^{\circ} \mathrm{C}$ for 1 second; and a final step at $85^{\circ} \mathrm{C}$ for 5 minutes. The cDNA products were diluted to a factor of $10^{-1}$ and $10^{-2}$ and used for the polymerase chain reaction (PCR). The PCR reaction of $25 \mu \mathrm{L}$ was composed of $5 \mu \mathrm{L}$ RNA template, $15 \mu \mathrm{L}$ DEPC water, $2 \mu \mathrm{L}$ of primer mixture (a concentration of $1.5 \mu \mathrm{M}$ of forward and universal primer; listed in Table 2.2 below), $0.75 \mu \mathrm{L}$ of 10X PCR buffer (Invitrogen), $1.5 \mu \mathrm{L}$ of 50 $\mathrm{mM}$ of $\mathrm{MgCl}_{2}$ (BioShop), $0.5 \mu \mathrm{L}$ of $25 \mu \mathrm{M}$ of dNTP mixture (BioBasic), and $1 \mu \mathrm{L}$ of Taq polymerase. A $3 \mu \mathrm{L}$ mixture dye (composed of $2 \mu \mathrm{L}$ DNA tracking dye and $1 \mu \mathrm{L}$ of SYBR Green I nucleic acid stain, from Bioshop and Invitrogen, respectively) were added to the PCR products, and separated on a $2.5 \%$ agarose gel, visualized using the ChemiGenius imaging system (Syngene, Frederick, MD, USA) and quantified using the GeneTools program.

Table 2.2. Custom primers sequences designed for target genes, indicated with optimal annealing temperature (Tm) and PCR product size.

\begin{tabular}{|c|c|c|c|}
\hline Gene & Primer sequences & $\begin{array}{c}\text { Anneal } \\
\text { Temp } \\
{ }^{\circ} \mathrm{C}\end{array}$ & $\begin{array}{l}\text { Size } \\
\text { (bp) }\end{array}$ \\
\hline \multicolumn{4}{|l|}{ Chapter 4} \\
\hline PTEN & $\begin{array}{l}\text { Forward 5'-CCAGTCAGAGGCGCTATGTA-3' } \\
\text { Reverse 5'-CTCTGGTCCTGGTATGAAG-3' }\end{array}$ & 55.5 & 580 \\
\hline$P D K-1$ & $\begin{array}{l}\text { Forward 5'-ATGTGATGTCACGCCTGGAT-3' } \\
\text { Reverse 5'-AGTCCTGCCACAAGCTGATA-3' }\end{array}$ & 63.5 & 620 \\
\hline
\end{tabular}




\begin{tabular}{|c|c|c|c|}
\hline \multicolumn{4}{|l|}{ Chapter 5} \\
\hline SMAD2 & $\begin{array}{l}\text { Forward 5'-CTAACAGAATTACCGCCTCTGG-3' } \\
\text { Reverse 5'-GAGACAGTTCTGCTGGAGAGCC-3' }\end{array}$ & 51.4 & 400 \\
\hline SMAD3 & $\begin{array}{l}\text { Forward 5'-CATGTCATCTACTGCCGCCTGT-3' } \\
\text { Reverse 5'-CATCTGGTGGTCGCTGGTCTCT-3' }\end{array}$ & 63.5 & 300 \\
\hline SMAD4 & $\begin{array}{l}\text { Forward 5'-CACCTGGACTGGAAGTAGGA-3' } \\
\text { Reverse 5'-CTCTCCTGTGGACATTGGAG-3' }\end{array}$ & 55.5 & 350 \\
\hline$\alpha$-tubulin & $\begin{array}{l}\text { Forward 5'CCTGCTGATGGAACGTCTCT-3' } \\
\text { Reverse 5'-GTAAGCTGTTCATGGTAGGC-3' }\end{array}$ & 53.2 & 350 \\
\hline Actin- $\beta$ & $\begin{array}{l}\text { Forward 5'-GCATCCTCCTCCTCCCTGGAGAA-3' } \\
\text { Reverse 5'-GTCGACGTCACACTTCATGATGGAA-3' }\end{array}$ & 55.5 & 250 \\
\hline $\begin{array}{c}\text { microRNA } \\
\text { universal }\end{array}$ & $\begin{array}{l}\text { Forward 5'-CTCACAGTACGTTGGTATCCTTGTG- } \\
3 \text { ' }\end{array}$ & 60 & - \\
\hline$m i R-29 b$ & $\begin{array}{l}\text { Forward 5'- } \\
\text { ACACTCCAGCTGGGTAGCACCATCTGAAATC-3' } \\
\text { Stemloop (Reverse) 5'- } \\
\text { CTCACAGTACGTTGGTATCCTTGTGATGTTCGA } \\
\text { TGCCATATTGTACTGTGAGTAACCGAT-3' }\end{array}$ & 42 & 200 \\
\hline$m i R-21$ & $\begin{array}{l}\text { Forward 5'- } \\
\text { ACACTCCAGCTGGGTAGCTTATCAGACTG-3' } \\
\text { Stemloop (Reverse) 5'- } \\
\text { CTCACAGTACGTTGGTATCCTTGTGATGTTCGA } \\
\text { TGCCATATTGTACTGTGAGACAGCCCA-3' }\end{array}$ & 42 & 200 \\
\hline$m i R 155$ & $\begin{array}{l}\text { Forward 5'- } \\
\text { ACACTCCAGCTGGGTTAATGCTAATCGTGAT-3' } \\
\text { Stemloop (Reverse) 5'- } \\
\text { CTCACAGTACGTTGGTATCCTTGTGATGTTCGA } \\
\text { TGCCATATTGTACTGTGAGACCCCTAT-3' }\end{array}$ & 42 & 200 \\
\hline \multicolumn{4}{|l|}{ Chapter 6} \\
\hline Cyclin D1 & $\begin{array}{l}\text { Forward 5'-AGCTCCTGTGCTGCGAAGTG-3' } \\
\text { Reverse 5'-CTTMGAGGCCACGAACATGC-3' }\end{array}$ & 54 & 300 \\
\hline Cyclin A2 & $\begin{array}{l}\text { Forward 5'-CTGAGAATGGAGCACCTYGT-3' } \\
\text { Reverse 5'-AGACTTTCCAGGGTATATCC-3' }\end{array}$ & 53.2 & 250 \\
\hline Cyclin E & $\begin{array}{l}\text { Forward 5'-GATGGTGCTTGTTCAGGAGATG-3' } \\
\text { Reverse 5'-AATCCAAGCTGTTGACGTGG-3' }\end{array}$ & 53.2 & 250 \\
\hline Cyclin B1 & $\begin{array}{l}\text { Forward 5'-GCCATGTTTATTGCAAGCAA-3' } \\
\text { Reverse 5'-TTCATYTCCATCTGTCTGAT-3' }\end{array}$ & 57.5 & 100 \\
\hline
\end{tabular}




\begin{tabular}{|c|l|c|c|}
\hline$p 21$ & $\begin{array}{l}\text { Forward 5'-ATTGCGATGCSCTCATGGCKG-3' } \\
\text { Reverse 5'-TGCAGCAGGGCRGATGARGT-3' }\end{array}$ & 69.1 & 200 \\
\hline$p 15$ & $\begin{array}{l}\text { Forward 5'-CAGGTCATGATGATGGGCAGC-3' } \\
\text { Reverse 5'-CCAGGCATCGCGCACGTCCAG-3' }\end{array}$ & 68.5 & 200 \\
\hline
\end{tabular}

\subsection{Electrophoretic mobility shift assay}

Aliquots of $5 \mu \mathrm{g}$ of nuclear extract was incubated with $1 \mu \mathrm{g}$ of poly $\mathrm{d}(\mathrm{I}-\mathrm{C}), 2 \mu \mathrm{L}$ of EMSA 5x binding buffer (50 mM Tris, $\mathrm{pH} 7.8,250 \mathrm{mM} \mathrm{NaCl}, 5 \mathrm{mM}$ EDTA, 25\% glycerol) and $5 \mu \mathrm{L}$ of nuclease-free water for $5 \mathrm{~min}$ at room temperature in $0.2 \mathrm{~mL}$ microcentrifuge tubes. Using biotinylated DNA probes corresponding to the Smad binding element (Forward: biotin-5'-AGTATGTCTAGACTGA-3', Reverse: 5'TCAGTCTAGACATACT-3'), 250 pmoles of probe was added to this mixture, and incubated at $15^{\circ} \mathrm{C}$ for 30 min using a thermal cycler. Subsequently, for an antibody shift sample, $1 \mu \mathrm{L}$ of stock antibody $(1 \mu \mathrm{g} / \mu \mathrm{L})$ was added to the mixture and incubated for an additional $1 \mathrm{~h}$ at room temperature. Samples were then separated on a $6 \%$ non-denaturing polyacrylamide gel in $0.5 \mathrm{X}$ TBE buffer (89mM Tri-borate, $\mathrm{pH} 8.3,2 \mathrm{mM}$ EDTA) for 30 min at $120 \mathrm{~V}$. Proteins/oligonucleotides on the gel were then wet-transferred onto a nylon membrane in $0.5 \mathrm{X}$ TBE for $30 \mathrm{~min}$ at $300 \mathrm{~mA}$ and then the nylon membrane was incubated for $1 \mathrm{~h}$ in a dry oven at $80^{\circ} \mathrm{C}$ to fix the oligonucleotides onto the nylon membrane. The membrane was then blocked in $5 \mathrm{~mL}$ of Blocking buffer (Panomics, \# CS7537) for $15 \mathrm{~min}$, followed by addition of $5 \mu \mathrm{l}$ of Streptavidin-HRP directly into the blocking buffer and incubation for an additional $15 \mathrm{~min}$. Membranes were then washed in $1 \mathrm{X}$ wash buffer for $3 \times 10$ min intervals. Membranes were visualized using same procedures as described for Western blots. 


\subsection{DNA-binding assessed via enzyme-linked immunosorbent assay}

A 40 pmol aliquot of double stranded biotinylated DNA corresponding to the Smad binding element, previously described above, was prepared in phosphate buffered saline (PBS; $137 \mathrm{mM} \mathrm{NaCl}, 2.7 \mathrm{mM} \mathrm{KCl}, 10 \mathrm{mM} \mathrm{Na}_{2} \mathrm{HPO}_{4}, 2 \mathrm{mM} \mathrm{KH}_{2} \mathrm{PO}_{4}, \mathrm{pH}$ 7.4), and then $50 \mu \mathrm{L}$ was added to each well of a streptavidin coated 96 well microplate and incubated overnight. After incubation, the plate was washed twice with wash buffer (0.1\% Tween-20 in PBS, PBST) and once in PBS. Using nuclear extracts prepared as described above, $10 \mu \mathrm{g}$ aliquots of nuclear proteins were added to the wells on coated and non-coated (negative control) microplates along with $1 \mathrm{X}$ protein binding buffer $(10 \mathrm{mM}$ HEPES, pH 7.9, 50 mM KCl, 0.5 mM EDTA, 3 mM MgCl2, 10\% glycerol, 0.5 mg/mL BSA, $0.05 \%$ NP-40, $1.5 \mu \mathrm{g}$ salmon sperm DNA, $0.5 \mathrm{mM}$ DTT, and $40 \mathrm{mM} \mathrm{NaCl}$ ) and incubated for $1 \mathrm{~h}$ with shaking at $100 \mathrm{rpm}$. Negative controls were also carried out using $10 \mu \mathrm{L}$ of PBS buffer instead of protein extracts. Post incubation, plates were washed four times with PBST followed by $60 \mu \mathrm{L}$ of antibody for the transcription factor of interest diluted in 1:1000 PBST for $1 \mathrm{~h}$. The plate was then washed four times in PBST, and coated with secondary antibody anti-rabbit IgG-HRP (BioShop) diluted 1:4000 in PBST for $1 \mathrm{~h}$. The plate was then washed four more times, and incubated with $60 \mu \mathrm{L}$ of tetramethylbenzidine (TMB) for 15-30 min in the dark (depending on the colour development). Once colour development has taken place, the reaction was stopped using $1 \mathrm{M} \mathrm{HCl}$ and absorbance readings were measured at $\mathrm{A}_{450} \mathrm{~nm}$ with a reference absorbance at $\mathrm{A}_{655} \mathrm{~nm}$ using a Multiskan Spectrum (Thermo Labsystems). 


\subsection{Co-Immunoprecipitation}

Chemical cross-link of Smad 3 polyclonal antibody was first performed in order to avoid detection of antibody heavy chain at $50 \mathrm{kDa}$ in subsequent Western blot procedures (interfering with detection of Smad 3 at 52kDa). Protein A agarose beads were first centrifuged at $500 x \mathrm{~g}$ for $5 \mathrm{~min}$, followed by equilibration with PBS at a 1:1 v: $\mathrm{v}$ ratio on a rotisseries overnight at $4^{\circ} \mathrm{C}(200 \mu \mathrm{L}$ of bead and PBS $)$. The equilibrated bead slurry was then centrifuged at $500 x \mathrm{~g}$ for 5 min with supernatant discarded afterwards. Aliquot of $20 \mu \mathrm{L}$ of Smad 4 primary antibody $(1 \mu \mathrm{g} / \mu \mathrm{L})$ was diluted in PBS to a final volume of $200 \mu \mathrm{L}$, followed by incubation with protein A beads at a 1:1 ratio for $2 \mathrm{~h}$ at $4^{\circ} \mathrm{C}$ on a rotisserie. Bead slurry are then centrifuged with supernatants discarded, followed by cross linking with diemthyl pimelimidate (DMP, $13 \mathrm{mg} / \mathrm{mL}$ ) at a 1:1 ratio and incubated on a rotisserie for $30 \mathrm{~min}$ at room temperature. Crosslink with DMP was then repeated for three times with PBS washing between each step. Bead slurry was then incubated with quench buffer (50 $\mathrm{mM}$ ethanolamine in PBS) at a 1:1 ratio for $5 \mathrm{~min}$ at RT, and repeated twice more. Excess unlinked antibody are removed by washing the beads with $1 \mathrm{M}$ glycine $(\mathrm{pH} 3)$ at a 1:1 ratio, and repeated twice more to ensure complete removal of unlinked Smad 4 antibody. Crosslinked antibody was then equilibrated with PBS before incubation with tissue lysates. Aliquot of $500 \mu \mathrm{g}$ of proteins (tissue extracts were prepared as described in $\mathbf{2 . 2}$ ) was then incubated with $50 \mu \mathrm{L}$ of bead: antibody slurry and incubated overnight on the rotisserie at $4{ }^{\circ} \mathrm{C}$. The slurry was then centrifuged at $500 x \mathrm{~g}$ for $5 \mathrm{~min}$ to remove the supernatant, followed by additional of 2X SDS buffer at 1:1 ratio and boiled for $5 \mathrm{~min}$. Western immunoblot was then performed as described in 2.2, and detected for presence of Smad 4, p-Smad 3, and Smad 3. 


\subsection{Protein isolation for phosphatase assay}

Samples of frozen I. tridecemlineatus skeletal muscle were homogenized 1:5 w:v in cold $\left(4^{\circ} \mathrm{C}\right)$ homogenization buffer containing $25 \mathrm{mM}$ HEPES, $\mathrm{pH} 7.5,10 \%$ v:v glycerol, 2 mM EDTA, 2 mM EGTA, and 10 mM 2-mercaptoethanol, using a Polytron PT1000 homogenizer (Brinkmann Instruments, Rexdale, ON, Canada). A few crystals of PMSF were added at the time of homogenization. Homogenates were centrifuged at 10,000 x $g$ for $30 \mathrm{~min}$ at $4^{\circ} \mathrm{C}$ and then the supernatant was removed and centrifuged through small columns of Sephadex G-50 to endogenous remove ions and metabolites. Before use, the G-50 columns were equilibrated in homogenization buffer and centrifuged in a bench-top IEC clinical centrifuge at 2,000 rpm for $2 \mathrm{~min}$; crude supernatant was then layered onto the column followed by a 1 min centrifugation. The eluant was collected and stored on ice until use. Protein concentration was determined by the Bradford assay (Bio-Rad, Hercules, CA, USA).

\subsection{PTEN phosphatase assay}

PTEN activity was assayed by measuring the rate at which diC8phosphatidylinositol 3,4,5-trisphosphate $\left(\mathrm{PIP}_{3}\right)$ is dephosphorylated. One unit of activity is defined as the amount of enzyme that releases one nmol of phosphate per min.

Standard assay conditions in $40 \mu \mathrm{L}$ reaction volumes contained $25 \mathrm{mM}$ Tris, $\mathrm{pH} 7.5,5 \mu \mathrm{L}$ of crude homogenate, and $25 \mu \mathrm{M}$ of soluble $\mathrm{PIP}_{3}$ (Echelon, P-3908). For $\mathrm{K}_{\mathrm{m}}$ determinations, activity was assayed between $0-45 \mu \mathrm{M}$ of $\mathrm{PIP}_{3}$. Standard reaction time was $60 \mathrm{~min}$ at $37^{\circ} \mathrm{C}$. Parallel assays were run in the presence versus absence of $300 \mathrm{~nm}$ of VO-OHpic trihydate, a potent PTEN inhibitor (Santa Cruz Biotechnology, sc-216061) 
and PTEN activity was defined as activity towards its substrate that was abolished in the presence of $300 \mathrm{~nm}$ of VO-OHpic. After incubation, reactions were quickly diluted with $\mathrm{ddH}_{2} \mathrm{O}$ to a volume of $800 \mu \mathrm{L}$, and then $200 \mu \mathrm{L}$ of ammonium molybdate/malachite green dye reagent $(2.5 \% \mathrm{w}: \mathrm{v}$ ammonium molybdate, $0.05 \% \mathrm{w}: \mathrm{v}$ malachite green in $4 \mathrm{M}$ $\mathrm{HCl}$ ) was immediately added. Reaction tubes were then stored in the dark to allow colour development for $15 \mathrm{~min}$. Subsequently, aliquots of $200 \mu \mathrm{L}$ were transferred into microplate wells and absorbance at $600 \mathrm{~nm}$ was read using a MR5000 microplate reader with Biolinx 2.0 software. The mean absorbance of blank samples (no peptide) was subtracted and then the amount of free phosphate was determined by comparison with a standard curve. Activity of PTEN was defined as units (nmol phosphate / min) normalized with relative amount of PTEN present in the corresponding sample as determined by Western blotting.

\subsection{Purification of PTEN using Heparin agarose}

A $500 \mu \mathrm{L}$ aliquot of muscle extracts prepared as described above were applied to a $3 \times 1.8 \mathrm{~cm}(\mathrm{~h} \times \mathrm{d})$ column of Heparin agarose (Sigma Aldrich, HEPI5-1KT ) equilibrated in homogenization buffer. Columns were washed with $\sim 10 \mathrm{~mL}$ of homogenization buffer to remove unbound proteins, PTEN proteins were then eluted using a 0-2 $\mathrm{M} \mathrm{KCl}$ gradient

of homogenization buffer. Fractions of $500 \mu \mathrm{L}$ were collected and the elution of profile of PTEN was assessed through Western blotting. Eluted fractions containing PTEN proteins were then concentrated using an Amicon Ultra-4 centrifugal filter unit with ultracel-3 membrane (Millipore, UFC800324). Four to six fractions containing PTEN proteins were centrifuged at $4000 \times \mathrm{g}$ for $15 \mathrm{~min}$ in the Amicon concentrator, where a concentrated sample of $\sim 1 \mathrm{~mL}$ was then collected. 


\subsection{Pulse Proteolysis}

Purified PTEN protein samples were tested for structural stability via pulse proteolysis assay. A $20 \mu \mathrm{L}$ aliquot of purified PTEN samples were incubated with various concentrations of urea prepared in homogenization buffer $(0,0.5,1,1.25,1.5$, $1.75,2,2.25,2.5,2.75,3,4$, and $5 \mathrm{M}$ of urea) with a final volume of $60 \mu \mathrm{L}$ for $2 \mathrm{~h}$ at room temperature. Proteolysis was initiated with the addition $2 \mu \mathrm{L}$ of thermolysin (prepared at a concentration of $10 \mathrm{mg} / \mathrm{mL}$ in $2.5 \mathrm{M} \mathrm{NaCl}$ and $10 \mathrm{mM} \mathrm{CaCl}_{2}$ ) from Bacillus thermoproteolyticus rokko (Sigma Aldrich, 88303) into $30 \mu \mathrm{L}$ of urea incubated samples. Proteolysis was allowed to proceed for $10 \mathrm{~min}$, after which the reaction was quenched with the addition of $18 \mu \mathrm{L}$ of $50 \mathrm{mM}$ EDTA ( $\mathrm{pH} 8.0)$. Samples were then mixed with $50 \mu \mathrm{L}$ of $2 \mathrm{X}$ SDS-PAGE sample buffer and boiled. Samples were then separated on a $10 \%$ SDS-PAGE gel at $180 \mathrm{~V}$ for $45 \mathrm{~min}$. SDS-PAGE gels were then visualized using silver staining to assess the rate of PTEN degradation.

\subsection{Silver staining}

After electrophoresis, the SDS-PAGE gels were fixed in fixing solution $(50 \% \mathrm{v} / \mathrm{v}$ ethanol, $12 \% \mathrm{v} / \mathrm{v}$ acetic acid, $0.05 \% \mathrm{v} / \mathrm{v}$, and 35\% formaldehyde) for $2 \mathrm{~h}$ followed by three rounds of washing in $20 \%$ ethanol for $20 \mathrm{~min}$. The gels were then sensitized in sensitizing solution $\left(0.02 \% \mathrm{w} / \mathrm{v}\right.$ sodium thiosulphate $\left.\left(\mathrm{Na}_{2} \mathrm{~S}_{2} \mathrm{O}_{3}\right)\right)$ for 2 min with gentle rotation followed by two rounds of washing with $\mathrm{ddH}_{2} \mathrm{O}$ for 1 min each. Freshly prepared and chilled silver stain solution $(0.6 \% \mathrm{w} / \mathrm{v}$ silver nitrate, $0.076 \% \mathrm{v} / \mathrm{v}$ formalin) was then added to the gel for a 20 min incubation with gentle shaking. Silver stain was then discarded and washed twice with $\mathrm{ddH}_{2} \mathrm{O}$ for $1 \mathrm{~min}$, followed by incubation with 
developing solution $\left(6 \% \mathrm{w} / \mathrm{v}\right.$ sodium carbonate $\left(\mathrm{Na}_{2} \mathrm{CO}_{3}\right), 0.04 \% \mathrm{w} / \mathrm{v}$ sodium thiosulphate $\left(\mathrm{Na}_{2} \mathrm{~S}_{2} \mathrm{O}_{3}\right)$, and $0.05 \% \mathrm{v} / \mathrm{v}$ formalin) for $2-5 \mathrm{~min}$, depending on the rate of reduction. The reaction was terminated with the addition of $12 \%$ acetic acid, and the gel was visualized using the ChemiGenius Bio-Imaging System, followed by densitometry analysis of protein bands using the GeneTools software.

\subsection{PEST motif prediction}

The amino acid sequence of PTEN from the thirteen-lined ground squirrel (Ictidomys tridecemlineatus) was retrieved from the ensemble bioinformatics database (ENSSTOG00000022936). Sequence was analyzed for potential PEST motif using the web based bioinformatics program epestfind (http://emboss.bioinformatics.nl/cgibin/emboss/epestfind).

\subsection{Sequence comparisons for PCR amplicons}

Amplified PCR products are converted into amino acids matching the open reading frames of the same gene in sequenced animals of human, mouse, and rats. Alignments were done using DNAMAN alignment tool (Pointe-Claire, Quebec, Canada). Sequence of human, mouse, and rate genes were retrieved from NCBI, with accession numbers list in Table 2.3 below.

Table 2.3. Accession numbers of sequences retrieved from NCBI nucleotide collection used for homology alignment comparisons.

\begin{tabular}{|c|l|l|l|}
\hline Gene & Homo sapiens & Mus musculus & Rattus norvegicus \\
\hline Chapter 4 & BC021445.1 & AF455569.1 \\
\hline PTEN & AH007803.1 & & \\
\end{tabular}




\begin{tabular}{|c|l|l|l|}
\hline PDK-1 & AF017995.1 & AY062008.1 & NM_031081.1 \\
\hline Chapter 5 & BC021342.1 & AB010147.1 \\
\hline SMAD2 & U78733.1 & AF016189.1 & U66479.1 \\
\hline SMAD3 & AH011390.1 & AY119788.1 & AB010954.1 \\
\hline SMAD4 & BC002379.2 & BC044841.1 & BC044841.1 \\
\hline Chapter 6 & BC052730.1 & BC127462.1 \\
\hline Cyclin D1 & BC023620.2 & X75888.1 & D14015.1 \\
\hline Cyclin A2 & BC104783.1 & BC011478.1 & BC059113.1 \\
\hline Cyclin E & NM_001238.2 & U22399.1 & AY576608.1 \\
\hline Cyclin B1 & BC006510.2 & BC002010.1 & AF474978.1 \\
\hline$p 21$ & BC000312.2 & & \\
\hline$p 15$ & L36844.1 &
\end{tabular}




\section{CHAPTER 3}

\section{REGULATION OF mTOR SIGNALING NETWORK IN HIBERNATING GROUND SQUIRRELS}

Published in J. Exp. Biol. Reprint with permission of Company of Biologists LTD. 


\subsection{INTRODUCTION}

Metabolic processes that promote cellular growth require the increase of cell size

and number, which involves the synthesis of new biological compartments comprised of the four major macromolecules: nucleic acid, protein, carbohydrates and lipids. The cellular cues such as extracellular stimuli and nutrient availability enable the cells to determine whether a favourable environment is in place for promotion of cell growth. One of the mechanisms for such detection functions through a signaling pathway that is centered on the target of rapamyacin (TOR) kinase (Zoncu et al., 2010). In multicellular organisms, the TOR kinase primarily functions as a regulator of cellular growth and homeostasis, acting downstream of the insulin signaling pathway, and is best characterized for its role in regulation of protein translation.

One of the most energy-expensive processes in cells is protein synthesis and this is a key target for regulation in all forms of hypometabolism (Storey and Storey, 2007, 2010). Indeed, Frerichs et al. (1998) showed that the rate of protein synthesis in vivo in brain of torpid ground squirrels brain was just $0.04 \%$ of that in active squirrels. In the same study, ribosomal disaggregation along with increased eIF2- $\alpha$ phosphorylation during hibernation was also observed, reinforcing a decreased capacity for the initiation of protein synthesis (Frerichs et al. 1998). The molecular mechanisms that control protein synthesis are well-studied. The translational machinery responds to multiple extracellular signals (e.g. insulin, mitogenic growth factors) that stimulate the phosphoinositide 3-kinase (PI3-K) / Akt / mTOR pathway (Ruggero and Sonenberg, 2005; Yang and Quan, 2007). As well, the mTOR signaling pathway responds to other 
stimuli such as energy status (via the AMP-activated protein kinase) and amino acid availability (through interaction with Rag GTPase) (Yang and Quan, 2007; Kim et al., 2008).

The mTOR signaling pathway integrates inputs from several kinase cascades that are themselves responsive to different inputs (e.g. amino acid or energy availability, growth factors, etc.) (Tokunaga et al., 2004). mTOR is able to form two independent complexes, mTORC1 and $\mathrm{mTORC} 2$, both with distinct properties and functions that are characterized based on their interacting proteins (Foster and Fingar, 2010). The mTORC1 complex plays a major role in regulating cellular growth by promoting protein synthesis via the activation of p70S6 kinase (p70S6K) and inhibition of the eukaryotic translation initiation factor 4E-binding protein 1 (4E-BP1) (Ma and Blenis, 2009). Through downstream targets of p70S6K and 4E-BP1, mTORC1 regulates the rate of ribosomal binding and assembly, which is considered to be the rate limiting process of translational initiation (Matthews et al., 2000). The regulation of mTORC1 is of particular interest due to its ability to regulate energy consumption based on nutrient availability, which is especially critical during the non-feeding hibernation season when availability of amino acids for new protein synthesis is limited. mTOR is part of a signal transduction pathway that is initiated at the insulin receptor, with Akt being a direct upstream regulator (FIG. 3.1). The role of Akt in mTOR regulation was established based on its ability to phosphorylate and inactive the TSC1-TSC2 complex, a well-known inhibitor of mTOR kinase activity (Inoki et al., 2002). The TSC1-TSC2 complex forms a GTPase activating protein (GAP) that stimulates the intrinsic activity of Rheb:GTP, catalyzing the cleavage of Rheb:GTP to form Rheb:GDP (FIG. 3.1) (Huang and Manning, 2008). The conversion 
to a GDP-bound Rheb results in the loss of its GTPase activity, and the loss of its ability to stimulate mTORC1, rendering mTORC1 inactive. However, under a state of growth promotion, activated Akt inhibits the TSC1-TSC2 complex through phosphorylation of TSC2 at Thr-1462 (McManus et al., 2002). The Akt mediated phosphorylation of TSC2 (Thr-1462) inhibits the function of the TSC1-TSC2 complex by destabilizing the TSC2 proteins, and disrupting complex stability (Inoki et al., 2002). More recently, the discovery of Akt regulation of a proline-rich Akt substrate of $40 \mathrm{kDa}$ (PRAS40), an inhibitor of the mTORC1 complex, suggests that Akt is able to regulate mTOR kinase activity through multiple effectors (McMullen and Hallenbeck, 2010). Akt stimulates the activation of mTORC1 via phosphorylation of PRAS40, an interacting partner of regulatory associated protein of TOR (RAPTOR) (McMullen and Hallenbeck, 2010). PRAS40 inhibits the mTORC1 complex by sequestering RAPTOR, inhibiting the interaction of mTORC1 and its downstream targets, p70S6K and 4E-BP1. However, upon Akt-mediated phosphorylation of PRAS40 (Thr-246), PRAS40 loses affinity for RAPTOR, allowing the restoration of the mTOC1 complex (McMullen and Hallenbeck, 2010).

Regulation of translational capacity has been linked to the mTOR signaling network in a wide range of species (e.g. yeast, nematodes, flies, mammals) (reviewed in Hay and Sonenberg, 2004). Previous studies have also shown that environmental stresses such as hypoxia (which limits ATP availability) cause inhibition of mTOR signaling which leads to suppression of protein synthesis via disruption of cap-dependent initiation of translation (Connolly et al., 2006). The present chapter provides biochemical data that demonstrates that reversible regulation of the mTOR signaling network also occurs over 
the torpor-arousal cycle of hibernation providing a means of global protein synthesis inhibition during torpor. Furthermore, the data show that the mTOR signaling network is differentially regulated in two muscle types; mTOR is negatively regulated in skeletal muscle during torpor, but maintained at euthermic levels in cardiac muscle. This pattern of mTOR regulation in the two muscle types reflects their physiological states during hibernation - an atrophy-susceptible skeletal muscle versus a cardiac muscle that undergoes hypertrophy (Wickler et al., 1991). Overall, this study provides new insights into the molecular mechanisms that can regulate muscle protein synthesis during ground squirrel hibernation.

\subsection{RESULTS}

\subsection{1 mTOR and phosphorylated mTOR status during hibernation}

The mTOR signaling pathway plays a critical role in regulating cellular growth and proliferation by directly regulating protein synthesis. Previous studies have shown that mTOR is a central player in a kinase cascade that promotes ribosome assembly in response to a variety of stimuli (Wang and Proud, 2006). Total levels of mTOR protein (immunoreactive band at $290 \mathrm{kDa}$ ) were compared under two conditions: euthermic in the cold room (EC) and late torpor (LT; $>5$ days constant torpor). Western blots showed no significant changes in total protein levels of mTOR in either cardiac (mean normalized values were $1.00 \pm 0.07$ vs. $0.82 \pm 0.07$ relative band intensity units in EC vs. LT) or skeletal $(1.00 \pm 0.17$ vs. $0.84 \pm 0.12)$ muscle between the two states (FIG. 3.2). However, mTOR is well-known to be regulated by phosphorylation at Ser-2448 and so relative changes in phosphorylation state were monitored over the six torpor-arousal 
stages. FIG. 3.3 shows changes in p-mTOR Ser-2448 in skeletal muscle. Relative pmTOR Ser-2448 levels were unchanged during entrance $(E N)$ and early torpor $(<24 \mathrm{~h})$ but decreased by 55\% during LT. However, during arousal from torpor (EA), p-mTOR Ser-2448 increased rapidly to 2.0-fold higher than EC values (or 4-fold higher than LT) $(\mathrm{P}<0.05)$ before beginning to decline during interbout (IA). Cardiac muscle showed a different pattern (FIG. 3.4). p-mTOR Ser-2448 content increased strongly during EN (by 2.9-fold; $\mathrm{P}<0.05$ ), decreased again during torpor, and then rose strongly by 3.2 -fold during arousal $(\mathrm{P}<0.05)$ before declining in IA.

\subsubsection{Phosphorylation of upstream regulators of mTOR over torpor-arousal}

To determine if differential phosphorylation of mTOR over the course of torporarousal was controlled by upstream regulators, the phosphorylation state of TSC2, a known inhibitor of mTOR phosphorylation, was assessed. Phosphorylation of Thr-1462 on TSC2 is regulated by Akt to destabilize the TSC1-TSC2 dimer, an inhibitory complex of mTOR phosphorylation. FIG.3.3 shows that in skeletal muscle the relative level of phosphorylation of the $200 \mathrm{kDa}$ TSC2 at Thr-1462 was unchanged over most of the torpor-arousal cycle except for a $51 \%$ decrease during LT as compared to $\mathrm{EC}(\mathrm{P}<0.05)$, a response that paralleled that of p-mTOR Ser-2448. However, in cardiac muscle, p-TSC2 Thr-1462 content did not change over the torpor-arousal cycle (FIG 3.4). To determine if Akt was a potential mediator of TSC2 Thr-1462 phosphorylation in LT, the relative phosphorylation of Akt was assessed at Thr-473. FIG. 3.5 shows that p-Akt Thr473 (60 $\mathrm{kDa}$ ) content decreased strongly by $55 \%$ in skeletal muscle during LT, compared to EC $(\mathrm{P}<0.05)$ but was not affected in cardiac muscle. 


\subsubsection{Regulation of the mTORC1 complex}

The mTORC1 multi-protein complex is responsible for regulating the formation of active ribosomal complexes for protein translation. To assess the status of the mTORC1 complex during hibernation, two key interacting proteins in the mTORC1 complex were evaluated: PRAS40 and G $\beta$ L. PRAS40 interacts with RAPTOR in the mTORC1 complex and inhibits the mTORC1 complex when dephosphorylated. As shown in FIG. 3.3 the relative phosphorylation level of p-PRAS40 on Thr-246 (40 kDa) in skeletal muscle increased significantly by 1.2 -fold during EN, then fell during torpor and rose again by 1.4-fold during EA. However, in cardiac muscle, significant changes in the phosphorylation state of PRAS40 Thr-246 occurred only in EA where levels increased by 1.9- fold during EA before returning to near-control levels in IA (FIG. 3.4). Another critical subunit of the mTORC1 complex is $\mathrm{G} \beta \mathrm{L}$, a protein that stimulates mTOR kinase activity. FIG. 3.3 shows that total levels of $\mathrm{G} \beta \mathrm{L}(37 \mathrm{kDa})$ in skeletal muscle increased significantly by 1.8 -fold during EN, compared with EC, but were then strongly reduced when torpor was established, decreasing to $69 \%$ of the EC value during LT. However, during arousal, $\mathrm{G} \beta \mathrm{L}$ content quickly rose again in EA to 1.5 -fold higher than the control value before returning to near-EC values during interbout. In cardiac muscle, $\mathrm{G} \beta \mathrm{L}$ total protein was relatively stable throughout the hibernation cycle, showing no significant changes from the EC values (FIG. 3.4).

\subsubsection{Phosphorylation of downstream targets of mTOR.}

To assess the effects of differentially regulated mTOR signaling over the torporarousal cycle, the phosphorylation status of two well-known mTORC1 downstream 
targets were evaluated as indicators of mTORC1 kinase activity: p-4E-BP1 Thr-36 and pp70S6K Thr-389. Both 4E-BP1 and p70S6K are known to play major roles in the assembly of the translation complex, and act as the major mechanism of mTOR mediated translational control. FIG. 3.6 shows that in skeletal muscle, the relative level of p-4EBP1 Thr-36 ( 17 kDa) was strongly reduced during torpor, falling by $66 \%$ and $72 \%$ in during ET and LT, respectively, as compared to EC $(\mathrm{P}<0.05)$. However, FIG. 3.7 shows that the phosphorylation state of 4E-BP1 Thr-36 in heart was unchanged during EN and throughout torpor but increased strongly by 1.8 -fold during EA, as compared to EC $(\mathrm{P}<0.05)$. Levels returned to near control values during interbout.

The relative phosphorylation of p70S6K Thr-389 $(70 \mathrm{kDa})$ in skeletal muscle was unchanged over EN and torpor but increased significantly by 2.0 -fold during EA $(\mathrm{P}<0.05)$ before returning to control levels in IA (FIG. 3.6). Cardiac muscle showed the same pattern of stable levels over EN, ET and LT with relative phosphorylation of p70S6K Thr-389 rising by 2.4-fold during EA, as compared with EC $(\mathrm{P}<0.05)$ before dropping again in IA (FIG 3.7). To determine if changes in the phosphorylation state of p-p70S6K Thr-389 influenced the formation of the translational complex, the phosphorylation state of the S6 ribosomal protein on Ser-235 was also evaluated. In skeletal muscle, phosphorylation of S6 Ser-235 (32 kDa) decreased significantly during torpor by $43 \%$ and $41 \%$ in ET and LT, respectively, compared to EC $(\mathrm{P}<0.05)$ (FIG. 3.6). Levels returned to control values during arousal. In cardiac muscle, however, phosphorylation of S6 Ser-235 was unchanged throughout torpor but increased by 1.4fold in EA compared to $\mathrm{EC}(\mathrm{P}<0.05)$ before decreasing again in IA (FIG 3.7). 


\subsection{DISCUSSION}

The ATP demand of protein synthesis consumes $12-25 \%$ of total energy expenditure in mammalian muscle (Kelly and McBride, 1990; Rolfe and Brown, 1997). Reducing this energetically expensive cellular process can make an important contribution to global metabolic rate depression during hibernation and other states of hypometabolism (Frerichs et al., 1998; Storey, 2010). The mTOR signaling pathway regulates 5' cap-dependent protein synthesis in mammals, the route that accounts for the majority of cellular translation (Carrera, 2004). Through regulation of 4E-BP1, a direct downstream substrate of mTOR, cap-dependent protein synthesis is inhibited via attenuation of eukaryotic translation initiation factor 4E (eIF4E) action in translational initiation (van Breukelen et al., 2004). Since the mTOR signaling pathway is a major regulator of cap-dependent protein synthesis, it can be suggested that this pathway may be subject to reversible regulation during torpor in order to limit nonessential ATP consuming processes.

\subsubsection{Post-translational regulation of mTOR upstream targets}

A major upstream regulator of the mTOR signaling pathway is Akt, a central protein kinase that is involved in regulating a wide variety of cellular processes including cell survival and proliferation (Song et al., 2005). Previous studies have shown that Akt activity is suppressed during torpor in multiple tissues of ground squirrels (Cai et al., 2004; Abnous et al., 2008) and the current study concurs. FIG. 3.5 shows that the amount of active phosphorylated p-Akt Ser-473 was reduced by about one-half in skeletal muscle, but Akt was unaffected in heart during long term torpor. Akt is only active in its 
phosphorylated form and so reduced Akt phosphorylation in skeletal muscle suggests a strong reduction of pro-Akt mediated processes during torpor. This was indeed observed in the present study. FIG. 3.3 shows that the phosphorylation states of two Akt downstream effectors p-TSC2 Thr-1462 and p-mTOR Ser-2448 were both strongly reduced during late torpor in skeletal muscle. This would lead to inhibition of the mTORC1 complex by two mechanisms: allowing inhibition of the complex by dephosphorylated TSC2 and reducing mTOR kinase activity due to reduced phosphorylation of the Ser-2448 activating site. Furthermore, the overall pattern of change in p-mTOR Ser-2448 content over the torpor-arousal cycle in skeletal muscle generally mimicked that of p-TSC2 Thr-1462, suggesting that the reduction in mTOR phosphorylation depended on the phosphorylation status of p-TSC2 Thr-1462 that is, in turn, regulated by Akt. Previous studies have found that overexpression of TSC2 results in inhibition of mTOR phosphorylation in a dose-dependent manner and it has been suggested that TSC2 mediates the inhibition of mTOR phosphorylation in a competitive manner, by blocking the accessibility of mTOR to Akt (Inoki et al., 2002). In cardiac muscle, however, the phosphorylation patterns of p-TSC2 Thr-1462 and p-mTOR Ser2448 were different over the course of torpor. Levels of p-mTOR Ser-2448 showed two distinct peaks in EN and EA but the relative amount of p-TSC2 Thr-1462 remained steady during EN (and torpor) although showing an elevated trend during EA (FIG. 3.4). This may suggest that there are other regulatory influences on cardiac mTOR over the torpor-arousal cycle. Cardiac muscle showed two peaks of increased p-mTOR Ser-2448 content ( $\sim 3$-fold) during entrance into and arousal from torpor whereas skeletal muscle showed elevated p-mTOR Ser-2448 only during arousal along with a strong depression 
during long term torpor (FIG. 3.3, FIG. 3.4). Overall, the evidence supports the action of Akt as a direct upstream regulator of $\mathrm{mTOR}$, and the reduction in Akt phosphorylation during torpor as a key influence on the activity of the mTORC1 complex, potentially leading to a depression of cap-dependent protein synthesis during torpor. The proposed mechanism of regulation is a phosphorylation cascade that alters the activity states of mTOR and its regulators since total protein expression levels of mTOR were stable between euthermia and hibernation (FIG. 3.2).

\subsubsection{Regulation of mTORC1 complex}

To expand the analysis of the regulation of the mTORC1 complex in hibernation, I next assessed the phosphorylation level of PRAS40 along with the protein expression of G $\beta \mathrm{L}$; these are two integral components of the mTORC1 complex (Kim et al, 2003; Vander Haar et al., 2007). Relative phosphorylation levels of p-PRAS40 Thr-246 were elevated during entrance and arousal stages of hibernation in skeletal muscle, and during the arousal stage in heart (FIG. 3.3, FIG. 3.4). It has previously been shown that Akt mediated phosphorylation of PRAS40 at Thr-246 leads to its binding with the chaperone protein 14-3-3, inhibiting the ability of PRAS40 to block interactions between mTORC1 and its substrates (Kovacina et al., 2003; Wang et al., 2008). The observed increase in phosphorylation of p-PRAS40 Thr-246 in both skeletal and cardiac muscle suggests that the inhibitory effects of PRAS40 on mTORC1 are minimized during the heterothermic phases of hibernation when the animal is transitioning into or out of torpor. Similar patterns were observed in the expression of GßL with enhanced protein levels observed during entrance and arousal stages of hibernation in skeletal muscle (but not in heart). 
$\mathrm{G} \beta \mathrm{L}$ is a subunit of the mTORC1 complex that positively regulates the mTOR signaling pathway via stimulation of mTOR kinase activity (Kim et al., 2003). An upregulation of G $\beta L$ expression along with the decrease in PRAS40 inhibitory effects would potentiate mTORC1 activity in skeletal muscle during the entrance and arousal stages of hibernation. These findings coincide with those of McMullen and Hallenbeck (2010) for I. tridecemlineatus liver where an activation of mTOR was seen during both entrance and arousal stages of hibernation. These findings are plausible considering that the entry and arousal stages are arguably the most critical phases of the hibernation cycle. Enhanced mTORC1 phosphorylation during the entry stage could support an increase in the synthesis of selected proteins that are critical to long term survival in torpor. Indeed, previous studies on ground squirrels have identified a number of proteins that are selectively upregulated during torpor including glucose-regulated protein 78 , peroxisome proliferator-activated receptors- $\gamma$ (PPAR- $\gamma$ ), PPAR- $\gamma$ coactivator $1-\alpha$, and peroxiredoxins (Prdx 1, 2 and 3) as well as enhanced expression of a variety genes, among them $N A D H$ ubiquinone oxidoreductase subunit 2, myosin light chain 1 ventricular isoform, fatty acid binding proteins, adipophilin, mitochondrial uncoupling protein 2, pyruvate dehydrogenase kinase 4, and RNA binding motif protein 3 (Fahlman et al., 2000; Hittel and Storey, 2001; Eddy et al., 2004; Brauch et al., 2005; Mamady and Storey, 2006; Morin and Storey, 2008; Yan et al., 2007). These proteins are responsible for making critical alterations that aid torpor survival such as enhancing capacity for fatty acid metabolism and contributing to long term cell preservation by elevating protein chaperones and antioxidant defenses. As well, previous studies have shown evidence of intensified protein synthesis during arousal from hibernation; the increase of mTORC1 
phosphorylation during the early arousal stages could support an enhanced protein synthesis as an aid to rapidly restoring euthermic metabolism and replacing proteins that were damaged during torpor before the animal descends into its next torpor bout (Zhegunov et al.,1988).

Selected synthesis of proteins that combat potential reperfusion damage due to the rapid increase in oxidative metabolism may also occur during arousal. One such example is nuclear factor-like $2(\mathrm{Nrf} 2)$, a transcription factor that up-regulates antioxidant defense proteins which increased by 1.5-fold during EA in cardiac muscle (Morin et al., 2008). This could suggest that the increase in mTORC1 phosphorylation during the heterothermic stages serves to induce expression of genes responsible for regulating the metabolic processes required for transition into and out of torpor.

\subsubsection{Regulation of mTORC1 downstream targets}

In addition to examining the $\mathrm{mTORC} 1$ complex and its upstream regulators, the state of the translational complex was also assessed by analyzing the phosphorylation states of direct downstream targets of mTORC1 including 4E-BP1, p70S6K, and S6 ribosomal protein. Both 4E-BP1 and p70S6K play crucial roles in the assembly of the active ribosomal translational complex. The translational repressor, 4E-BP1 in its hypophosphorylated form interacts with eIF4E and prevents it from binding with eukaryotic translation initiation factor 4 gamma (eIF4G) to form the active cap-dependent translational complex (Hay and Sonnenberg, 2004). By contrast, phosphorylation of 4E$\mathrm{BP} 1$ releases eIF4E to allow it to join the eIF4 complex. p70S6K is a downstream kinase that phosphorylates and activates the ribosomal S6 protein, a critical component of the 
40S ribosome subunit (Pestova and Hellen, 2003). It is well known that the phosphorylation status of S6 protein influences the rate of protein synthesis, an increase in S6 protein phosphorylation increasing the rate of translation initiation and elongation (Nielsen et al., 1982). FIG. 3.6 shows that 4E-BP1 is hypophosphorylated during torpor (both ET and LT) in skeletal muscle but in the early arousal stage, 4E-BP1 was hyperphosphorylated in both skeletal muscle and heart (FIG. 3.7). These data suggest that there would be increased eIF4E binding by 4E-BP1 during torpor that could potentially suppress translation; this is reversed again during arousal when 4E-BP1 becomes hyperphosphorylated. Similarly, 4E-BP1 was hypophosphorylated during torpor in liver of golden mantled-squirrels (van Breukelen et al., 2004). The pattern of change in p-4E-BP1 Thr36 was similar to that seen for p-mTOR Ser-2448, consistent with the known role of 4E-BP1 as a direct substrate of mTORC1. More importantly, this could implicate the mTORC1 complex in reversibly regulating the cap-dependent initiation of protein translation over the torpor-arousal cycle by regulating eIF4E availability.

mTOR is also known to regulate the translation of 5'TOP (terminal oligopyrimidine tract) mRNA, a small class of mRNA that encodes ribosomal proteins and translational complex proteins (Meyuhas et al., 1996). The translation of 5'TOP mRNA is promoted by mTOR via activation of the $40 \mathrm{~S}$ ribosomal protein $\mathrm{S} 6$ via $\mathrm{p} 70 \mathrm{~S} 6 \mathrm{~K}$ (Schmelzle and Hall, 2000). However, it should be noted that recent studies have suggested alternative signaling pathways that regulate the expression of 5'TOP mRNA upon mitogen stimulation (Pende et al., 2004). FIG. 3.6 and FIG. 3.7 show that phosphorylation of p70S6K at Thr-389 was significantly enhanced during EA in both skeletal and cardiac muscle. Accordingly, phosphorylation of S6 protein at Ser-235 was 
elevated in both muscles during EA as compared with the previous LT stage (2.2-fold in skeletal muscle, and 2.4-fold in cardiac muscle) and in heart as compared with EC. Interestingly, the phosphorylation pattern of p70S6K did not correlate with S6 protein in skeletal muscle and there was also a lack of correlation between the phosphorylation pattern of p70S6 $\mathrm{K}$ and the upstream regulators (TSC2, mTOR). It has previously been suggested that the regulation of p70S6K involves a complex hierarchy of phosphorylation events regulated by several kinases; phosphorylation at Thr-389 represents one of the early phosphorylation sites required for full p70S6K activation (Pullen and Thomas, 1997). However, the phosphorylation pattern of S6 protein at Ser-235 mimicked that of mTOR Ser-2448, where reduced phosphorylation was seen during torpor in skeletal muscle, and a strong increase was observed during early arousal in both muscles. Similar results were also reported previously for I. tridecemlineatus liver where the phosphorylation pattern of p70S6K at Thr-389 did not correlate with the pattern for S6 Ser235 (McMullen and Hallenbeck, 2010). Since mTOR does not phosphorylate S6 protein directly, a role for p70S6K is still implicated although activation of p70S6K might depend on phosphorylation sites not assessed in the present study. As well, it should also be noted that phosphorylation of S6 Ser-235 has previously been shown to be regulated via mTOR independent pathways such as mitogen activated protein kinases (Pende et al, 2004).

\subsubsection{Tissue specific mode of mTOR regulation}

This study also provides a comparative analysis on the differences in mTOR signaling between cardiac and skeletal muscles over torpor-arousal. Hibernators endure 
long periods of skeletal muscle disuse during torpor; a comparable period of disuse by human skeletal muscle results in a significant decrease in functional capacity and loss of muscle mass, also known as atrophy (Lee et al., 2008; Brooks et al., 2011). Previous studies have shown that golden-mantled ground squirrels (Spermophilus lateralis) experience some skeletal muscle atrophy during hibernation with a decrease in gastrocnemius mass of $21 \%$, and semitendinosus mass of $42 \%$; by contrast, cardiac muscle experiences ventricular hypertrophy with an increase of $21 \%$ tissue mass (Wickler et al., 1991).Since skeletal muscle cells are terminally differentiated, the development of muscle mass is regulated by the balance of anabolic and catabolic activities (Stitt et al., 2004). Studies have shown that activated Akt-mTOR signaling regulates loading of skeletal muscle cells by promoting protein synthesis; in addition, Akt actively inhibits the nuclear translocation of forkhead box O protein (FoxO), a transcription factor that promotes muscle proteolysis by regulating ubiquitin-proteasome pathways (Stitt et al., 2004; Sandri et al., 2004). In the present study, the mTOR signaling network in skeletal muscle was suppressed during torpor, suggesting a potential decrease in anabolic activities in skeletal muscle. The physiological consequence of reduced anabolic muscle growth via reduced protein synthesis would be skeletal muscle atrophy, as previously observed in S. lateralis during hibernation (Wickler et al., 1991; Nowell et al., 2011). Interestingly, however, Nowell et al. (2011) show that myosin remodeling and selective atrophy are early responses in the hibernating season and that atrophy is not progressive over the final three months of torpor. This suggests that although some degree of atrophy is allowed, there is resistance to a continued loss of muscle mass. Our findings fit with this idea by documenting the reactivation of the mTOR pathway during 
arousal which could suggest that renewed protein synthesis to restore needed proteins can recur each time that animals arouse. A similar pattern in $\mathrm{mTOR}$ regulation was also observed in hibernating bats, where a decrease in p-mTOR during hibernation was coupled with a biphasic oscillation of p-mTOR expression during the post-arousal torpor to re-arousal cycle (Lee et al., 2010).

One characteristic of ground squirrel hibernation is the development of a hypertrophic cardiac muscle during torpor. Although the majority of physiological functions are suppressed during hibernation, the heart continues to function, at a substantially reduced rate (a decrease from 200-300 beats per minute in euthermia to 3-5 bpm in torpor) but requiring increased power output (Carey et al., 2003; Zatzman, 1984). The present study shows that the mTOR signaling pathway in heart was largely maintained in its euthermic pattern during torpor, suggesting that preservation of the potential for active protein synthesis is needed to support a hypertrophic condition and sustain cardiac work during torpor. The observed increase in cardiac mTOR signaling during arousal again suggests, as in skeletal muscle, an increase in translational capacity to support the rapid increase in heart rate during arousal, restore proteins that were potentially damaged during the torpor phase, and enhance cellular defenses to minimize reperfusion damage (Morin et al., 2008; Yan et al., 2008).

\subsection{CONCLUSION}

In conclusion, the present chapter examined the regulation of the mTOR signaling network and demonstrated that the mTORC1 complex is differentially regulated during hibernation in skeletal and cardiac muscle. Our data show that depression of the mTOR 
signaling pathway occurs during torpor in skeletal muscle and suggests that the regulation of mTOR plays a major role in reducing cap-dependent protein synthesis. The current data all support a state of translational depression during torpor (ET, LT) in skeletal muscle. The phosphorylation status of 4E-BP1 and S6 protein, the responses of members of the mTORC1 complex (mTOR, PRAS40, G $\beta \mathrm{L}$ ) and the changes in mTORC1 upstream regulators (TSC2, Akt) are all consistent with inhibition of protein synthesis during torpor. By contrast, cardiac muscle maintained mTOR signaling capacity at neareuthermic levels throughout torpor. Both skeletal and cardiac muscle show evidence of periods of enhanced mTOR signaling - during entrance into torpor and during arousal from torpor in the skeletal muscle, and during arousal from torpor in the cardiac muscle supporting the idea that new protein synthesis is needed at both of these times. In particular, coordinated changes in mTOR signaling components indicate a strong enhancement of protein synthesis capacity during arousal. However, the mechanisms for enhanced mTOR signaling appear somewhat different between the two tissues based on the regulation of mTORC1 interacting proteins (PRAS40, G $\beta \mathrm{L}$ ). The differential regulation of translational capacity in skeletal versus cardiac muscle may underlie the physiological consequences for these muscles over hibernation cycles - the trend to atrophy in skeletal muscle and hypertrophy in cardiac muscle. More importantly, the reversible nature of mTOR signaling demonstrates the unique molecular plasticity exhibited by thirteen-lined ground squirrels during hibernation. 


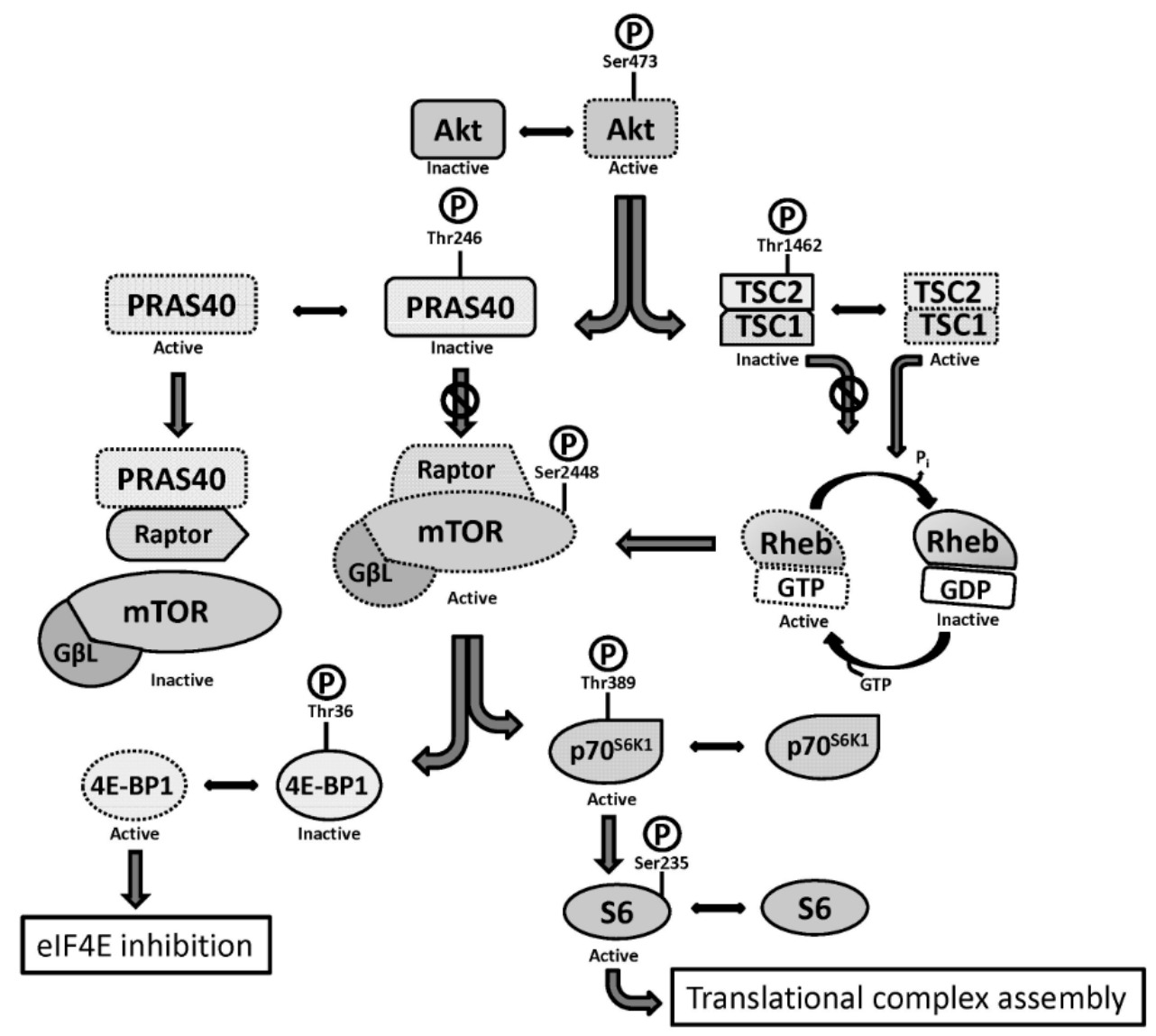

\section{FIG. 3.1 The mTORC1 signaling pathway.}

Akt influences mTORC1 activation through its phosphorylation of TSC2 and PRAS40. Akt mediated TSC2 phosphorylation inhibits the conversion of Rheb:GTP $\rightarrow$ Rheb:GDP, preventing the auto-phosphorylation of mTORC1. Meanwhile, Akt mediated phosphorylation of PRAS40 prevents access by mTORC1 to its downstream substrates (4E-BP1, p70S6K) through its interaction with RAPTOR. The inability of mTORC1 to phosphorylate its downstream substrates 4E-BP1 and p70S6K can arrest formation of the active translational complex (Hay and Sonenberg, 2004). 

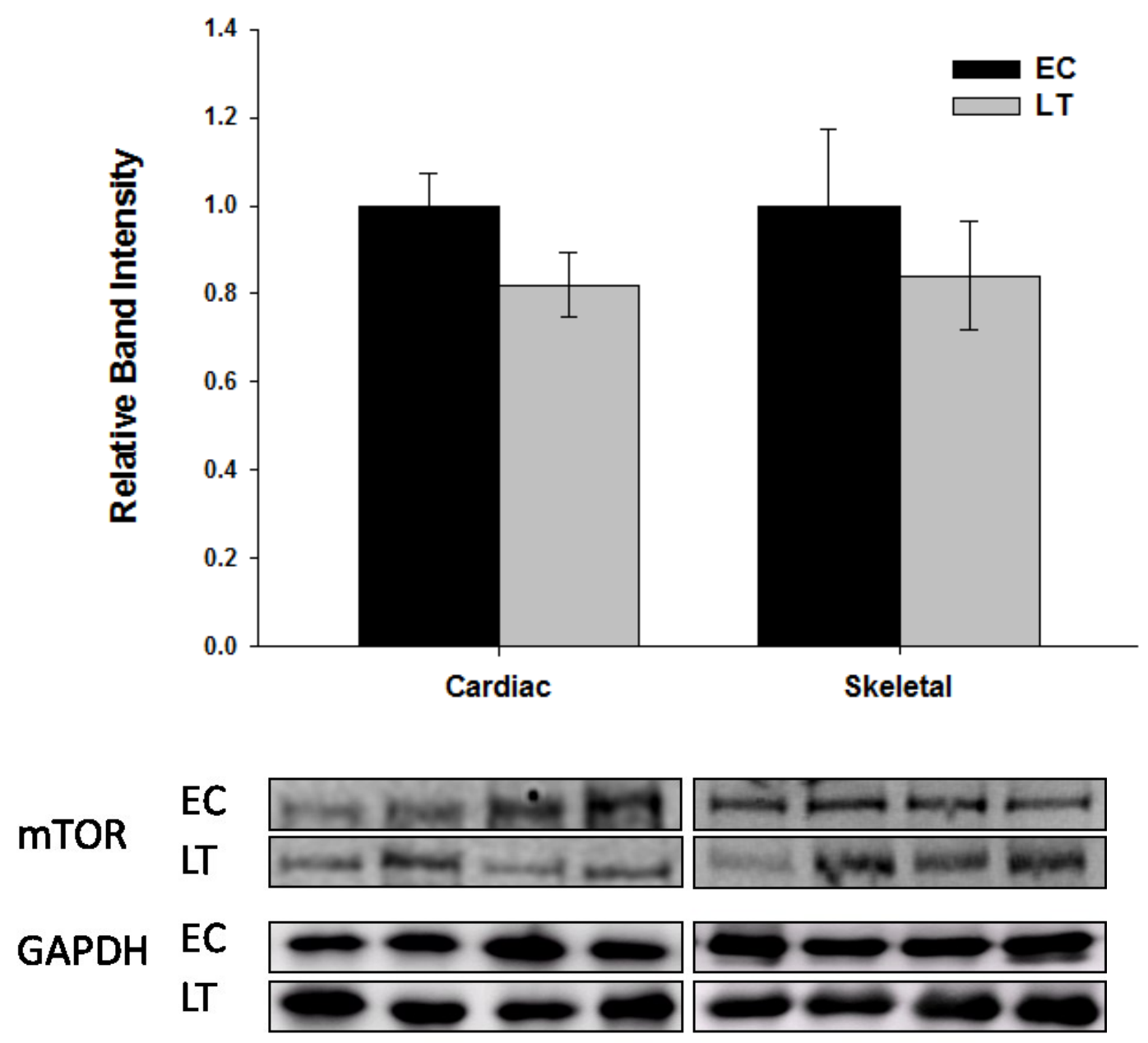

FIG. 3.2. Regulation pattern of $m$ TOR protein in cardiac and skeletal muscle of $I$. tridecemlineatus as determined by Western immunoblotting.

Relative expression of mTOR and housekeeping GAPDH protein over the torporarousal cycle in the liver of 13-lined ground squirrels. Western blot bands of mTOR were detected at $\sim 290 \mathrm{kDa}$ and the housekeeping protein GAPDH was detected at $\sim 32 \mathrm{kDa}$. Data are means \pm S.E.M., $n=4-5$ independent trials on tissues from different animals. EC, Euthermic control; LT, late torpor. 


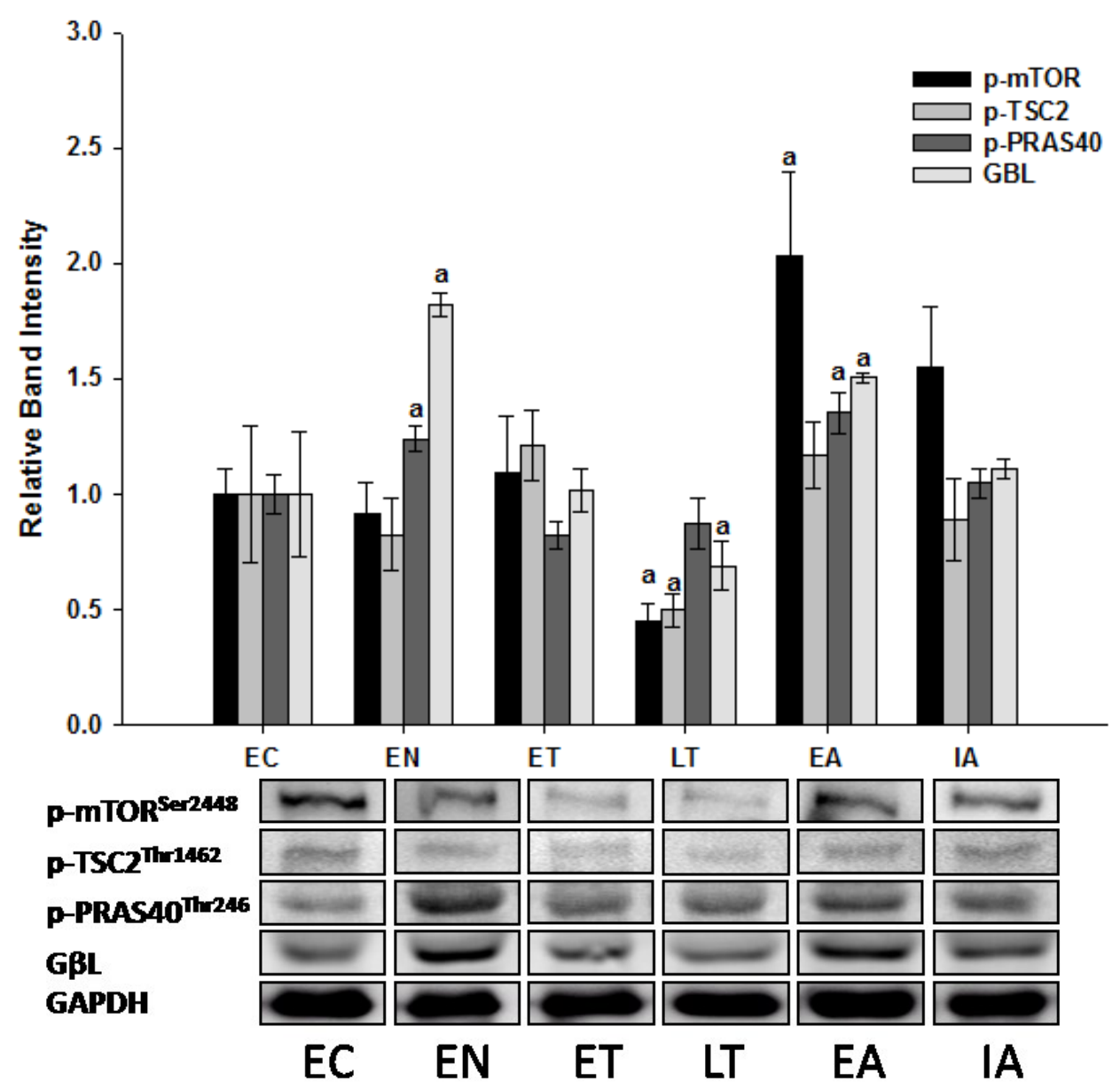

FIG. 3.3. Regulation pattern of mTOR regulator proteins in skeletal muscle of $I$. tridecemlineatus as determined by Western immunoblotting.

Relative expression of p-mTOR (Ser-2448), p-TSC2 (Thr-1462), p-PRAS40 (Thr246), G $\beta \mathrm{L}$, and GAPDH protein over the torpor-arousal cycle in liver of 13-lined ground squirrels. Representative Western blot bands of p-mTOR were detected at $\sim 290 \mathrm{kDa}, \mathrm{p}$ TSC2 at $\sim 150 \mathrm{kDa}, \mathrm{p}-\mathrm{PRAS} 40$ at $\sim 40 \mathrm{kDa}, \mathrm{G} \beta \mathrm{L}$ at $\sim 38 \mathrm{kDa}$, and housekeeping protein GAPDH was detected at $\sim 32 \mathrm{kDa}$. Data are means \pm S.E.M., $\mathrm{n}=4-5$ independent trials on tissues from different animals. EC, euthermic control; EN, early entrance into torpor; ET, early torpor; LT, late torpor; EA, early arousal; IA, interbout arousal. a-Significantly different from corresponding EC values ( $\mathrm{P}<0.05$, ANOVA post hoc Holm-Sidak test). 

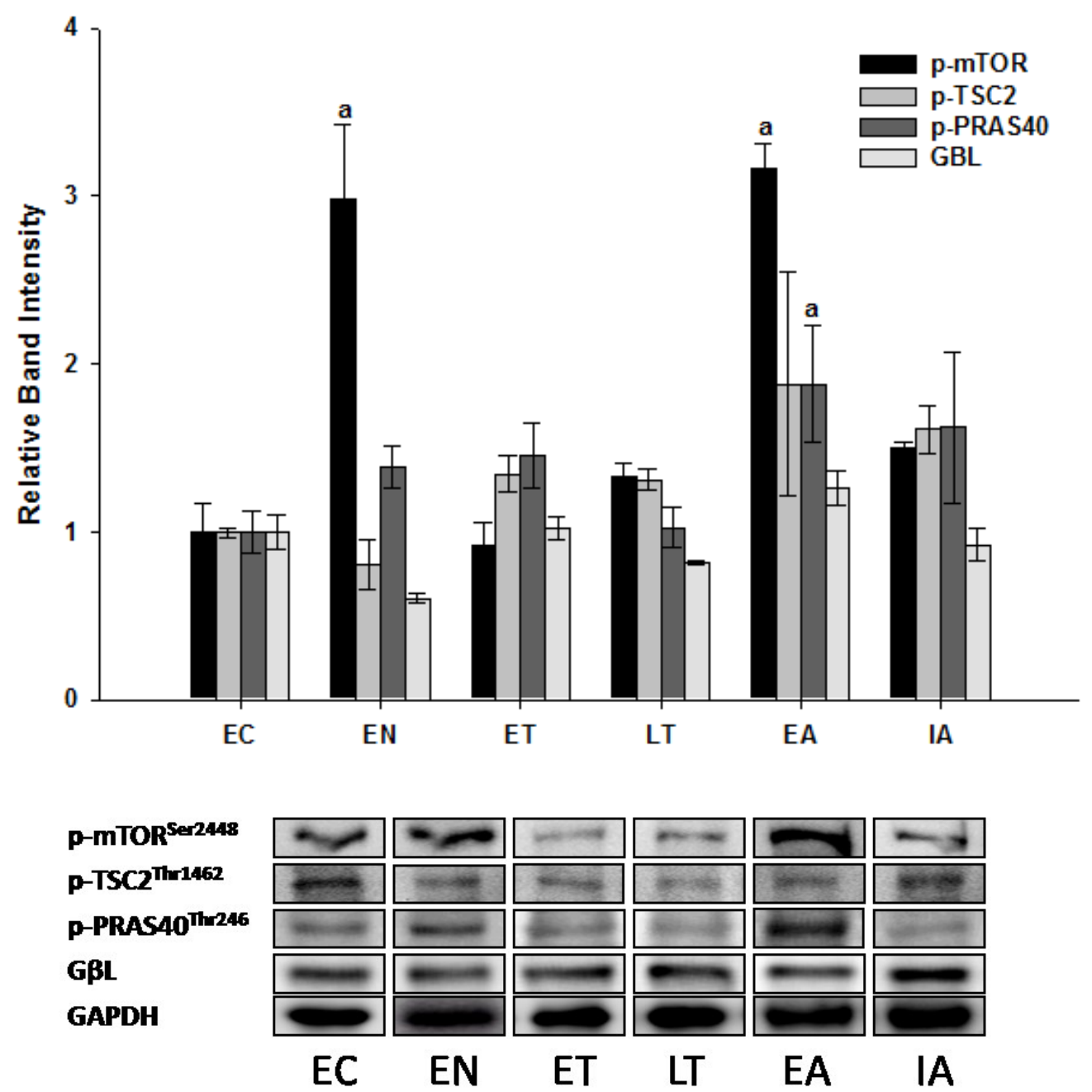

FIG. 3.4. Regulation pattern of mTOR regulator proteins in cardiac muscle of $I$. tridecemlineatus as determined by Western immunoblotting.

Relative expression of p-mTOR (Ser-2448), p-TSC2 (Thr-1462), p-PRAS40 (Thr246), G $\beta \mathrm{L}$, and GAPDH protein over the torpor-arousal cycle in liver of 13-lined ground squirrels. Representative Western blot bands of p-mTOR were detected at $\sim 290 \mathrm{kDa}$, pTSC2 at $\sim 150 \mathrm{kDa}, \mathrm{p}-\mathrm{PRAS} 40$ at $\sim 40 \mathrm{kDa}, \mathrm{G} \beta \mathrm{L}$ at $\sim 38 \mathrm{kDa}$, and GAPDH at $\sim 32 \mathrm{kDa}$. a-Significantly different from corresponding EC values $(\mathrm{P}<0.05$, ANOVA post hoc Holm-Sidak test). 


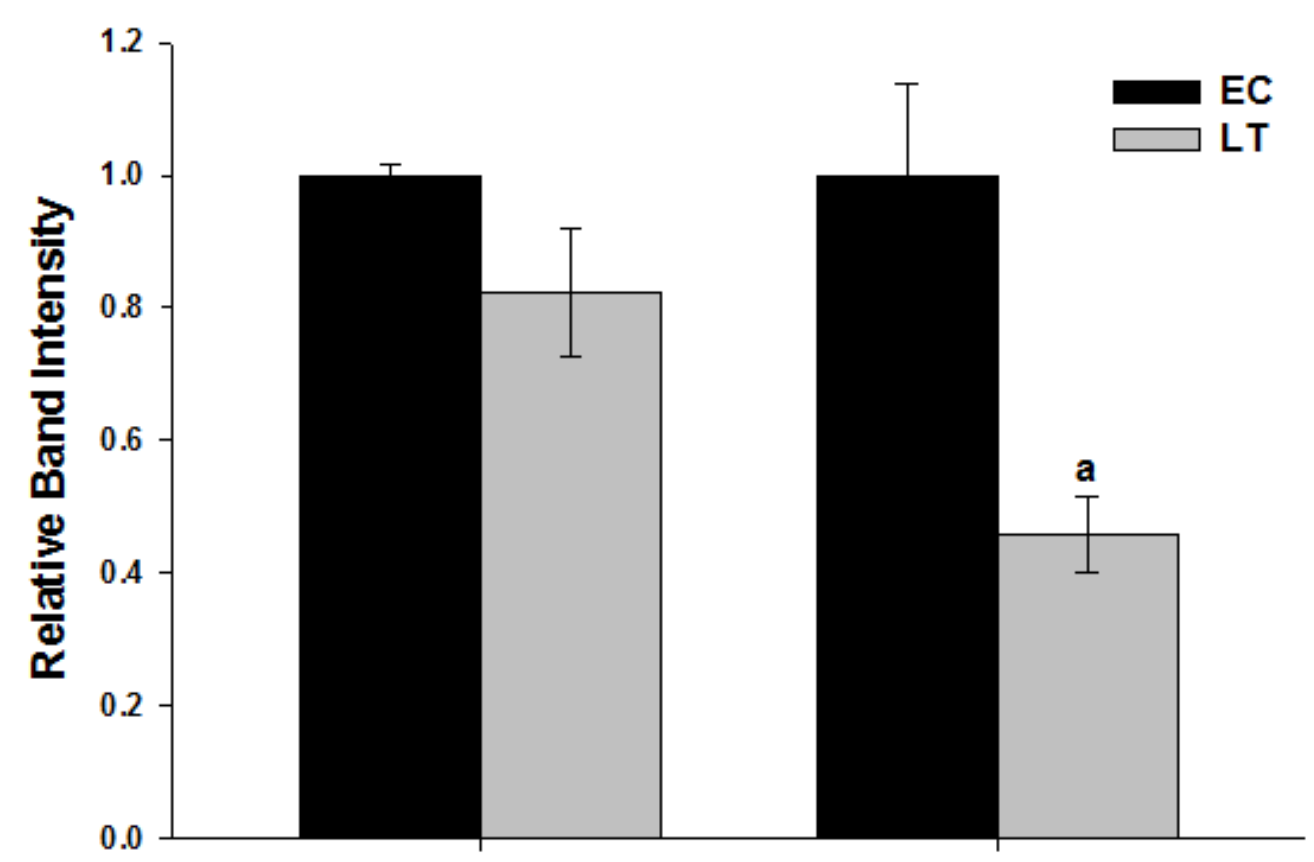

Cardiac

Skeletal

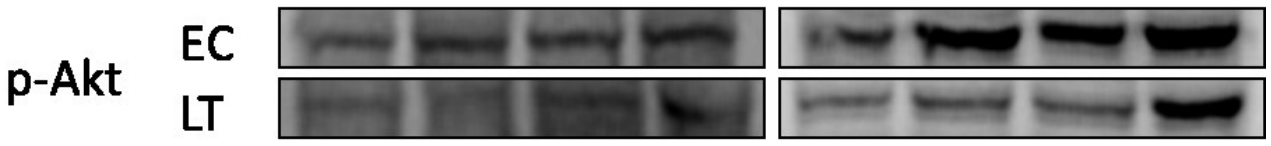

FIG. 3.5. Regulation pattern of phosphorylated Akt protein in cardiac and skeletal muscle of I. tridecemlineatus as determined by Western immunoblotting.

Relative expression of mTOR and the housekeeping GAPDH protein over the torpor-arousal cycle in liver of 13-lined ground squirrels. Western blot bands of mTOR were detected at $\sim 290 \mathrm{kDa}$ and housekeeping protein GAPDH was detected at $\sim 32 \mathrm{kDa}$. Data are means \pm S.E.M., $n=4-5$ independent trials on tissues from different animals. EC, euthermic control; LT, late torpor. a-Significantly different from corresponding EC values $(\mathrm{P}<0.05$, ANOVA post hoc Holm-Sidak test). 

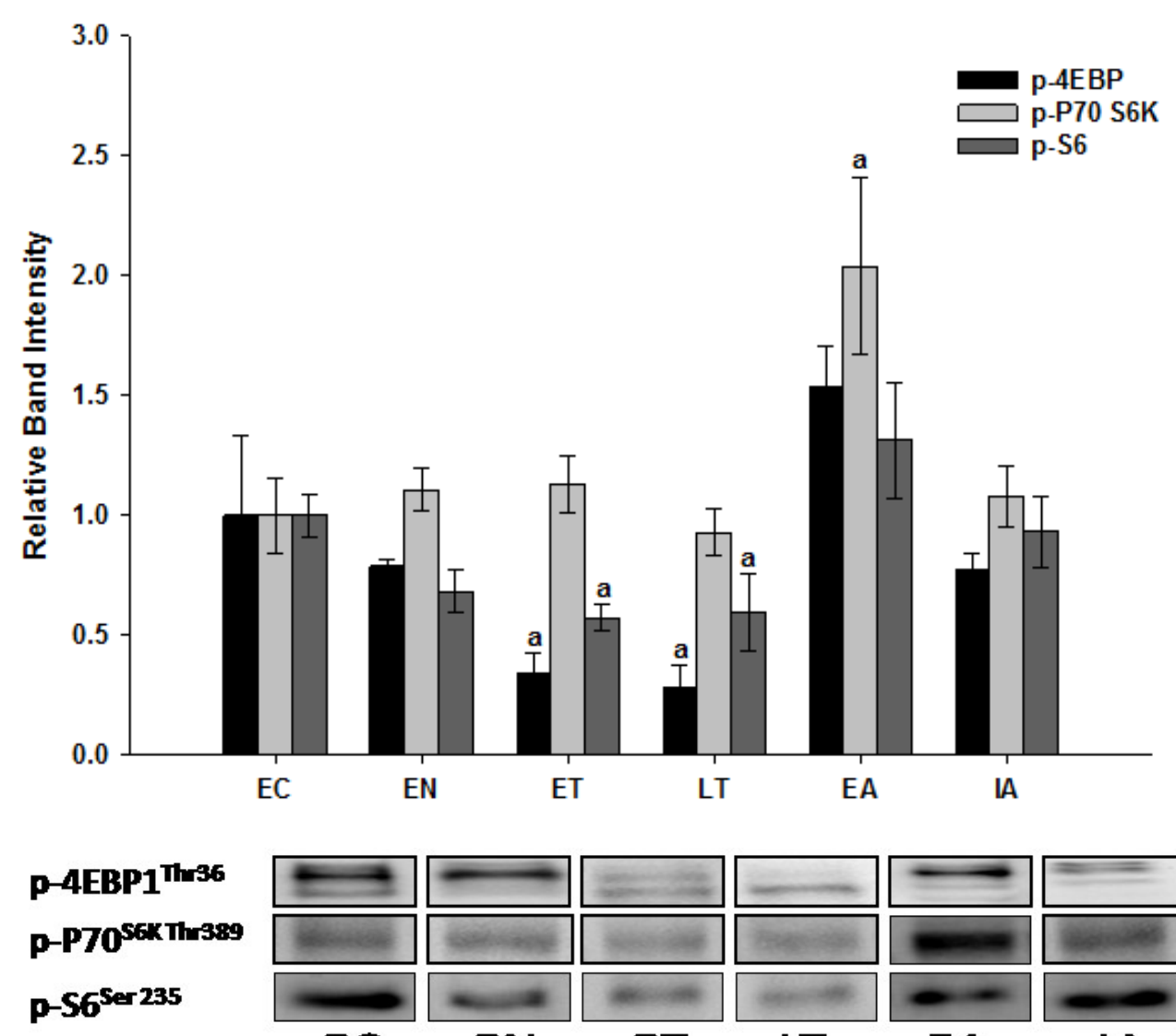

EC

EN

ET
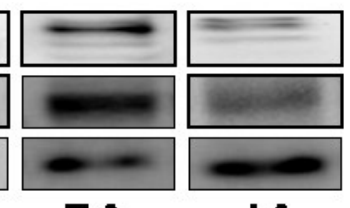

EA IA

FIG. 3.6. Regulation pattern of mTOR downstream proteins in skeletal muscle of $I$. tridecemlineatus as determined by Western immunoblotting.

Relative expression of p-4EBP1 (Thr-36), p-p70S6K (Thr-389), and p-S6 (Ser235) over the torpor-arousal cycle in liver of 13-lined ground squirrels. Representative Western blot bands of p-4EBP1 were detected at $\sim 15 \mathrm{kDa}, \mathrm{p}-\mathrm{p} 70 \mathrm{~S} 6 \mathrm{~K}$ at $\sim 70 \mathrm{kDa}$, and p$\mathrm{S} 6$ at $\sim 32 \mathrm{kDa}$. Data are means \pm S.E.M., $\mathrm{n}=4-5$ independent trials on tissues from different animals. a-Significantly different from corresponding EC values $(\mathrm{P}<0.05$, ANOVA post hoc Holm-Sidak test). 

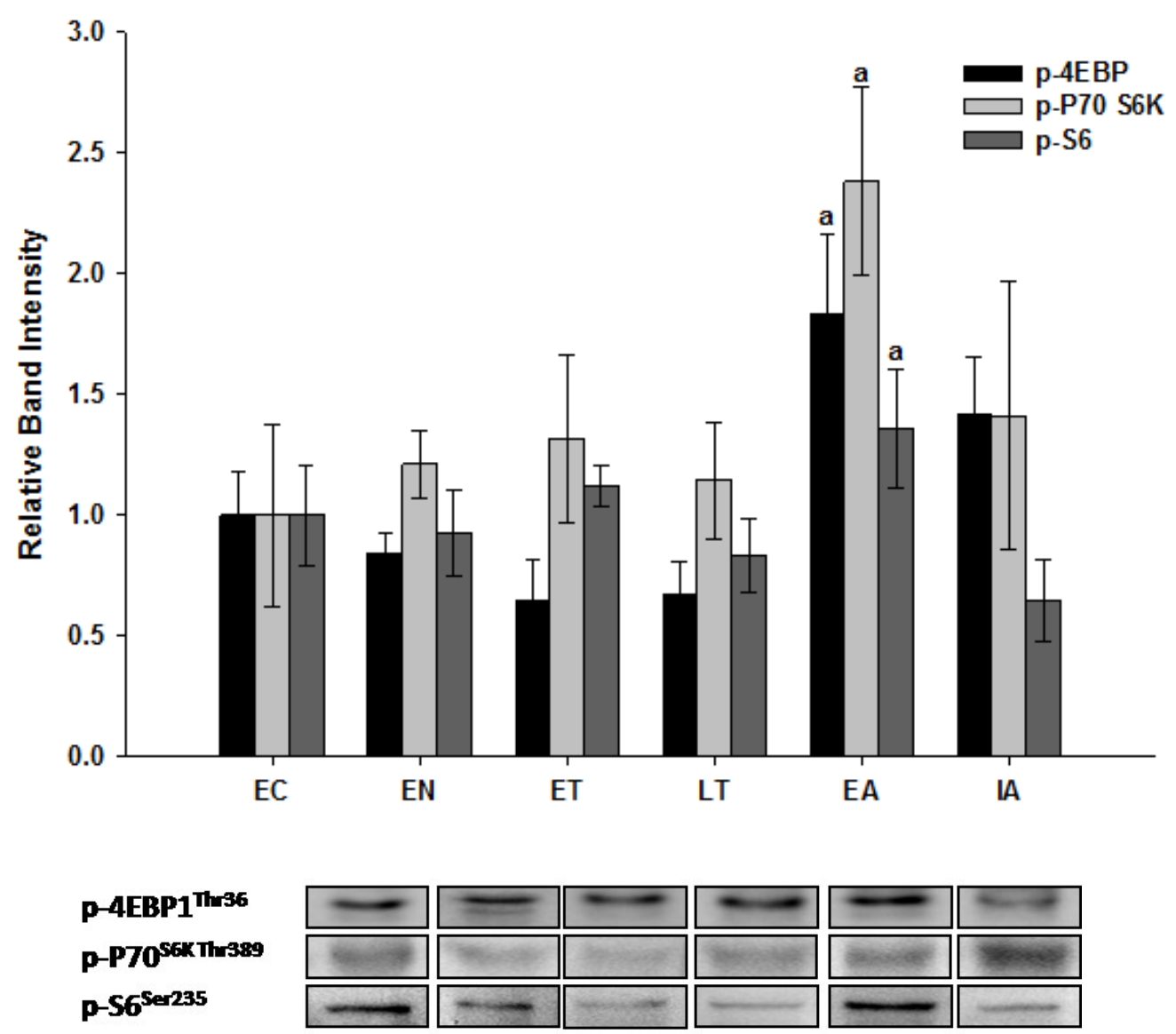

FIG. 3.7. Regulation pattern of mTOR downstream proteins in cardiac muscle of $I$. tridecemlineatus as determined by Western immunoblotting.

Relative expression of p-4EBP1 (Thr-36), p-p70S6K (Thr-389), and p-S6 (Ser235) over the torpor-arousal cycle in liver of 13-lined ground squirrels. Representative Western blot bands of p-4EBP1 were detected at $\sim 15 \mathrm{kDa}, \mathrm{p}-\mathrm{p} 70 \mathrm{~S} 6 \mathrm{~K}$ at $\sim 70 \mathrm{kDa}$, and p$\mathrm{S} 6$ at $\sim 32 \mathrm{kDa}$. Data are means \pm S.E.M., $\mathrm{n}=4-5$ independent trials on tissues from different animals. a-Significantly different from corresponding EC values $(\mathrm{P}<0.05$, ANOVA post hoc Holm-Sidak test). 


\section{CHAPTER 4}

\section{POST-TRANSLATIONAL REGULATION OF PTEN PHOSPHATASE DURING TORPOR}




\subsection{INTRODUCTION}

The regulation of metabolism is often controlled by crucial rate-limiting proteins, whose functionality can dictate the 'on' or 'off' status of specific cellular processes. As previously discussed, one key mechanism for such regulation is reversible protein phosphorylation, which controls the functionality of many cellular proteins, in processes including carbohydrate metabolism, protein synthesis, gene transcription, apoptosis, the cell cycle, and many others (Brooks and Storey, 1992; Chen et al., 2001; Morin and Storey, 2006; Rouble et al., 2013). The reversible phosphorylation of proteins is catalyzed by the actions of protein kinases and protein phosphatases, working as antagonistic regulators to modify the phosphorylation status of a given target (Cohen, 2002; Wu et al., 2013). Although the majority of phosphatases target protein molecules as their primary substrates, phosphatases such as PTEN (phosphatase and tensin homologue deleted on chromosome 10) display intrinsic activity towards both protein and lipid derivatives (Zhang et al., 2012). PTEN was initially identified as a tumour suppressive gene that was found to be deleted in multiple sporadic tumours, most prevalently targeted by germline mutations such as Cowden disease (Li et al., 1997; Steck et al., 1997). The primary catalytic function of PTEN is the dephosphorylation of phosphatidylinositol $(3,4,5)$-trisphosphate $\left(\mathrm{PIP}_{3}\right)$, a second messenger lipid that activates 3-phosphoinositide dependent protein kinase-1 (PDK-1), and subsequently Akt, in the insulin signaling pathway (Maehama and Dixon, 1998). Consequently, active PTEN functions as a negative regulator of the insulin pathway, and suppresses cell growth and proliferation signals stimulated through Akt by regulating the abundance of $\mathrm{PIP}_{3}$. 
The protein structure of PTEN is strongly conserved across species, and is composed of five functional domains: PBD (phosphatidylinositol-4,5-bisphosphate [PtdIns(4,5) $\left.\mathrm{P}_{2}\right]$-binding domain), phosphatase domain, $\mathrm{C} 2$ domain, C-terminal tail domain, and a PDZ domain (Song et al., 2012). The C-terminal tail domain of PTEN is regulated by phosphorylation, which functions to control both the catalytic activity and structural stability of the protein (Vazquez et al., 2000; Rabinovsky et al., 2009). Phosphorylation at multiple C-terminal residues, including Ser-380, Thr-382, Thr-383, and Ser-385 have been shown to decrease PTEN phosphatase activity, while promoting structural stability. Conversely, dephosphorylation of these residues has been shown to increase PTEN activity, while decreasing stability. In addition to post-translational regulation, PTEN is also regulated at the transcriptional level by non-coding RNAs. In malignancies such as leukaemia and Cowden disease, PTEN expression is downregulated by the actions of miR-19 and miR-21, that target PTEN mRNA transcripts (Pezzolesi et al., 2008; Bai et al., 2011).

Although its primary role is in the insulin signaling pathway, PTEN functions to regulate aspects of energy metabolism, by coordinating metabolic signals involved in cell growth (Song et al., 2021). Accordingly, PTEN can be considered to be a major regulator of metabolic reprogramming, a process by which metabolic functions are altered based on changes in the cellular environment. This is best characterized in cancer cells, where the actions of proto-oncogenes and tumour suppressors assist in the activation of cell proliferation by reprogramming cellular metabolism (Song et al., 2012, Ward and Thompson, 2012). This concept of metabolic reprogramming can also be applied to different forms of hypometabolism; for example, generation and utilization of metabolic 
fuels are altered through actions of different effector molecules in response to environmental changes. Mammalian hibernation clearly involves such metabolic reprogramming. Major changes in metabolic fuel use occur (a switch to a primary reliance on lipid metabolism from carbohydrate metabolism) and energy reprioritization (major suppression of protein synthesis, active ion pumps, and gene transcription) is observed during periods of hypometabolism (Buck et al., 2001; Frerichs et al., 1998; Morin and Storey, 2006; MacDonald and Storey, 1999). In Caenorhabditis elegans, a mutation that removed the phosphatase catalytic domain of the PTEN homologue, Daf18 , inhibited the induction of a dauer phenotype when worms were shifted towards a higher habitat temperature (previously shown to induce dauer in Daf-18 wild-types) (Mihaylova et al., 1999). This suggests that PTEN is a crucial regulator of metabolic rate depression, and the catalytic activity of PTEN is required to balance the levels of $\mathrm{PIP}_{3}$, to regulate signal transduction in the insulin pathway.

Other chapters in this thesis show that components downstream of the insulin signaling pathway including Akt, and the mTORC1 complex are selectively supressed in skeletal muscle during torpor (see Chapter 3). The present chapter characterizes the enzymatic regulation of PTEN during torpor, along its regulation patterns at the transcriptional and translational level. It is hypothesized that PTEN will be activated during torpor to promote suppression of the insulin signaling pathway, and that this activation is likely regulated by the mechanism of C-terminal tail phosphorylation. 


\subsection{RESULTS}

\subsubsection{Phosphorylation pattern of phosphatase and tensin homolog (PTEN)}

The regulation of PTEN protein was characterized in skeletal muscle at the protein level via Western immunoblotting. Three different parameters of PTEN were analyzed using three specific antibodies - one antibody that quantifies total PTEN protein, one that is specific for phosphorylated residues on PTEN within the C-terminal peptide that contains Ser-380/ Thr-382/ Thr-383, and one that is specific for this same peptide in its non-phosphorylated state. As seen in FIG. 4.1, levels of phosphorylated PTEN when expressed as a ratio to total PTEN, were significantly decreased in EN and IA by $52 \%$ and $31 \%$, respectively, compared to $\mathrm{EC}(\mathrm{P}<0.05)$. Using antibodies that specifically detect Ser-380/ Thr-382/ Thr-383 residues in the non-phosphorylated state, it was shown that levels of non-phosphorylated PTEN when expressed as a ratio to total PTEN, was significantly decreased during EN by $28 \%$ and increased by 1.44 -fold during LT when compared to $\mathrm{EC}(\mathrm{P}<0.05)$.

\subsubsection{Phosphorylation pattern of 3-phosphoinositide dependent protein kinase-1} $(P D K-1)$

The phosphorylation state of PDK-1, a downstream kinase of PTEN was also characterized using Western immunoblotting in the skeletal muscle. As seen in FIG. 4.2., the relative ratio of PDK-1 phosphorylated at Ser-241 was significantly decreased by $32 \%$ during LT compared to EC $(\mathrm{P}<0.05)$; meanwhile, this ratio was stably maintained for the other time points of the torpor-arousal cycle. 


\subsubsection{Transcriptional regulation of PTEN and PDK-1 mRNA expression}

To determine if PTEN and PDK-1 are differentially regulated at the transcriptional level in the skeletal muscle during the torpor-arousal stage, RT-PCR was used to measure the relative mRNA transcript levels of both genes. Shown in FIG. 4.3., the relative levels of PTEN mRNA transcripts remained constant in all six stages of the torpor arousal cycle. Similarly, mRNA expression of $P D K-1$ also remained unchanged throughout the time course, with no significant increases or decreases observed in the skeletal muscle. This suggests that both PTEN and PDK-1 are not regulated at the transcriptional level in the skeletal muscle of ground squirrels during hibernation.

\subsection{5 cDNA cloning of partial squirrel PTEN transcript}

The PCR analysis of PTEN yielded an amplicon of 321 bp in length (FIG. 4.4). The nucleotide sequence of the amplicon was determined, closely matched the corresponding part of the open reading frame of PTEN of other mammals and translated into a sequence of 100 amino acids. When compared to other mammals, as shown in FIG. 4.5., the homology tree showed a high degree of conservation between the squirrel sequence and the mouse (99.3\%), rat (98.9\%), and human (98.9\%) PTEN sequences.

\subsection{6 cDNA cloning of partial squirrel PDK-1 transcript}

The PCR analysis of $P D K-1$ yielded an amplicon of 426 bp in length (FIG. 4.6). The nucleotide sequence of the amplicon closely matched the open reading frame of other mammals and translated into a 141 amino acid sequence. When compared to other mammals, as shown in FIG. 4.7., the homology tree produced high degree of 
conservation between the squirrel sequence to mouse $(96.2 \%)$, rat $(96.8 \%)$, and human $(92.4 \%)$

\subsubsection{Phosphatase activity of PTEN}

To determine if increased ratio of non-phosphorylated PTEN to total PTEN in late torpor affected the enzymatic activity of PTEN, phosphatase activity and kinetic parameters were measured for PTEN isolated from EC and LT stages using soluble $\mathrm{PIP}_{3}$ lipid secondary messenger as the substrate. FIG. 4.8 shows that PTEN displayed Michaelis-Menten kinetics with hyperbolic substrate saturation curves observed for the enzyme from both EC and LT stages. Maximal activity was observed with at $\mathrm{PIP}_{3}$ substrate concentrations in the range of $20-25 \mu \mathrm{M}$. As seen in Table 4.1, the Km of $\mathrm{PIP}_{3}$ was significantly lower during LT at $3.96 \pm 0.47 \mu \mathrm{M}$ as compared to the EC value of $8.96 \pm 1.17 \mu \mathrm{M}(\mathrm{P}<0.05)$, indicating an increased affinity of PTEN for $\mathrm{PIP}_{3}$ as a substrate during hibernation. The activity of PTEN was normalized to the expression of total PTEN present in each experimental condition, as determined by Western immunoblotting of the same sample PTEN activity was measured from. The $\mathrm{V}_{\max }$ measurement however showed no significant differences between euthermic control and late torpor, with values of 0.49 \pm 0.04 vs. $0.42 \pm 0.01 \mathrm{nmol} \mathrm{Pi} / \mathrm{min} / \mathrm{PTEN}$ respectively.

\subsubsection{Purification of PTEN phosphatase}

PTEN was partially purified from crude homogenates in order to characterize the structural stability of the phosphatase. FIG. 4.9 shows the representative protein elution profile for crude skeletal muscle supernatant (euthermic control) applied to a Heparin affinity chromatography and eluted with a $0-2 \mathrm{M}$ linear $\mathrm{NaCl}$ gradient. The protein 
elution profile, as assessed via the Bradford protein assay, showed a single broad peak of protein eluted over five fractions; the peak fractions 13-17 showed protein concentrations of $0.52,0.18,0.19,0.15$, and $0.09 \mathrm{mg} / \mathrm{mL}$ respectively. To determine the elution profile of PTEN phosphatase, a Western immunoblot was performed. As seen in FIG. 4.9., PTEN protein was observed in the initial wash fractions of $1-5$, and in $\mathrm{NaCl}$ eluted fractions of 13-19, with highest amount of PTEN observed in fraction 14. This shows that the peak observed in the protein elution profile corresponds to the elution of PTEN protein. Peak fractions were pooled and used for subsequent experiments on enzyme structural stability; PTEN from both EC and LT stages was purified in this manner.

\subsubsection{Pulse-proteolysis analysis of PTEN structural integrity.}

To determine if changes in phosphorylation content of PTEN during late hibernation had an effect on the structure stability of the protein, the sensitivity of partially purified PTEN from EC versus LT stages to urea denaturation was assessed via the pulse-proteolysis technique. PTEN was incubated with varying concentrations of urea and then denatured PTEN was hydrolyzed by 10 minute incubation with thermolysin. Subsequent electrophoresis of fractions and silver staining revealed the amount of native protein remaining at the $54 \mathrm{kDa}$ band that corresponds to PTEN (as verified by Western blotting). FIG. 4.10 shows that the percentage of native folded PTEN remaining decreased as a function of increasing urea concentration. Maximal PTEN unfolding was observed at urea concentrations of $3 \mathrm{M}$ and above where $20 \%$ detectable PTEN remained. The concentration of urea that caused half-maximal unfolding $\left(\mathrm{K}_{\mathrm{ND}}\right)$ was calculated from the data in FIG 4.10. $\mathrm{K}_{\mathrm{ND}}$ values for urea were $1.29 \pm 0.06 \mathrm{M}$ for $\mathrm{LT}$, 
significantly lower than the EC value of $1.62 \pm 0.05(\mathrm{P}<0.05)$ (Table 4.1). This suggests that PTEN from muscle of hibernating squirrels has a lower structural stability as compared to the euthermic control enzyme.

4.2.9 Proline, Glutamic acid, Serine, and Threonine (PEST) motif analysis of the PTEN protein sequence.

The fullength protein sequence of PTEN was retrived from ensemble database (ENSSTOT00000028610). Using the bioinformatics tool epestfind, potential sites of proteolytic cleavage in the PTEN amino acid sequence were identified using a PEST motif scan. Shown in FIG. 4.11, two potential PEST motifs are predicted near the Cterminus of PTEN, in the amino acid regions of 347 - 374 and 375 - 395. Interestingly, the second region of PEST motif contains the three key phosphorylation sites examined in this study, Ser-380, Thr-382, and Thr-383. This suggests that these three phosphorylation sites could potentially play a role in regulating structure integrity of PTEN phosphatase.

\subsection{DISCUSSION}

Second messengers function to relay signals from extracellular cues to biological responses; they include a variety of chemical such as cyclic nucleotides (e.g. cAMP, cGMP), calcium ions, and various lipid derivatives such as phosphatidyl inositol (Hirsch et al., 2007). The phosphorylation of phosphoinositides is governed by the actions of phosphoinositide 3-kinases (PI3-K) and PTEN, which regulate the relative abundance of $\mathrm{PIP}_{3}$ and $\mathrm{PIP}_{2}$. $\mathrm{PIP}_{3}$ binds to and triggers the activation of PDK-1, resulting in PDK-1 
phosphorylation of its downstream target Akt at Thr-308. This phosphorylation of Akt as well as an additional phosphorylation of Akt at Ser-473 activates the kinase activity of Akt (Sarbassov et al., 2005). Akt has been shown to target at least 75 different downstream targets, functioning to regulate a wide range of biological processes (Hirsch et al., 2007). The upstream regulation control of Akt through PTEN suggests that, by controlling the levels of the lipid second messenger $\mathrm{PIP}_{3}, \mathrm{PTEN}$ functions to trigger and regulate a wide range of cellular responses in metabolism, cell survival, and proliferation.

Previous studies have shown that the PTEN gene is a transcriptional target of activating transcription factor 2 (ATF-2), and both PTEN and ATF-2 show hypoxia induced upregulation in cardiomyocytes (Qian et al., 2012). As well, PTEN overexpression have been shown to inhibit cell proliferation, through reducing the expression of insulin growth factor-1 receptor and increased starvation induced apoptosis (Qian et al., 2012; Zhao et al., 2004). Conversely, during periods of excessive growth such as tumour proliferation, loss of PTEN activity along with decreased PTEN protein expression is widely observed (Ortega-Molina and Serrano, 2012). Based on previous studies on effects of PTEN functionality in metabolic regulation, it could be suggested that an increase in PTEN overall function would contribute to a state of metabolic depression, where a general suppression of Akt kinase activity is observed. The present chapter examined the enzymatic regulation of PTEN, along with protein and gene expression levels of PTEN and its downstream target PDK-1. The data show that PTEN is posttranslationally regulated via protein phosphorylation at its C-terminal tail residues, which functions to regulate both phosphatase activity and stability. 


\subsubsection{Post-translational and transcriptional regulation of PTEN and PDK-1}

The phosphorylation of PTEN has previously been shown to be a critical regulator of this enzyme's phosphatase activity, where phosphorylation of PTEN via caesin kinase 2 results in reduced catalytic activity along with increased structural stability (Torres and Pulido, 2001; Vazquez et al., 2000). To accurately assess the phosphorylation state of PTEN, the relative amounts of phosphorylated and non-phosphorylated PTEN were detected through Western blotting (FIG. 4.1), and expressed as a ratio to the total amount of PTEN. The phosphorylated content of PTEN was significantly reduced during EN and IA stages of hibernation, suggesting a reduced state of PTEN activity at these times; however, during the LT stage, a significant elevation of non-phosphorylated PTEN content was detected, suggesting a potential increase in PTEN activity. The decrease in PTEN phosphorylation during LT correlated with a decrease in PDK-1 phosphorylation at Ser-241 (FIG 4.2), a residue that has previously been shown to be associated with $\mathrm{PIP}_{3}$ mediated PDK-1 activation (Casamayor et al., 1999; Chan et al., 1999). This decreased phosphorylation of PDK-1 could be due to a decreased levels of $\mathrm{PIP}_{3}$, suggesting that PTEN is catalytically activated during torpor.

Interestingly, the mRNA expression of both $P T E N$ and $P D K-1$ were stable across the torpor-arousal cycle. This lack of transcriptional regulation could suggest that miRNA plays a minor role in PTEN regulation during torpor, and that primary regulatory control on both proteins may be at the posttranslational level. Posttranslational modifications of proteins have previously been shown to be crucial regulatory mechanisms for many enzymes. For example, major regulatory proteins such as Akt, mTOR, and PP2A are 
ubiquitously expressed over the torpor-arousal cycle, and are primarily regulated via post-translational modifications such as reversible protein phosphorylation (Chapter 3; Wu et al., 2013). Based on the sequence derived from PCR amplicons, the amino acid composition of portions of both PTEN and PDK-1 were predicted (FIG. 4.4 and 4.6), and aligned with the sequences from other mammalian species. Shown in FIG. 4.5, the amino acid sequence of squirrel PTEN exhibited a remarkable degree of conservation, with near perfect homology between human, mouse, rat and squirrel sequences. Similarly, the sequence of PDK-1 (FIG. 4.7) also showed a high degree of amino acid conservation compared with the other mammals. This high degree of similarity between amino acid sequences suggests that both PTEN and PDK-1 would have similar structures and functions as those described in other mammalian systems.

\subsubsection{Enzymatic regulation of PTEN}

Based on the phosphorylation pattern of PTEN observed in FIG 4.1, it was predicted that PTEN could show substantial differences in phosphatase activity and/or kinetic properties between EC and LT states. Hence, PTEN activity towards $\mathrm{PIP}_{3}$ substrate was measured for the enzyme from these two states to determine the functional relationship between phosphorylation and activity. Shown in FIG 4.8, PTEN activity towards $\mathrm{PIP}_{3}$ displayed a Michaelis-Menten relationship for both the EC and LT enzyme, with maximal activities peaking in the substrate range of $25 \mu \mathrm{M}$. Although the maximal phosphatase activity observed was not significantly different between EC and LT when normalized to total PTEN protein expression, the affinity for $\mathrm{PIP}_{3}$ was nearly 2.7-fold greater during LT (Table 4.1). This suggests that PTEN is more catalytically active 
during LT. This increase in catalytic efficiency was correlated with the higher proportion of non-phosphorylated PTEN enzyme during LT. This linkage between phosphorylation and activity has previously been shown, where the intrinsic activity of PTEN towards $\mathrm{PIP}_{3}$ is regulated by modifications to the carboxy-terminal domain (Odriozola, et al., 2006). It has been previously proposed that phosphorylation on the C-terminal residues promote an electrostatic interaction between three of five PTEN domains, where a closed conformation is formed between the $\mathrm{C} 2$ domain, $\mathrm{C}$-terminal, and the acidic tail, thereby reducing PTEN phosphatase activity (Vazquez et al., 2001). While in its nonphosphorylated form, PTEN exhibits an open conformation, where the phosphatase domain can easily access the substrate at the plasma membrane, assisted by the binding of the N-terminal PBD domain to the acidic surfaces of the cell membrane (Vazquez et al., 2001; Song et al., 2012). The increased catalytic activity of PTEN during LT would further suggest that the reduced level of PDK-1 phosphorylation is likely a result of an increased dephosphorylation of $\mathrm{PIP}_{3}$. Previous chapters have already shown that Akt and components of the mTORC1 network are suppressed during torpor, as a potential mechanism for translational arrest to promote energy conservation (See Chapter 3). The observations here also correlate with previous findings of reduced Akt phosphorylation during torpor (Cai et al., 2004; Chapter 3), and suggest that increased catalytic activity of PTEN during LT serves as an upstream regulator aimed at suppressing downstream components of the insulin signaling pathway. 


\subsubsection{Structural Stability of PTEN}

An increase in catalytic activity of PTEN has previously been associated with a decrease in protein stability, whereby the structural conformation of activated PTEN is more prone to a rapid degradation. This was confirmed in this study, the structural stability of PTEN isolated from LT was significantly lower than during EC. Using partially purified PTEN proteins obtained via Heparin chromatography (FIG. 4.9), the structural stability of PTEN was evaluated with respect to urea denaturation, using the pulse-proteolysis method. This method uses pre-incubation with different concentrations of urea to induce protein unfolding, followed by treatment with thermolysin protease to hydrolyze unfolded protein (depicted in FIG. 4.12) (Park and Marqusee., 2005). As seen in FIG 4.10, the relative amount of native protein remaining decreased with increasing urea concentration and showed that the enzyme from LT was significantly more susceptible to urea denaturation with a $\mathrm{K}_{\mathrm{ND}}$ value of $1.29 \pm 0.06 \mathrm{M}$ urea as compared to EC $(1.62 \pm 0.05 \mathrm{M}$ urea).

The decrease in PTEN stability during LT along with its increased affinity for $\mathrm{PIP}_{3}$ substrate suggests that the PTEN mechanism of regulation is conserved between ground squirrels and other models previously described (Vazquez et al., 2000, 2001). This paradoxical observation of increased catalytic function with decreased stability suggest that the conformational changes caused by phosphorylation also result in diminished inhibitory effects (likely through conformation changes that promote substrate binding) and exposure of potential proteolytic cleavage sites in the enzyme (Vazquez et al., 2000). Using the epestfind bioinformatics tool, potential proteolytic sites 
in the ground squirrel PTEN amino acid sequence were predicted; two major PEST (proline, glutamic acid, serine, and threonine) motifs were identified (FIG. 4.11). The PEST motif is a sequence of polypeptides rich in four specific amino acids that serve as proteolytic signals to promote degradation of proteins by the $26 \mathrm{~S}$ proteasome or $\mathrm{Ca}^{2+}$ activated proteases (Rechsteiner and Rogers, 1996). The second motif predicted in FIG. 4.11 overlaps amino acid residues 375-395, spanning the four key phosphorylation residues (Ser-380, Thr-382, Thr-383, and Ser-385) that have been shown to regulate PTEN activity and stability. As previously described, the phosphorylated form of PTEN possess a closed structural conformation, hiding the PEST motif that is otherwise exposed in the open, non-phosphorylated form of PTEN (Vazquez et al., 2000; Vazquez et al., 2001; Ross and Gericke, 2009). A similar model would also apply to the different modified forms of PTEN observed between EC and LT during squirrel hibernation. Shown in FIG. 4.13, PTEN is likely to be phosphorylated at a basal level in euthermic ground squirrels, where a structurally stable PTEN with reduced activity regulates an active insulin signaling pathway in the presence of $\mathrm{PIP}_{3}$. In torpid ground squirrels, the phosphorylation of PTEN falls below the basal level, and the increased catalytic activity of PTEN promotes the dephosphorylation of $\mathrm{PIP}_{3}$ to disrupt insulin signaling, at a cost of a decreased half-life of the protein.

\subsection{CONCLUSION}

The present chapter details the mechanism that regulates the structure and function of the PTEN phosphatase during torpor in ground squirrels. It was concluded that phosphorylation of residues at the C-terminal of PTEN likely regulates a 
conformational change that increases substrate accessibility to PTEN, although exposing the PEST motif that would increase degradation of the protein. The increased affinity of PTEN for its $\mathrm{PIP}_{3}$ substrate during LT along with decreased phosphorylation of both PTEN and PDK-1 can be viewed as a potential mechanism by which Akt is suppressed during torpor. Overall, the findings here establish PTEN as an upstream regulator that is activated to suppress PDK-1, and subsequently Akt, during torpor and functions as a potential mechanism to reduce insulin signaling during hibernation. 


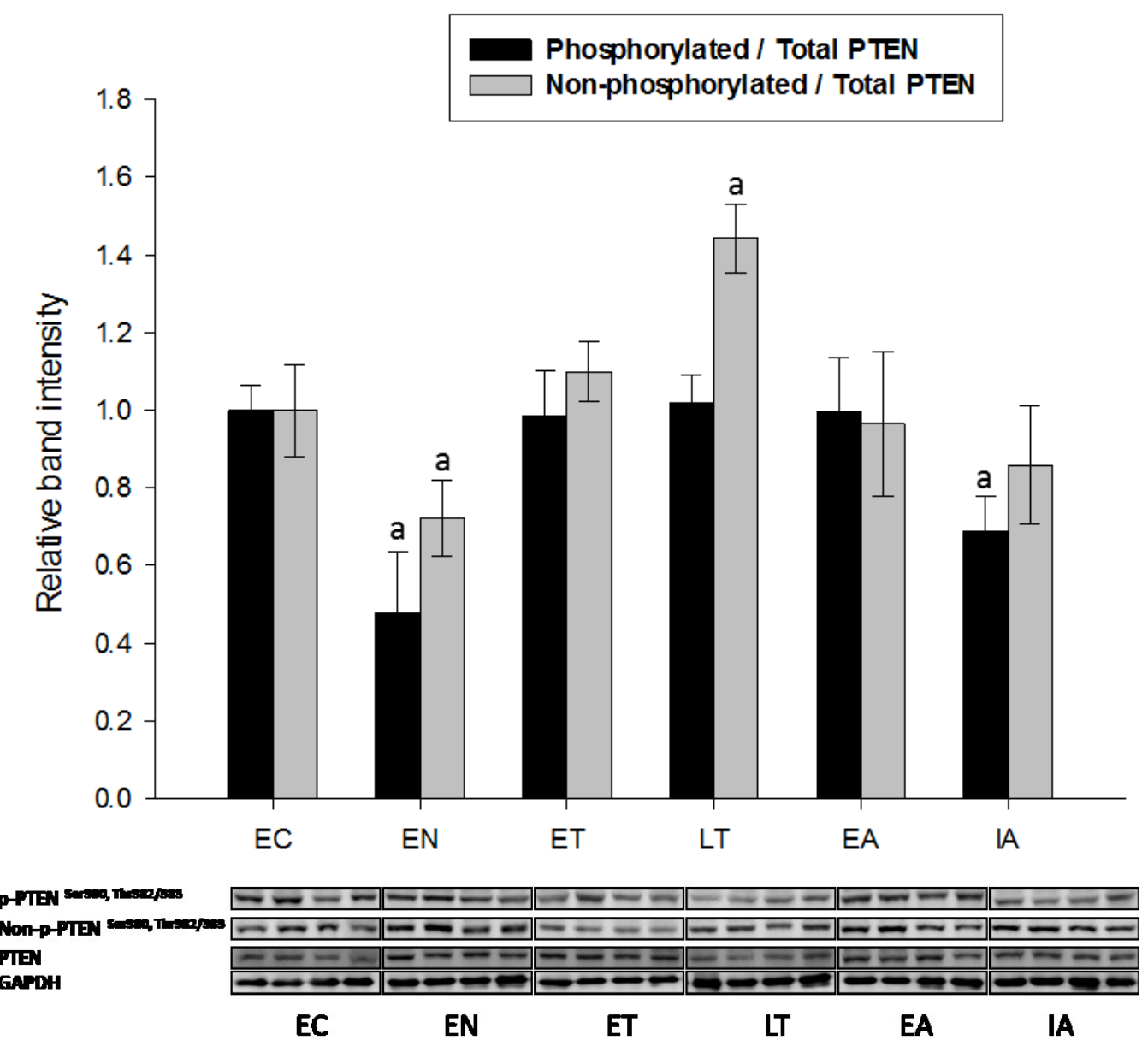

FIG. 4.1. Regulation pattern of PTEN in skeletal muscle of I. tridecemlineatus as determined by Western immunoblotting.

Relative ratio of phosphorylated and non-phosphorylated PTEN to total protein expression of PTEN over the torpor-arousal cycle. Western blot bands show PTEN proteins detected at $\sim 54 \mathrm{kDa}$. Blots of the housekeeping protein GAPDH are shown for protein standardization. Data are mean \pm S.E.M. $(\mathrm{N}=4-5)$ independent trials on tissues from different animals. a-Significantly different from corresponding EC values $(\mathrm{P}<0.05$, ANOVA post hoc Holm-Sidak test). 


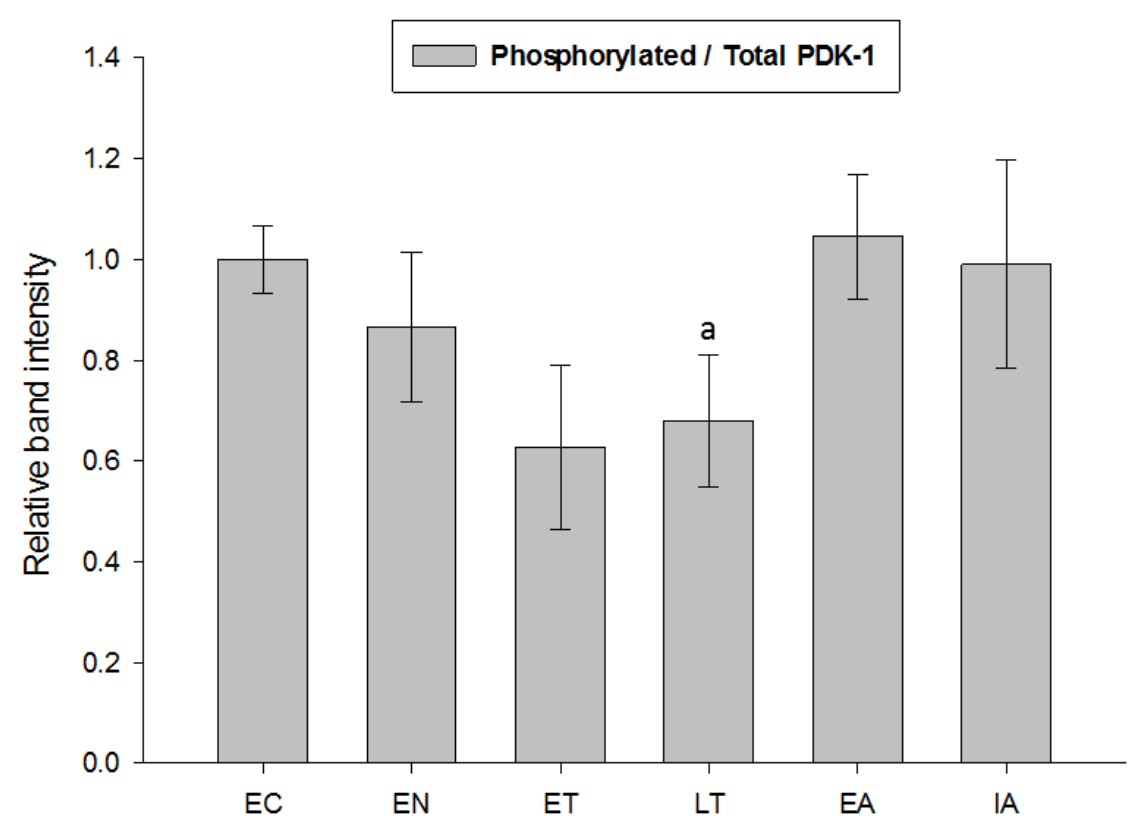

PPDK-1

PDI -1

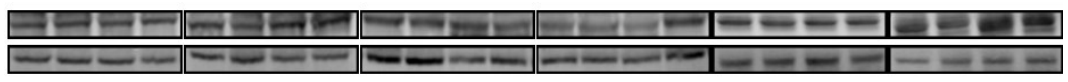

FIG. 4.2. Regulation pattern of PDK-1 in skeletal muscle of I. tridecemlineatus as determined by Western immunoblotting.

Relative ratio of phosphorylated PDK-1 to total PDK-1 protein expression over the torpor-arousal cycle. Western blot bands show PDK-1 proteins detected at $\sim 70 \mathrm{kDa}$. Data are mean \pm S.E.M. $(\mathrm{N}=4-5)$ independent trials on tissues from different animals. a-Significantly different from corresponding EC values $(\mathrm{P}<0.05$, ANOVA post hoc Holm-Sidak test). 


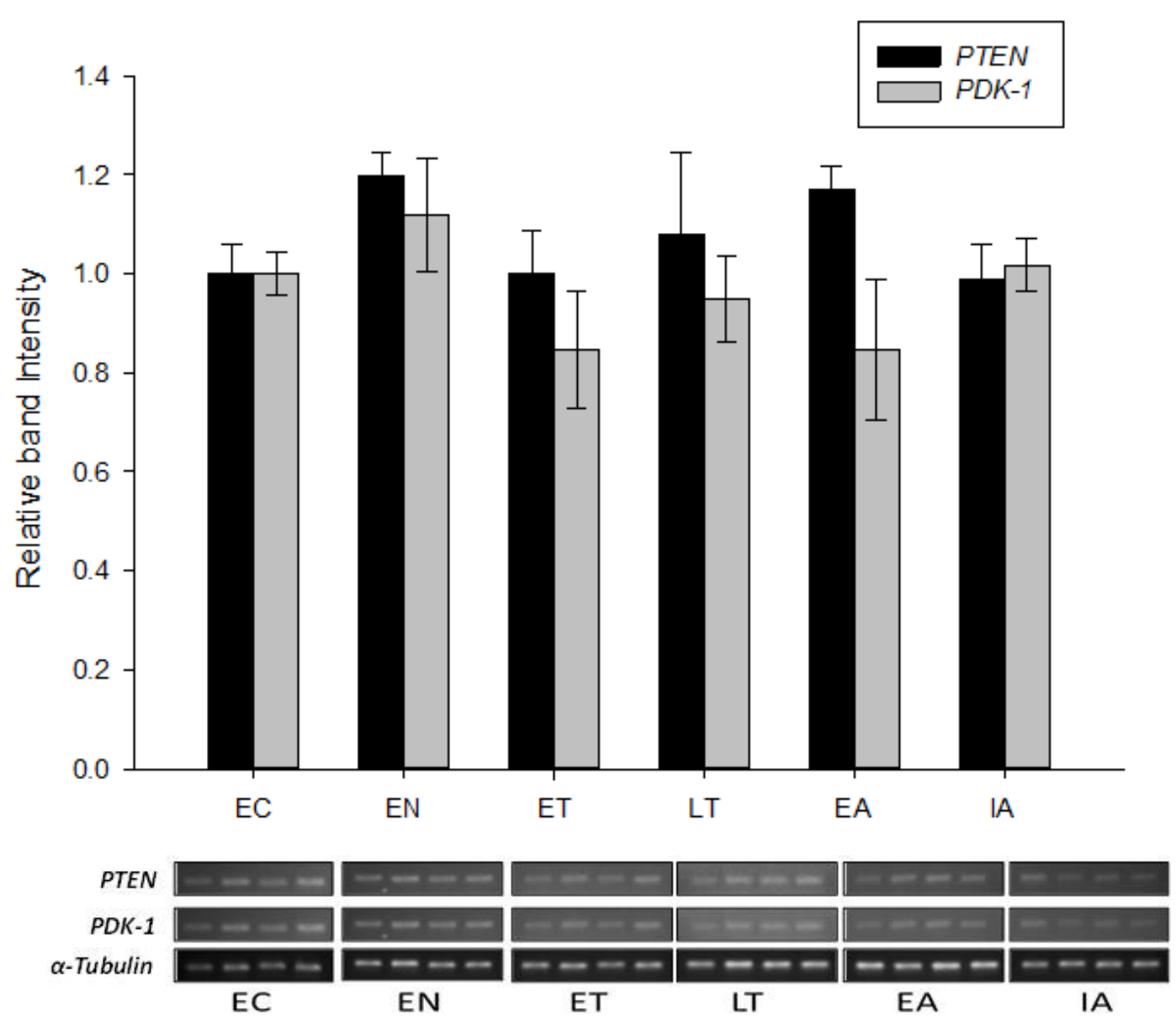

FIG. 4.3. Transcriptional regulation of $P T E N$ and $P D K-1$ in skeletal muscle of $I$. tridecemlineatus as determined by RT-PCR.

Relative expression of PTEN and PDK-1 transcript levels over the torpor-arousal cycle. DNA amplicons are shown along with corresponding bands for the housekeeping gene $\alpha$-tubulin amplified from the same samples. Relative PTEN and PDK-1 band intensities were standardized against corresponding $\alpha$-tubulin band intensities. Data are means \pm SEM, $\mathrm{n}=4-5$ independent trials on tissues from different animals. 
A

\section{FIG. 4.4. Partial cDNA sequence of PTEN}

Partial nucleotide sequence of PTEN amplified from muscle of I. tridecemlineatus and translated amino acid sequence. Sequences closely matched those of human, mouse and rat. 
(A)

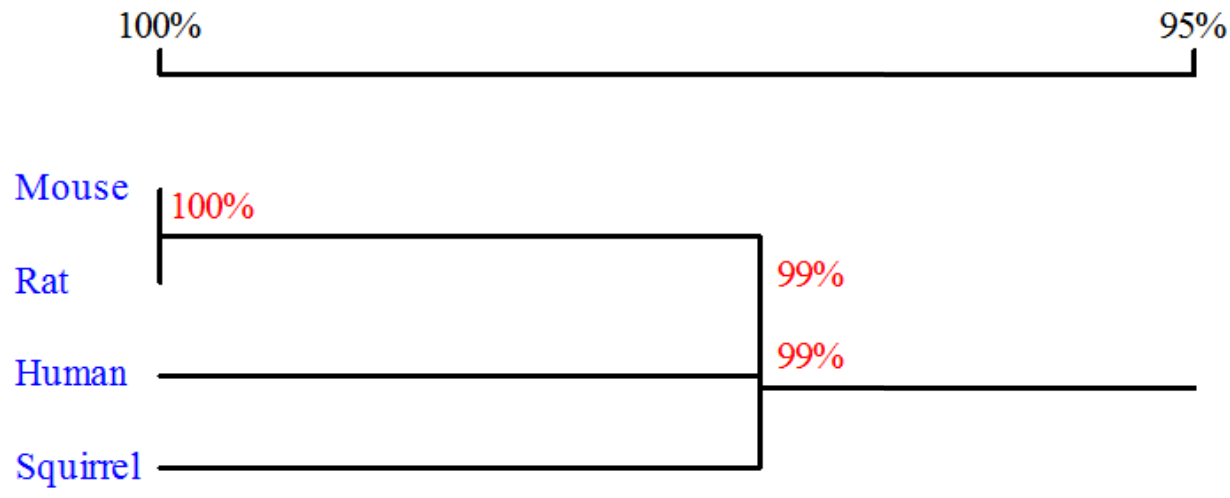

(B)

Mouse Rat Human Squirrel

Mouse

Rat

Human

Squirrel
$100 \%$

$99.7 \% 100 \%$

$99.3 \% 99.0 \% 100 \%$

$99.3 \% 98.9 \% 98.9 \% 100 \%$

\section{FIG 4.5. PTEN homology alignment}

(A) Homology tree produced from alignment of I. tridecemlineatus PTEN protein sequence with human (Homo sapiens), rat (Rattus norvegicus), and mouse (Mus musculus). The percentage values correspond to degree of homology between species. (B) Homology matrix representing degree of conservation of I. tridecemlineatus PTEN sequence compared with PTEN from other mammals. 


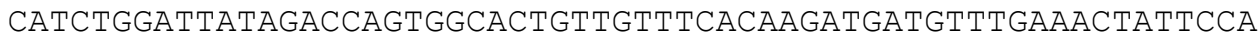

\section{FIG. 4.6. Partial cDNA sequence of $P D K-1$}

Nucleotide sequence of $P D K-1$ amplified from muscle of $I$. tridecemlineatus and translated amino acid sequence. Sequences closely matched those of human, mouse and rat. 
(A)
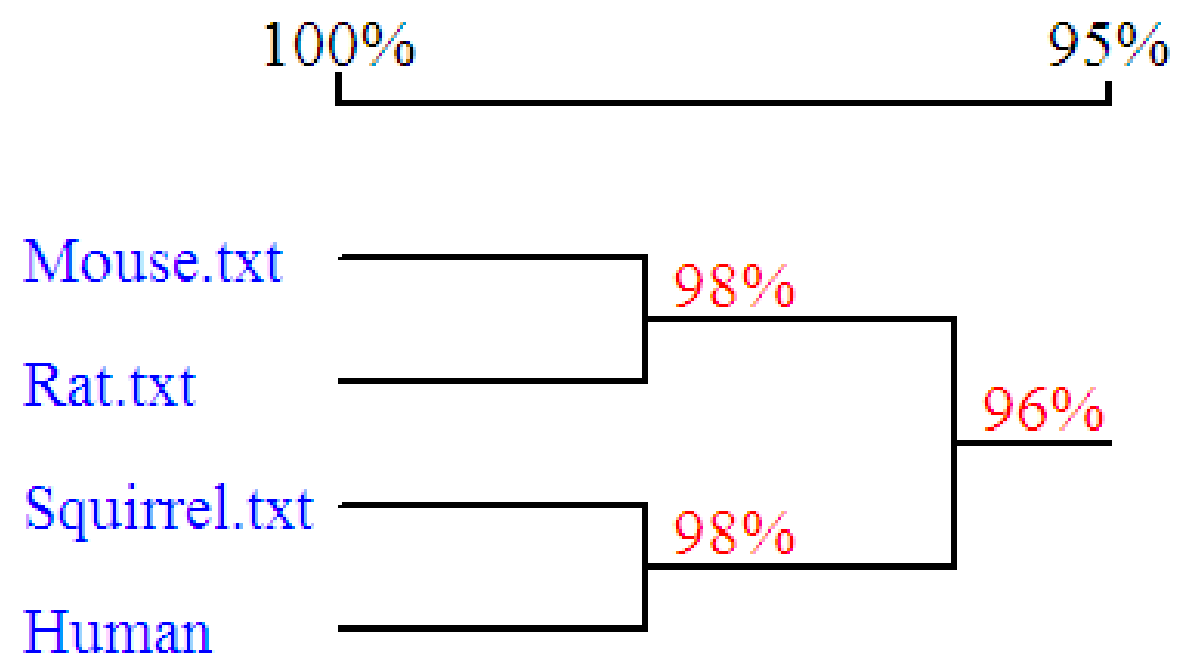

(B)

Mouse Rat Human Squirrel

Mouse

Rat

Human

Squirrel
$100 \%$

$97.9 \% 100 \%$

$75.4 \% 76.1 \% 100 \%$

$96.2 \% 96.8 \% 92.4 \% 100 \%$

\section{FIG 4.7. PDK-1 homology alignment.}

(A) Homology tree produced from alignment of I. tridecemlineatus PDK-1 protein sequence with human (Homo sapiens), rat (Rattus norvegicus), and mouse (Mus musculus). The percentage values correspond to the degree of homology between species. (B) Homology matrix representing degree of conservation of I. tridecemlineatus PDK-1 sequence compared with PDK-1 from other mammals. 


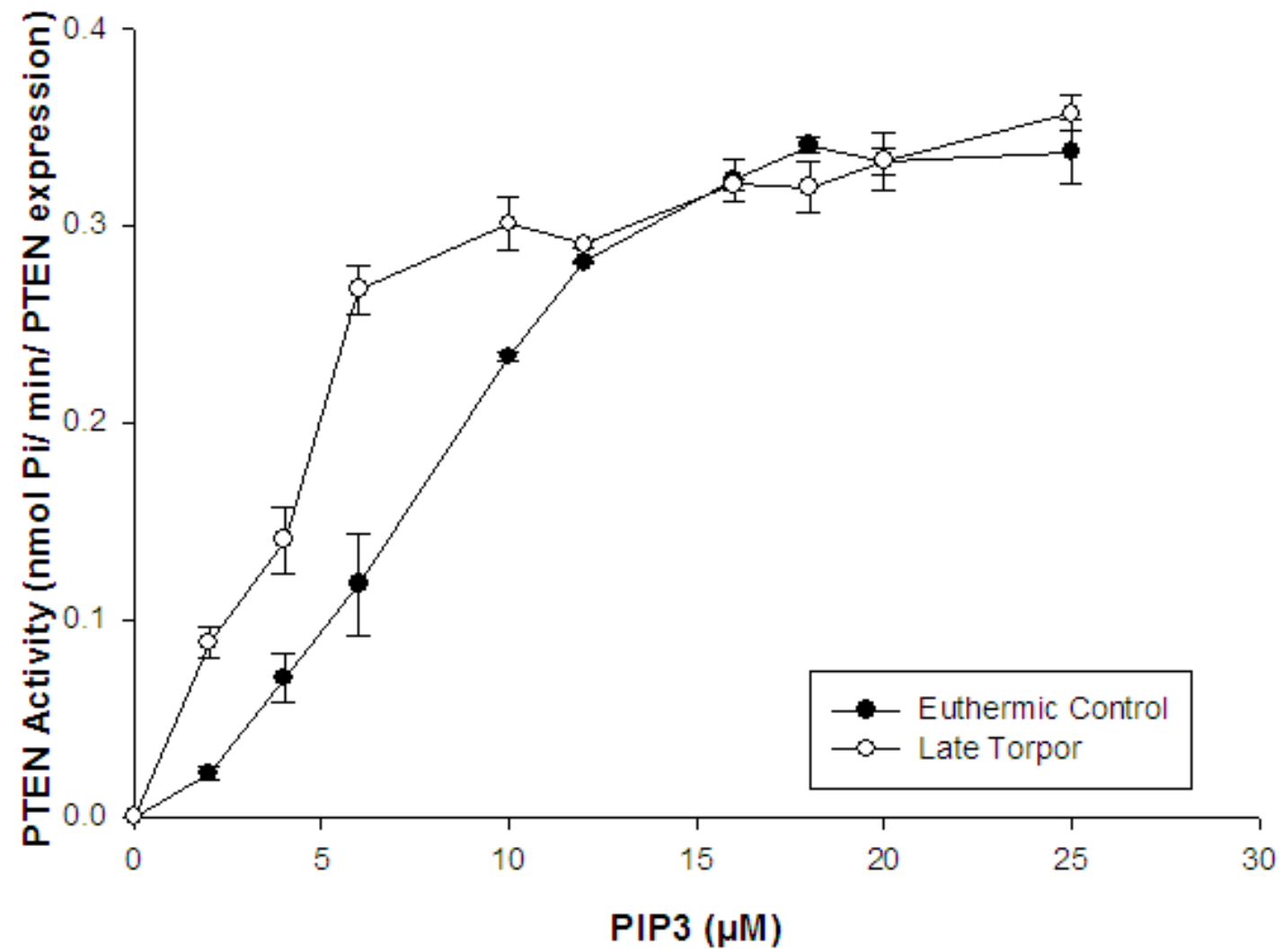

\section{FIG. 4.8. Michaelis-Menten kinetics of PTEN phosphatase activity from skeletal} muscle of I. tridecemlineatus as determined by the malachite green assay.

PTEN activities are shown as a function of $\mathrm{PIP}_{3}$ concentration comparing the skeletal muscle enzyme from euthermic control and late hibernation stages. Activity of PTEN is defined as nmol free phosphate released / min (U), normalized to total PTEN expression as determined by Western blot. Data are means \pm S.E.M. ( $N=4$ independent trials on tissues from different animal). 
Table 4.1. Kinetic parameters of PTEN from skeletal muscle of euthermic and hibernating ground squirrels. Substrate affinity and maximal enzyme velocity for PIP3 is shown, along with $\mathrm{I}_{50}$ urea as determined by pulse-proteolysis assay. Data are mean \pm s.e.m., $\mathrm{n}=4$ independent trials on tissues from different animals. a-Significantly different from the corresponding euthermic value, Student's t-test $(\mathrm{P}<0.05)$.

\section{Euthermic Control}

\section{Late Torpor}

$$
\operatorname{Km~PIP}_{3}(\mu M)
$$

$$
8.96 \pm 1.17
$$

$3.96^{\mathrm{a}} \pm 0.47$

$\boldsymbol{V}_{\boldsymbol{m a x}}($ nmol Pi/min/PTEN)

$0.49 \pm 0.04$

$0.42 \pm 0.01$

$\boldsymbol{K}_{N D} \operatorname{Urea}(M)$

$1.62 \pm 0.05$

$1.29^{\mathrm{a}} \pm 0.06$

$$
1.29^{\mathrm{a}} \pm 0.06
$$




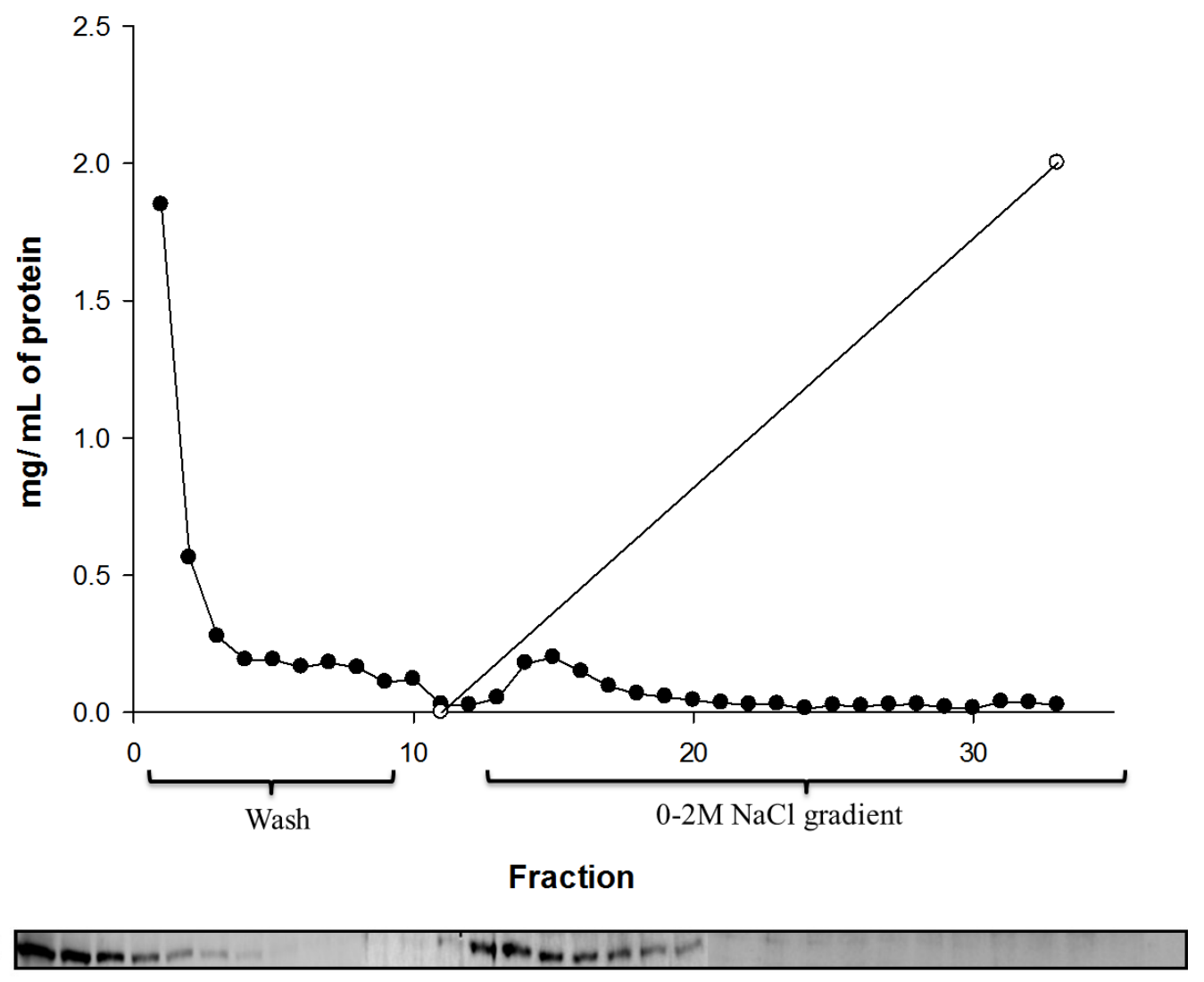

FIG. 4.9. Elution profile of euthermic control proteins from Heparin affinity chromatography.

Graph shows the elution profile of total protein over a linear 0-2 $\mathrm{M}$ gradient of $\mathrm{NaCl}$; protein concentration $(\mathrm{mg} / \mathrm{ml})$ was determined using the Bradford assay. Elution of PTEN protein was determined by Western immunoblot analysis of the fractions collected. 

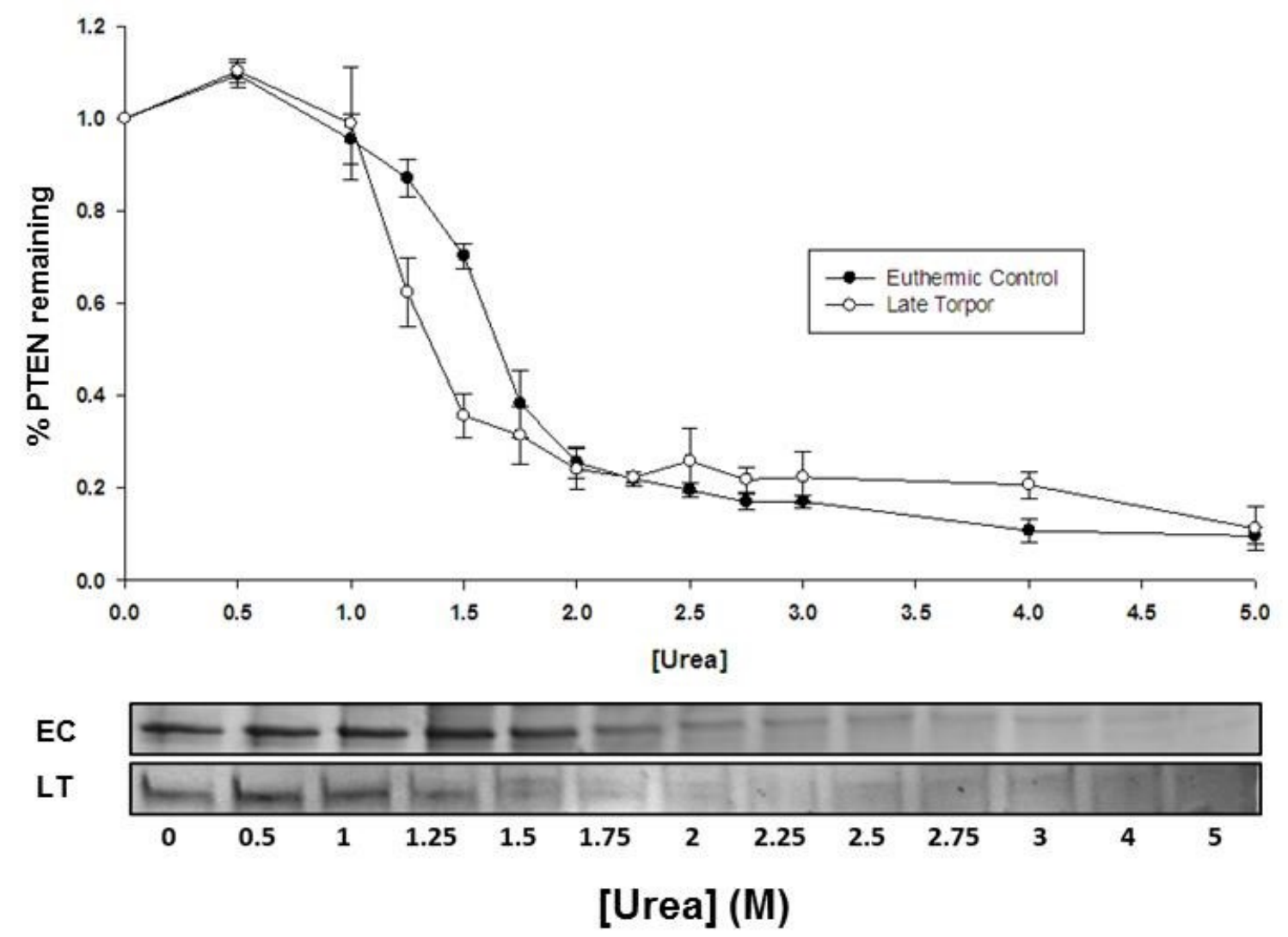

FIG. 4.10. Analysis of I. tridecemlineatus PTEN structural stability as determined by pulse-proteolysis.

Unfolding of purified PTEN as assessed from the relative intensities of native protein remaining (band showed at $54 \mathrm{kDa}$ ) after incubation with different concentrations of urea and a 10 minute treatment with thermolysin to hydrolyze denatured protein.

Subsequently, the amount of native protein remaining was analyzed by electrophoresis of samples on a $10 \%$ SDS-PAGE gel, and visualized by silver staining. Data are means \pm S.E.M. ( $\mathrm{N}=4$ independent trials on tissues from different animal). 
1 MTAIIKEIVSRNKRRYQEDGFDLDLTYIYPNI IAMGFPARLEGVYRNNIDDVVRFLDSKH 60

61 KNHYKIYNLCAERHYDTAKFNCRVAQYPFEDHNPPQLELIKPFCEDLDQWLSEDDNHVAA 120

121 IHCKAGKGRTGVMICAYLLHRGKFLKAQEALDFYGEVRTRDKKGVTIPSQRRYVYYYSYL 180

181 LKNHLDYRPVALLFHKMMFETIPMFSGGTCNPQFVVCQLKVKIYSSNSGPTRREDKFMYF 240

241 EFPQPLPVCGDIKVEFFHKQNKMLKKKMFHFWVNTFFIPGPEETSERVENGSLCDQEIDS 300

301 ICSIERADNDKEYLVLTLTKNDLDKANKDKANRYFSPNFKVKLYFTKTVEEPSNPEASSS 360

$++++++++++++t$

361 TSVTPDVSDNEPDHYRYSDTTDSDPENEPFDEDQHTQITKV 401

$+++++++++++\quad+++++++++++++++$

Symbols PEST motifs

++++++ potential

FIG. 4.11. Prediction of PEST motif from the amino acid sequence of $I$.

tridecemlineatus PTEN retrieved from ensemble database (ENSSTOT00000028610) using the epestfind bioinformatics program.

Potential sites of proteolytic cleavage as predicted via PEST motif were predicted using the bioinformatics tool epestfind. Two potential PEST motifs were found in the amino acid sequence between position 347-373 and 377-394, highlighted in bold with + symbol. Phosphorylation residues of Ser-378, Thr-380, Thr-381 are shaded in grey. 

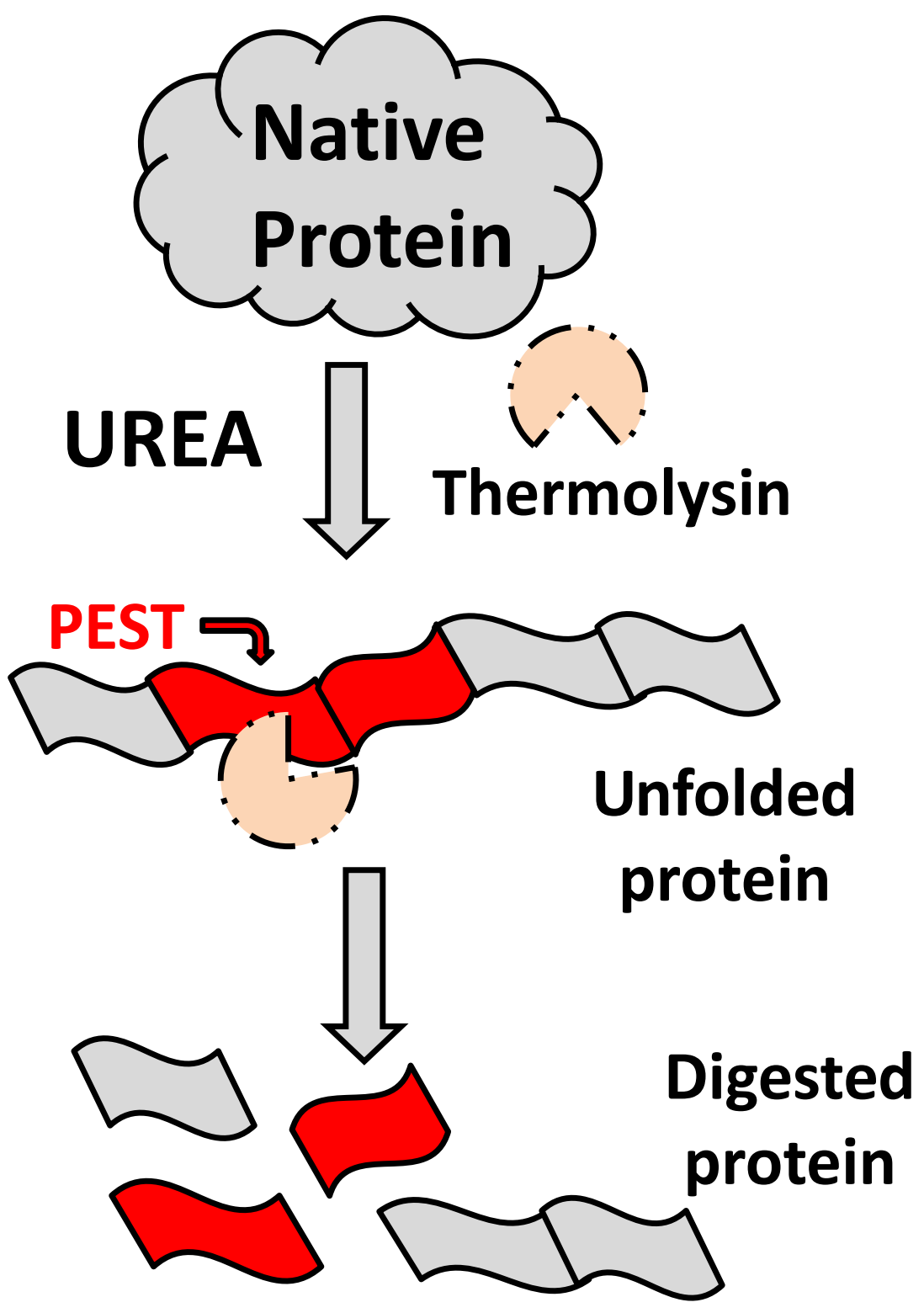

FIG. 4.12. Mechanism of pulse-proteolysis method

Native proteins are incubated with various concentrations of urea to induce unfolding of protein structures, leading to the exposures of potential PEST motifs, facilitating rapid protein degradation in the presence of proteases. 


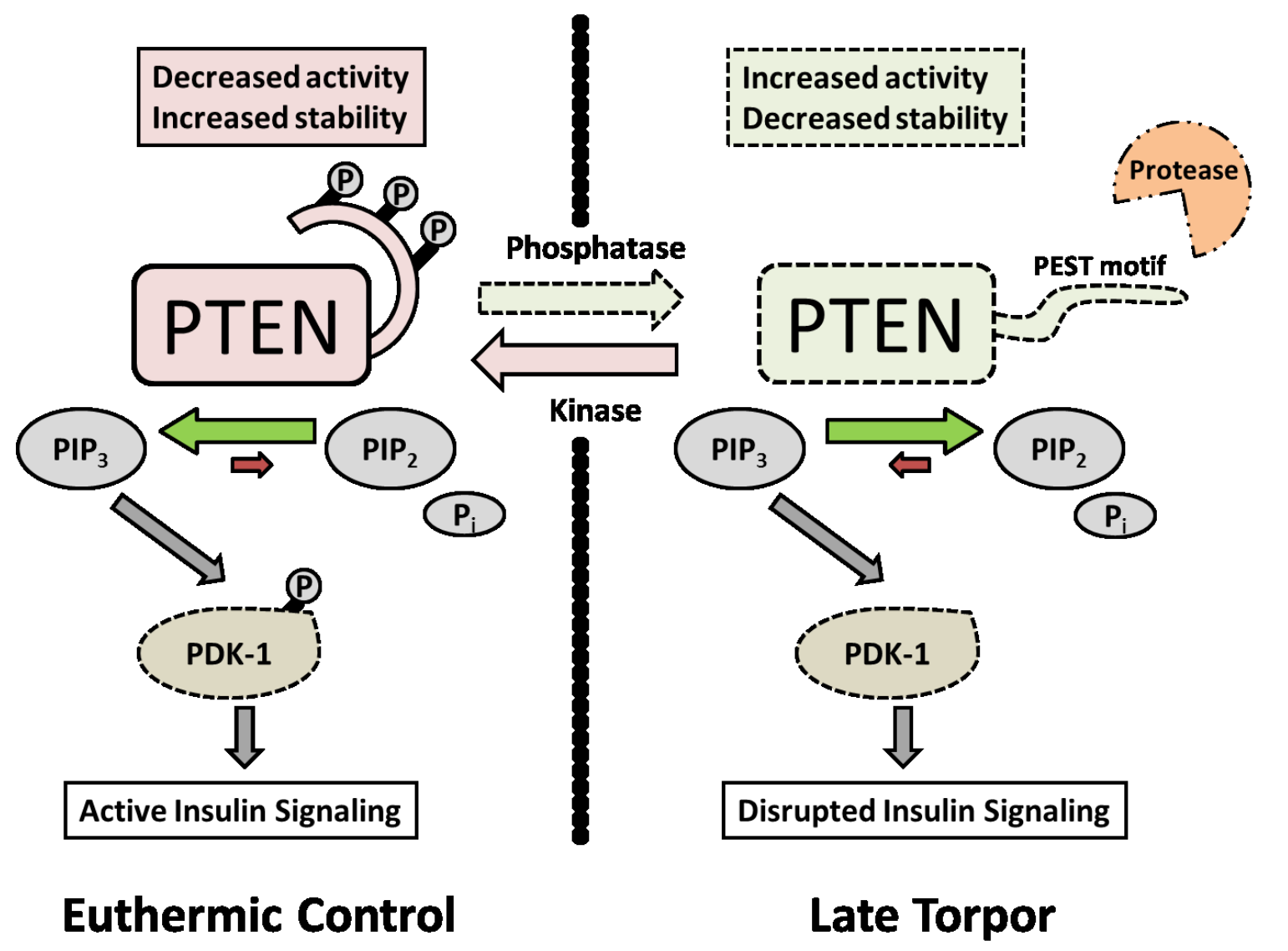

FIG. 4.13. Proposed mechanisms of PTEN regulation between euthermia and torpor.

The C-terminal "tail" domain of PTEN is phosphorylated at multiple residues, and is critical in maintaining protein stability as well as inhibiting PTEN function. When phosphorylated at Ser380, Thr382/383, PTEN is functionally inhibited and so in the presence of PIP3, PDK-1 is activated via phosphorylation at Ser-241 to promote the insulin signaling pathway. Upon dephosphorylation, PTEN is functionally active, catalyzing the conversion of $\mathrm{PIP}_{3}$ to $\mathrm{PIP}_{2}$, thereby inhibiting PDK-1 activation, and attenuating the insulin signaling pathway. However, PTEN dephosphorylation has been shown to lead to reduced protein stability, through the potential exposure of PEST motifs, promoting proteolytic cleavage of PTEN. 


\section{CHAPTER 5}

SMAD MEDIATED TRANSCRIPTIONAL

RESPONSE DURING HIBERNATION 


\subsection{INTRODUCTION}

Biochemical pathways can often be effectively regulated by the control of selected key modulators. This is particularly important in regulating signalling transduction pathways, which are typically initiated via changing the levels of an upstream molecule with effects that are then amplified and directed to elicit a biological response. Although hibernation clearly requires global controls for overall suppression of metabolic rate, differential regulation of various metabolic pathways is also required to reprioritize energy expenditures on different metabolic functions. A pathway of particular interest is the transforming growth factor- $\beta$ (TGF- $\beta$ ) pathway. The TGF- $\beta$ signalling pathway activates a family of transcription factors (Tf) named Smad (small mothers against decapentaplegic). Once activated, Smads translocate into the nucleus and interact with several effector proteins, leading to the activation or repression of different target genes (Massaguè et al., 2005). The transactivating property of a Smad affects the expression of its downstream gene target by either promoting or suppressing the transcription of the gene. Typically described as an anti-proliferatory signaling pathway, downstream targets of Smads that are of particular interest include several that are key regulators of other cellular processes including c-myc, growth arrest and DNA-damageinducible-beta (GADD45 $\beta$ ) protein, death associated protein kinase 1 (DAP-Kinase), cell cycle inhibitor proteins $\mathrm{p} 15 \mathrm{INK} 4 \mathrm{~b}$ and $\mathrm{p} 21 \mathrm{CIP} 1$, and a number of microRNAs involved in metabolic regulation (Massaugè et al., 2005; Kong et al., 2008).

TGF- $\beta$ membrane bound receptors can interact with a number of different cytokines, each group of cytokines leading to the activation of a different signal mediator 
(Massaugè, 2000). The TGF- $\beta$ cytokine is one of the many stimuli that can initiate this receptor mediated signalling pathway; other family members such as bone morphogenetic proteins (BMP), Nodals, activins, myostatins, and anti-Muellerian hormone (AMH) can also initiate downstream signal mediators by binding with TGF- $\beta$ receptors (Massaguè, et al., 2005). There are two types of TGF- $\beta$ membrane receptors, type I and type II (FIG 5.1). The mechanism of ligand-receptor interaction has been well-studied, and it has been shown that both receptors are required for proper ligand binding and subsequent signalling. Upon binding of stimuli, the type I and II receptors dimerize and function as a serine/threonine kinase that phosphorylates intracellular receptor Smads (R-Smads). The R-Smads (Smad 2 and Smad 3) are unique in containing a Ser-Ser-Val/Met-Ser (SSXS) sequence motif in their C-termini, and phosphorylation of the serine residues in this motif is critical to activation (Wu et al., 2001). Upon phosphorylation of R-SMADs at the SSXS motif, they form a heteromeric complex with common Smad 4 (Co-Smad), and translocate into the nucleus. The Mad Homology domain (MH1), found in both RSMADs and Co-SMAD is characterized by its $\beta$-hairpin structure, which possesses sequence-specific DNA binding affinity (Shi \& Massaugè, 2003). This domain is thought to be critical to the transcriptional activity of the nuclear SMAD complex.

In the nucleus, the Smad 2/3-Smad 4 complex interacts with DNA through the Smad Binding Element (SBE), 5'-GTCT-3', in the promoter region of selected genes. For example, two targets containing a SBE are the proteins p15INK4b and p21CIP1, two key cell cycle inhibitors (cyclin-dependent kinase inhibitor; $\mathrm{CKI}$ ) that regulate the early phases of cell cycle progression $\left(\mathrm{G}_{1}\right.$ and $\mathrm{S}$ phase) (Nakayama and Nakayama, 1998). p15INK4b interacts with Cdk 4, stalling the cell cycle in the $\mathrm{G}_{1}$ phase whereas p21CIP1 
forms an inhibitory complex with cyclin E: Cdk2, stalling the cell cycle at the $\mathrm{G}_{1} \rightarrow \mathrm{S}$ transition phase.

The Smad family of transcription factors is also linked to regulation of microRNAs. MicroRNAs are short-length double-stranded RNA molecules of $\sim 21-25$ nucleotides in length. They were first discovered in Caenorhabditis elegans through genetic screening, and are now known to be highly conserved across phylogeny (Hudder \& Novak, 2008). MicroRNAs have roles in the post-transcriptional regulation of mRNA transcripts and are involved in many cellular processes including at virtually all stages of development. Through bioinformatic screening, it has been predicted that microRNAs can regulate up to $30 \%$ of the human genes. The microRNA regulatory network is a complex system, since a single microRNA can interact with multiple mRNA transcript types, and a single mRNA type can be regulated by multiple microRNAs. Formation of complexes between mRNA and microRNA has two potential effects on the mRNA transcript: targeting the mRNA for destruction by endonucleases or translational suppression of the mRNA by binding it into a silencing complex. The deciding factor in the fate of the mRNA is the degree of complementarity between the mRNA and microRNA, which determines the strength of interaction. Kong et al. (2008) have previously identified possible microRNAs that are under the control of TGB- $\beta$ signalling through the SMAD Tfs. Several microRNAs have been shown to either increase or decrease in expression in the presence versus absence of SMAD 4 (Kong et al., 2008). Those that increased included miR-155, miR-21, and miR-29b, which negatively regulate expression of many metabolic targets such as Cdc25A in cell cycle, PI3-K and PTEN phosphatase in insulin signaling (Wang et al., 2009; Wang et al., 2011; Park et al., 2009). 
The TGF- $\beta$ signalling pathway is known to be crucial in the development of $C$. elegans, specifically during the alternative L3 larval stage, also known as the dauer stage (Inoue \& Thomas, 2000). The dauer stage is considered to be a survival mode that is induced when the environment is unfavourable for continuing development. Environmental factors that influence the induction of dauer include food availability, population density, and habitat temperature (Riddel et al., 1981). Parallels can be drawn between the C. elegans dauer stage and mammalian hibernation, both of which are forms of hypometabolism that are induced by the organism when the habitat is unfavourable for survival. The present chapter examined of the regulation pattern of the Smad family of transcription factors over the torpor-arousal cycle in ground squirrel hibernation. Due to the growth suppressive roles that Smad play in hypometabolism, I hypothesize that activation of Smad transcription factors will be evident during torpor, potentially functioning as a mechanism to promote suppression of cell cycle progression and insulin signaling through downstream activation of CKIs and microRNAs.

\subsection{RESULTS}

\subsubsection{Protein expression of Smad 2, 3, and 4.}

Information about the regulation of target proteins at the translational level can be gained by measuring protein expression levels using Western blotting, a technique that utilizes antibodies that recognize specific peptide sequences of target antigens. To determine if Smad family proteins are regulated during the torpor-arousal cycle, immunoblot analysis was carried out for Smad 2, Smad 3 and Smad 4, examining protein levels in ground squirrel liver and skeletal muscle over six stages of the torpor-arousal 
cycle (FIG. 5.2- 5.4). Smad 4 functions as a transporter protein that forms a complex with Smad 2 or Smad 3 to facilitate their translocation into the nucleus. The polyclonal Smad 4 antibody recognizes a protein band at $\sim 70 \mathrm{kDa}$, the expected molecular mass of the protein in other mammals. In FIG. 5.2, tissue specific responses were observed for Smad 4 protein levels between liver and skeletal muscle. In skeletal muscle, total protein expression of Smad 4 was unchanged across the torpor-arousal cycle. However, in liver, a $24 \%$ reduction in Smad 4 levels occurred as squirrels entered torpor (EN), followed by a subsequent increase by 1.19-fold and 1.38-fold in ET and LT, respectively, compared to EC. However, during IA, expression of Smad 4 was again reduced by $56 \%$ compared to $\mathrm{EC}(\mathrm{P}<0.05)$

As seen in FIG. 5.3, tissue specific regulation patterns of Smad 2 protein were also apparent. The polyclonal Smad 2 antibody cross-reacted with a protein band at $\sim 60$ $\mathrm{kDa}$, the expected size of mammalian Smad 2. In skeletal muscle, expression of Smad 2 protein did not change significantly across the torpor-arousal cycle. However, in liver, a significant reduction in Smad 2 protein expression was observed during LT and IA by $71 \%$ and $87 \%$ respectively compared to EC (both $\mathrm{P}<0.05$ ).

The polyclonal Smad 3 antibody cross reacted with a protein band at $\sim 52 \mathrm{k}$ Da. As shown in FIG. 5.4, the expression of Smad 3 protein in skeletal muscle showed a similar pattern to that for Smad 2, with constant expression over the torpo- arousal cycle. However, in the liver, a strong significant reduction in Smad 3 total protein level occurred in the EN phase of the torpor-arousal cycle, levels decreasing by $71 \%$ as 
compared to $\mathrm{EC}(\mathrm{P}<0.05)$. However, Smad 3 protein expression was restored back to $\mathrm{EC}$ values in ET, and remained stable over the remainder of the torpor-arousal cycle.

\subsubsection{Protein expression of Smurf 2 ubiquitin ligase}

Smad family proteins are post-translationally regulated by an ubiquitin ligase called Smurf 2 that tags Smads with ubiquitin to mark them for degradation by the proteasome. Immunoblotting was used to assess changes in Smurf 2 protein levels over the torpor-arousal cycle. As seen in FIG. 5.5, total protein levels of Smurf 2 were reduced by $33 \%$ in skeletal muscle during LT followed by a 1.27 -fold increase in EA, and another reduction by $38 \%$ in IA, all as compared to $\mathrm{EC}(\mathrm{P}<0.05)$. In the liver, minimal changes were observed during the torpor-arousal cycle, with a significant suppression of Smurf 2 by $31 \%$ only during IA, compared to EC $(\mathrm{P}<0.05)$.

\subsubsection{Phosphorylation of Smad 2 and Smad 3.}

The receptor Smad proteins (Smad 2 and 3) are post-translationally activated via phosphorylation on selected amino acid residues, and the relative phosphorylation state can be assessed using antibodies that recognize specific peptide sequences that contain the crucial phosphorylated amino acid residues. Changes in the phosphorylation of Smad 2 and Smad 3 proteins over the torpor-arousal cycle were assess in both liver and skeletal muscle of ground squirrels via immunoblotting (FIG. 5.6 - FIG. 5.7). As seen in FIG. 5.6, the relative phosphorylation state of Smad 2 at Ser-465 / Ser-467 did not change significantly over torpor and arousal in either tissue. However, phosphorylation Smad 3 showed some changes during hibernation. In liver, phosphorylation on Ser-423 / Ser-425 of Smad 3 was significantly elevated during ET and LT; levels were 1.41-fold and 1.58- 
fold higher, respectively, as compared to $\mathrm{EC}(\mathrm{P}<0.05)$ (FIG. 5.7). In the muscle, a significant 1.55-fold increase in phosphorylation of Smad 3 was observed during EN $(\mathrm{P}<0.05)$

\subsubsection{Nuclear expression of Smad 4 protein and relative phosphorylation of Smad}

\section{2 and Smad 3}

Upon phosphorylation, receptor Smad 2 and Smad 3 form complexes with the common Smad 4 and translocate into the nucleus where the DNA binding activity of Smad 2 and Smad 3 regulates the expression of target genes. To determine whether the changes in phosphorylation of Smad 2 and Smad 3 reported above correlated with changes in the nuclear translocation of these Tfs, immunoblotting was performed to measure the relative levels of each protein in nuclear extracts from liver and muscle sampled from the EC and LT stages (FIG. 5.8). The nuclear content of Smad 4 protein did not change in skeletal muscle between the two states but Smad 4 content in nuclear extracts of liver increased strongly by 3.35 -fold in LT, as compared to EC. The relative amount of phosphorylated Smad 2 (Ser-465 / Ser-467) in nuclear fractions did not change between EC and LT states in either tissue but phosphorylation of Smad 3 (Ser-423 / Ser425 ) increased strongly. The content of phosphorylated Smad 3 increased by 2.85 -fold in nuclear extracts of skeletal muscle during LT and by 2.27 -fold in liver, as compared to EC values $(\mathrm{P}<0.05)$. Although the increase of phosphorylated Smad 3 in liver correlated with a similar increase observed in FIG. 5.7, the increase observed in the skeletal muscle was surprising.

\subsubsection{Transcriptional regulation and cloning of Smad 2}


To determine if the Smad family of proteins was regulated at the transcriptional level during hibernation, RT-PCR analysis was used to measure the levels of Smad mRNA in liver and skeletal muscle of ground squirrels. Changes in the relative transcript levels of Smad 2 over the torpor-arousal cycle are shown in FIG. 5.9; Smad 2 transcripts were standardized against the expression of $\alpha$-tubulin amplified from the same samples. In liver, transcript levels of Smad 2 increased significantly during EA and IA by 1.45 and 1.41-fold, respectively, compared to EC $(\mathrm{P}<0.05)$. By contrast, in skeletal muscle, Smad 2 mRNA levels were down-regulated during LT and EA, falling by 34\% and 23\% as compared to $\mathrm{EC}(\mathrm{P}<0.05)$.

The PCR analysis of Smad 2 yielded an amplicon of $660 \mathrm{bp}$ in length. The amplicon was sequenced and closely matched a portion of an open reading frame (ORF) of the Smad 2 gene from other mammals (FIG. 5.10). The ORF encoded 217 amino acids, a partial sequence of Smad 2 representing $46 \%$ of the full amino acids sequence in mammals. When the partial ground squirrel sequence was compared to the same Smad 2 segment from other mammals, a very high homology was found between the squirrel sequence and the mouse (99.4\%), rat (99.1\%), and human (100\%) sequences (FIG. 5.11).

\subsubsection{Transcriptional regulation and cloning of Smad 3}

Relative levels of Smad 3 mRNA transcripts were assessed by RT-PCR (FIG.

5.12). In the skeletal muscle, Smad 3 mRNA levels rose significantly by 1.99 -fold during EN as compared to EC $(\mathrm{P}<0.05)$, but were stable over the rest of the torpor-arousal cycle. In the liver, Smad 3 mRNA transcripts rose significantly by 1.65 -fold during EA compared to $\mathrm{EC}(\mathrm{P}<0.05)$, while also remaining stable during the other stages. 
The PCR analysis of Smad 3 yielded an amplicon of 619 in length (FIG. 5.13) that closely matched the sequence of Smad 3 open reading frames from other mammals. The translated sequence coded for 206 amino acids. When compared to the same segment of the protein from human, rat and mouse, the homology tree and matrix showed near perfect conservation between the squirrel sequence and mouse (99.8\%), rat (100\%), and human (100\%) (FIG. 5.14).

\subsubsection{Transcriptional regulation and cloning of Smad 4}

Relative levels of Smad 4 mRNA transcripts were determined across the torporarousal cycle but did not change in either liver or skeletal muscle (FIG. 5.15).

The PCR analysis of Smad 4 yielded an amplicon of $994 \mathrm{bp}$ in length. The nucleotide sequence closely matched the open reading frames of Smad 4 from other mammals. The translated sequence produced a peptide of 328 amino acid (FIG. 5.16). As compared to other mammals, the homology tree and matrix showed a high degree of conservation of the squirrel sequence with mouse (99.0\%), rat (99.4\%), and human (99.0\%) Smad 4 (FIG. 5.17).

\subsubsection{EMSA analysis of Smad binding probes and DNA binding activity}

To determine if changes in the nuclear translocation of Smad 3 resulted in increased DNA binding, electrophoretic mobility shift assays were performed. FIG. 5.18 shows a positive interaction (single band shown by arrow) between Smad 3 protein in the nuclear extract and the double stranded DNA probes containing the Smad binding elements (SBE) (lane 3). This compares with the absence of this band in control samples 
lacking either the SBE probe (lane 1) or the nuclear extract (lane 2). To verify that the shifting of the single band observed in lane 3 was a product of Smad 3 protein binding, 'supershift' analysis was performed using phosphorylated Smad 3 polyclonal antibody. As seen in lane 4, incubation with p-Smad 3 antibody resulted in the disappearance of a single band observed in lane 3 . Although supershift analysis should result in a band at a higher location of the gel due to retarded movement through the gel because of the bound antibody, this was not seen. It is possible that the complex did not travel sufficiently into the gel to become resolvable. Importantly, however, the positive interactions of Smad 3 in nuclear samples with SBE probes demonstrated that the same probes could be used in DNA binding assays.

FIG. 5.19 shows an analysis of the DNA binding activity of Smad 3 using the enzyme-linked immunosorbent assay (ELISA) assay and comparing EC and LT conditions. In liver, Smad3 binding was significantly higher during LT, an increase of 1.54-fold compared to euthermic controls $(\mathrm{P}<0.05)$. In skeletal muscle however, no significant change in Smad 3 DNA binding activity was observed between the two states.

\subsubsection{Co-immunoprecipitation of Smad 3 complex}

Although the ELISA data indicated enhanced DNA binding by Smad 3 only in the liver, a co-immunoprecipitation using Smad 3 antibody was performed to determine qualitatively whether a Smad 3:Smad 4 complex formed in either liver or skeletal muscle. As seen in FIG. 5.20, Smad 4 was readily detected in liver samples (lane 2, 3) during both EC and LT, as were both total and phosphorylated Smad 3. However, in skeletal muscle, although the presence of phosphorylated Smad 3 was readily detected, the 
amount of Smad 4 that co-immunoprecipitated was much less than in liver, suggesting decrease presence of a Smad 3: Smad 4 complex.

\subsubsection{Transcriptional regulation of cell cycle inhibitors p15INK4b and p21CIP1}

To determine if regulations of Smad signaling results in the subsequent control of downstream targets, RT-PCR was performed to measure the mRNA expression of $p 15 I N K 4 b$ and $p 21 C I P 1$. Shown in FIG. 5.21, mRNA expression levels of $p 15 N K 4 b$ in liver were elevated by 1.74-fold and 2.08-fold during ET and LT, respectively, as compared to EC $(\mathrm{P}<0.05)$. The expression of $p 21 C I P 1$ in liver was also upregulated by 1.69-fold, 1.51-fold, and 1.52-fold during ET, LT, and IA, respectively, compared to EC $(\mathrm{P}<0.05)$. In skeletal muscle, the mRNA expression level of $p 15 I N K 4 b$ was stable over the torpor-arousal cycle (FIG. 5.22). However, the expression of $p 21 C I P 1$ was upregulated by 2.09-fold and 1.92-fold during LT and EA, respectively, as compared to $\mathrm{EC}(\mathrm{P}<0.05)$.

\subsubsection{1 cDNA cloning of partial squirrel CKI transcripts}

PCR analysis of $p 15 I N K 4 b$ yielded an amplicon of 186 nucleotides in length that closely matched the open reading frames of other mammals (FIG. 5.23). The nucleotide sequence encoded 62 amino acids that showed good homology with non-hibernating

mammals (FIG. 5.24); the percent identity with the ground squirrel sequence was mouse $(88.5 \%)$, rat $(86.3 \%)$, and human $(87.0 \%)$.

The PCR analysis of $p 21 C I P 1$ yielded an amplicon of 228 nucleotides that encoded 76 amino acids (FIG. 5.25). When compared to other mammals, the homology 
was high; conservation between the squirrel sequence and other mammals was mouse $(82.2 \%)$, rat (78.1\%), and human (80.9\%) (FIG. 5.26).

\subsubsection{Expression of Smad regulated microRNA specie}

Stem-loop PCR was used to determine if changes in the DNA binding activity of Smad 3 over the torpor-arousal cycle resulted in changes in the expression of microRNA targets that are under Smad control. Three microRNAs involved in key signaling pathways were examined: miR-21, miR-29b, and miR-155. Shown in FIG. 5.27 are the relative levels of these three microRNAs in ground squirrel liver; the expression of $\alpha$ tubulin that was used for sample standardization is also shown. Relative levels of miR-21 in liver were significantly elevated by 1.53-fold, 1.57-fold, and 2.11-fold in EN, ET and EA, respectively, as compared to EC $(\mathrm{P}<0.05)$. Relative expression of liver miR-29b was elevated by 1.39 -fold and 1.27-fold in ET and LT, respectively $(\mathrm{P}<0.05)$ whereas relative expression of miR-155 was elevated by 1.56 -fold only during $\mathrm{EN}(\mathrm{P}<0.05)$.

A comparable analysis of Smad-regulated microRNA target in skeletal muscle is shown in FIG. 5.28. Relative expression of miR-21 was elevated only during IA by 1.29fold, compared to EC $(\mathrm{P}<0.05)$, whereas the expression of miR-29b was elevated by 1.28-fold in ET $(\mathrm{P}<0.05)$. Transcript levels of miR-155 were stable over the torporarousal cycle.

\subsection{DISCUSSION}

The Smad family of transcription factors are regulated by the TGF- $\beta$ signaling 
pathway, responding to a large number of extracellular stimuli. Best known for control of cell growth and differentiation, TGF- $\beta$ signaling functions primarily as a growth inhibitory pathway, which controls cell growth and differentiation via its interactions with Smad proteins (Massague, 2000). The two receptor Smads investigated in this study, Smad 2 and Smad 3, are activated via phosphorylation by TGF- $\beta$ receptor kinases upon binding of TGF- $\beta$, activins, or nodals to the receptor (Lee and Bae, 2001). The phosphorylation of a receptor Smad results in its nuclear translocation, via formation of a heterocomplex with common Smad 4. In carcinoma epithelial cells, induction of TGF- $\beta$ functions as a potent growth inhibitor, and the mechanism is characterized by its ability to mediate cell cycle arrest and induce apoptosis (Coffey et al., 1988). In addition, previous studies have also shown that exposure of endothelial cells to hypoxic conditions induces the expression and activation of TGF- $\beta$, followed by an induction of DNA binding Smad proteins (Zhang et al., 2003).

The survival strategy of hibernation requires a profound global suppression of basal metabolic rates, as well as selective activation of various stress-responsive pathways to achieve particular results. Because of the anti-growth properties of TGF- $\beta$ signaling, I hypothesized that a potential increase in Smad transcriptional activities would take place as a mechanism for cell cycle arrest during torpor. The present chapter examined the regulation pattern of Smad 2 and Smad 3 transcription factors, as well as selected downstream target genes, over the torpor-arousal cycle in liver and skeletal muscle of hibernating ground squirrels.

\subsubsection{Translational and post-translational regulation of Smads}


The transduction of the TGF- $\beta$ signal into a cellular response requires the activation of the transcriptional activity of receptor Smads; this is accomplished through phosphorylation of Smad 2 or Smad 3 in their C-terminal SSXS regions, followed by their nuclear translocation in a complex with Smad 4. Results from Western immunoblotting show tissue-specific responses of Smad regulation between liver and skeletal muscle. Total expression levels of Smad 2 and Smad 3 were unchanged in skeletal muscle over the torpor-arousal cycle and showed only selective change in liver, displaying significant reductions in total Smad 2 protein level in LT and IA, and total Smad 3 protein in EN (FIG. 5.3, 5.4). Reduced total Smad levels could potentially be linked to increased expression of Smurf (Smad ubiquitination regulatory factor 2), an E3 ubiquitin ligase that tags Smad proteins for degradation by the proteasome. When expressed at high concentrations, Smurf 2 has previously been shown to suppress Smad 2 levels but had minimal effects on Smad 3 (Zhang et al., 2001). However, protein levels of Smurf 2 were largely unchanged during hibernation, with selected small changes mainly in muscle and a reduction in liver Smurf 2 only in IA (FIG. 5.5). Hence, there was no consistent correlation between Smad 2 and Smurf 2 expression, suggesting that the decrease in Smad 2 expression in liver was likely independent of Smurf 2 mediated proteasome degradation.

Changes in total protein expression are indicators of regulation at the transcriptional or translational level, but the primary determinant of rapid change in Smad activity in cells is the phosphorylation state of R-Smads. Interestingly, although Smad 2 and Smad 3 respond to nearly identical stimuli, differential phosphorylation patterns were observed for both proteins. The phosphorylation state of Smad 2 at the C-terminal Ser- 
465/ Ser-467 site remained unchanged throughout the torpor-arousal cycle in both liver and muscle (FIG. 5.6). Furthermore, the nuclear content of p-Smad 2 was also unchanged during LT (FIG. 5.8) and together these data suggest that Smad 2 has little or no involvement in gene regulation over the torpor-arousal cycle.

Unlike Smad 2, phosphorylation levels of Smad 3 at the C-terminal Ser-423/ Ser425 site displayed a tissue specific regulation pattern. The relative content of phosphorylated Smad 3 increased by 1.4-1.6 fold in liver during the two torpor stages, but was elevated only during entrance into torpor in the skeletal muscle (FIG. 5.7). To determine if this tissue specific activation of Smad 3 was also mirrored by changes in the Smad 4 partner protein, total Smad 4 expression was analyzed (FIG. 5.2). Total Smad 4 was elevated at both torpor stages in liver, matching the increases in phosphorylation of Smad 3 at these stages but total Smad 4 levels in skeletal muscle were unchanged over the torpor-arousal cycle. The increase in phosphorylated Smad 3 and total levels of Smad 4 in liver during torpor suggest that translocation of Smad 3 to the nucleus should also increase during torpor, since formation of the heterocomplex Smad3:Smad4 requires the interaction of phosphorylated SSXS motif of Smad 3 with the basic pockets of the MH2 domain in Smad 4 (Massagué, 2005). Indeed, levels of nuclear p-Smad 3 were elevated by nearly 2.5 -fold during LT and correlated with a comparable increase in the nuclear content of Smad 4 (FIG.5.8). This type of response has also been observed in other forms of metabolic stress; for example, elevated phosphorylation and nuclear translocation of Smad 3 occurred in response to hypoxia (Zhang et al., 2003). A strong increase in the nuclear content of p-Smad 3 was also observed in skeletal muscle (FIG.5.8), although total p-Smad 3 was unchanged in LT (FIG. 5.7). Previous studies have shown that Smad 
4 is often equally distributed between the nucleus and cytoplasm and undergoes continuous nucleocytoplasmic shuttling. Upon stimulation with a TGF- $\beta$ signal, nuclear accumulation of Smad 4 can occur within 30 minutes, presumably due to increased of RSmad shuttling (Pierreux et al., 2000). The stable expression of Smad 4 observed in the skeletal muscle would suggest that although active shuttling of Smad 4 is likely to take place, the lack of Smad 4 nuclear accumulation may be an indicator for lack of TGF- $\beta$ signals in the skeletal muscle during torpor (Schmierer and Hill, 2007). The increased nuclear content of active Smad 3 in both tissues during torpor suggests an important role for Smad 3 mediated gene regulation at this stage. Overall, the results suggest that activation of Smad signaling in hibernation is only evident in the liver; however, only Smad 3 showed patterns of activation, which involves the phosphorylation and subsequent nuclear accumulation of Smad proteins, leading to changes in transcriptional activity.

\subsubsection{Transcriptional and sequence analysis of Smads}

The regulation of cellular processes requires the action of protein molecules, whose expression and function ultimately governs the biological activities. However, regulation of proteins often takes place at the transcriptional level, where changes in mRNA levels functions to regulate the availabilities of protein products. As demonstrated above, Smad action in hibernation clearly depends on phosphorylation and nuclear translocation but a full understanding of the role of Smads in hibernation also requires knowledge of Smad gene expression responses over torpor-arousal. Expression of Smad 2, Smad 3 and Smad 4 genes was analyzed using RT-PCR. As seen in FIG.5.9, the 
expression of Smad 2 increased during the arousal phases of hibernation in the liver, while decreasing during the LT and EA phase in the skeletal muscle. By contrast, Smad 3 transcript levels increased during EA in liver and EN in skeletal muscle (FIG. 5.12). Neither Smad 2 nor Smad 3 showed good correlation between changes in mRNA levels and protein levels. This type of discrepancy is often observed, although not always understood. Due to the multi-step process that takes place between mRNA transcription and functional protein products, potential RNA or protein degradation could function to influence the gene expression (Wang, 2008). In FIG. 5.15, the expression level of Smad 4 showed no changes over the torpor-arousal cycle in both liver and skeletal muscle; this correlated with the previous observation of stable Smad 4 protein expression in skeletal muscle, but not liver. The stable expression of Smad 4 at the mRNA and protein level in skeletal muscle suggests that Smad 4 can be viewed as a housekeeping gene, where basal expression is maintained throughout the hibernation cycle.

Using sequence information derived from PCR amplicons, partial amino acid sequences of ground squirrel Smad 2, Smad 3, and Smad 4 were predicted (FIG. 5.10, 5.13, and 5.16) and homologies with the proteins of non-hibernating mammals (mouse, rat, and human) were evaluated were analyzed (FIG. 5.11, 5.14, and 5.17. All three Smad proteins displayed high homology as compared with the three other species. Indeed, ground squirrel Smad 3 was identical with the rat and human sequences, and showed $99.8 \%$ identify with mouse Smad 3. The high degree of amino acid similarities observed indicates that the structure and function of Smad proteins are well conserved in mammals. Furthermore, the homology analysis gave no evidence of hibernator-specific changes in amino acid sequence such as have been found for other proteins, for example 
fatty acid binding protein (Hittel and Storey, 2001).

\subsubsection{Nuclear Smad 3 DNA activity}

The immunoblotting data showed that Smad 3, but not Smad 2, displayed increased levels of phosphorylation and nuclear inclusion during hibernation. However, analysis also showed that Smad 3 displayed tissue-specific activation during the torpor stages of hibernation, with increased in Smad 3 phosphorylation only evident in liver. To further explore Smad 3 regulation, an EMSA analysis was used and showed positive interactions between nuclear extracts of liver with biotin labelled double stranded DNA probes containing the SBE (FIG. 5.18). The observed shift in lane 3 due to protein binding to the SBE appeared to be mediated by Smad 3, as the band disappeared in the presence of Smad 3 monoclonal antibody (lane 4). The SBE probe utilized in this experiment has also previously been shown to bind to Smad proteins in human cell lines, suggesting that SBE sequence is conserved in the ground squirrel (Qiao et al., 2009). Furthermore, the DNA binding activity of Smad 3 was elevated by $\sim 1.6$-fold during LT in the liver, but remained unchanged in the skeletal muscle (FIG. 5.19). This increase in DNA binding by Smad 3 in the liver correlated with the increase in Smad 3 phosphorylation and nuclear p-Smad 3 content. Overall, then, all of these pieces of evidence parallel those seen for Smad 3 responses to hypoxia in human cell lines (Zhang et al., 2003), and all indicate that Smad 3 increases its transcriptional activity during torpor and stimulates the expression of various gene targets under its control.

\subsubsection{Discrepancy in regulation of liver and skeletal muscle}

Although liver and skeletal muscle are physiologically distinct in terms of their 
biological functions, it remains unexplained why Smad 3 DNA binding activity did not change in nuclear extracts of skeletal muscle (FIG. 5.19) despite the increase in nuclear p-Smad 3 content in muscle (FIG. 5.8). It is possible that binding of Smad 3 to DNA requires the co-presence of nuclear Smad 4, and that the lack of increase in nuclear Smad 4 in skeletal muscle (FIG. 5.8) could function to limit Smad 3 DNA binding. This was shown in FIG. 5.20, where co-immunoprecipitated expression of skeletal muscle Smad 4 was measured in samples precipitated for Smad 3. When comparing the phosphorylated levels of Smad 3, although p-Smad 3 was readily detected, the amount of Smad 4 observed in skeletal muscle was less than that observed in the liver. This suggests that an increase in Smad 3 phosphorylation alone might not be enough to elicit a cellular response, and a co-increase in Smad 4 nuclear expression (as observed in liver) might be required to induce an increase in Smad 3 transcriptional activity.

\subsubsection{Expression of p15INK4b and p21CIP1 in response to Smad 3 activation}

One of the major functions of Smad transcription activity is to induce cell cycle arrest via the expression of the cell cycle inhibitors, p15INK4b and p21CIP1 (Feng et al., 2000; Seoane et al., 2001). Although p15INK4b and p21CIP1 are two different classes of cell cycle inhibitors, they both target cyclin dependent kinases (Cdk) in the early phases of the cell cycle (Nakayama and Nakayama, 1998). The INK4 family of cell cycle inhibitors target Cdk proteins directly, causing an allosteric change in Cdk structure that inhibits their interactions with cyclin proteins. p15INK4b primarily targets Cdk 4 and Cdk 6, thus functioning as a specific inhibitor for cyclin D-dependent kinases, stalling the cell cycle at the $\mathrm{G}_{1}$ phase (Pavletich, 1999; McConnell et al., 1999). Meanwhile, the CIP 
family of cell cycle inhibitors functions by binding with a variety of Cdk:cyclin complexes. p21CIP1 has been shown to induce cell cycle arrest at the $\mathrm{G}_{2} / \mathrm{M}$ checkpoint via interactions with the Cdk 1/ cyclin B complex, but has also been shown to regulate the $\mathrm{G}_{1} / \mathrm{S}$ checkpoint through its interaction with DNA polymerase loading clamp PCNA (proliferating cell nuclear antigen) (McConnell et al., 1999; Cmielová and Rezáčová, 2011).

Shown in FIG.5.21, the relative mRNA expression of both $p 15 I N K 4 b$ and p21CIP1 in liver was elevated during both torpor stages of hibernation. This upregulation correlates with the strong activation of Smad 3 transcription factor activity seen in this tissue, suggesting that the upregulation of the cell cycle inhibitors was most likely to be Smad 3-mediated. Interestingly, in skeletal muscle expression of $p 21 C I P 1$ was also elevated during LT and EA, but expression of $p 15 I N K 4 b$ remained stable (FIG.5.22). The predicted sequence of the two inhibitor proteins derived from amplified PCR products are shown in FIG. 5.23-5.26. Although both p15INK4b and p21CIP1 show moderately high degree of conservation compared to the three sequences from nonhibernating mammalian, the level of homology was less than those observed for Smad proteins. The predicted protein sequence of p15INK4b showed a homology in the range of $86.3 \%-87.0 \%$, slightly higher than p $21 \mathrm{CIP} 1$ with a range of $78.1 \%-82.2 \%$.

One of the major differences between liver and skeletal muscle cells is that myocytes are considered to be terminally differentiated, and do not undergo active cell divisions whereas hepatocytes do proliferate (Montarras et al., 1989; Loyer et al., 2012). As shown in this study, an activation of Smad 3 mediated transcription of target genes in 
the liver is likely to function as an anti-growth signal, aimed at reducing cell division during torpor through the induction of cell cycle inhibitors. This could possibly explain why tissue specific activation of Smad 3 transcriptional activity was observed in the liver, but not skeletal muscle. However, the upregulation of $p 21 C I P 1$ in skeletal muscle suggests that although muscle cells are terminally differentiated, components of cell cycle regulators are still present. The functions of these inhibitors outside of their canonical role in cell cycle regulation are relatively unknown. However, recent studies have also suggested that $\mathrm{p} 15 \mathrm{INK} 4 \mathrm{~b}$ could function in an alternate signal transduction cascade as shown in hematopoietic cells; the induced expression of p15INK4b have resulted in activation of MEK/ERK signaling, and increasing the expression of GATA-1 and GATA2 transcription factors (Humeniuk et al., 2013; Wolff and Bies, 2013).

\subsubsection{Smad mediate expression of microRNA}

Next to $p 15 I N K 4 b$ and $p 21 C I P 1$, Smad transcriptional activity has also been previously shown to regulate the expression of a number of microRNAs. Similar to the mechanism previously described, activation of receptor Smads via TGF- $\beta$ stimulation results in the nuclear activity of Smad towards SBE in the pri-microRNA promoters (Davi-Dusenbery and Hata, 2011; Davis et al., 2008). MicroRNA are short strands of non-coding RNA that are 18-25 nucleotide in length, they primarily function to suppress gene expression through binding with target mRNA at the 3' untranslated region with either full or partial complementarity (Leung and Sharp, 2010). Expression of mature microRNA 21, 29b, and 155 in liver were all elevated at one or more point of the torporarousal phase (FIG. 5.27), with miR-21 and miR-155 upregulated in entry and early 
phases of torpor. Notably, miR-29b was observed to be upregulated in both torpor stages, correlated with the activation pattern of Smad 3 in this tissue, as well with the other two Smad regulated genes $p 15 I N K 4 b$ and $p 21 C I P 1$. In skeletal muscle, however, minimal changes in microRNA expression were observed with only miR-29b and miR-155 elevated during ET and IA respectively (FIG. 5.28).

MicroRNA-21 is best characterized in its role in cancer cells, where it has been reported to be upregulated in a number of malignancies such as glioma, breast cancer, ovarian cancer, and colorectal cancer (Chan et al., 2005; Volinia et al., 2006; Iorio et al., 2007). While a large number of potential miR-21 targets have been reported (mostly based on microarray screening technology), only a small subset of these targets have been validated. One of these genes is Smad 7, an inhibitory Smad protein that functions as a negative regulator of TGF- $\beta$. Recent studies have shown that upregulation of $m i R-21$ expression enhanced TGF- $\beta$ signaling, thereby creating a possible mechanism that forms a feedback cycle, mediated through suppression of inhibitory Smad 7 expression (Marquez et al., 2010). This would suggest that the increased in $m i R-21$ expression in liver during the early torpor stages could function as a positive feedback to stimulate Smad 3 activation throughout the torpor stages, by suppressing the levels of Smad 7 expression.

MicroRNA-155 is known to be a direct transcriptional target of Smad, with RhoA mRNA transcripts being one of its best characterized inhibitory targets (Kong et al., 2008). RhoA is a member of the Rho GTPase, which functions to regulate a wide range of cellular processes such as cytoskeleton dynamics, cell cycle progression, and cellular 
transformation (Zhang et al., 2009). More importantly, it has previously been shown that RhoA is a crucial regulator of the $\mathrm{G}_{1} \rightarrow \mathrm{S}$ transition phase of the cell cycle; RhoA positively regulates cell cycle progression via effects on cell cycle inhibitors, and suppression of RhoA has previously been shown to induce cell cycle arrest (Zhang et al., 2009). Although the expression levels of RhoA were not measured, the upregulation of miR-155 in liver could suggest that Smad mediated cell cycle arrest functions by more than a single mechanism (not just induction of $p 15 I N K 4 b$ and $p 21 C I P 1$ ), and that control of RhoA via microRNA expression could also play a major role in cell cycle arrest.

Like the other two microRNAs discussed, miR-29b is also best known for its role in cancer malignancies and is involved in a large number of cellular processes such as proliferation, differentiation, and apoptosis (Kriegel et al., 2012). One of the best characterized targets of miR-29b is $\mathrm{p} 85 \alpha$, the catalytic subunit of PI3K, a major component of the insulin signaling pathway (Park et al., 2009). The upregulation of miR$29 \mathrm{~b}$ in both torpor stages during hibernation suggests that it could function to regulate the insulin signaling pathway during torpor, via its suppression of $\mathrm{p} 85 \alpha$ translation. Indeed, previous studies have shown that the phosphorylation state of the Akt kinase, a downstream target of PI3K, was significantly reduced during both torpor stages in the liver of ground squirrels (McMullen and Hallenbeck, 2010). This could suggest that Smad mediated microRNA expression functions to regulate not only cell cycle progression, but also other energy dependent cellular processes such as insulin signaling in the liver during hibernation.

\subsection{Conclusion}


The present chapter suggests an important role for the Smad family of transcription factors in regulation of the torpor-arousal cycle during ground squirrel hibernation. Smad 3 specifically, but not Smad 2, is selectively activated in the liver in response to hibernation. The activation of Smad 3 transcriptional activity led to increased expression of cell cycle inhibitors, as well as upregulation of three microRNA species that are involved in the negative regulation of metabolic functions. Overall, the findings reported here establish TGF- $\beta$ mediated Smad signaling as one of the primary antiproliferatory responses activated during mammalian metabolic rate depression. 


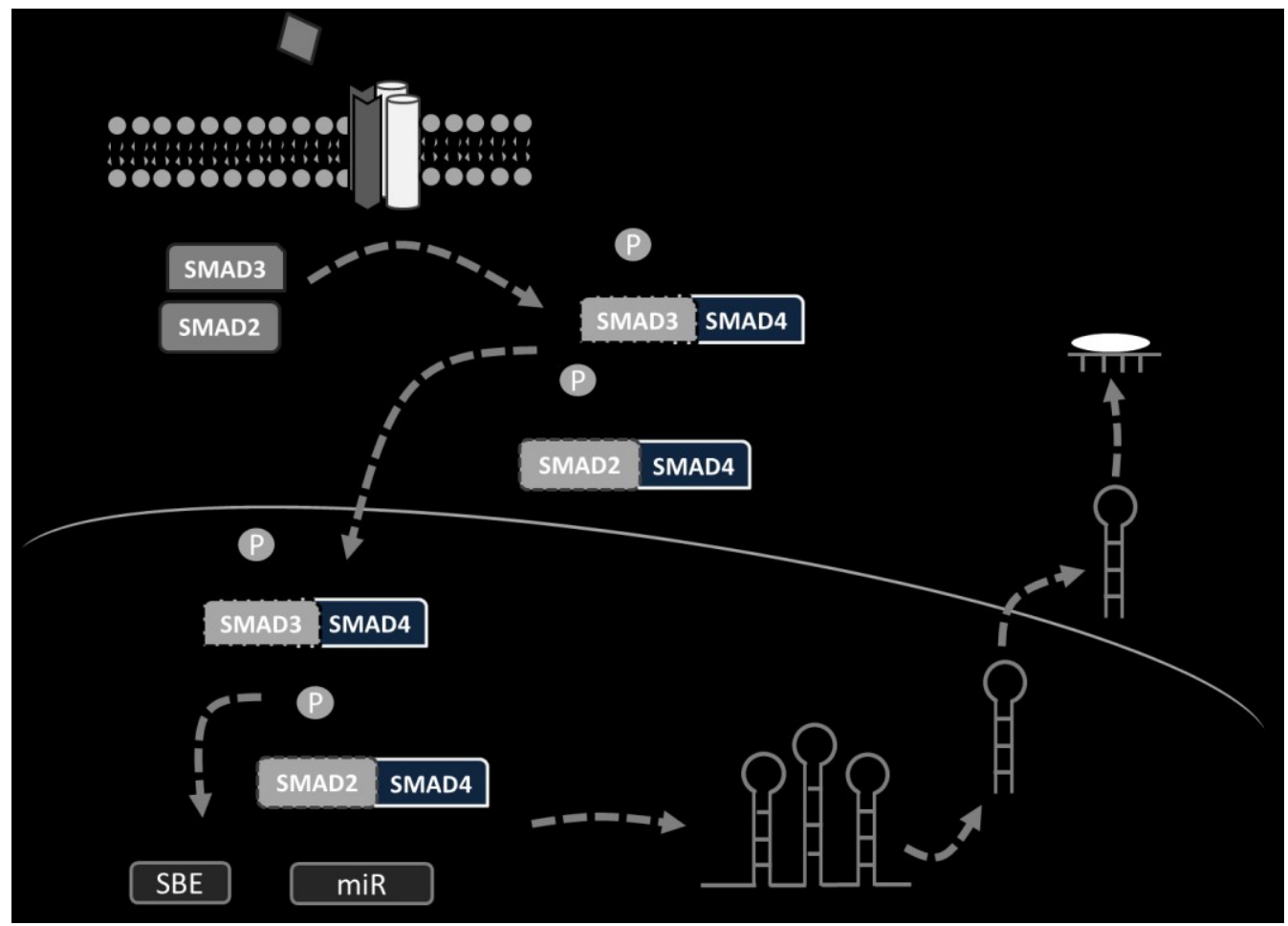

\section{FIG 5.1 Mechanism of Smad mediated transcriptional response.}

Activation of membrane receptor protein kinases (Type I and Type II through

dimerization) is achieved in response to extracellular stimuli such as TGF- $\beta$ and results in the phosphorylation of R-Smads (Smad 2 and 3). The phosphorylation of R-Smads leads to assembly of a multisubunit complex with common Smad (Smad 4), resulting in the nuclear translocation of the Smad transcriptional complex. The nuclear Smad transcription complex interacts with Smad binding element (SBE) motifs on genes, leading to transcriptional activation of downstream targets such as microRNA transcripts. 


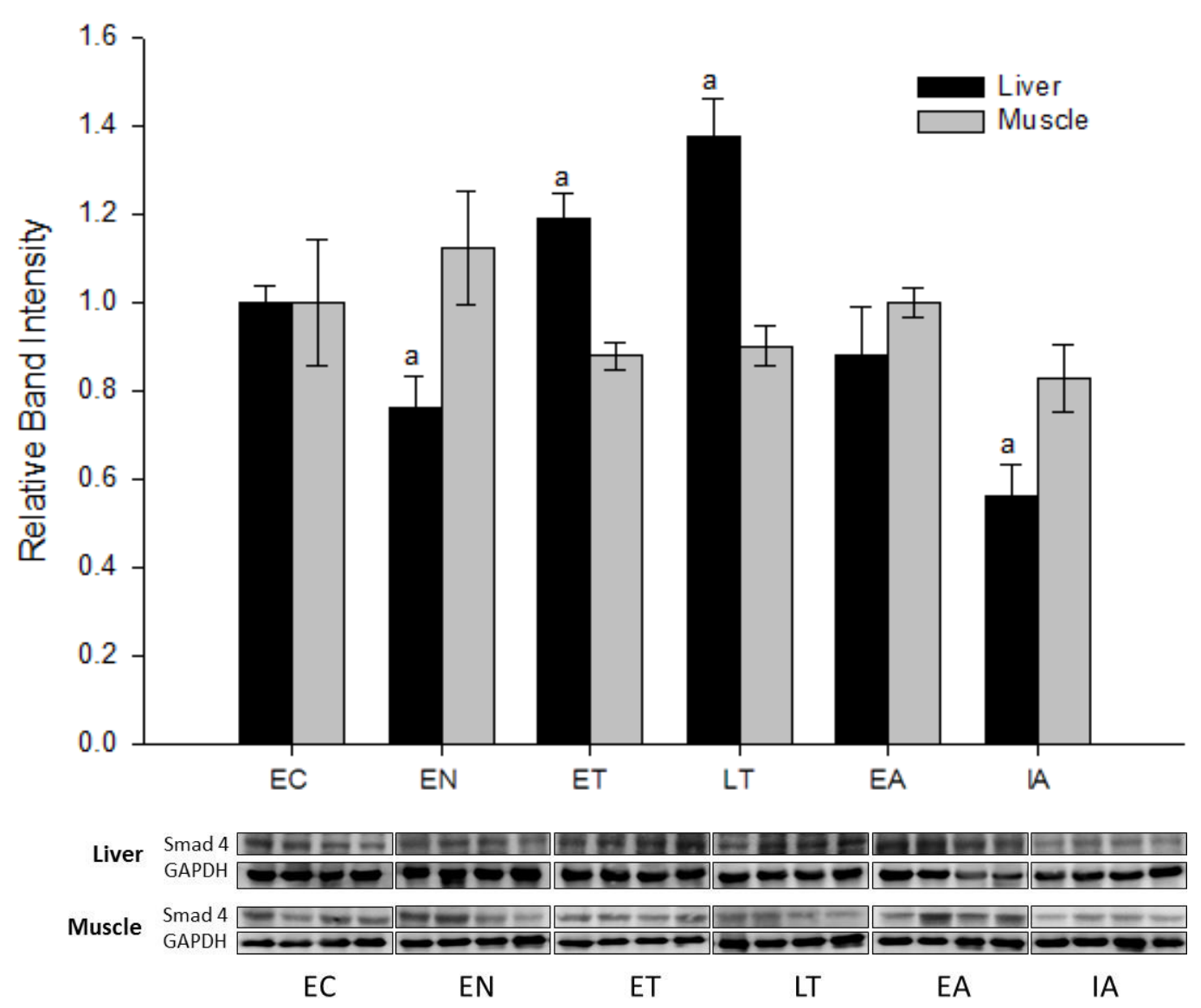

FIG. 5.2. Regulation pattern of Smad 4 protein in liver and skeletal muscle of $I$. tridecemlineatus as determined by Western immunoblotting.

Relative expression of SMAD 4 protein over the torpor-arousal cycle in liver and skeletal muscle of 13-lined ground squirrels. Western blot bands of SMAD 4 were detected at $\sim 70 \mathrm{kDa}$ and the housekeeping protein GAPDH was detected at $\sim 32 \mathrm{kDa}$.

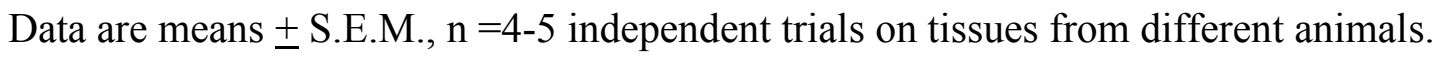
EC, Euthermic control; EN, early entrance into torpor; ET, early torpor; LT, late torpor; EA, early arousal; IA, interbout arousal. a-Significantly different from the corresponding EC values $(\mathrm{P}<0.05$, ANOVA with post hoc Holm-Sidak test). 

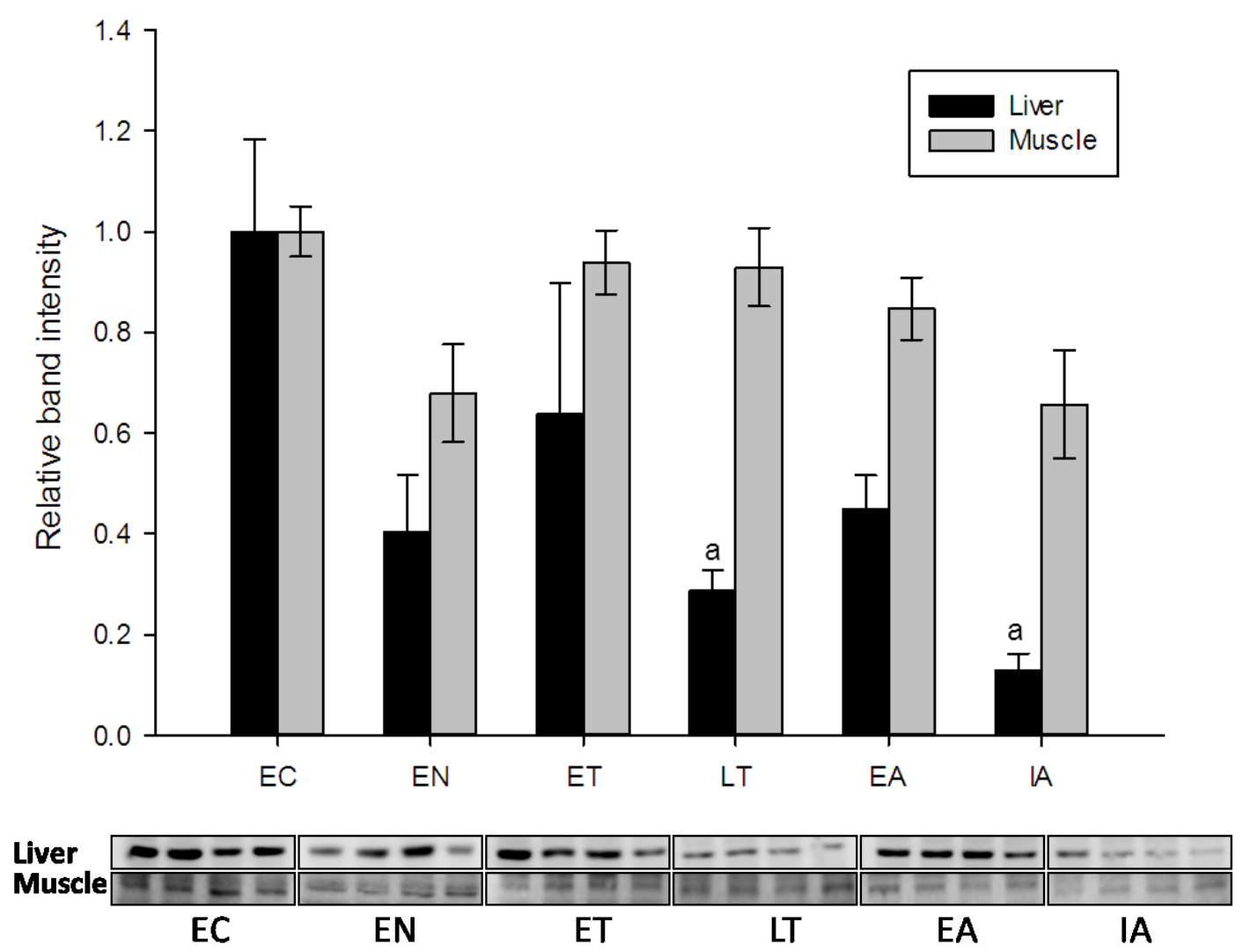

FIG. 5.3. Regulation pattern of Smad 2 protein in liver and skeletal muscle of $I$. tridecemlineatus as determined by Western immunoblotting.

Relative expression of SMAD 2 protein over the torpor-arousal cycle in liver and skeletal muscle of 13-lined ground squirrels. Western blot bands of SMAD 2 were detected at $\sim 60 \mathrm{kDa}$. Data are means \pm S.E.M., $\mathrm{n}=4-5$ independent trials on tissues from different animals. a-Significantly different from corresponding EC values $(\mathrm{P}<0.05$, ANOVA with post hoc Holm-Sidak test). 


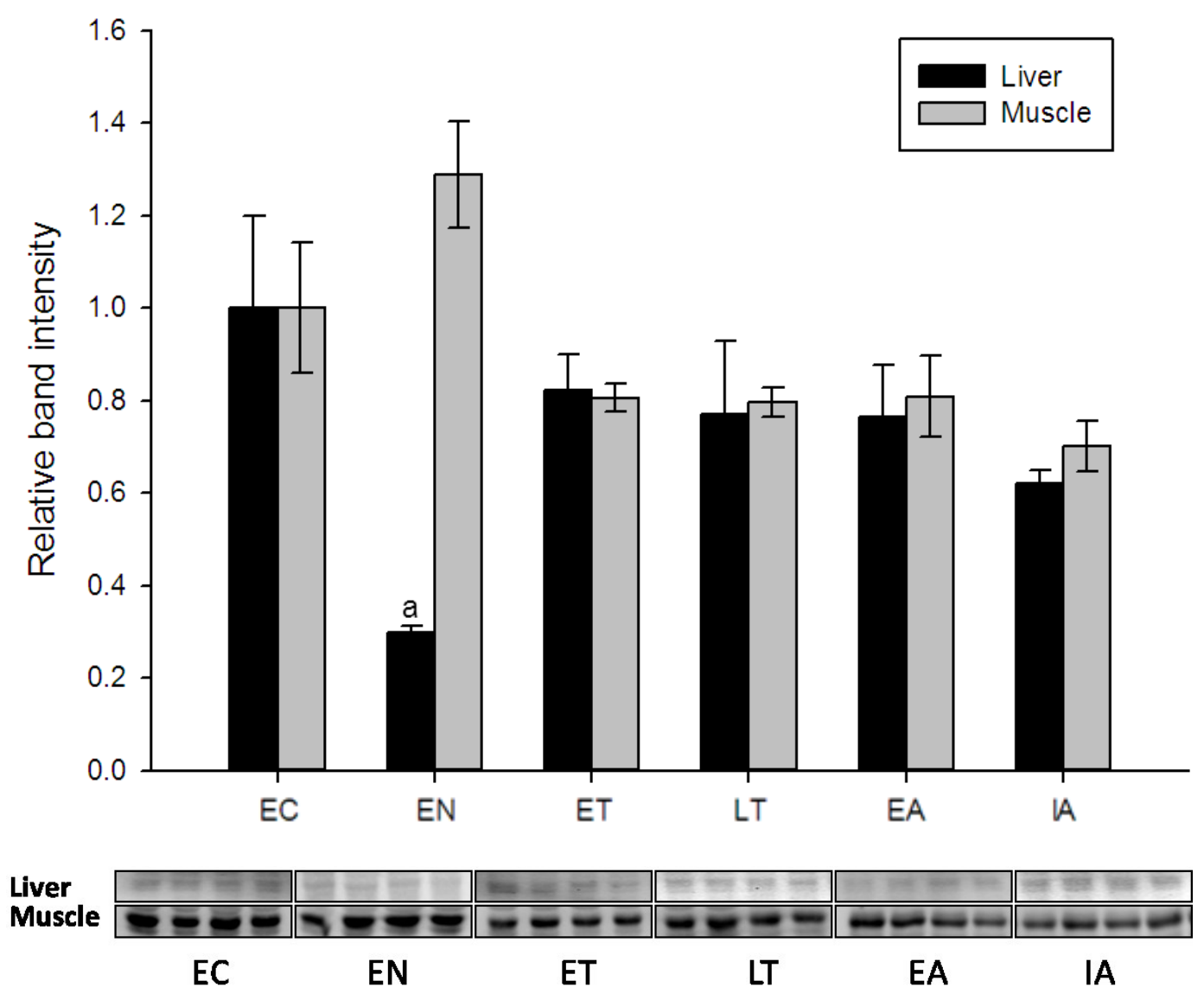

FIG. 5.4. Regulation pattern of Smad 3 protein in liver and skeletal muscle of $I$. tridecemlineatus as determined by Western immunoblotting.

Relative expression of SMAD 3 protein over the torpor-arousal cycle in the liver and skeletal muscle of 13-lined ground squirrels. Western blot bands of SMAD 3 were detected at $\sim 52 \mathrm{kDa}$. Data are means \pm S.E.M., $\mathrm{n}=4-5$ independent trials on tissues from different animals. a-Significantly different from corresponding EC values $(\mathrm{P}<0.05$, ANOVA with post hoc Holm-Sidak test). 

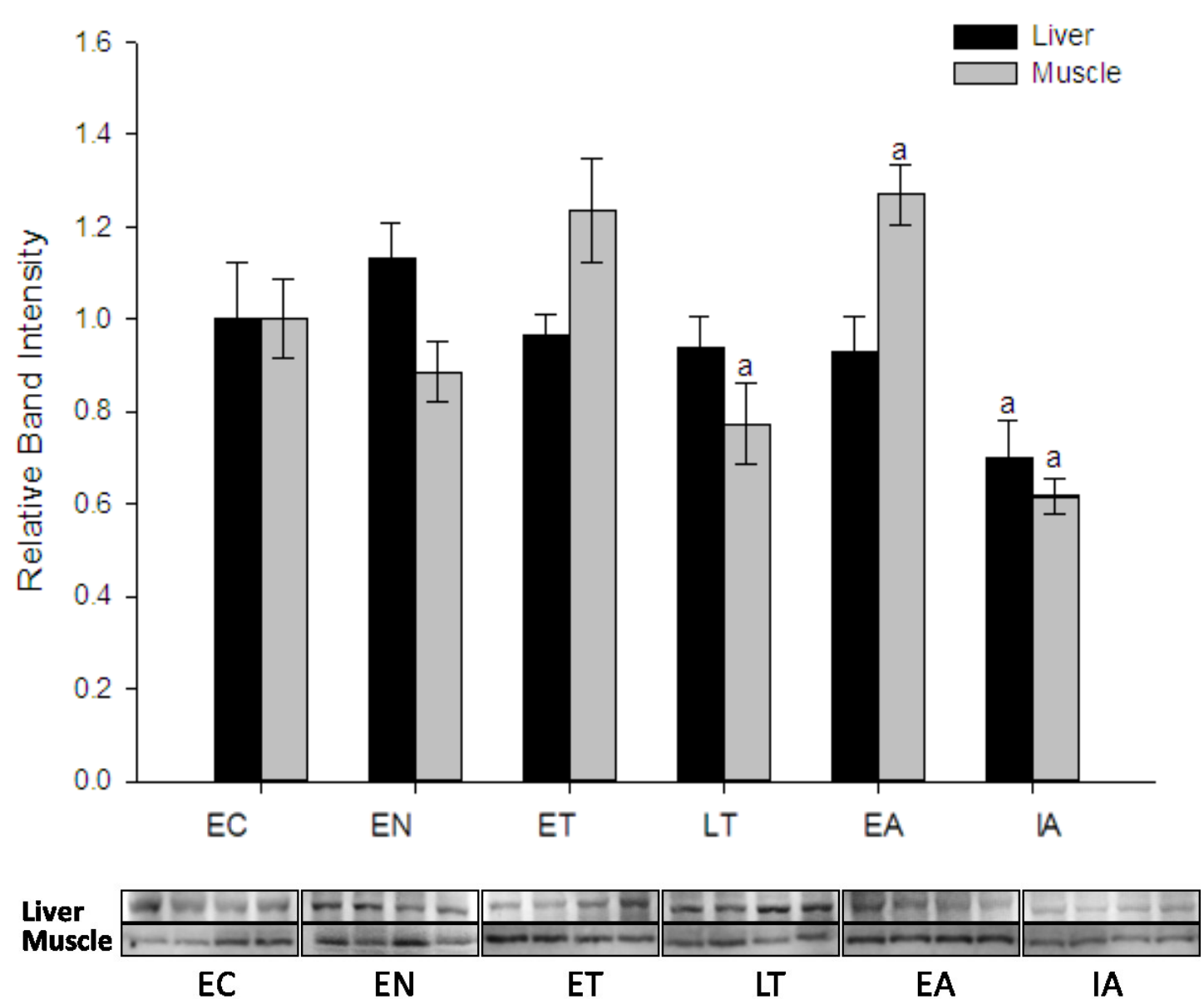

FIG. 5.5. Regulation pattern of Smurf 2 protein in liver and skeletal muscle of $I$. tridecemlineatus as determined by Western immunoblotting.

Relative protein levels of SMURF2 protein over the torpor-arousal cycle in liver and skeletal muscle of 13-lined ground squirrels. Western blot bands of SMURF 2 were detected at $\sim 90 \mathrm{kDa}$. Data are means \pm S.E.M., $\mathrm{n}=4-5$ independent trials on tissues from different animals. a-Significantly different from corresponding EC values $(\mathrm{P}<0.05$, ANOVA with post hoc Holm-Sidak test). 

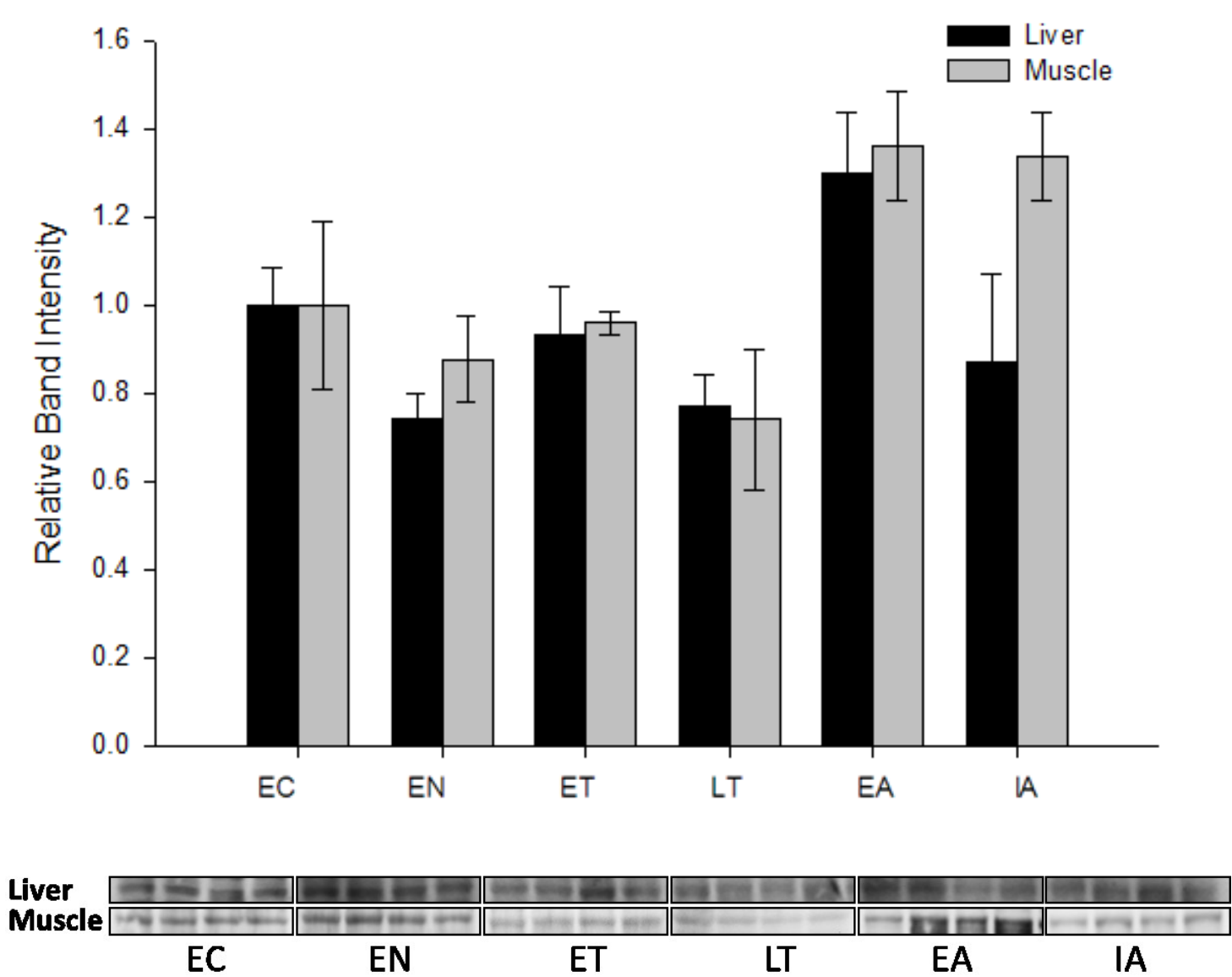

FIG. 5.6. Regulation pattern of phosphorylated Smad 2 protein (Ser 465/467) in liver and skeletal muscle of I. tridecemlineatus as determined by immunoblotting.

Relative protein levels of phospho-SMAD2 ${ }^{\text {Ser465/467 }}$ over the torpor-arousal cycle in liver and skeletal muscle of 13-lined ground squirrels. Western blot bands of phosphoSMAD2 ${ }^{\operatorname{Ser} 465 / 467}$ were detected at $\sim 60 \mathrm{kDa}$. Data are means \pm S.E.M., $\mathrm{n}=4-5$ independent trials on tissues from different animals. 

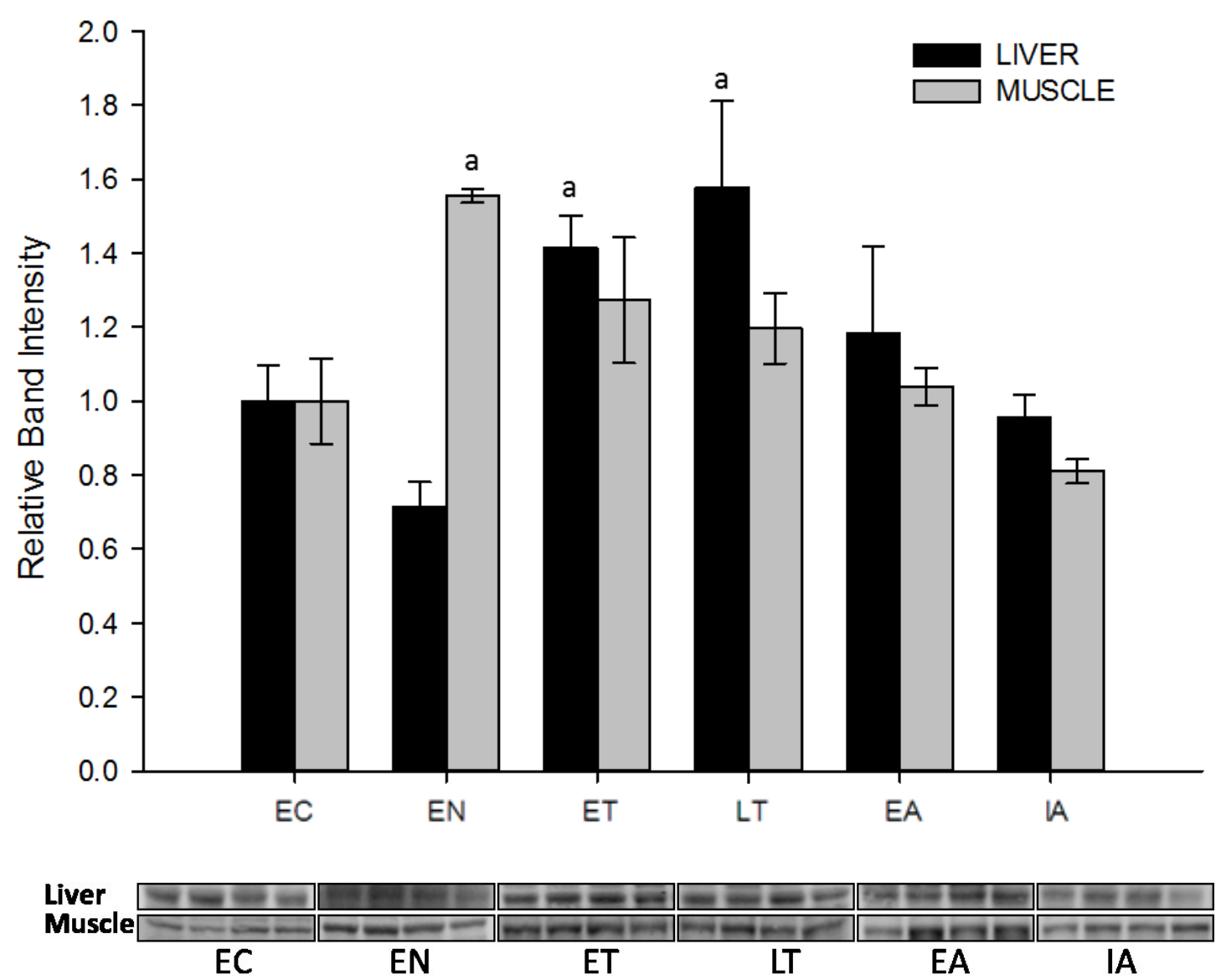

FIG. 5.7. Regulation pattern of phosphorylated Smad 3 (Ser 423/425) protein in liver and skeletal muscle of I. tridecemlineatus as determined by immunoblotting.

Relative protein levels of phospho-SMAD3 ${ }^{\text {Ser423/425 }}$ over the torpor-arousal cycle in liver and skeletal muscle of 13-lined ground squirrels. Western blot bands phosphoSMAD3 ${ }^{\text {Ser423/425 }}$ were detected at $\sim 52 \mathrm{kDa}$. Data are means \pm S.E.M., $\mathrm{n}=4-5$ independent trials on tissues from different animals. a-Significantly different from corresponding EC values $(\mathrm{P}<0.05$, ANOVA with post hoc Holm-Sidak test). 


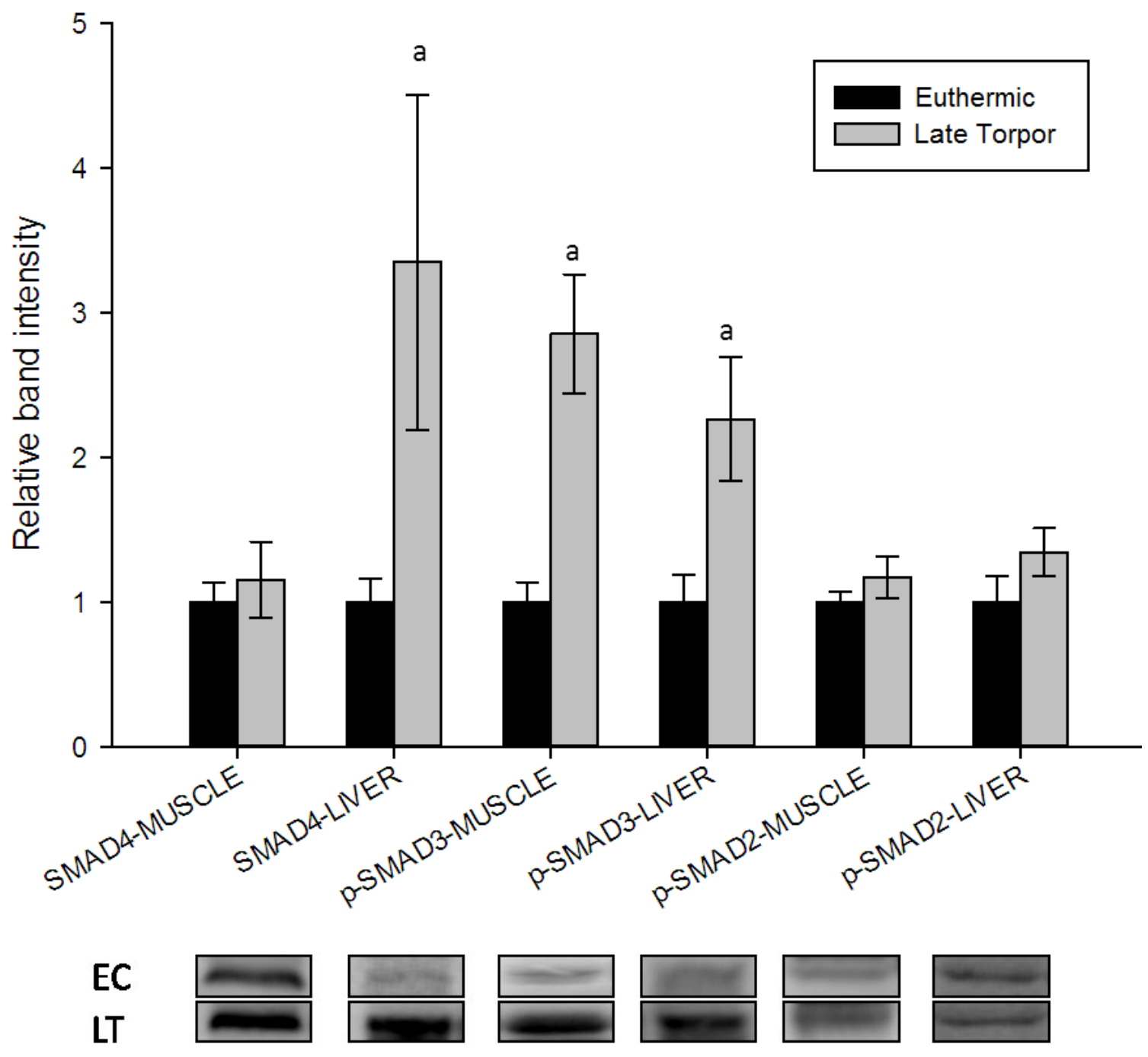

FIG. 5.8. Nuclear localization of SMAD proteins in liver and skeletal muscle of $I$. tridecemlineatus as determined by immunoblotting.

Relative nuclear protein expression of SMAD 4, phospho-SMAD3 ${ }^{\text {Ser423/425, and }}$

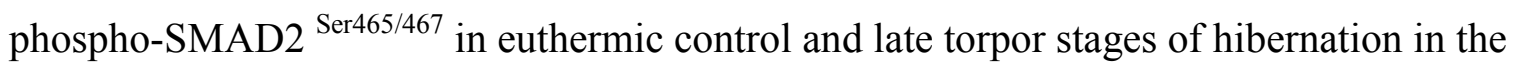
liver and skeletal muscle of 13-lined ground squirrels. Representative data are means \pm S.E.M., $\mathrm{n}=4-5$ independent trials on tissues from different animals. a-Significantly different from corresponding euthermic values $(\mathrm{P}<0.05$, Student's t-test). 

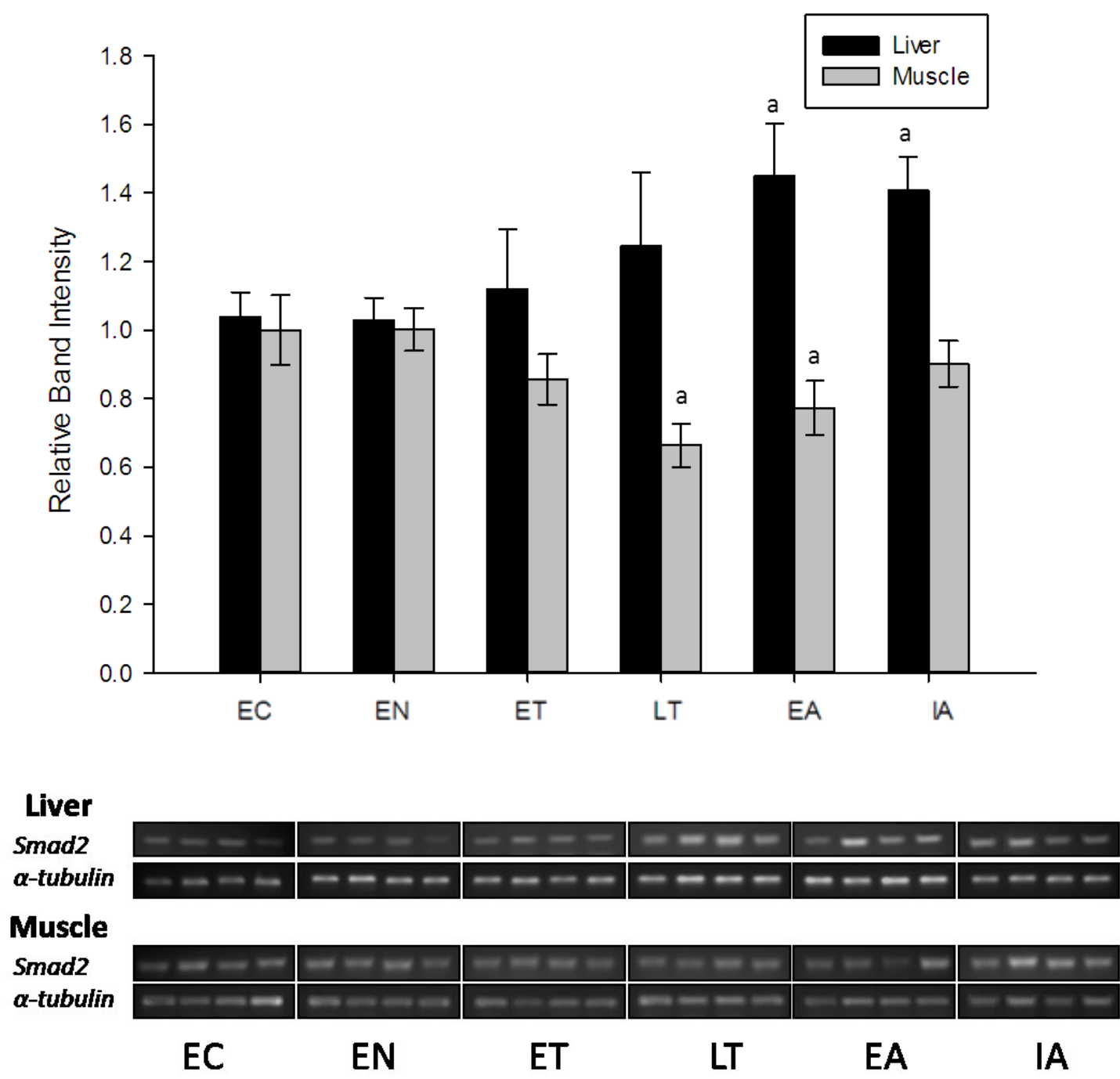

FIG. 5.9. Transcriptional regulation of $S \mathrm{mad} 2$ in liver and skeletal muscle of $I$. tridecemlineatus as determined by RT-PCR.

Smad2 mRNA transcript levels in the liver and muscle of 13-lined ground squirrels over the stages of the torpor-arousal cycle. SyBR green stained agarose gels are shown for Smad2 genes and for the housekeeping gene, $\alpha$-tubulin. Data are means \pm S.E.M., $\mathrm{n}=4$ independent trials on tissues from different animals. a-Significantly different from corresponding euthermic values $(\mathrm{P}<0.05$, ANOVA with post hoc HolmSidak test). 
GCAATACCCTCAAATTGTATACTAAATGTGTTACCATACCAAGCACTTGCTCTGAAATTT $\begin{array}{llllllllllllllllllll}W & G & \mathrm{~L} & \mathrm{~S} & \mathrm{~T} & \mathrm{P} & \mathrm{N} & \mathrm{T} & \mathrm{I} & \mathrm{D} & \mathrm{Q} & \mathrm{W} & \mathrm{D} & \mathrm{T} & \mathrm{T} & \mathrm{G} & \mathrm{L} & \mathrm{Y} & \mathrm{S} & \mathrm{F}\end{array}$ CTGAACAAACCAGATCTCTTGATGGTCGCCTTCAAGTTTCTCATCGAAAAGGGTTACCAC $\begin{array}{llllllllllllllllllllllllllllllll}S & E & Q & T & R & S & L & D & G & R & L & Q & V & S & H & R & K & G & L & P\end{array}$ ATGTTATCTATtGCCGGTTATGGCGCTGGCCTGATCTTCACAGTCATCATGAACTCAAGG ACCACTATCAGAGAGTCGAGACACCAGTTTTGCCTCCGGTATTAATGCCACGACACACTG $\begin{array}{lllllllllllllllllllllll}\mathrm{Y} & \mathrm{H} & \mathrm{Y} & \mathrm{Q} & \mathrm{R} & \mathrm{V} & \mathrm{E} & \mathrm{T} & \mathrm{P} & \mathrm{V} & \mathrm{L} & \mathrm{P} & \mathrm{P} & \mathrm{V} & \mathrm{L} & \mathrm{M} & \mathrm{P} & \mathrm{R} & \mathrm{H} & \mathrm{T}\end{array}$

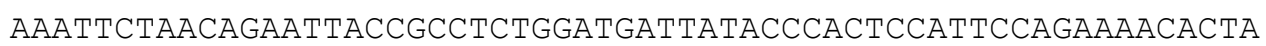
$\begin{array}{llllllllllllllllllllllllllll}\mathrm{E} & \mathrm{I} & \mathrm{L} & \mathrm{T} & \mathrm{E} & \mathrm{L} & \mathrm{P} & \mathrm{P} & \mathrm{L} & \mathrm{D} & \mathrm{D} & \mathrm{Y} & \mathrm{T} & \mathrm{H} & \mathrm{S} & \mathrm{I} & \mathrm{P} & \mathrm{E} & \mathrm{N} & \mathrm{T}\end{array}$ ACTTCCCAGCGgGAATTGAGCCACAGAgTAATTATATTCCAGAAACACCACCTCCTGGAT $\begin{array}{lllllllllllllllllllllllllll}\mathrm{N} & \mathrm{F} & \mathrm{P} & \mathrm{A} & \mathrm{G} & \mathrm{I} & \mathrm{E} & \mathrm{P} & \mathrm{Q} & \mathrm{S} & \mathrm{N} & \mathrm{Y} & \mathrm{I} & \mathrm{P} & \mathrm{E} & \mathrm{T} & \mathrm{P} & \mathrm{P} & \mathrm{P} & \mathrm{G}\end{array}$ AтATCATTGAAGATGGAGAAACAAGTGATCAACAATTGAACCAAAGTATGGACACAGGCT $\begin{array}{llllllllllllllllllllllllllll}Y & I & I & E & D & G & E & T & S & D & Q & Q & L & N & Q & S & M & D & T & G\end{array}$ CTCCAGCAGAACTGTCTCCTACTACTCTCTCCCCTGTTAATCATAGCCTGGATTTGCAGC $\begin{array}{llllllllllllllllllll}S & P & A & E & L & S & P & T & T & L & S & P & V & N & H & S & L & D & L & Q\end{array}$ CAGTTACTTACTCAGAACCTGCGTTTTGgTGTTCAATAGCATATTATGAATGAAACCAGA $\begin{array}{lllllllllllllllll}\mathrm{P} & \mathrm{V} & \mathrm{T} & \mathrm{Y} & \mathrm{S} & \mathrm{E} & \mathrm{P} & \mathrm{A} & \mathrm{F} & \mathrm{W} & \mathrm{C} & \mathrm{S} & \mathrm{I} & \mathrm{A} & \mathrm{Y} & \mathrm{Y} & \mathrm{E}\end{array}$

\section{FIG. 5.10. Partial cDNA sequence of $\operatorname{Smad} 2$}

Partial nucleotide sequence of Smad 2 amplified from skeletal muscle of $I$. tridecemlineatus and translated amino acid sequence. The ground squirrel gene sequence closely matches the comparable open reading frames of human, mouse and rat Smad 2 genes. 
(A)
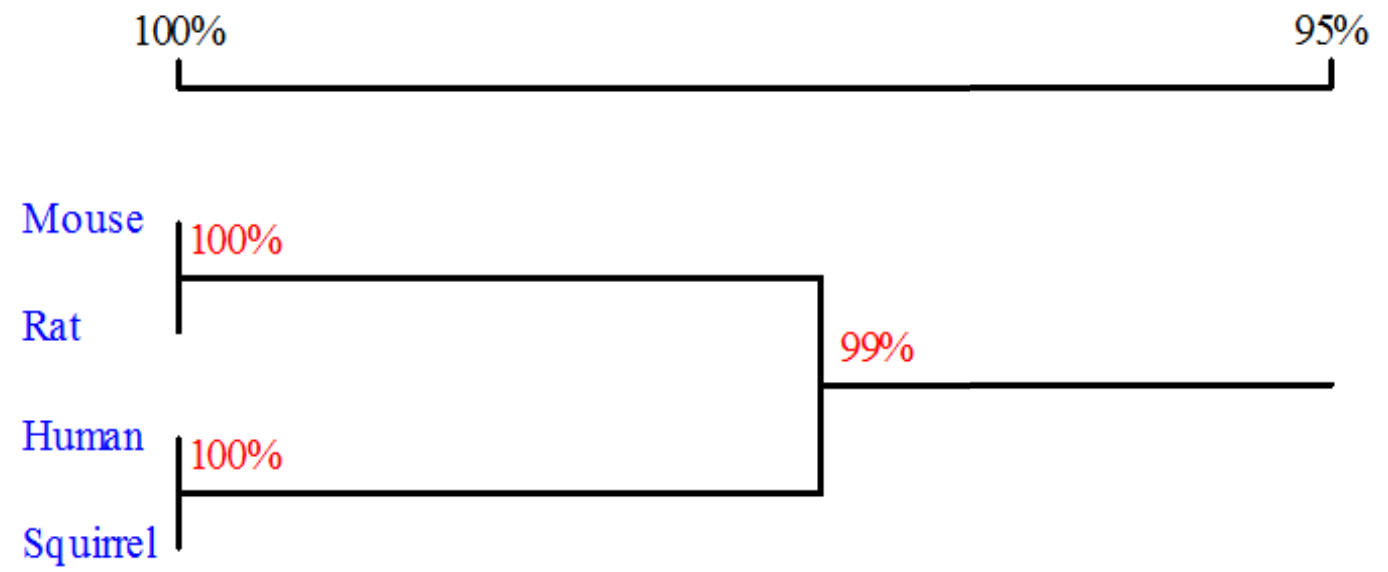

(B)

$\begin{array}{llll} & \text { Mouse } & \text { Rat Human } & \text { squiret } \\ \text { Mouse } & 100 \% & & \\ \text { Rat } & 99.8 \% 100 \% & \\ \text { Human } & 99.6 \% 99.4 \% 100 \% \\ \text { Squirrel } & 99.4 \% 99.1 \% 100.0 \% 100 \%\end{array}$

\section{FIG 5.11. Smad 2 homology alignment.}

(A) Homology tree produced from alignment of I. tridecemlineatus Smad 2 protein sequence with human (Homo sapiens), rat (Rattus norvegicus), and mouse (Mus musculus) sequences. The percentage values correspond to degree of homology between species. (B) Homology matrix representing degree of conservation of I. tridecemlineatus Smad 2 sequence compared with Smad 2 from other mammals. 

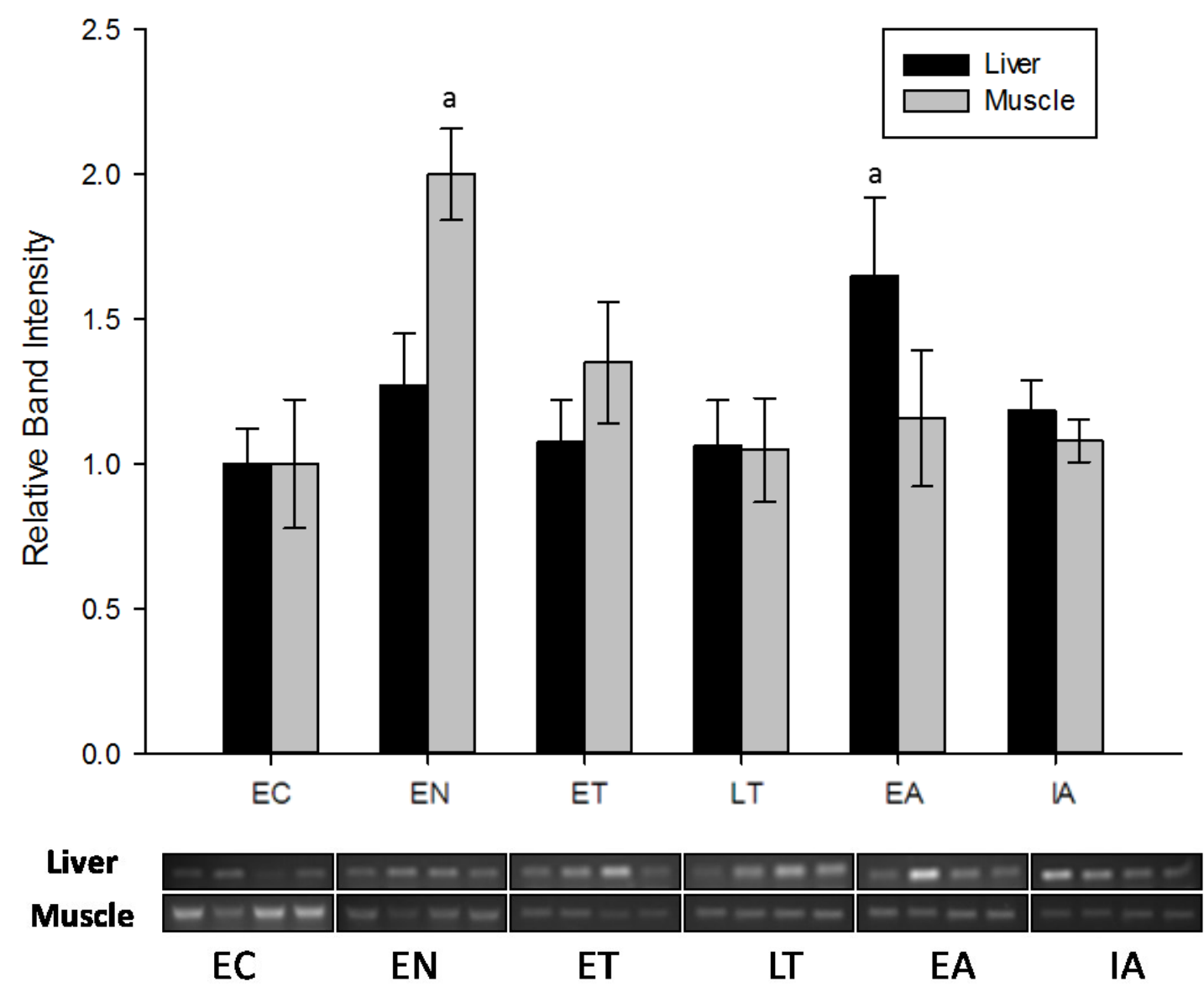

FIG. 5.12. Transcriptional regulation of $S \mathrm{mad} 3$ in liver and skeletal muscle of $I$. tridecemlineatus as determined by RT-PCR.

Smad3 mRNA transcript levels in liver and muscle of 13-lined ground squirrels over the stages of the torpor-arousal cycle. SyBR green stained agarose gels are shown for Smad3. Data are means \pm S.E.M., $\mathrm{n}=4$ independent trials on tissues from different animals. a-Significantly different from corresponding euthermic value $(\mathrm{P}<0.05$, ANOVA with post hoc Holm-Sidak test). 
CCCCACAACTAAGCCGTCGTATGCAGCAGAGACTTCTGCTTATGAGGCCATCACCACGCA $\begin{array}{llllllllllllllllllll}\mathrm{P} & \mathrm{T} & \mathrm{T} & \mathrm{K} & \mathrm{P} & \mathrm{S} & \mathrm{Y} & \mathrm{A} & \mathrm{A} & \mathrm{E} & \mathrm{T} & \mathrm{S} & \mathrm{A} & \mathrm{Y} & \mathrm{E} & \mathrm{A} & \mathrm{I} & \mathrm{T} & \mathrm{T} & \mathrm{Q}\end{array}$ GAACGTCAACACCAAGTGCATCACCATCCCCAGGTCTTTGGATGGCCGGCTGCAGGTGTC $\begin{array}{lllllllllllllllllllll}\mathrm{N} & \mathrm{V} & \mathrm{N} & \mathrm{T} & \mathrm{K} & \mathrm{C} & \mathrm{I} & \mathrm{T} & \mathrm{I} & \mathrm{P} & \mathrm{R} & \mathrm{S} & \mathrm{L} & \mathrm{D} & \mathrm{G} & \mathrm{R} & \mathrm{L} & \mathrm{Q} & \mathrm{V} & \mathrm{S}\end{array}$ CCATCGGAAGGGGCTCCCCCATGTCATCTACTGCCGCCTGTGGCGATGGCCTGACCTGCA $\begin{array}{llllllllllllllllllll}\mathrm{H} & \mathrm{R} & \mathrm{K} & \mathrm{G} & \mathrm{L} & \mathrm{P} & \mathrm{H} & \mathrm{V} & \mathrm{I} & \mathrm{Y} & \mathrm{C} & \mathrm{R} & \mathrm{L} & \mathrm{W} & \mathrm{R} & \mathrm{W} & \mathrm{P} & \mathrm{D} & \mathrm{L} & \mathrm{H}\end{array}$ CAGCCACCATGAGCTGCGGgCCATGGAGCTGTGTGAATTCGCCTTCAACATGAAGAAGgA $\begin{array}{llllllllllllllllllll}\mathrm{S} & \mathrm{H} & \mathrm{H} & \mathrm{E} & \mathrm{L} & \mathrm{R} & \mathrm{A} & \mathrm{M} & \mathrm{E} & \mathrm{L} & \mathrm{C} & \mathrm{E} & \mathrm{F} & \mathrm{A} & \mathrm{F} & \mathrm{N} & \mathrm{M} & \mathrm{K} & \mathrm{K} & \mathrm{D}\end{array}$ CGAGGTCTGCGTGAACCCCTACCACTACCAGAGGGTAGAGACACCAGTTCTACCTCCTGT $\begin{array}{lllllllllllllllllllllllll}E & \mathrm{~V} & \mathrm{C} & \mathrm{V} & \mathrm{N} & \mathrm{P} & \mathrm{Y} & \mathrm{H} & \mathrm{Y} & \mathrm{Q} & \mathrm{R} & \mathrm{V} & \mathrm{E} & \mathrm{T} & \mathrm{P} & \mathrm{V} & \mathrm{L} & \mathrm{P} & \mathrm{P} & \mathrm{V}\end{array}$ GCTGGTGCCACGGCACACAGAGATCCCGGCCGAGTTCCCCCCGCTGGACGACTACAGCCA

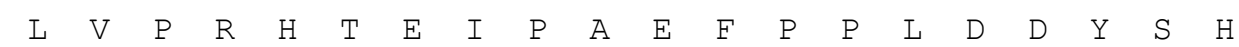
TTCCATCCCTGAGAACACCAACTTCCCCGCTGGCATCGAGCCCCAGAGCAATATTCCAGA $\begin{array}{llllllllllllllllllllllll}\mathrm{S} & \mathrm{I} & \mathrm{P} & \mathrm{E} & \mathrm{N} & \mathrm{T} & \mathrm{N} & \mathrm{F} & \mathrm{P} & \mathrm{A} & \mathrm{G} & \mathrm{I} & \mathrm{E} & \mathrm{P} & \mathrm{Q} & \mathrm{S} & \mathrm{N} & \mathrm{I} & \mathrm{P} & \mathrm{E}\end{array}$ GACCCCACCCCCTGGCTACCTGAGTGAAGATGGAGAGACCAGCGACCACCAGATGAACCA $\begin{array}{llllllllllllllllllllllll}T & P & P & P & G & Y & L & S & E & D & G & E & T & S & D & H & Q & M & N & H\end{array}$ CAGCATGGACGCAGGTTCTCCAAATCTGTCCCCAAATCCGATGTCCCCAGCACACAATAA $\begin{array}{lllllllllllllllllllllll}\mathrm{S} & \mathrm{M} & \mathrm{D} & \mathrm{A} & \mathrm{G} & \mathrm{S} & \mathrm{P} & \mathrm{N} & \mathrm{L} & \mathrm{S} & \mathrm{P} & \mathrm{N} & \mathrm{P} & \mathrm{M} & \mathrm{S} & \mathrm{P} & \mathrm{A} & \mathrm{H} & \mathrm{N} & \mathrm{N}\end{array}$ CCTGGACCTGCAGCCAGTCACCTACTGCGAGCCAGCCTTCTGGTGCTCCATCTCCTACTA $\begin{array}{lllllllllllllllllllllll}\mathrm{L} & \mathrm{D} & \mathrm{L} & \mathrm{Q} & \mathrm{P} & \mathrm{V} & \mathrm{T} & \mathrm{Y} & \mathrm{C} & \mathrm{E} & \mathrm{P} & \mathrm{A} & \mathrm{F} & \mathrm{W} & \mathrm{C} & \mathrm{S} & \mathrm{I} & \mathrm{S} & \mathrm{Y} & \mathrm{Y}\end{array}$ CGAGCTGAACCAACCAAAC

$\begin{array}{llllllllll} & E & L & N & Q & P & N\end{array}$

\section{FIG. 5.13. Partial cDNA sequence of Smad 3}

Partial nucleotide sequence of Smad 3 amplified from muscle of $I$. tridecemlineatus along with translated amino acid sequence. The nucleotide sequence closely matches the corresponding open reading frames of human, mouse and rat Smad 3. 
(A)

$100 \%$

I

\begin{tabular}{l|l} 
Mouse & \\
Rat & $100 \%$ \\
Human & $100 \%$ \\
Squirrel &
\end{tabular}

(B)

\begin{tabular}{|c|c|c|c|c|}
\hline & Mouse & Rat & Human & squirrel \\
\hline Mouse & $100 \%$ & & & \\
\hline Rat & $99.8 \%$ & $100 \%$ & & \\
\hline Human & $99.8 \%$ & $100.0 \%$ & $100 \%$ & \\
\hline Squirrel & $99.8 \%$ & $100.0 \%$ & $100.0 \%$ & $100 \%$ \\
\hline
\end{tabular}

\section{FIG 5.14. Smad 3 homology alignment}

(A) Homology tree produced from alignment of I. tridecemlineatus Smad 3 protein sequence with human (Homo sapiens), rat (Rattus norvegicus), and mouse (Mus musculus). The percentage values correspond to degree of homology between species. (B) Homology matrix representing degree of conservation of I. tridecemlineatus Smad 3 sequence compared with Smad 3 from other mammals. 

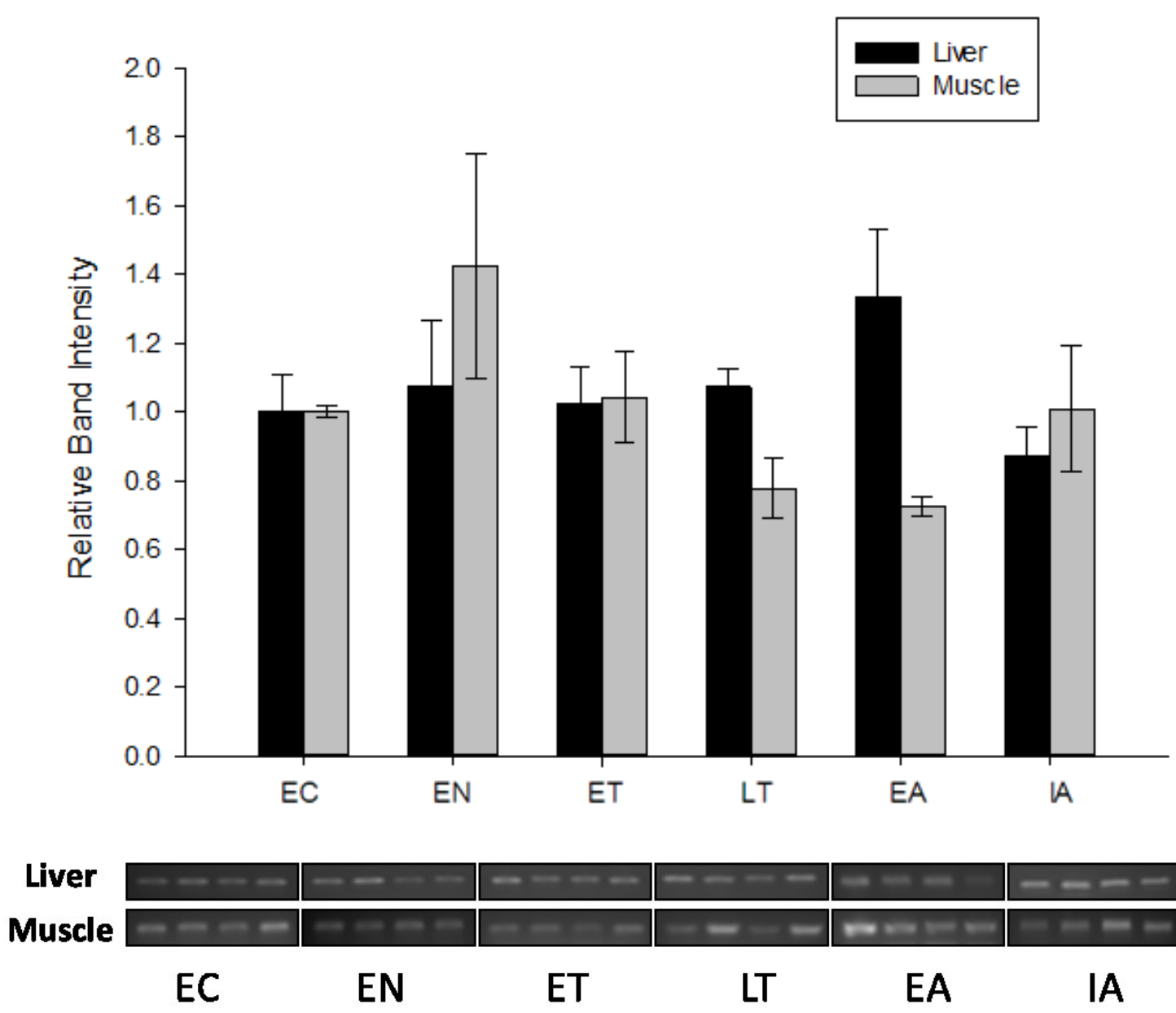

FIG. 5.15. Transcriptional regulation of $S m a d 4$ in liver and skeletal muscle of $I$. tridecemlineatus as determined by RT-PCR.

Smad4 mRNA transcript levels in the liver and muscle of 13-lined ground squirrels over the stages of the torpor-arousal cycle. SyBR green stained agarose gels are shown for Smad4. Data are means \pm S.E.M., $\mathrm{n}=4$ independent trials on tissues from different animals. 
$\begin{array}{llllllllllllllllllllllllllll}K & W & S & S & S & S & * & M & C & Y & N & T & E & I & I & G & W & K & T\end{array}$

CAGCTCCACCAAGTATGTTGGTGAAGGATGAATATGTTCATGACTTTGAGGGACAGCCAT

$\begin{array}{llllllllllllllllllll}S & A & P & P & S & M & L & V & K & D & E & Y & V & H & D & F & E & G & Q & P\end{array}$ CATTATCCACAGAAGGACATTCAATTCAAACCATCCAGCATCCACCAAGTAATCGTGCTT

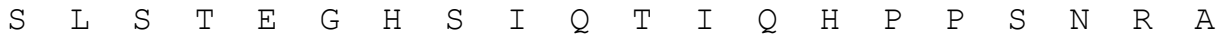
CGACGGAGACATACAGTACCCCAGCTCTTTTAGCCCCATCTGAGTCAAATGCTACCAGCA $\begin{array}{llllllllllllllllllll}S & T & E & T & Y & S & T & P & A & L & L & A & P & S & E & S & N & A & T & S\end{array}$ CCACCAACTTTCCCAACATTCCTGTGGCTTCCACAAGTCAGCCTGCCAGTATTCTGGCAG $\begin{array}{llllllllllllllllllll}T & \mathrm{~T} & \mathrm{~N} & \mathrm{~F} & \mathrm{P} & \mathrm{N} & \mathrm{I} & \mathrm{P} & \mathrm{V} & \mathrm{A} & \mathrm{S} & \mathrm{T} & \mathrm{S} & \mathrm{Q} & \mathrm{P} & \mathrm{A} & \mathrm{S} & \mathrm{I} & \mathrm{L} & \mathrm{A}\end{array}$ GTAGTCATAGTGAAGGACTGTTGCAAATAGCTTCAGGGCCTCAGCCAGGACAGCAGCAGA

FIG. 5.16. Partial cDNA sequence of Smad 4

Partial nucleotide sequence of Smad 4 amplified from muscle of $I$.

tridecemlineatus and translated amino acid sequence. The ground squirrel gene sequence closely matches the comparable open reading frames of human, mouse and rat Smad 4 genes. 
(A)

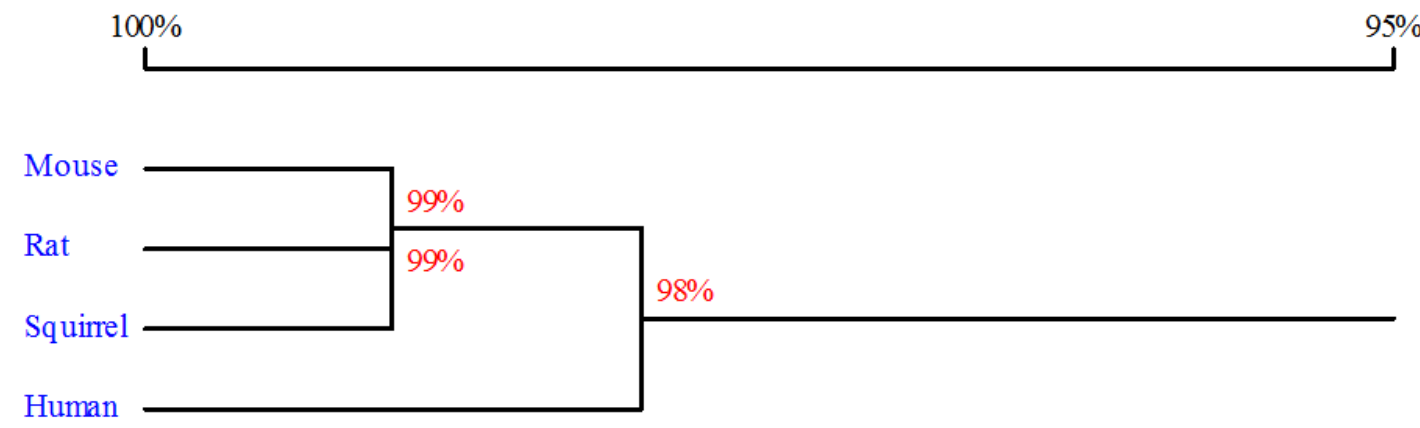

(B)

\begin{tabular}{|c|c|c|c|c|}
\hline & Mouse & Rat & Human & squistel \\
\hline Mouse & 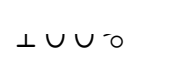 & & & \\
\hline Rat & $98.5 \%$ & $100 \%$ & & \\
\hline Human & $97.0 \%$ & $98.4 \%$ & $100 \%$ & \\
\hline Squirrel & $99.0 \%$ & $99.4 \%$ & $99.0 \%$ & $100 \%$ \\
\hline
\end{tabular}

\section{FIG 5.17. Smad 3 homology alignment.}

(A) Homology tree produced from alignment of I. tridecemlineatus Smad 3 protein sequence with human (Homo sapiens), rat (Rattus norvegicus), and mouse (Mus musculus). The percentage values correspond to degree of homology between species. (B) Homology matrix representing degree of conservation of I. tridecemlineatus Smad 4 sequence compared with Smad 4 from other mammals. 


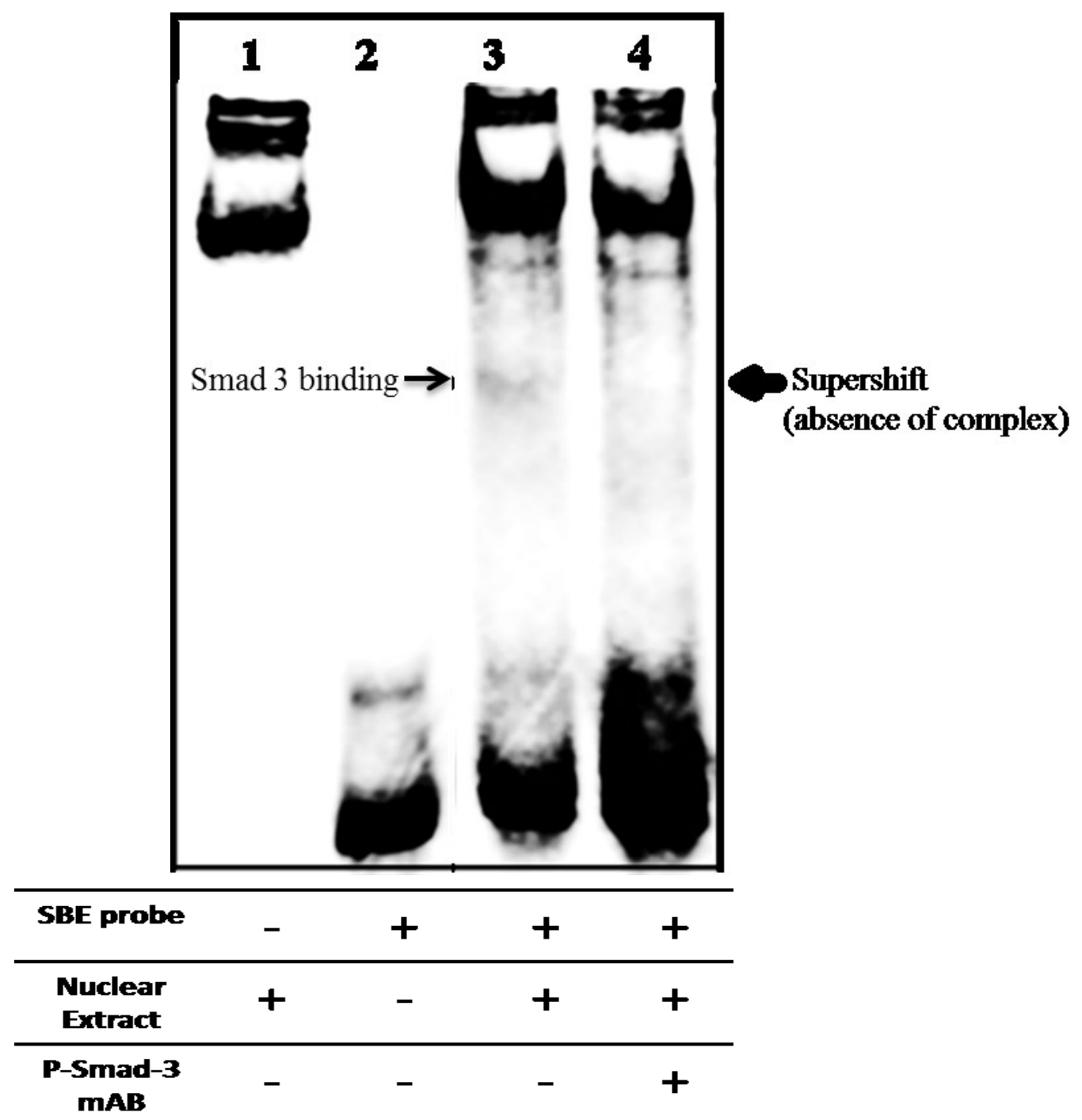

FIG. 5.18 Smad3 DNA binding complex in I. tridecemlineatus liver as determined by electrophoretic mobility shift assay (EMSA) on PAGE

Binding of Smad 3 transcription factor complex to double stranded DNA probes containing the Smad Binding Element sequence in nuclear extracts of I. tridecemlineatus liver. Smad 3 protein binding is confirmed with a supershift of the complex when binding the monoclonal phospho-Smad3 antibody. Lane 1 is a negative control with no probe, lane 2 is a negative control with no nuclear extract, lane 3 contains nuclear extract and probe, and lane 4 contains the same components as lane 3 but with the addition of pSmad 3 antibody. 


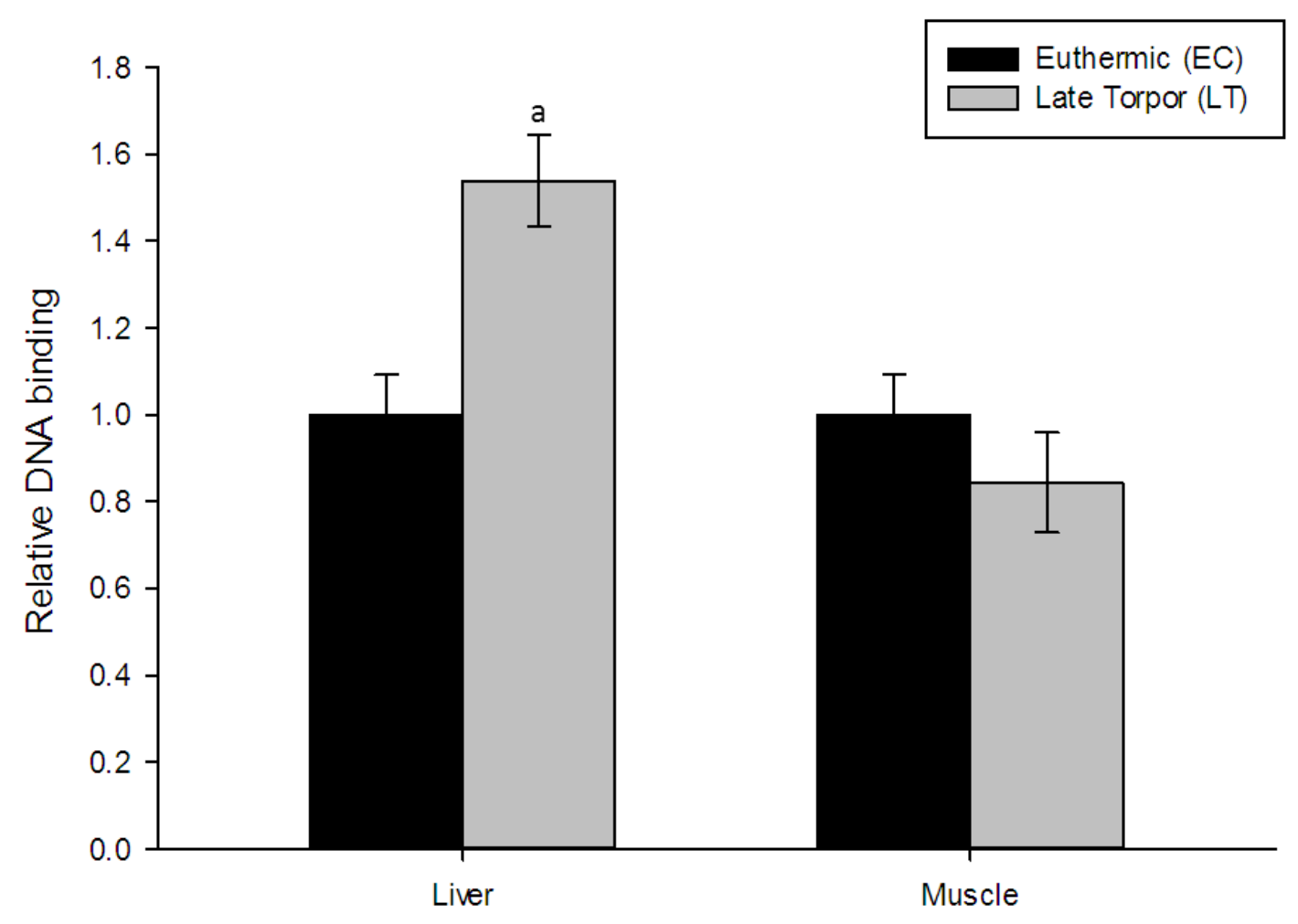

FIG. 5.19 DNA binding activity of Smad 3 transcription factor in liver and skeletal muscle of I. tridecemlineatus as determined by enzyme-linked immunosorbent assay (ELISA).

Relative DNA-binding activity of Smad 3 protein comparing euthermic controls and late torpor states. Data are means \pm S.E.M., $n=4$ independent trials on tissues from different animals. a-Significantly different from corresponding euthermic values $(\mathrm{P}<0.05$, Student's t-test $)$. 


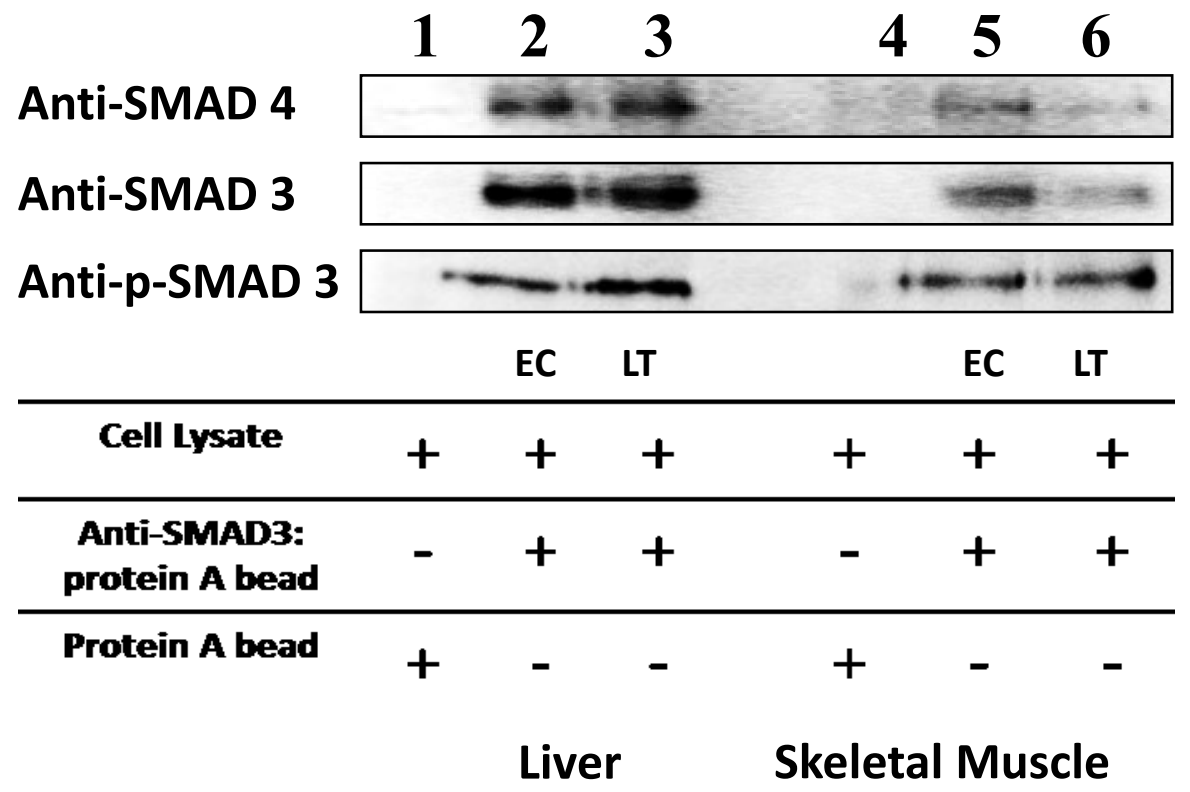

FIG. 5.20. Transcriptional complex of Smad 3 and Smad 4 proteins in liver and skeletal muscle of I. tridecemlineatus as determined by co-immunoprecipitation.

Samples were co-immunoprecipitated with anti-Smad 3 antibody immobilized and crosslinked to protein-A agarose beads. Samples were separated on 10\% SDS-PAGE and assessed for the presence of Smad 3, phosphorylated Smad 3, and Smad 4 via Western blotting. Lanes 1 and 4 have no crosslinked antibody: protein A beads, lanes 2 and 3 are liver samples EC and LT respectively, and lanes 5 and 6 are skeletal muscle samples EC and LT respectively. 


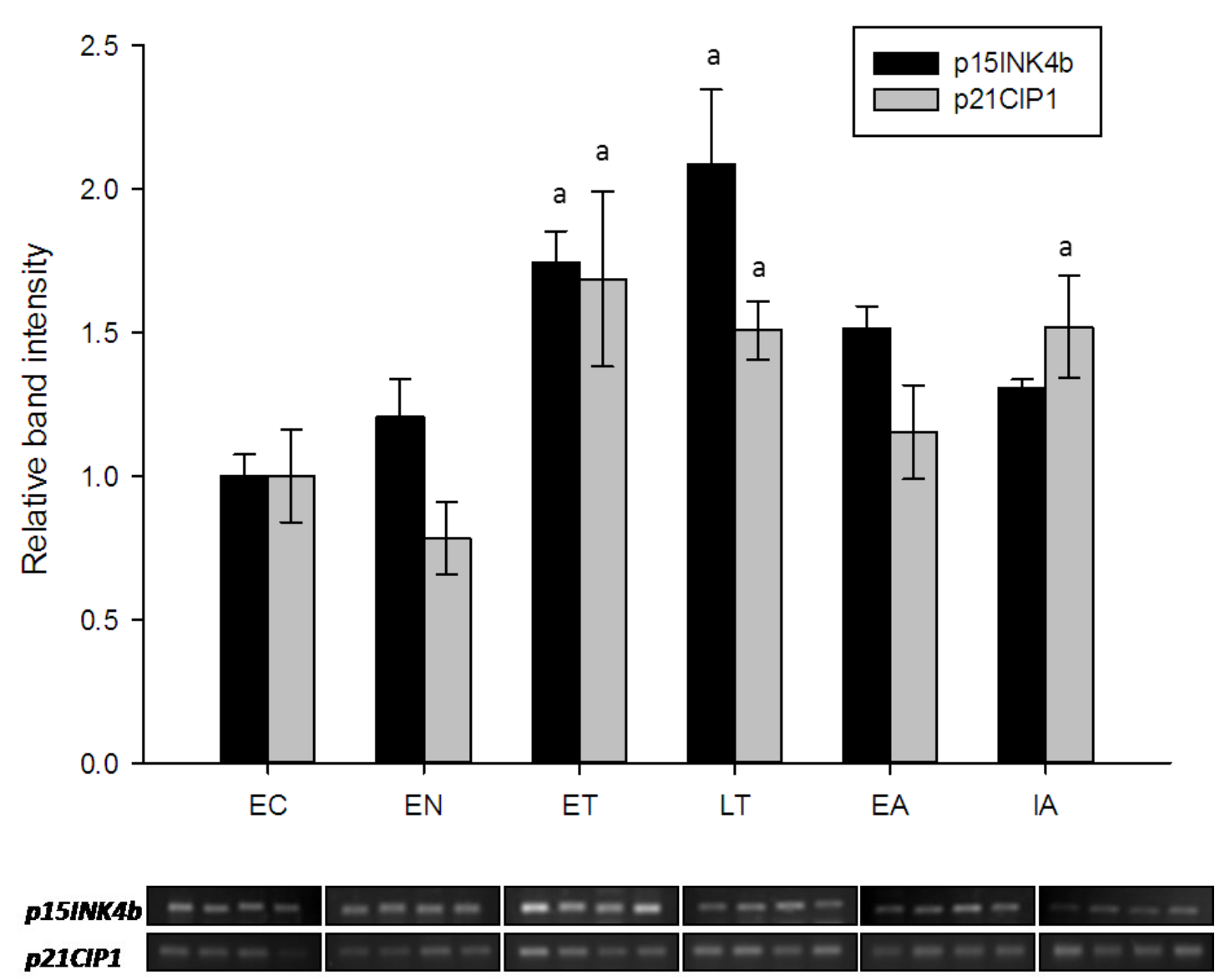

FIG. 5.21. Transcriptional regulation of cell cycle inhibitors in liver of $I$. tridecemlineatus as determined by RT-PCR.

Effects of hibernation on $p 15 I N K 4 b$ and $p 21 C I P 1$ mRNA transcript levels in liver of 13-lined ground squirrels over the stages of the torpor-arousal cycle. PCR products were visualized on SyBR green stained agarose gels. Data are means \pm S.E.M., $n=4$ independent trials on tissues from different animals. a-Significantly different from corresponding euthermic values ( $\mathrm{P}<0.05$, ANOVA with post hoc Holm-Sidak test). 


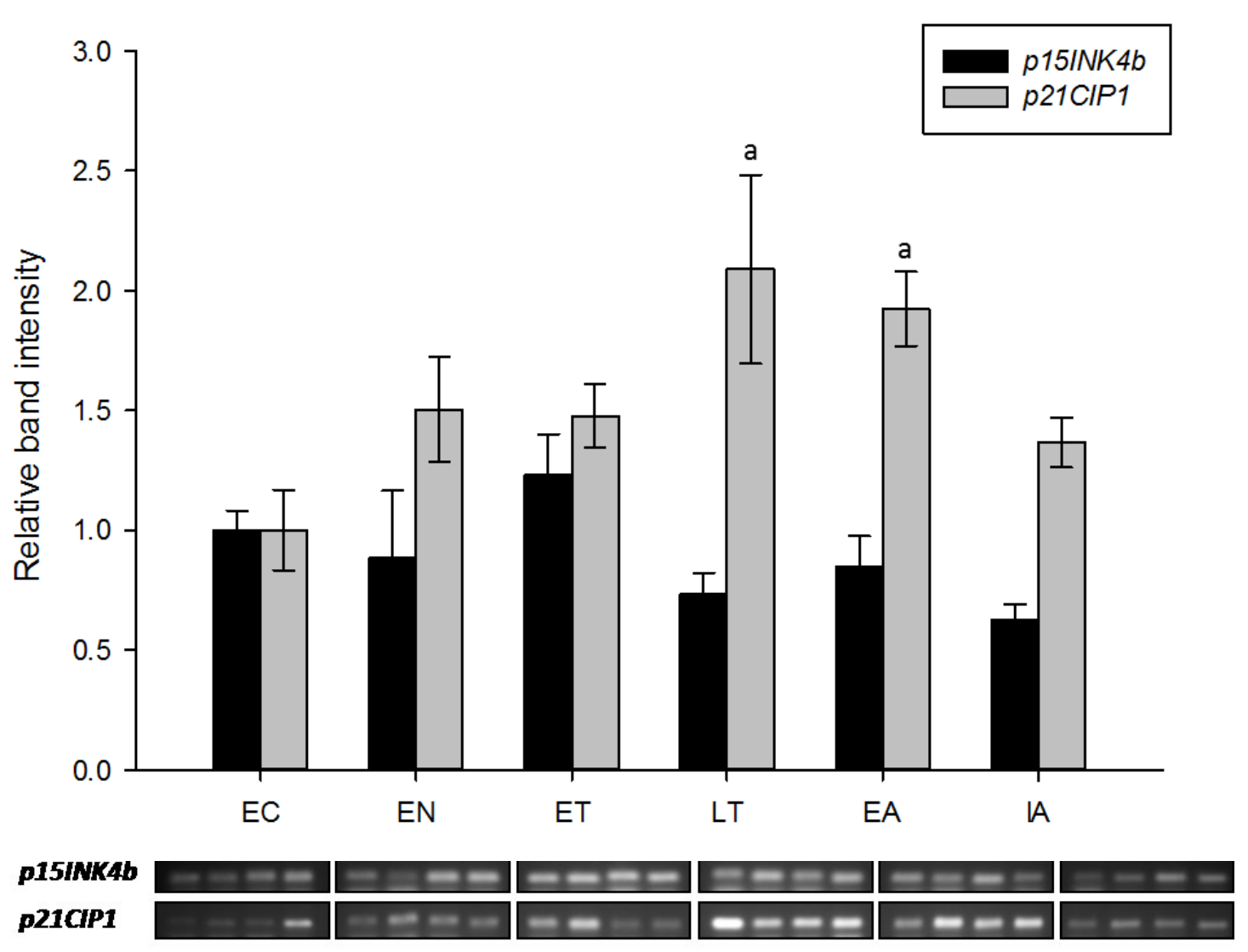

FIG. 5.22. Transcriptional regulation of cell cycle inhibitors in skeletal muscle of $I$. tridecemlineatus as determined by RT-PCR.

Effects of hibernation on $p 15 I N K 4 b$ and $p 21 C I P 1$ mRNA transcript levels in the skeletal muscle of 13-lined ground squirrels over the stages of the torpor-arousal cycle. SyBR green stained agarose gels are shown for $p 15 I N K 4 b$ and $p 21 C I P 1$ genes. Data are means \pm S.E.M., $\mathrm{n}=4$ independent trials on tissues from different animals. a-Significantly different from corresponding euthermic values $(\mathrm{P}<0.05$, ANOVA post hoc Holm-Sidak test). 


\section{FIG. 5.23. Partial cDNA sequence of $p 15 I N K 4 b$}

Partial nucleotide sequence of $p 15 I N K 4 b$ amplified from muscle of $I$. tridecemlineatus and translated amino acid sequence. The ground squirrel gene sequence closely matches the comparable open reading frames of human, mouse and rat genes. 
(A)
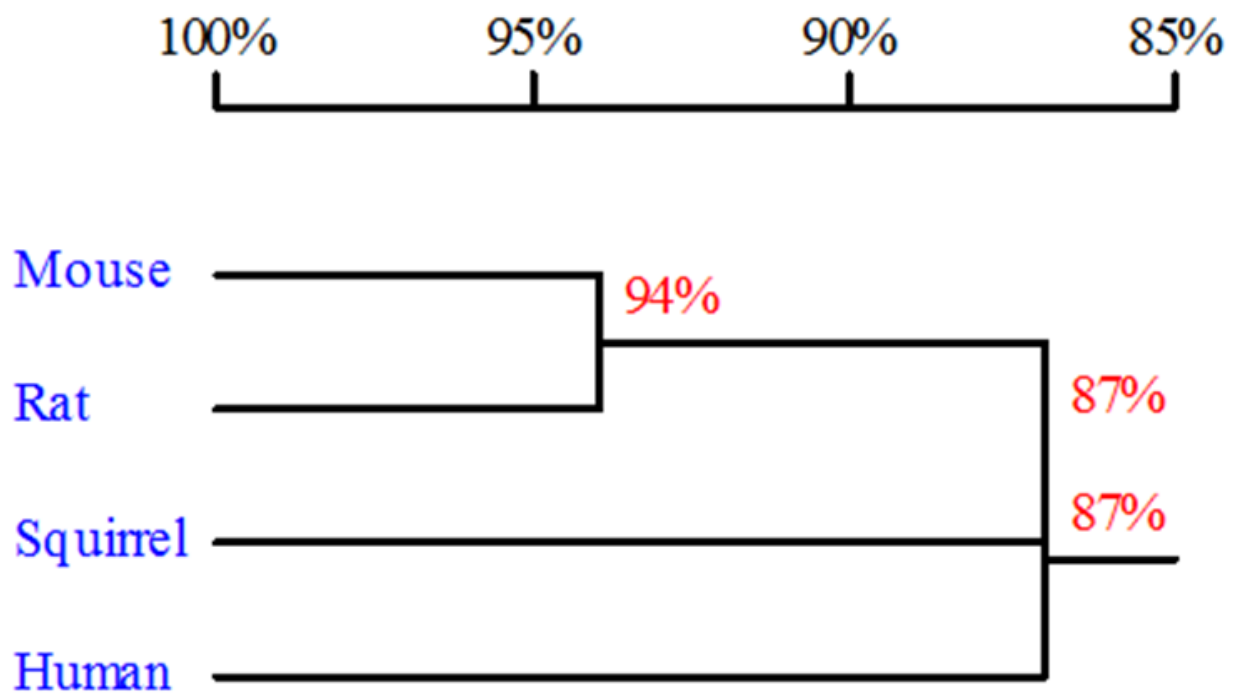

(B)

$\begin{array}{lll} & \text { Mouse Rat Human squrrel } \\ \text { Mouse } & & \\ \text { Rat } & \text { u } & \\ \text { Human } & 94.4 \% 100 \% \\ \text { Squirrel } & 86.9 \% 86.3 \% 100 \% \\ & 88.5 \% 86.3 \% 87.0 \% 100 \%\end{array}$

\section{FIG 5.24. p15INK4b homology alignment.}

(A) Homology tree produced from alignment of I. tridecemlineatus p15INK4b protein sequence with human (Homo sapiens), rat (Rattus norvegicus), and mouse (Mus musculus). The percentage values correspond to the degree of homology between species. (B) Homology matrix representing degree of conservation of I. tridecemlineatus p15INK4b sequence compared with p15INK4b from other mammals. 


\section{FIG. 5.25. Partial cDNA sequence of p21CIP1}

Partial nucleotide sequence of p21CIP1 amplified from muscle of $I$.

tridecemlineatus and translated amino acid sequence. The ground squirrel gene sequence closely matches the corresponding open reading frames of the human, mouse and rat genes. 
(A)
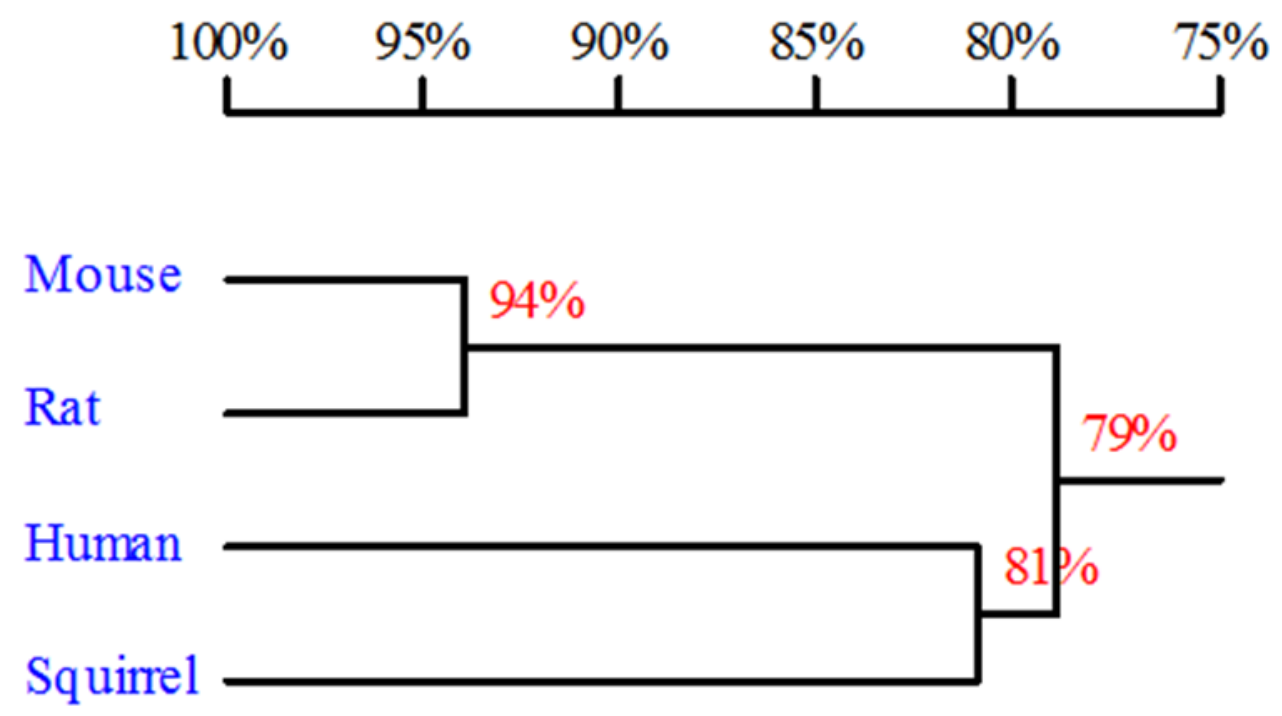

(B)

$$
\text { Mouse rat Human squirrel }
$$

Mouse

Rat

Human

Squirrel $\perp U \cup \div$

$93.7 \% 100 \%$

$79.2 \% 76.5 \% 100 \%$

$82.2 \% 78.1 \% 80.9 \% 100 \%$

FIG 5.26. p21CIP1 homology alignment.

(A) Homology tree produced from alignment of I. tridecemlineatus p21CIP1 protein sequence with human (Homo sapiens), rat (Rattus norvegicus), and mouse (Mus musculus). The percentage values correspond to degree of homology between species.

(B) Homology matrix representing degree of conservation of I. tridecemlineatus p21CIP1 sequence compared with p21CIP1 from other mammals. 


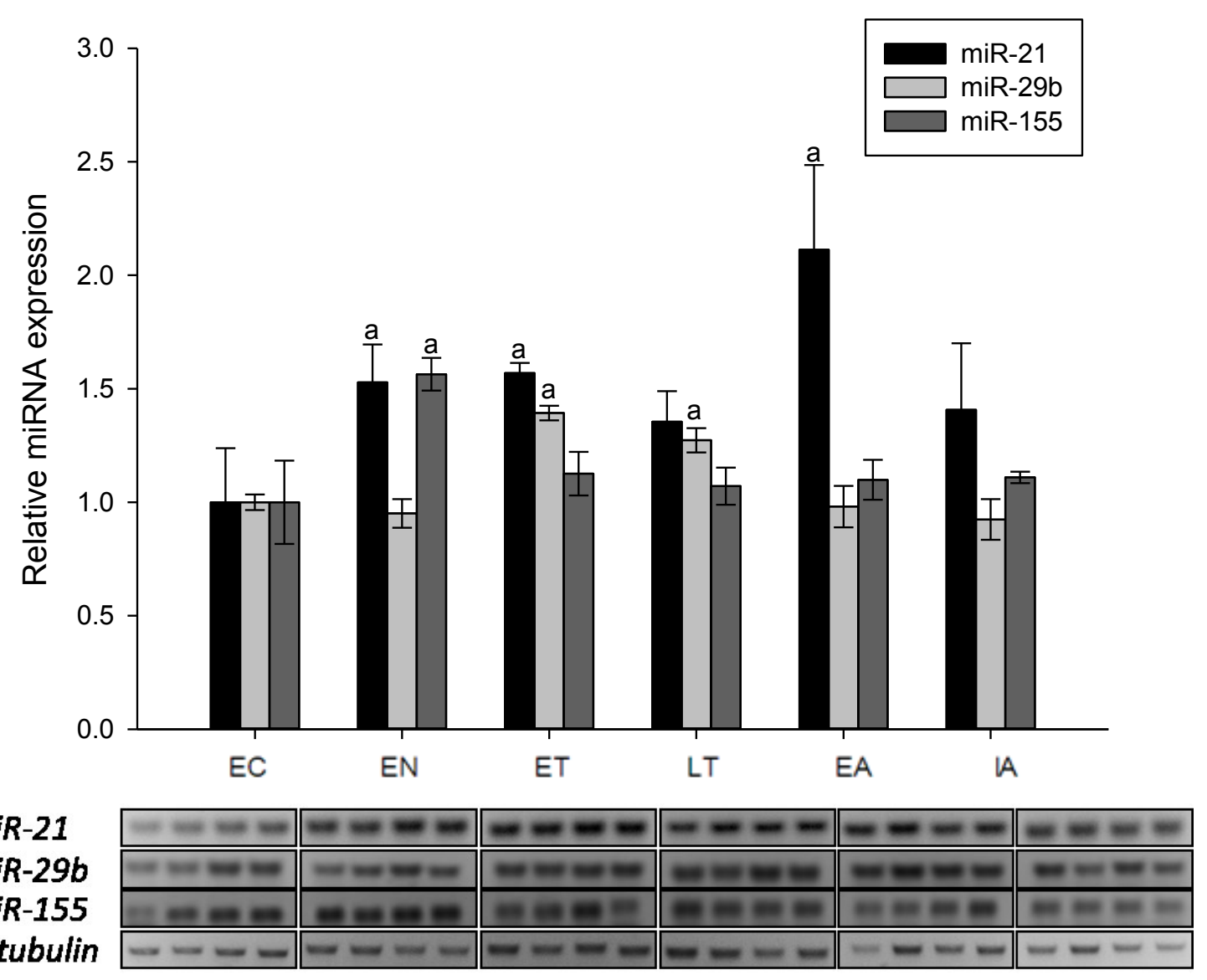

FIG. 5.27. Transcriptional regulation of Smad downstream microRNA targets in liver of I. tridecemlineatus as determined by RT-PCR.

Effects of hibernation on microRNA $m i R-21, m i R-29 b$, and $m i R-155$ transcript levels in the liver of 13-lined ground squirrels over the stages of the torpor-arousal cycle. SyBR green stained agarose gels are shown for $m i R-21, m i R-29 b$, and $m i R-155$ transcripts and for the housekeeping gene, $\alpha$-tubulin. Data are means \pm S.E.M., $\mathrm{n}=4$ independent trials on tissues from different animals. a-Significantly different from corresponding euthermic values $(\mathrm{P}<0.05$, ANOVA post hoc Holm-Sidak test). 

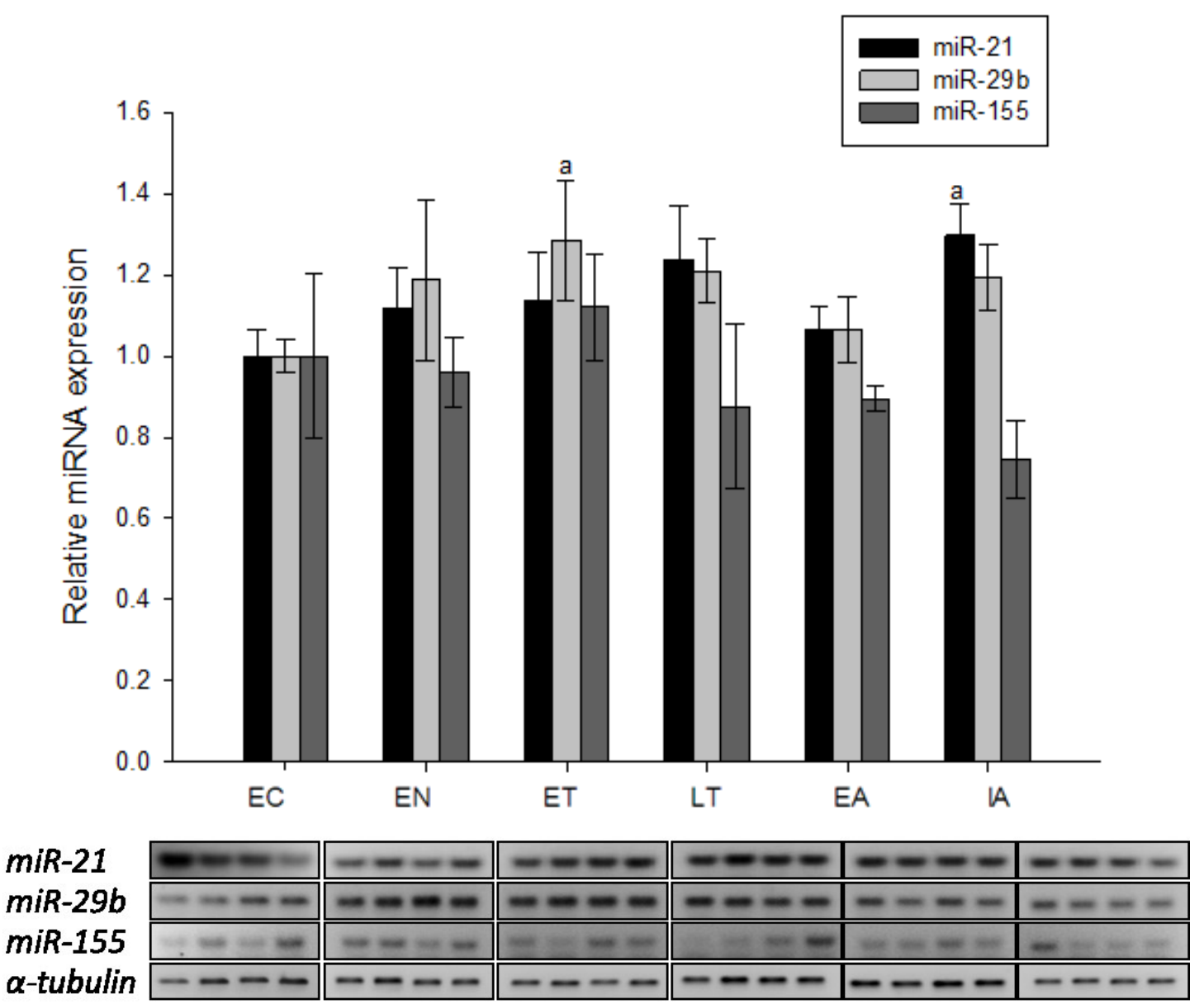

FIG. 5.28. Transcriptional regulation of Smad downstream microRNA targets in skeletal muscle of I. tridecemlineatus as determined by RT-PCR.

Effects of hibernation on microRNA $m i R-21, m i R-29 b$, and $m i R-155$ transcript levels in the skeletal muscle of 13-lined ground squirrels over the stages of the torporarousal cycle. SyBR green stained agarose gels are shown for $m i R-21, m i R-29 b$, and $m i R-$ 155 transcripts and for the housekeeping gene, $\alpha$-tubulin. Data are means \pm S.E.M., $\mathrm{n}=4$ independent trials on tissues from different animals. a-Significantly different from corresponding euthermic values $(\mathrm{P}<0.05$, ANOVA post hoc Holm-Sidak test). 


\title{
CHAPTER 6
}

\section{REGULATION OF CELL CYCLE PROGRESSION IN HIBERNATING GROUND SQUIRRELS}

\author{
Published in Cell Cycle
}

Reprint with permission from Landes Bioscience 


\subsection{INTRODUCTION}

The progression of life in all organisms involves a fundamental growth and proliferation process, by which physical mass is increased. A conserved biological process in both unicellular and multicellular organism that regulates cellular growth is the cell cycle. The eukaryotic cell cycle is comprised four distinct phases $\left(G_{1}, S, G_{2}\right.$, and $M$ phases) that control and regulate the progression of cell division (FIG. 6.1). When cell cycle progression is first initiated, dividing cells exit the $G_{0}$ state to enter $G_{1}$, the first gap phase of the cell cycle, that functions to ensure that conditions in the cellular environment are suitable for DNA replication. Cells then progress into the synthesis phase (S) where DNA replication takes place and this is then followed by the second gap phase $\left(\mathrm{G}_{2}\right)$ that functions to ensure that proper DNA replication has occurred before entry into mitosis. Through mitosis $(\mathrm{M})$, the replicated chromosomes are segregated into two identical daughter cells (Schafer, 1998; Biggar and Storey, 2009). The progression through the phases of the cell cycle is tightly regulated by two classes of proteins: cyclins and cyclin dependent kinases (Cdks) (Schafer, 1998). The binding of the regulatory cyclin proteins to Cdks is essential in the formation of the kinase complex, which upon dephosphorylation by Cdc25 phosphatases, fully activates the kinase activity of the cyclin/Cdk complex (Bloom and Cross, 2007; Ohtani and Nevins, 1994). The activated Cdk then functions to phosphorylate a number of different substrates, catalyzing the progression of cell division (Schafer, 1998). The complexity of eukaryotic cell cycle regulation derives from the multiple cyclins that interact with multiple Cdks, with each distinct combination acting to regulate a specific phase of the cell cycle (Bloom and 
Cross, 2007).

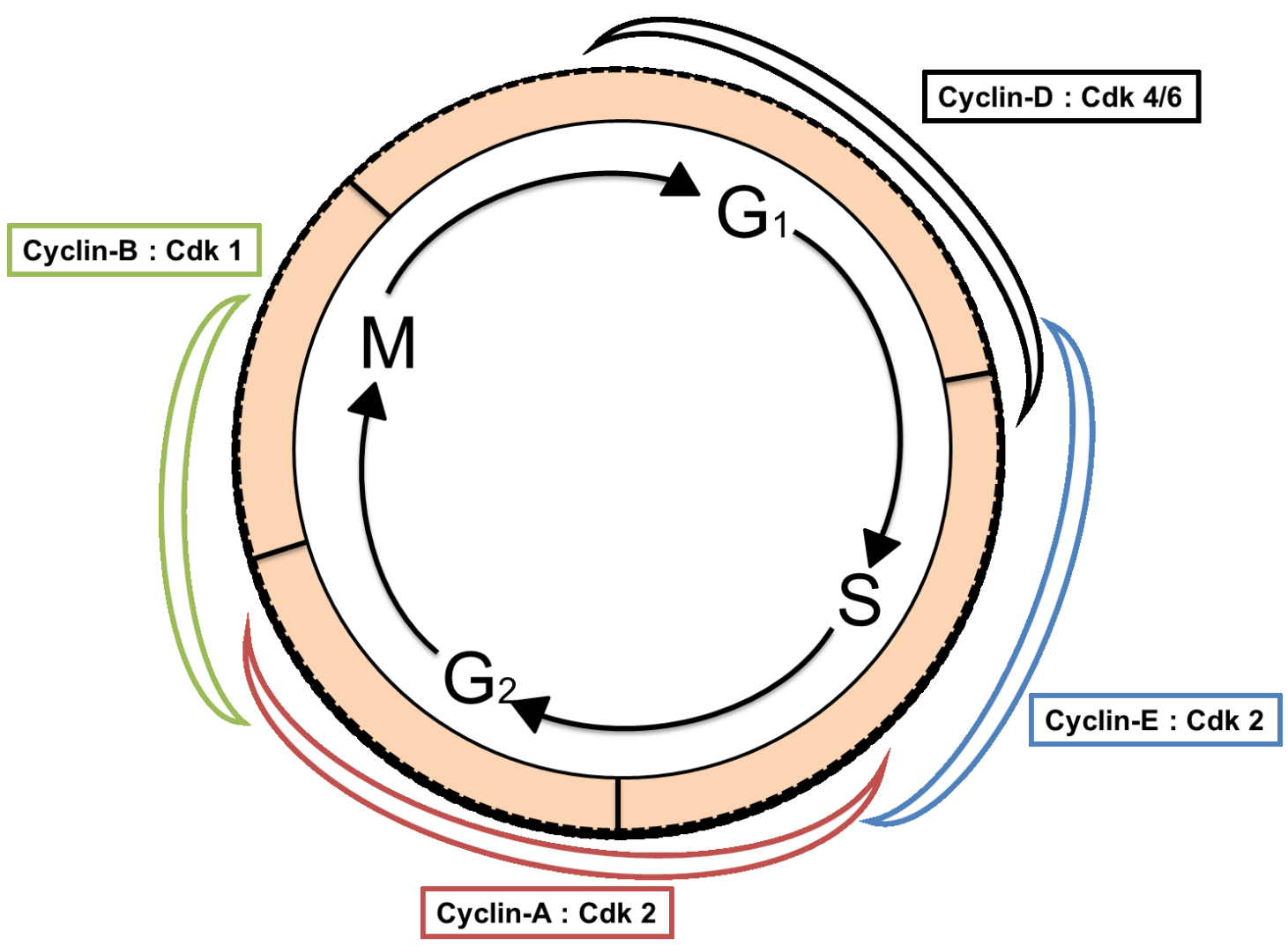

FIG. 6.1. Stages of the cell cycle. Each of the four stages in the cell cycle is regulated by a combination of different cyclins and Cdk proteins. The kinase activity of the cyclin:Cdk complex activates substrates that regulate the progression and completion of each phase. 
In the $\mathrm{G}_{1}$ phase, cyclin $\mathrm{D}$ along with $\mathrm{Cdk} 4 / 6$ phosphorylate the retinoblastoma tumour suppressor gene $(\mathrm{Rb})$, disrupting the inhibitory effect of $\mathrm{Rb}$ on the transcription factor E2F-1. Activated E2F-1 then promotes the transcription of several genes including cyclin $E$, cyclin $A$, and $C d c 25$ whose protein products are involved in later stages of the cell cycle (Ohtani and Nevins, 1994; Johnson and Walker, 1999). As the cell approaches the $\mathrm{G}_{1} \rightarrow \mathrm{S}$ transition phase, the cyclin $\mathrm{E} / \mathrm{Cdk} 2$ complex functions to regulate entry into the $\mathrm{S}$ phase by maintaining $\mathrm{Rb}$ hyperphosphorylation, as well as phosphorylation of histone H1 (Hinds et al., 1992; Contreras et al., 2003). While in S phase, cyclin A complexes with Cdk 2 to regulate the early stages of S phase, followed by a complex with Cdk 1 in late S phase as well as during the $\mathrm{G}_{2} \rightarrow \mathrm{M}$ transition phase (Walker and Maller, 1991; Katsuno et al., 2009). Once the cell enters mitosis, the progression towards cell division is regulated by cyclin B through its complex with Cdk1 (Jackman, 2003). Although cyclins and Cdks play a major role in the positive regulation of cell cycle progression, two families of Cdk inhibitors (CKI) negatively regulate the cell cycle: $\mathrm{Cdk}$ inhibitors (INK) and cyclin/ Cdk inhibitors (CIP/KIP) (Nakayama and Nakayama, 1998; Sherr et al., 1999). These families of CKIs target and inhibit the kinase activity of Cdks via either competing with cyclin binding sites on the Cdks (mechanism of INK) or inhibiting the catalytic site of the cyclin/Cdk complex (mechanism of CIP/KIP) (Canepa et al., 2007; Cayrol et al., 1998).

Although the regulation and mechanisms of cell cycle progression have been well characterized in several mammalian cell lines, the impact of environmental and cellular stresses on cell cycle arrest in mammalian models are still not fully understood. Such 
studies are more common with model organisms like the nematode, Caenorhabditis elegans. For example, harsh environmental conditions that interrupt food availability can trigger cell cycle arrest in C. elegans larvae at either the L1 or L2 stages of development. At the latter stage this causes larvae to exit into a dauer state, a stress-induced diapause characterized by extreme metabolic rate depression (van den Heuvel, 2005). Environmental stress-induced dormancies are common survival strategies across the animal kingdom and in mammals are probably best known as strategies of winter survival including both daily torpor and seasonal hibernation in mammals (Storey and Storey, 2007). One of the hallmarks of metabolic rate depression is the active inhibition of energy-expensive cellular processes. Multiple ATP-consuming processes such as transcription, translation, and ion channel activity are well known to be suppressed during torpor bouts (Storey and Storey, 2007; Storey and Storey, 2010; MacDonald and Storey, 1999; Morin and Storey, 2008).

The present chapter examines the status of cell cycle activity, a highly energyexpensive metabolic function, over six time points of the torpor-arousal cycle in liver and skeletal muscle of I. tridecemlineatus. I hypothesize that during the hypometabolic state of mammalian torpor, markers of cell cycle arrest will be evident in proliferative capable tissues such as the liver, but absent in terminally differentiated skeletal muscle. The induction of cell cycle arrest would function as a mechanism of energy conservation during hibernation. 


\subsection{RESULTS}

\subsubsection{Protein expression of cyclins}

Each phase of cell cycle is regulated by combinations of cyclin and Cdk proteins that form kinase complexes which phosphorylate and regulate many factors crucial for cell division. Hence, the expression of cyclins can be used as a valuable marker when analyzing the relative progression of cell cycle. To determine if cyclin expression oscillates over the torpor-arousal cycle of hibernation, immunoblotting was used to assess relative levels of the four main cyclins $(\mathrm{D}, \mathrm{E}, \mathrm{A}, \mathrm{B})$ over six stages of the torpor-arousal cycle. FIG. 6.2 shows that the protein levels of cyclin D in the liver were suppressed during the EN, LT and IA stages by $32 \%, 31 \%$, and $30 \%$, respectively, as compared to EC $(P<0.05)$. Similarly, the protein expression of cyclin $\mathrm{E}$ also decreased by $51 \%$ and $48 \%$ during EN and LT stages, respectively, compared to EC ( $\mathrm{P}>0.05)$. The expression of cyclin B1 was also reduced by $38 \%$ during the $\mathrm{EN}$, however, along with cyclin $\mathrm{A}$, the expression of cyclin A and cyclin B proteins rose by 1.57 and 2.44-fold respectively during the early arousal stages of the torpor cycle compared to $\mathrm{EC}(\mathrm{P}>0.05)$.

In the skeletal muscle, shown in FIG. 6.3, the protein levels of cyclin D were reduced by $41 \%$ and $64 \%$ during EN and LT respectively, but were elevated by 1.53 -fold in IA when compared to EC $(\mathrm{P}<0.05)$. During ET, levels of both cyclin E and cyclin A increased by 3.35 -fold and 2.42-fold respectively, while cyclin B was lowered by $66 \%$ and $62 \%$ during LT and IA, respectively when compared to $\mathrm{EC}(\mathrm{P}<0.05)$. When comparing between liver and skeletal muscle, only cyclin D showed similar patterns of 
regulation whereas cyclins $\mathrm{E}, \mathrm{A}$, and $\mathrm{B}$ displayed tissue-specific modes of regulation over torpor-arousal.

\subsubsection{Transcriptional profiling of cyclin $m R N A$ expression}

To establish the potential for cyclin regulation at the transcriptional level, RTPCR analysis was conducted to determine the relative gene expression of the four cyclins. FIG.6.4 shows that expression of cyclin D1 was upregulated by 1.51 -fold and 1.58 -fold in liver during EA and LA, respectively, as compared to EC $(\mathrm{P}<0.05)$, while the expression of cyclin $E$ was upregulated by 1.55 -fold during LT compared to EC $(\mathrm{P}<0.05)$. The expression of cyclin A2 showed an upregulation of 1.82-fold, during EA compared to EC, meanwhile, the expression of cyclin B1 remained stable over the torpor-arousal cycle. In the skeletal muscle, shown in FIG. 6.5, mRNA transcript levels of all four cyclins were unchanged over torpor-arousal. Although putative decreases of cyclin E1 and increases of cyclin Dl were observed, neither gene showed statistically different changes due to high variation in amplified product levels between different muscle samples (although expression of the housekeeping gene, $\alpha$-tubulin, was highly consistent between samples)

\subsubsection{Protein expression profiling of cyclin dependent kinases (Cdk)}

To investigate the relationship between cyclin expression and Cdks, the protein expression of four $\mathrm{Cdks}(\mathrm{Cdk} 4, \mathrm{Cdk} 6, \mathrm{Cdk} 2$, and $\mathrm{Cdk} 1)$ that form complexes with cyclins were measured. In the liver analysis shown in FIG. 6.6, the expression of Cdk 4 was reduced by $28 \%$ during ET but increased by 1.21 -fold during EA, as compared to EC $(\mathrm{P}<0.05)$. The expression of Cdk 6 decreased by $33 \%$ during EA compared to EC 
$(\mathrm{P}<0.05)$, whereas the Cdk 1 protein levels were reduced by $29 \%$ and $28 \%$ during EN and IA, respectively, but rose by 1.30 -fold during EA, as compared with $\mathrm{EC}(\mathrm{P}<0.05)$. In skeletal muscle, shown in FIG. 6.7, both Cdk 4 and Cdk 6 protein expression was unchanged over the torpor-arousal course. However, protein expression of Cdk 2 increased by 1.40 -fold in IA, while expression of Cdk 1 decreased by $70 \%$ during LT when compared to $\mathrm{EC}(\mathrm{P}<0.05)$.

\subsubsection{Phosphorylation of checkpoint kinase 1 and 2.}

Apart from cyclins and Cdks, checkpoint kinases act as gatekeepers to regulate the transition from one cell cycle phase to another. Chk 1 and 2 are activated via protein phosphorylation in response to different cellular stress stimuli and subsequently function to phosphorylate cyclin / Cdk complexes to regulate their activity. Shown in FIG. 6.8, the relative phosphorylation level of liver Chk 1 at Ser-296 increased significantly by $2.54-$ fold during EN compared to EC $(\mathrm{P}>0.05)$ but returned to control levels over the remainder of the torpor-arousal cycle. However, phosphorylation of liver p-Chk2 at Thr68 rose later in the cycle being 1.57-fold higher during EA as compared to EC $(\mathrm{P}>0.05)$.

In the skeletal muscle, shown in FIG. 6.9, an increased phosphorylation of Chk 1 at Ser296 was evident in EN by 2.13 -fold compared to $\mathrm{EC}(\mathrm{P}<0.05)$, similar to the pattern observed in liver. Relative phosphorylation of Chk 2 at Thr-68 was unchanged over the six stages of the torpor-arousal cycle in muscle.

\subsubsection{Protein expression of cell cycle inhibitors p15INK4b and $p 21 C I P 1$}

One of the key mechanisms in eukaryotic cell cycle arrest is the action of cell cycle inhibitors that disrupt the cyclin-Cdk complex. These CKIs are categorized into 
distinct two families, INK4 and CIP/KIP. To determine if CKIs are regulated during hibernation, expression levels of p15INK4b and p21CIP1 were evaluated. As shown in FIG. 6.10, relative protein levels of p15INK $4 b$ in liver increased significantly by 1.40 fold in ET compared to EC ( $\mathrm{P}>0.05)$. Protein expression of liver p21CIP1 also rose, with an increase of 1.37-fold during LT compared to EC ( $\mathrm{P}>0.05)$, but was then strongly reduced to $47 \%$ of the EC value in the IA stage. In skeletal muscle, shown in FIG. 6.11, the protein expression of $\mathrm{p} 15 \mathrm{INK} 4 \mathrm{~b}$ did not change significantly over the torpor-arousal cycle. However, p $21 \mathrm{CIP} 1$ protein levels were strongly reduced by $33 \%, 70 \%$, and $80 \%$ during EN, LT, and IA, respectively, as compared to EC but rose by 1.49 -fold during ET $(\mathrm{P}<0.05)$

\subsubsection{Regulation of PCNA and phosphorylated p 21}

In addition to targeting cyclins and Cdks, p21CIP1 has previously been shown to play a role in cell cycle arrest through interactions with proliferating cell nuclear antigen (PCNA). PCNA functions as a $\beta$ loading clamp, a crucial component of the DNA replication complex that is often used as a marker for the synthesis phase of the cell cycle. The phosphorylation of p21CIP1 at Ser-146 regulates the cellular translocation of the protein, as well as regulating its interaction with PCNA. To assess the role of p21CIP1 in PCNA regulation, PCNA protein levels were measured along with the phosphorylation state of p-p21CIP1 at Ser-146. FIG. 6.12 shows that phosphorylation of liver p-p21CIP1 at Ser-146 increased strongly by 2.75-fold, 4.56-fold, and 3.76-fold during EN, EA, LA compared to EC respectively $(\mathrm{P}>0.05)$. By contrast, the expression of liver PCNA was strongly reduced to $48 \%$ of the EC value during the LT stage of 
torpor. In skeletal muscle, shown in FIG. 6.13, relative phosphorylation of p21CIP1 at Ser-146 increased by 2.46-fold during EA as compared to EC $(\mathrm{P}<0.05)$. Meanwhile, protein expression of PCNA decreased in EN by $65 \%$ but rebounded during LT and IA to levels 1.58 -fold and 1.70-fold higher than the EC value $(\mathrm{P}<0.05)$.

\subsubsection{Partial cDNA cloning of squirrel cyclin transcripts}

PCR analysis of cyclin D1 yielded an amplicon of 344 bp in length with a translated amino acid sequence with length of 114 (FIG. 6.14). When compared to other mammals, ground squirrel cyclin D1 showed high homology with the sequence from other mammals with percent conservation values between the squirrel sequence with mouse (89.2\%), rat (89.2\%), and human (89.6\%) (FIG. 6.15).

The PCR analysis of cyclin E1 yielded an amplicon of $306 \mathrm{bp}$ in length that closely matched the sequence of cyclin E1 from other mammals. The nucleotide sequence of the amplicon translated into a peptide sequence of 109 amino acids (FIG. 6.16). When compared to other mammals, as shown in FIG. 6.17., the homology tree showed a relatively high degree of conservation between the squirrel sequence and the mouse (85.1\%), rat (85.4\%), and human $(86.8 \%)$ sequences.

The PCR analysis of cyclin A2 yielded an amplicon of $322 \mathrm{bp}$ in length. The nucleotide sequence translated into an amino acid sequence with length of 107 (FIG. 6.18). When compared to other mammals, as shown in FIG. 6.19, the homology tree produced relatively high degree of conservation between the squirrel sequence to mouse (89.7\%), rat (90.5\%), and human $(92.0 \%)$. 
The PCR analysis of cyclin B1 yielded an amplicon of $78 \mathrm{bp}$ in length. The nucleotide sequence of the amplicon was translated to yield a peptide sequence of 26 amino acids (FIG. 6.20). When compared to other mammals, as shown in FIG. 6.21., the homology tree produced relatively high degree of conservation between the squirrel sequence to mouse (85.1\%), rat (85.4\%), and human $(86.8 \%)$.

\subsection{DISCUSSION}

Although division of hepatocyte cells in adult rodents is infrequent, the proliferative capacity of liver is always maintained and allows the organ to regenerate tissue mass through hepatocyte cell division in response to tissue damage (Taub, 2004). The cell cycle is the major process that is responsible for regulating tissue growth and division, and is activated upon stimulation from extracellular signals such as growth factors and mitogens (Obaya and Sedivy, 2002; Bottazzi and Assoian, 1997; Jones and Kazlauska, 2000). By contrast, adult skeletal muscle is considered to be terminally differentiated, with cells permanently in engaged the $\mathrm{G}_{0}$ state. In the present chapter, we show that cell cycle progression displays a tissue specific pattern between liver and skeletal muscle that reflects the fundamental cell division capabilities of the two tissues.

\subsubsection{Regulation of cyclins and CDKs in liver}

Cell cycle progression is an expensive process and is dependent on plentiful ATP provided by aerobic metabolism in mitochondria (Mandal et al., 2005). Previous studies with Drosophila showed that a $60 \%$ reduction in mitochondrial ATP production was sufficient to cause cell cycle arrest, mediated by a downregulation of cyclin E (Mandal et 
al., 2005). The hibernation season is characterized by cycles of profound metabolic rate depression, often to levels $<5 \%$ of resting metabolic rate in euthermia. Such strong metabolic suppression argues that cell cycle activity should be strongly inhibited in parallel with the known global suppression of multiple other ATP-expensive cell functions during torpor including the global suppression of transcription and translation that are a very necessary part of cell division (Storey and Storey, 2007; Storey and Storey, 2010; MacDonald and Storey 1999; Morin and Storey, 2008). Similar to cell cycle arrest in Drosophila (Mandal et al., 2005), both cyclin D1 and cyclin E protein expression decreased in liver when squirrels entered torpor (EN) and were also reduced in prolonged torpor (LT) (FIG. 6.2). The decrease in cyclin D1 protein expression was not correlated with changes in cyclin D1 mRNA expression (FIG. 6.4). A similar disjoint between cyclin D1 mRNA and protein levels was seen during anoxia-induced hypometabolism in turtle liver and was linked with microRNA suppression of cyclin D1 transcript expression (Biggar and Storey, 2012). Whether this might also occur in aerobic torpor during hibernation remains to be explored. During ET a suppression of Cdk 4 also occurred (FIG. 6.6). The decrease in both cyclin D1 and its binding partner Cdk 4 suggests that progression through the $\mathrm{G}_{1}$ phase of the cell cycle is negatively regulated during the torpor stages of hibernation. As previously mentioned, the cyclin D1:Cdk 4 complex acts as a positive regulator of cell cycle progression by promoting the expression of many S phase regulators including expression of cyclin E, cyclin A, and Cdc25 genes (Ohtani and Nevins, 1994; Johnson and Walker, 1999). Interestingly, we observed an increase in cyclin E mRNA during LT, although cyclin E protein levels were reduced. Although this inverse correlation remains unexplained, the decrease in cyclin E 
protein expression suggests that the progression from $\mathrm{G}_{1}$ to $\mathrm{S}$ phase is stalled during late torpor. Since the expression of cyclin D1 has previously been shown to be regulated by the mTORC1 signaling pathway (Averous et al., 2008), the suppression of cyclin D1 expression during LT also fits with the comparable suppression of the mTORC1 signaling network that was previously reported by McMullen and Hallenbeck (2010). A decrease in translational capacity due to mTOR inhibition during LT could account for the decrease in cyclin D1 expression, and in turn, help to stall cell cycle progression at the $\mathrm{G}_{1} \rightarrow \mathrm{S}$ transition stage. In addition, FIG 6.2 shows a strong suppression of both cyclin D1 and cyclin E protein levels during the entrance phase into torpor, suggesting that the progression towards an arrested cell cycle is initiated during the time when the animal is actively suppressing its metabolic rate and $\mathrm{Tb}$ as it descends into torpor.

Interestingly, beyond the $\mathrm{G}_{1} / \mathrm{S}$ checkpoints, a different pattern of expression emerged for regulators of the S phase and the $\mathrm{G}_{2} \rightarrow \mathrm{M}$ phase transition. Cyclin A is a well-known regulator of S phase when it is complexed with $\mathrm{Cdk} 2$ and its expression has been correlated with DNA synthesis during S phase (Vermeulen et al., 2003; Girad et al., 1991). Both cyclin A protein and mRNA expression levels were stable during entrance into and throughout torpor as was Cdk 2 expression during EN (FIG. 6.2, 6.4, and 6.6). These data confirm that the $\mathrm{G}_{2}$ phase of the cell cycle is not differentially regulated during EN and the torpor stages of the hibernation. However, when ground squirrels started to arouse (EA) cyclin A transcripts and protein increased sharply and transcript levels remained elevated during interbout (IA) (FIG. 6.2, 6.4). This indicates a renewal of S phase activity when the animals arouse out of torpor. 
The progression from $\mathrm{G}_{2}$ to $\mathrm{M}$ phase is regulated by the cyclin $\mathrm{B} 1$ and $\mathrm{Cdk} 1$ complex; this complex is required and its expression rises dramatically as cells enter the $\mathrm{G}_{2} / \mathrm{M}$ boundary (Arellano and Moreno, 1997). As seen in FIG.6.2, a suppression cyclin B1 protein levels was observed during EN but levels were not different from controls during either ET or LT. However, cyclin B1 increased greatly when the animals were arousing in EA although this was not supported by elevated cyclin B1 transcript levels. Expression of Cdk 1 mimicked the responses of cyclin B1, with a reduction during EN followed by a strong upregulation during EA (FIG. 6.6). Hence, both cyclin A and B1 were strongly upregulated during arousal from torpor. These data support the conclusion that the $\mathrm{G}_{1} / \mathrm{S}$ arrest observed during the torpor stages is reversed during arousal, when the animal re-initiates metabolic processes to restore euthermia. This overall pattern of cyclin expression during hibernation suggests that the decreases in cyclin D1 and cyclin E expression induces a $\mathrm{G}_{1} / \mathrm{S}$ phase arrest during torpor; moreover, the elevated expression of cyclin $A$ and cyclin $B 1$ suggests the $G_{1} / S$ phase arrest is reversed when animals begin to arouse back to euthermia.

\subsubsection{Regulation of cyclins and CDKs in skeletal muscle}

Once matured, skeletal muscle is terminally differentiated and subsequent changes in muscle mass are primarily regulated by a balance of anabolic and catabolic activities that remodel myocytes rather than proliferate new cells (Stitt et al., 2004). Due to the restricted functions of skeletal muscle during hibernation, evidence of muscular atrophy suggests that metabolic processes are heavily suppressed during hibernation (Wickler, 1991). However, it is fascinating to see how the components of the cell cycle 
are regulated in hypometabolic tissues that do not undergo cell division. Interestingly, the expression of skeletal muscle cyclins seen in FIG 6.3 displayed some regulatory patterns similar to those of liver. A decrease in cyclin D protein content was observed during entry and late stages of torpor, identical to that seen in liver; however, an elevation of cyclin E and cyclin A was seen during ET, and no evidence of elevated cyclin A nor B was observed in the arousal stages of hibernation. When examining the regulation of cyclins at the mRNA level, it was surprising that all four cyclins showed no significant changes into their transcript expression throughout the torpor-arousal cycle (FIG. 6.5). This was also somewhat evident in the regulation of CDK proteins, where minimal changes were observed during the hibernation cycle (FIG. 6.7).

The stable expression of cyclin mRNA and Cdk protein levels in skeletal muscle might suggest that the changes observed in cyclin proteins over the torpor-arousal cycle could regulate other cellular processes, providing functions outside of their canonical role of cell cycle regulation. Indeed, previous studies have shown that the cyclin D - Cdk4 complex functions to regulate myocyte enhancer factor (MEF) family proteins, whose function as transcription factors regulate muscle growth and metabolism. Indeed, Lazaro et al. (2002) showed that cyclin D-Cdk 4 activity blocks the interaction of MEF2C with co-activators, and inhibits the transcriptional activity of MEF2. The protein expression and phosphorylation of MEF2A and MEF2C has previously been shown to be elevated during the torpor stages of hibernation, and this was coupled with increased transcriptional activity (Tessier and Storey, 2010). It is possible that the decrease in cyclin D protein expression observed functions to support the activation of MEF2 protein, and aids in the regulation of muscle metabolism during torpor. 


\subsubsection{Sequence analysis of cyclins}

Using nucleotide sequence information derived from PCR amplicons (FIG. 6.14, 6.16, 6.18, and 6.20), partial amino acid sequences of ground squirrel cyclins were derived and compared with three other mammalian species (FIG. 6.15, 6.17, 6.19, and 6.21). Based on the amino acid alignments, all four ground squirrel cyclin proteins displayed high homology with the sequences from non-hibernating mammals (mouse, rat, human). Cyclin A showed the highest degree of homology, with conservation ranging between $89.7 \%$ - 92.0\% whereas cyclin E displayed the lowest degree of homology, with a conservation range of $86.3 \%-98.5 \%$. The high degree of amino acid similarity observed suggests that the structure and functions of cyclin proteins are likely to be conserved and function similarly to those described in other mammalian systems.

\subsubsection{Regulation of checkpoint kinases}

Although the oscillating patterns of cyclin proteins are often good indicators of cell cycle progression, complex regulatory systems also exist that control the activity of cyclin/Cdk complexes. Most notably, cyclin/Cdk complexes are tightly regulated by check point kinases (Chk) and CKIs. Chk 1 and Chk 2 are regulated and activated via phosphorylation by ataxia telangiectasia and $\operatorname{rad} 3$ related (ATR) and ataxia telangiectasia mutated (ATM) proteins, two stress response protein kinases that are triggered by UV or IR induced DNA damage (Abraham, 2001). As seen in FIG. 6.8 and FIG. 6.9, the relative amount of phosphorylated Chk 1 at Ser-296 was elevated strongly during entrance into torpor in both liver and skeletal muscle, whereas p-Chk 2 (Thr-68) content increased during arousal from torpor only in the liver. The increase in Chk 1 
phosphorylation during entrance in both tissues is interestingly, since Ser-296 is phosphorylated in response to DNA damage, and functions as a gatekeeper of the $\mathrm{G}_{2} / \mathrm{M}$ checkpoint (Okita et al., 2012; Kasahara et al., 2010). Hence, increased phosphorylation of this residue could be evidence of cellular damage in the entry phase of hibernation, when animals experience the initial decrease in metabolic functions and $\mathrm{Tb}$. Interestingly, although evidence of $\mathrm{G}_{2} / \mathrm{M}$ arrest was not present in either liver nor skeletal muscle, the activation of Chk-1 could function to phosphorylate other downstream targets, such as tumour protein 53 (p53), which functions to activate stress responsive pathways via regulating DNA repair and apoptosis (Levine and Oren, 2009).

\subsubsection{Regulation of cell cycle inhibitors (CKI)}

CKIs are considered the 'brakes' on the cell cycle engine and are divided into two distinct groups, the INK4 family and the CIP/KIP family (Nakayama and Nakayama, 1998; Sherr and Roberts, 1999). The present study examined the mRNA and protein expression of two well-studied CKIs, p15INK4b and p21CIP1. The INK4 proteins bind specifically to the $\mathrm{G}_{1} \mathrm{Cdks}(\mathrm{Cdk} 4$ and Cdk6) and inhibit Cdk associations with D-type cyclins, promoting a $\mathrm{G}_{1}$ arrest (FIG. 6.22A) (Cánepa et al., 2007). Protein levels of p15INK4b increased significantly during early torpor in the liver, but remained unchanged in skeletal muscle throughout the torpor-arousal cycle (FIG 6.10, 6.11). Previous studies implicated a role of p15INK4b in establishing cell cycle arrest and a substantial increase in expression of p15INK $4 \mathrm{~b}$ was found in senescent chicken cells (Kim et al., 2006). Since p15INK4b is known to target CDK4/6 exclusively, its upregulation during torpor, coupled with the suppression of D-type cyclins in the liver, 
suggests that the $G_{1}$ stage of the cell cycle is strongly targeted for suppression during torpor in liver. The upregulation of $\mathrm{p} 15 \mathrm{INK} 4 \mathrm{~b}$ expression during torpor is plausible, since its expression has previously been shown to be regulated by transforming growth factor- $\beta$ (TGF- $\beta$ )/ SMAD signaling, a cellular cascade regulated by anti-mitogenic cytokines (Shen et al., 1998), that is activated during torpor (see Chapter 5). Hence, the torpor phase appears to be the time when $\mathrm{p} 15 \mathrm{INK} 4 \mathrm{~b}$ effects on cell cycle regulation are most important.

The CIP/KIP proteins inhibit the enzymatic complex formed by the cyclin and Cdk proteins via interactions with both the regulatory subunit of the cyclin and the catalytic subunit of Cdk (FIG. 6.22B) (Cayrol et al., 1998). FIG. 6.10 and 6.11 shows that the protein expression of $\mathrm{p} 21 \mathrm{CIP} 1$ increased significantly during LT in liver, whereas it only increased during ET and was reduced during EN, LT and IA in muscle. The inhibitory action of p21CIP1was previously shown to preferentially target Cdk 2 complexes, suggesting that $\mathrm{p} 21 \mathrm{CIP} 1$ primarily inhibits the cyclin E/Cdk 2 and cyclin A/Cdk 2 complexes that are responsible for regulating the $G_{1} \rightarrow S$ transition and $S$ phase of the cell cycle, respectively (Vidal and Koff, 2000). Similar to p15INK4b, p21CIP1 has also been linked to induction of a senescent state, as suggested by its alias: p21Sdi1 or the senescent cell-derived inhibitor (Kagawa et al., 1999). Studies by Kagawa et al. (1999) observed the induction of a cellular senescence phenotype in cancer cells via $G_{1}$ cell cycle arrest initiated by the overexpression of $\mathrm{p} 21$ Sdi1. When comparing the two types of CKI, it can be seen that their protein expression levels are elevated at distinct stages of torpor in the liver. FIG. 6.10 shows that enhanced expression of p15INK4b was observed during ET, followed by increased expression of p21CIP1 during LT. These findings 
suggest that a sequential suppression of cell cycle progression by CKIs occurs and parallels the progression of the hibernation cycle, where a p15INK $4 b$ mediated $\mathrm{G}_{1}$ arrest takes place (or precedence) during ET, followed by a p21CIP1 mediated G1/S arrest during LT in the liver.

In addition to inhibition of Cdks, p21CIP1 has also been shown to induce cell cycle arrest via interactions with PCNA, an essential subunit of DNA polymerase $\delta$ (Cayrol et al., 1998). PCNA functions as a DNA sliding clamp that is required for chromosomal DNA synthesis, and has previously been described as a S-phase marker in Lytechinus pictus and C. elegans embryonic cells (Kisielewska et al., 2005). Previous studies showed that phosphorylation of p21CIP1 at Ser-146 strongly inhibited its binding ability with PCNA through interference with the intermolecular hydrogen bonds that anchor the two proteins (Scott et al., 2000). As seen in FIG. 6.12 for liver, the relative phosphorylation state of p21CIP1 at Ser-146 increased during entrance into torpor, was reduced during torpor, but then rose dramatically during arousal (EA) and remained high during interbout. By contrast, PCNA expression was reduced during LT. Shown in FIG 6.13 for the skeletal muscle, p-p21CIP1 (Ser-146) levels were increased during EA, similar to liver; however, expression of PCNA was elevated during ET and IA. The decrease in this S-phase marker in liver further supports the proposal of S phase arrest during torpor. In addition, the dramatic increase ( $>3$-fold) in p-p21CIP1 content during arousal indicates reduced inhibition of PCNA by $\mathrm{p} 21 \mathrm{CIP} 1$ at this time. This observation, along with the previously described elevation of cyclin A and cyclin B1 protein expression during the arousal stages, supports the idea of a reversal of $\mathrm{G}_{1} / \mathrm{S}$ arrest when ground squirrels exit torpor. However, in the skeletal muscle, it is unclear why PCNA 
was upregulated during torpor, since it primarily functions as a cofactor of DNA polymerase during DNA replication.

\subsection{CONCLUSION}

The present study examined the molecular mechanisms that control cell cycle progression in ground squirrel liver and skeletal muscle during hibernation. This study demonstrated that liver, but not skeletal muscle displayed markers of cell cycle arrest, and that the cellular proteins that regulate the $G_{1}$ and $G_{1} \rightarrow S$ phase are targeted for suppression during torpor to bring about arrest. In the liver, the data show that D- and Etype cyclins were suppressed during late torpor, which could decrease Cdk activities in the initial phases of the cell cycle. The patterns of p15INK4 and p21CIP1 overexpression suggested that CKIs are critical regulators of cell cycle suppression during torpor. Both $\mathrm{p} 15 \mathrm{INK} 4 \mathrm{~b}$ and $\mathrm{p} 21 \mathrm{CIP} 1$ have been implicated in the induction of cellular senescence, but in the hibernator the expression pattern of both proteins returned to near euthermic (EC) values during arousal from hibernation, exhibiting a dramatic reduction in expression when compared to the torpor stages. This shows that the elevation of both proteins during torpor observed in the liver is reversed as animals initiate arousal. The reduction in CKI expression during arousal, coupled with the increase in cyclin A and cyclin B1 proteins at the same time, suggests that cell cycle progression is re-initiated in liver when ground squirrels exit torpor and rewarm back to euthermia. These observations suggest that senescence (a state of irreversible cell cycle arrest) does not occur during torpor. More importantly, the reversible nature of cell cycle regulation in ground squirrels agrees with a cardinal feature of cellular quiescence which is defined as a state of reversible growth 
and proliferation arrest (Coller et al., 2006). In skeletal muscle, however, cyclin proteins appear to be the only class of cell cycle regulators that undergo significant regulation over the torpor-arousal cycle. Although skeletal muscle cells do not undergo active cell division, the observed pattern of cyclin regulation could function outside of their canonical pathways, and regulate other aspects of muscle metabolism.

The hibernation cycle requires a global suppression of energy-expensive cellular functions to promote survival; for example, previous studies have shown a decrease in Erk1/2 expression and MAPKAPK-2 activity during torpor in liver of Richardson's ground squirrels, suggesting a probable decrease in growth factor and mitogen signaling during hibernation (MacDonald and Storey, 2005). Consequently, the changes in cell cycle related proteins observed in this study could be a response to the changes in levels of signalling molecules that take place during torpor. As a result, regardless of the state of cell cycle progression, it is possible that the components of the cell cycle machinery are naturally suppressed as animals enter hypometabolism, as exemplified by cyclin regulation in skeletal muscle. In conclusion, the present findings describe torpor as a state of cellular quiescence in ground squirrel liver during the hibernating season. 


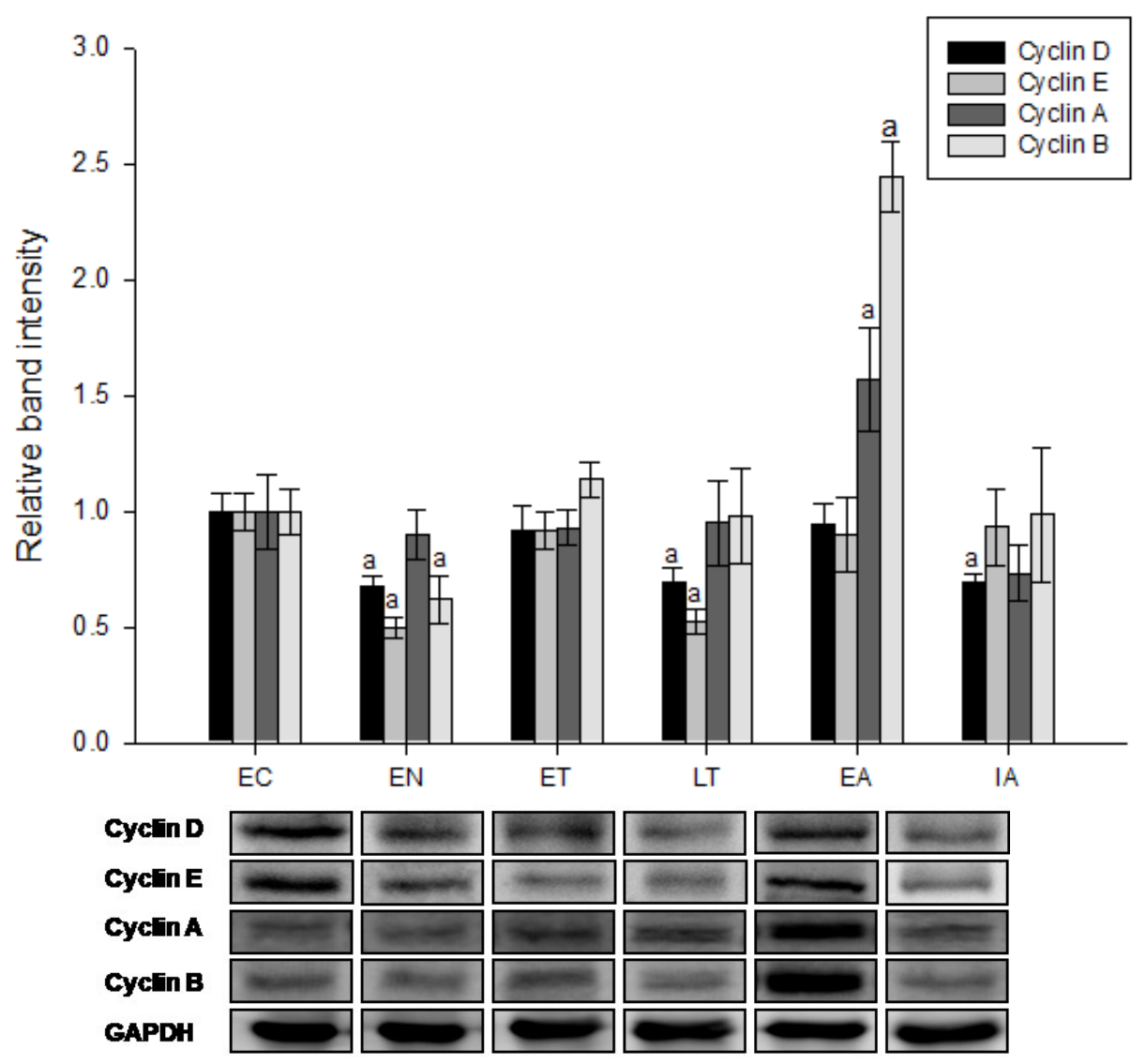

FIG. 6.2. Expression pattern of cyclin proteins in liver of I. tridecemlineatus over the torpor-arousal cycle as determined by Western immunoblotting.

Relative expression of cyclin D, cyclin E, cyclin A, cyclin B, and GAPDH protein over the torpor-arousal cycle in the liver of the 13-lined ground squirrel. Bands on Western blots were detected for cyclin D1 at $\sim 36 \mathrm{kDa}$, cyclin E1 at $\sim 56 \mathrm{kDa}$, cyclin A2 at $\sim 55 \mathrm{kDa}$, cyclin $\mathrm{B} 1$ at $\sim 58 \mathrm{kDa}$, and the housekeeping protein GAPDH was detected at $\sim 32 \mathrm{kDa}$. Data are means \pm S.E.M., $\mathrm{n}=4-5$ independent trials on tissue from different animals. EC, Euthermic control; EN, early entrance into torpor; ET, early torpor; LT, late torpor; EA, early arousal; IA, interbout arousal. a-Significantly different from the corresponding EC value ( $\mathrm{P}<0.05$, ANOVA with post hoc Holm-Sidak test). 

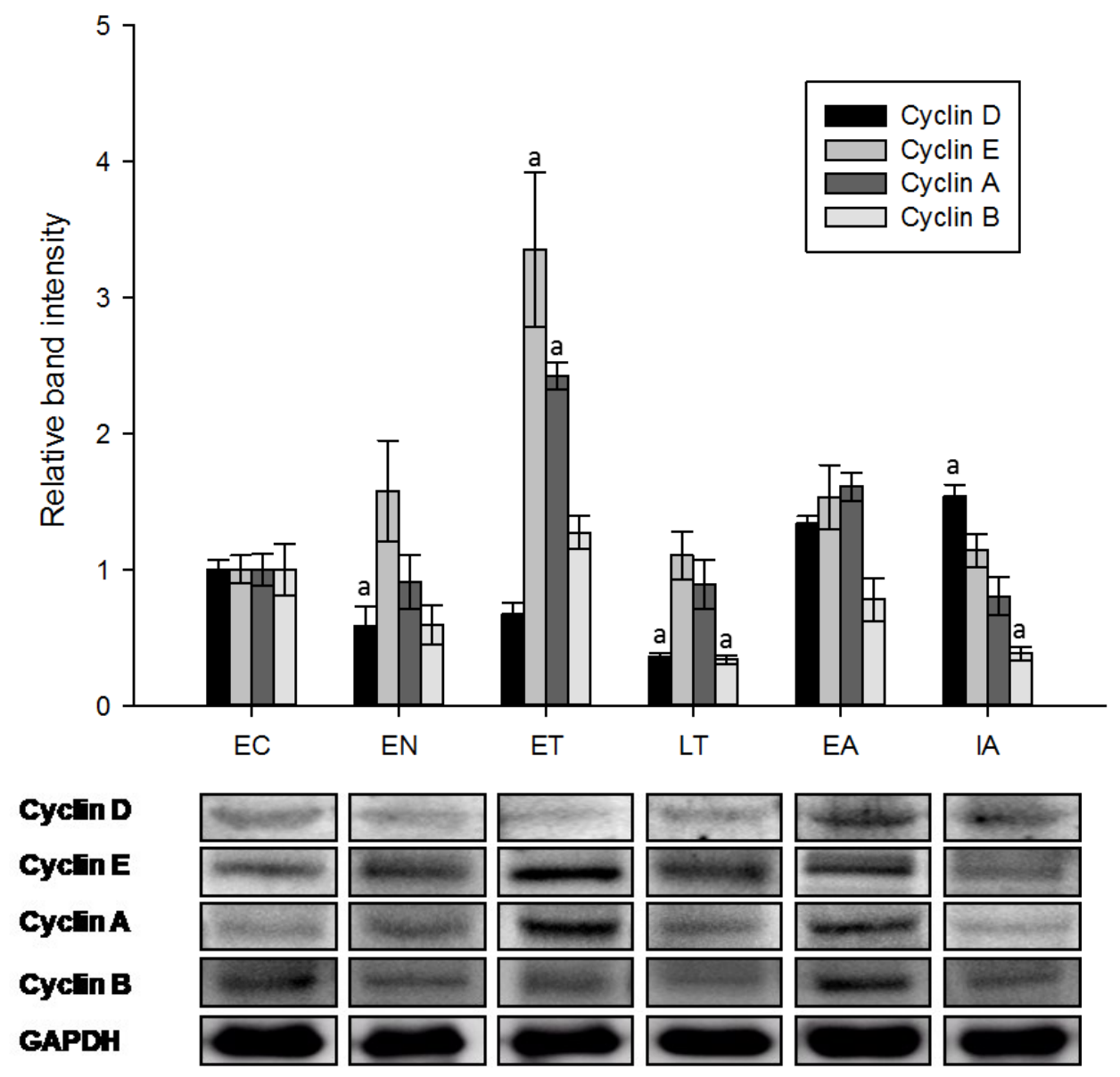

FIG. 6.3. Regulation pattern of cyclin proteins in skeletal muscle of $\boldsymbol{I}$.

tridecemlineatus as determined by Western immunoblotting.

Relative expression of cyclin D, cyclin E, cyclin A, cyclin B, and GAPDH protein over the torpor-arousal cycle in liver of the 13-lined ground squirrel. Representative Western blot bands are shown for cyclin D1, cyclin E1, cyclin A2, cyclin B1, and the housekeeping protein GAPDH. Data are means \pm S.E.M., $n=4-5$ independent trials on tissue from different animals. a-Significantly different from the corresponding EC value $(\mathrm{P}<0.05$, ANOVA with post hoc Holm-Sidak test). 

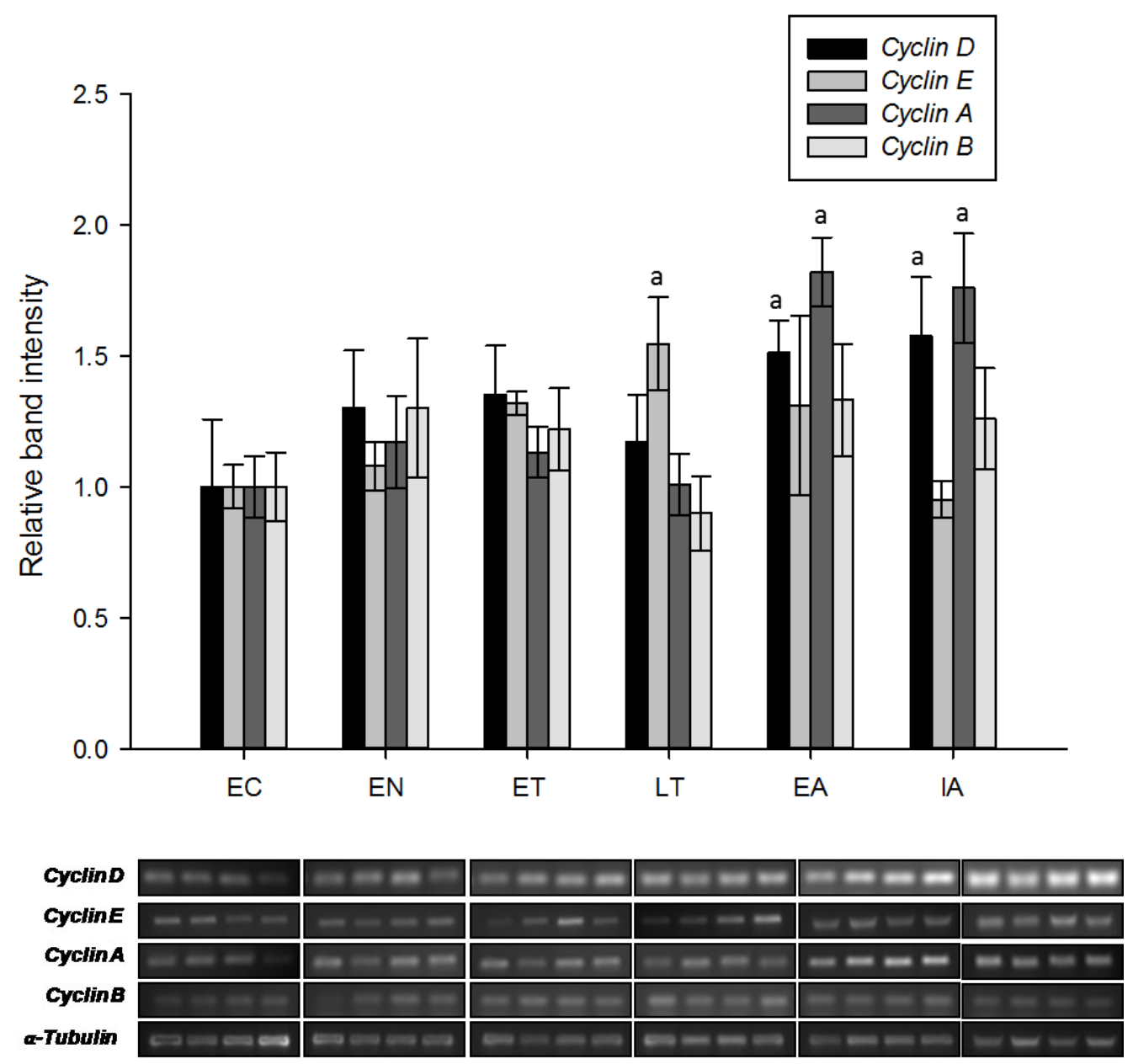

FIG. 6.4. Transcriptional regulation of cyclin mRNA in liver of I. tridecemlineatus as determined by RT-PCR.

Effects of hibernation on cyclin mRNA transcript levels in the liver of 13-lined ground squirrels over the torpor-arousal cycle. SyBR green stained agarose gels are shown for cyclin D1, cyclin E1, cyclin A2, cyclin B1 genes and for the housekeeping gene, $\alpha$-tubulin. Data are means \pm S.E.M., $\mathrm{n}=4$ independent trials on tissue from different animals. a-Significantly different from the corresponding euthermic values $(\mathrm{P}<0.05$, ANOVA with post hoc Holm-Sidak test). 

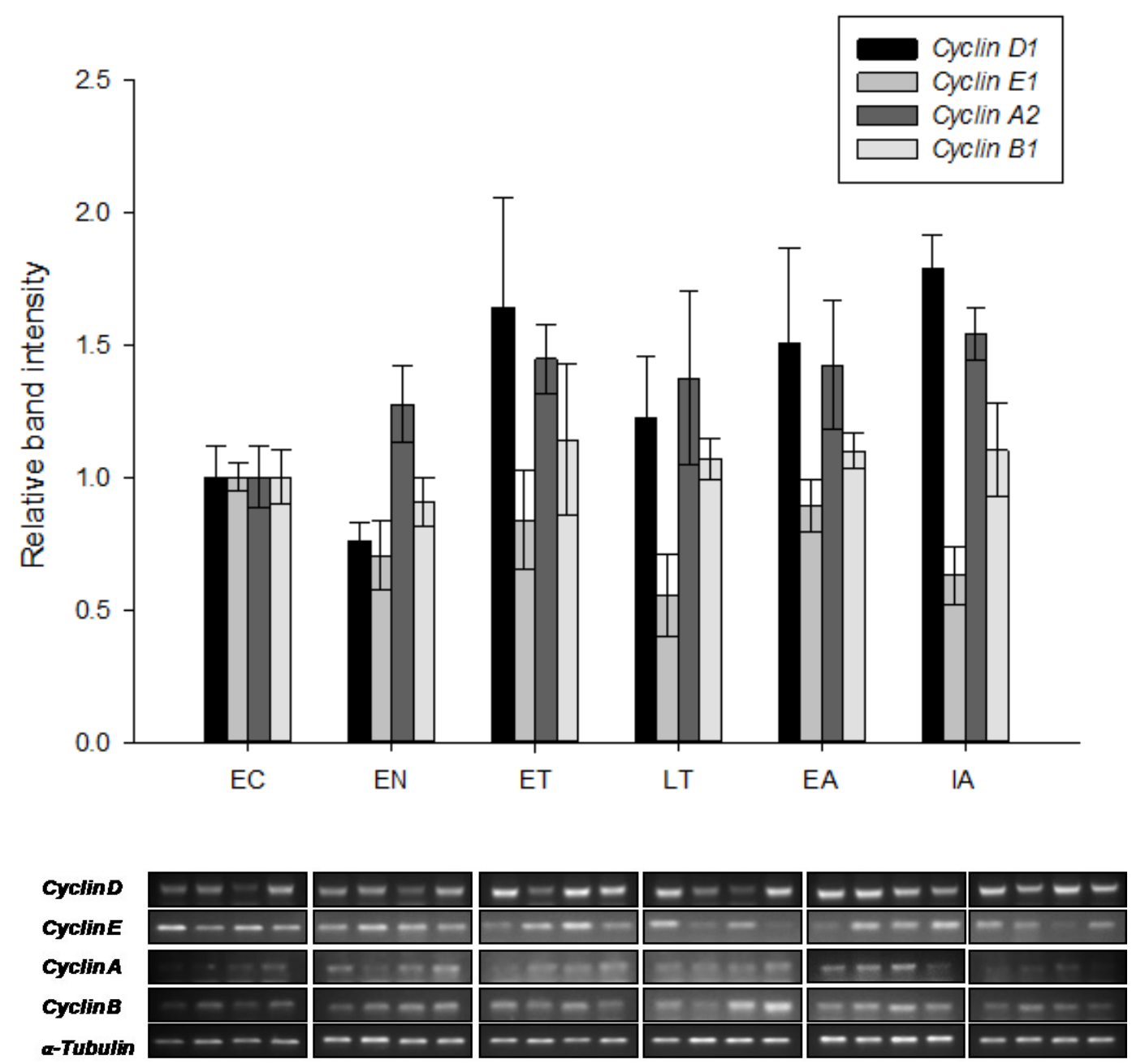

FIG. 6.5. Transcriptional regulation of cyclin mRNA in skeletal muscle of $I$. tridecemlineatus as determine by RT-PCR.

Effects of hibernation on cyclin mRNA transcript levels in the skeletal muscle of 13-lined ground squirrel during the stages of the torpor-arousal cycle. SyBR green stained agarose gels are shown for cyclin D1, cyclin E1, cyclin A2, cyclin B1 genes and for the housekeeping gene, $\alpha$-tubulin. Data are means \pm S.E.M., $\mathrm{n}=4$ independent trials on tissues from different animals. 


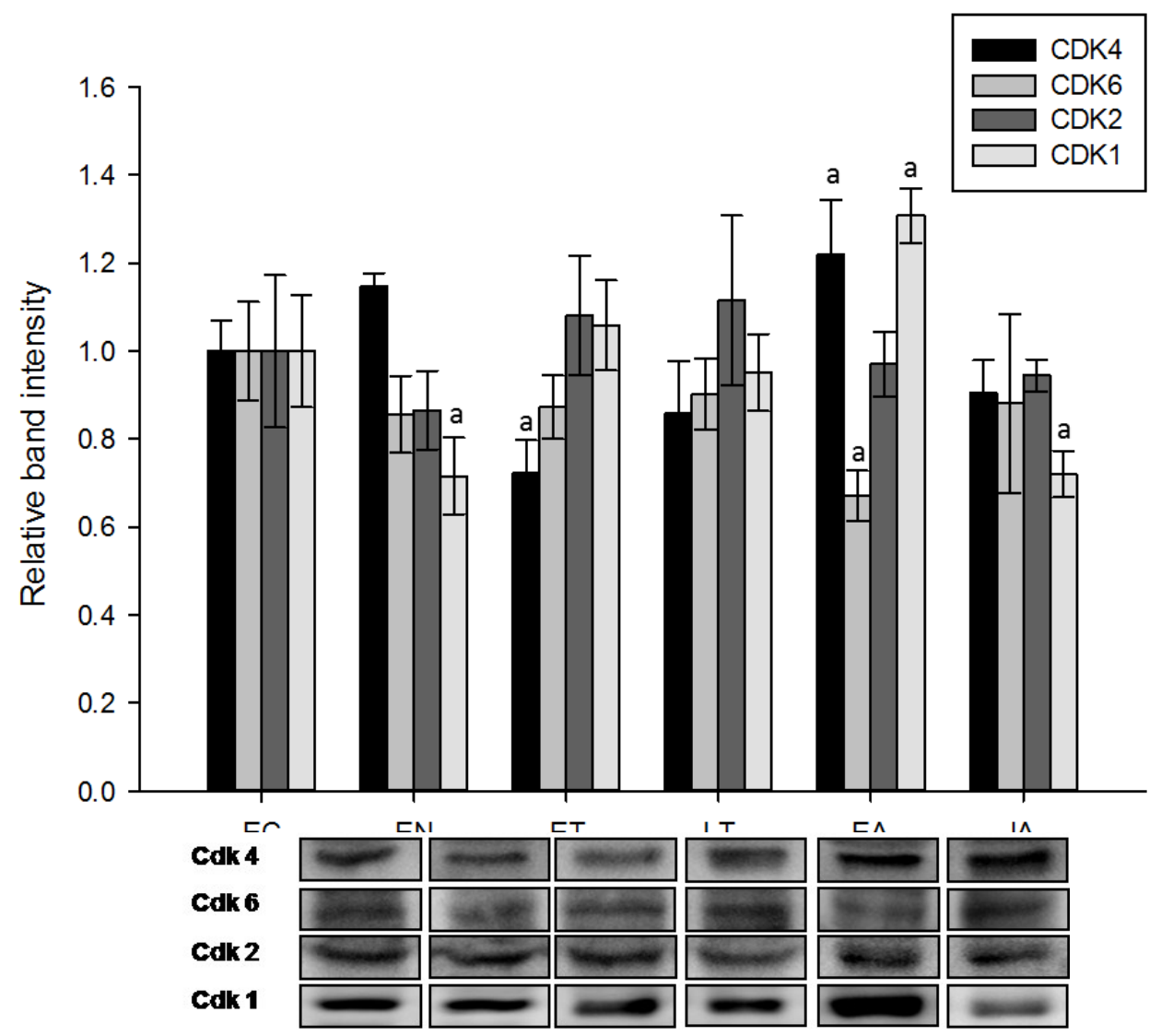

FIG. 6.6. Protein levels of CDKs in liver of I. tridecemlineatus as determined by Western immunoblotting.

Relative protein levels of $\mathrm{Cdk} 4, \mathrm{Cdk} 6, \mathrm{Cdk} 2$, and $\mathrm{Cdk} 1$ over the torpor-arousal cycle in the liver of the 13-lined ground squirrel. Bands on Western blots were detected for Cdk4 at $\sim 30 \mathrm{kDa}, \mathrm{Cdk} 2$ at $\sim 33 \mathrm{kDa}, \mathrm{Cdk} 6$ at $\sim 36 \mathrm{kDa}$, and Cdk 1 at $\sim 34 \mathrm{kDa}$. Data are means \pm S.E.M., $\mathrm{n}=4-5$ independent trials on tissues from different animals. a-Significantly different from corresponding EC values $(\mathrm{P}<0.05$, ANOVA with post hoc Holm-Sidak test). 


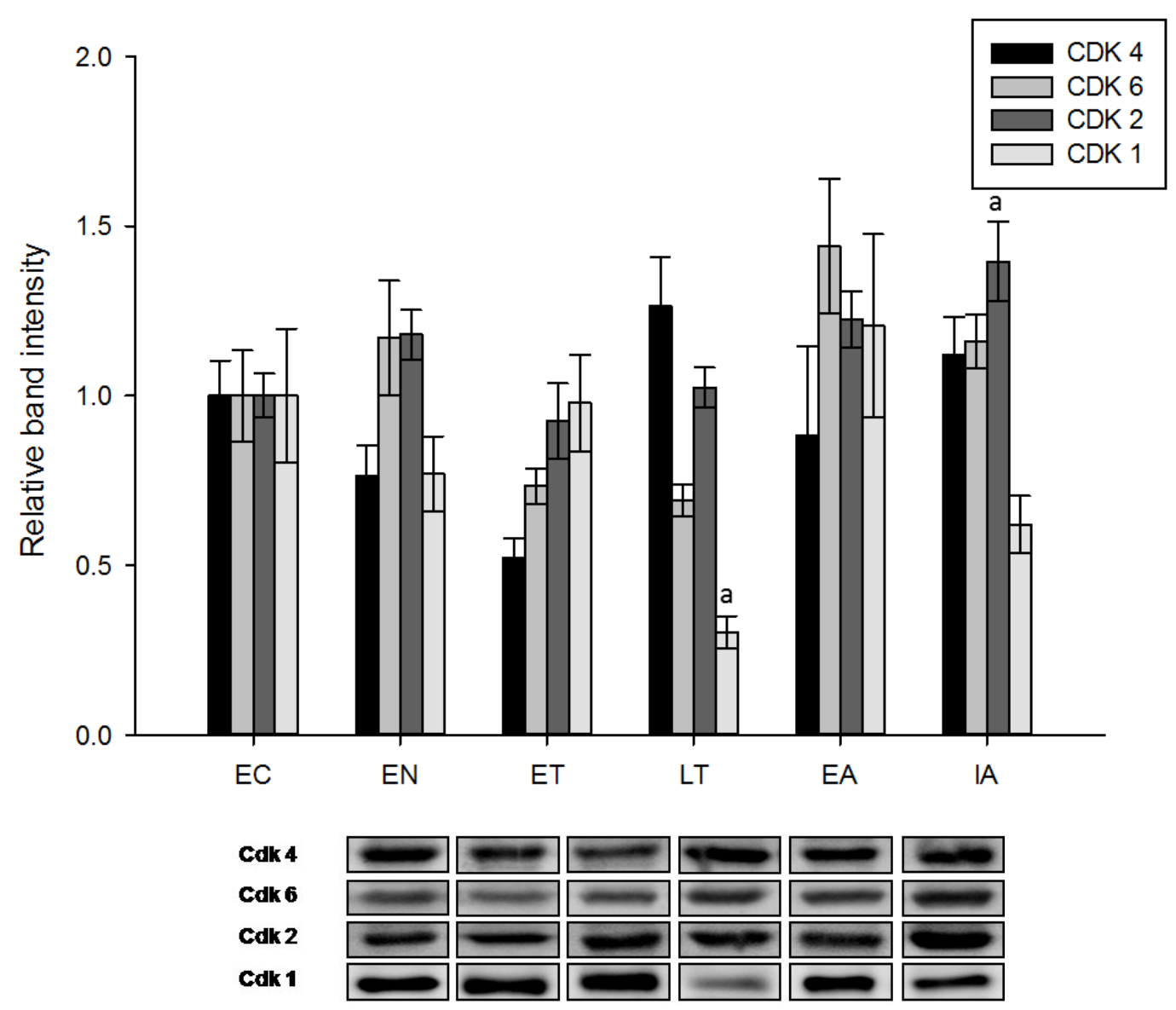

FIG. 6.7. Protein levels of CDKs in skeletal muscle of I. tridecemlineatus as determined by Western immunoblotting.

Relative expressions of $\mathrm{Cdk} 4, \mathrm{Cdk} 6, \mathrm{Cdk} 2$, and $\mathrm{Cdk} 1$ protein over the torporarousal cycle in the skeletal muscle of the 13-lined ground squirrel. Representative Western blot bands are shown for Cdk 4, Cdk 2, Cdk 6, and Cdk 1. Data are means \pm S.E.M., $\mathrm{n}=4-5$ independent trials on tissues from different animals. a-Significantly different from corresponding EC values $(\mathrm{P}<0.05$, ANOVA with post hoc Holm-Sidak test). 


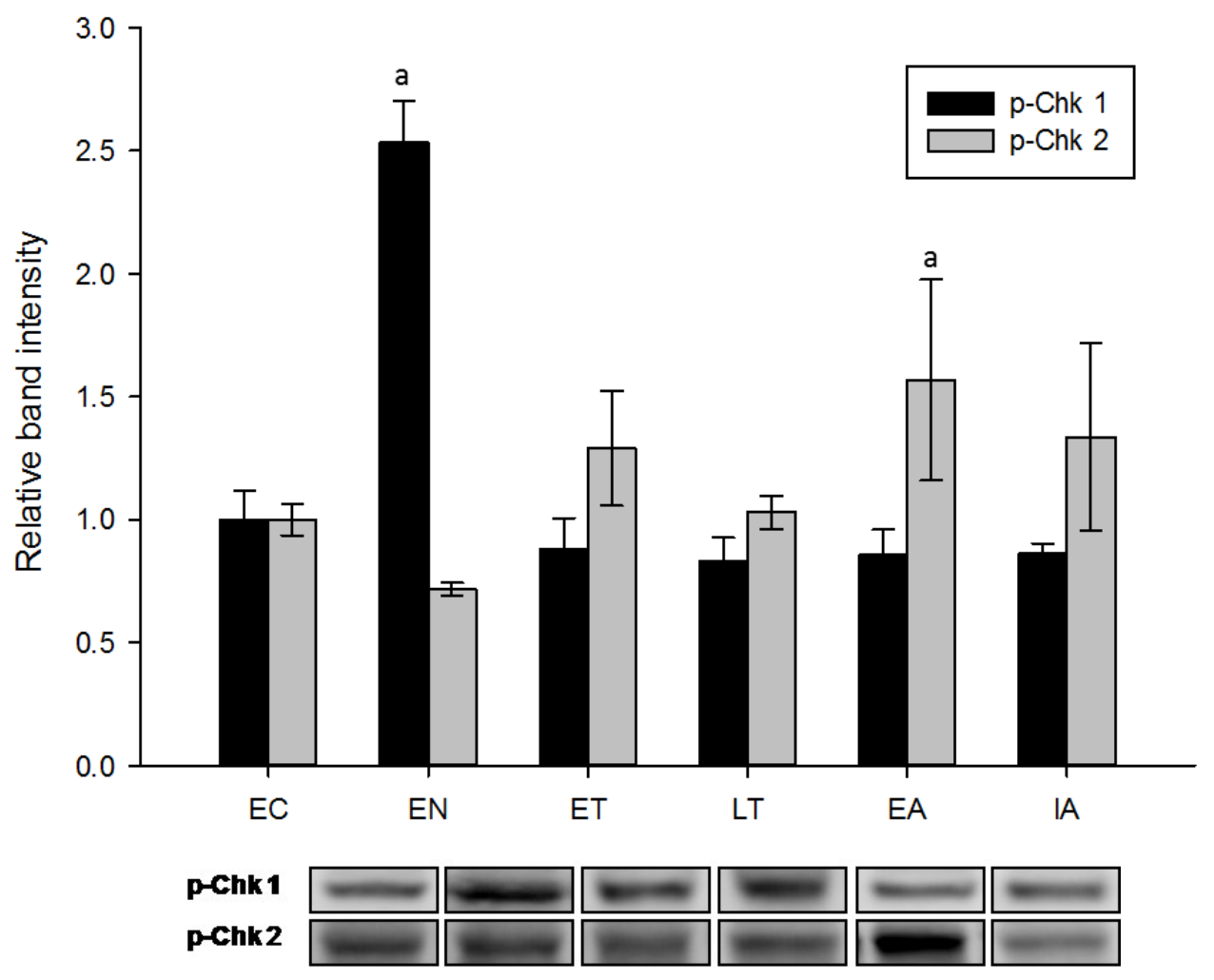

FIG. 6.8. Phosphorylation of checkpoint kinases in liver of I. tridecemlineatus as determined by Western immunoblotting.

Relative phosphorylation of p-Chk 1 (Ser-296) and p-Chk 2 (Thr-68) over the torpor-arousal cycle in liver of 13-lined ground squirrels. Western blots detected p-Chk 1 at $\sim 56 \mathrm{kDa}$, and p-Chk 2 at $\sim 62 \mathrm{kDa}$. Data are means \pm S.E.M., $\mathrm{n}=4-5$ independent trials on tissues from different animals. a-Significantly different from corresponding EC values $(\mathrm{P}<0.05$, ANOVA with post hoc Holm-Sidak test). 


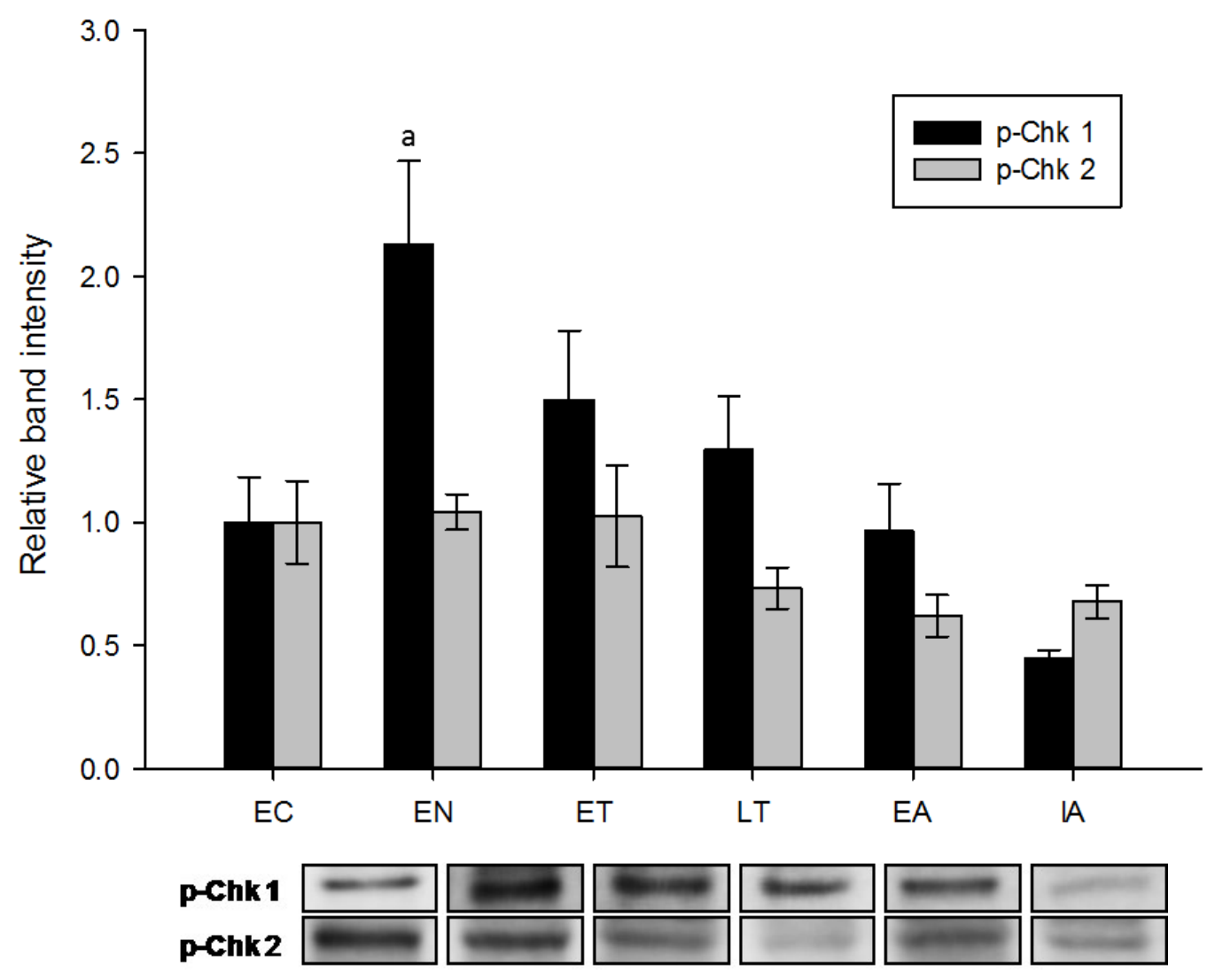

FIG. 6.9. Phosphorylation of checkpoint kinases in skeletal muscle of $I$. tridecemlineatus as determined by Western immunoblotting.

Relative phosphorylation of p-Chk 1 (Ser-296) and p-Chk 2 (Thr-68) over the torpor-arousal cycle in skeletal muscle of 13-lined ground squirrels. Representative Western blot bands of p-Chk 1 and p-Chk 2 are shown. Data are means \pm S.E.M., $n=4-5$ independent trials on tissues from different animals. a-Significantly different from corresponding EC values ( $\mathrm{P}<0.05$, ANOVA with post hoc Holm-Sidak test). 


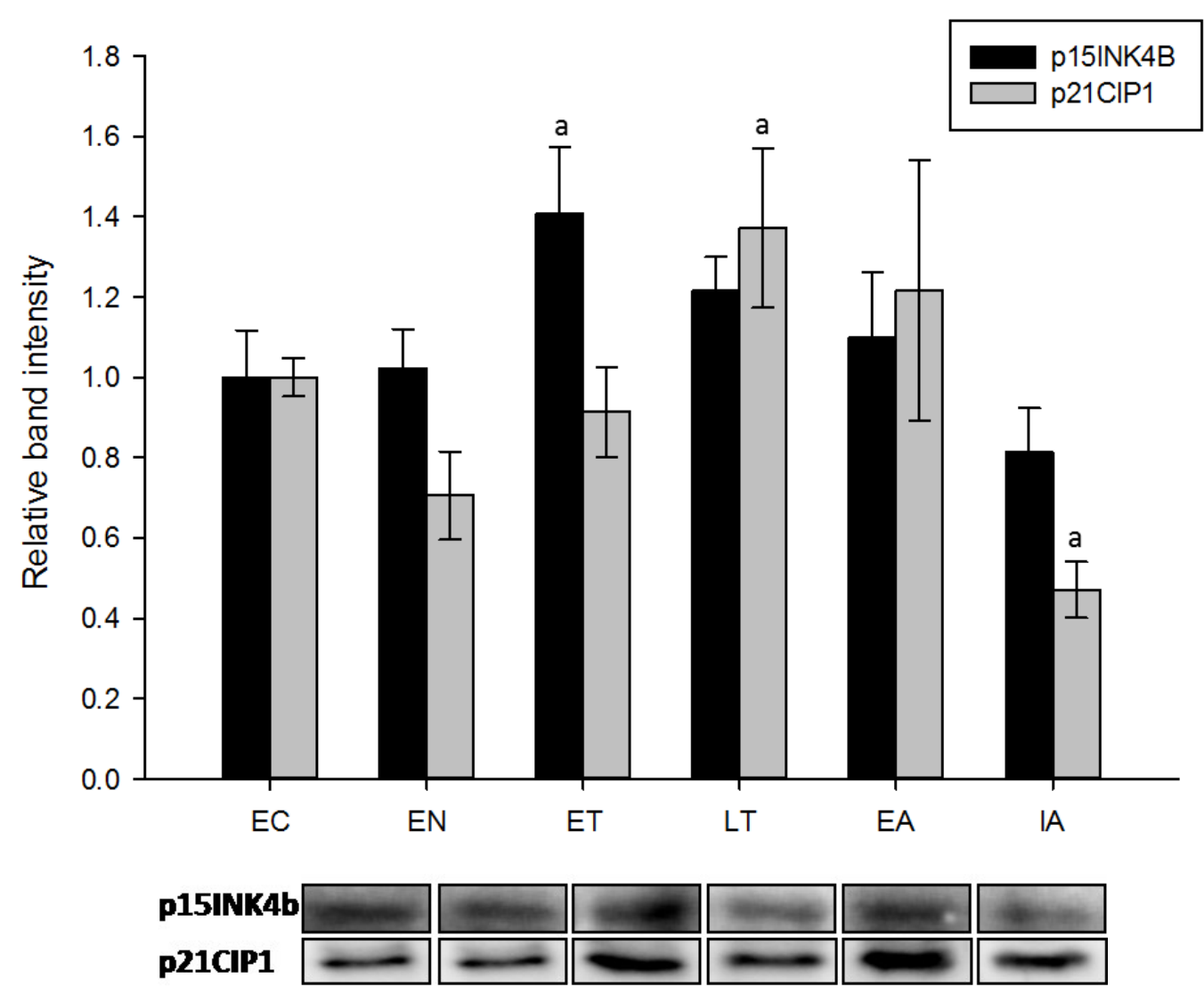

FIG. 6.10. Regulation pattern of cell cycle inhibitor proteins in liver of $I$. tridecemlineatus as determined by Western immunoblotting.

Relative expression of $\mathrm{p} 15 \mathrm{INK} 4 \mathrm{~b}$ and $\mathrm{p} 21 \mathrm{CIP} 1$ proteins over the torpor-arousal cycle in liver of 13-lined ground squirrels. Representative Western blot bands are shown and detected p15INK $4 \mathrm{~b}$ at $\sim 15 \mathrm{kDa}$ and $\mathrm{p} 21 \mathrm{CIP} 1$ at $\sim 21 \mathrm{kDa}$. Data are means \pm S.E.M., $\mathrm{n}$ $=4-5$ independent trials on tissues from different animals. a - Significantly different from corresponding EC values ( $\mathrm{P}<0.05$, ANOVA with post hoc Holm-Sidak test). 


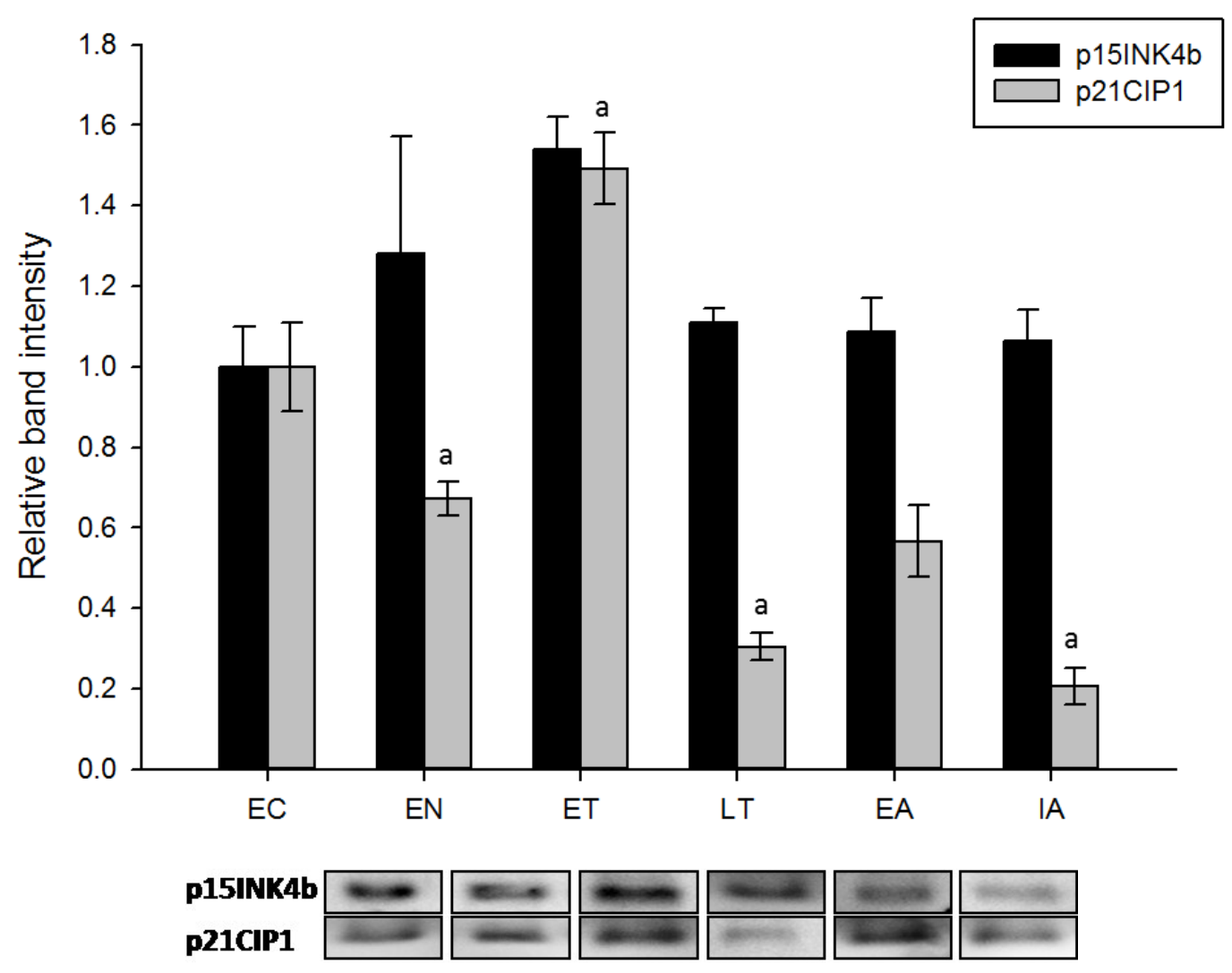

FIG. 6.11. Regulation pattern of cell cycle inhibitor proteins in skeletal muscle of $I$. tridecemlineatus as determined by Western immunoblotting.

Relative expressions of p15INK4b and p21CIP1 proteins over the torpor-arousal cycle in skeletal muscle of 13-lined ground squirrels. Representative Western blot bands of p15INK4b and p21CIP1 are shown. Data are means \pm S.E.M., $n=4-5$ independent trials on tissues from different animals. a-Significantly different from corresponding EC values $(\mathrm{P}<0.05$, ANOVA with post hoc Holm-Sidak test). 


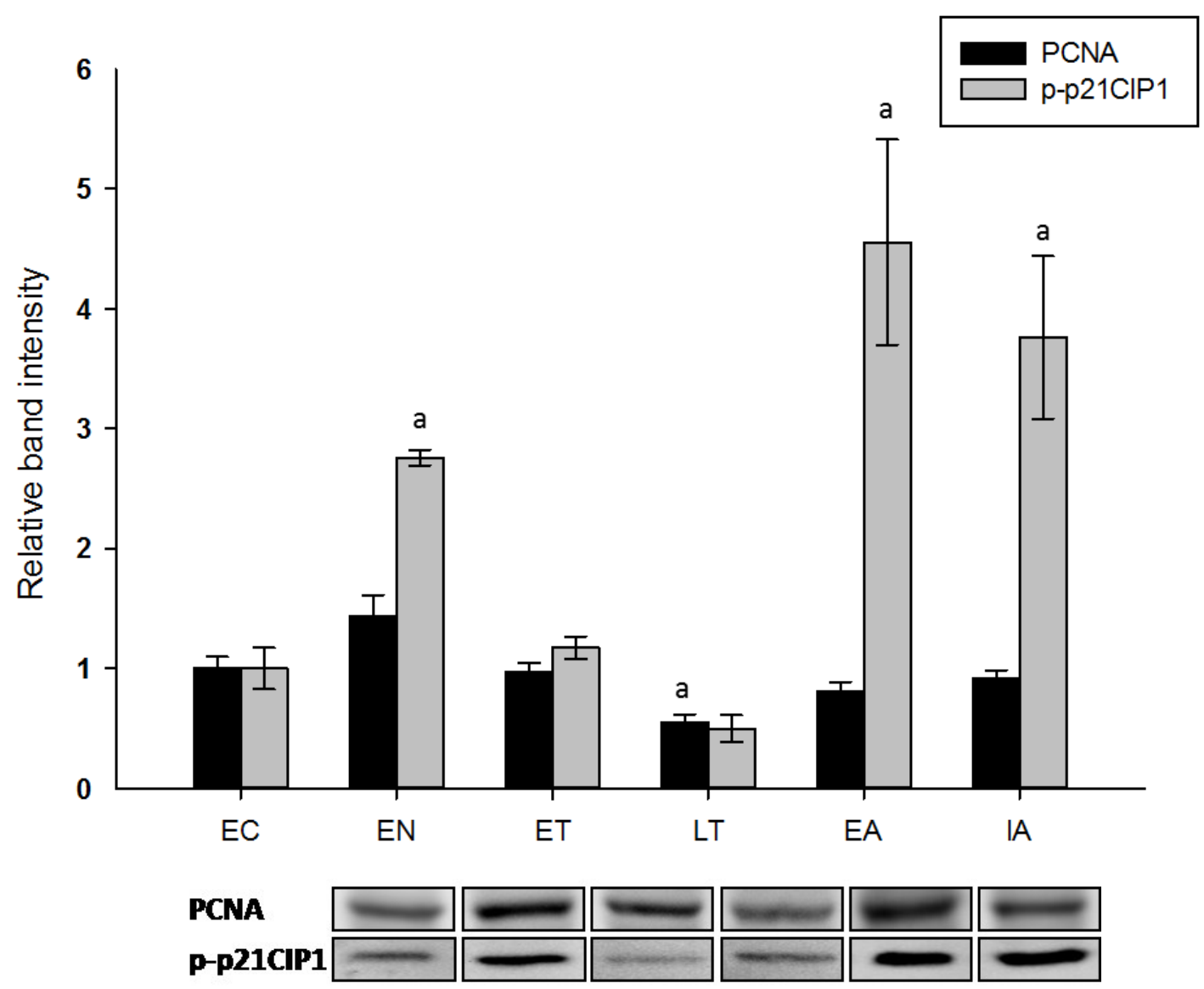

FIG. 6.12. Regulation pattern of PCNA protein and relative phosphorylation of p21CIP1 (Ser-146) in liver of I. tridecemlineatus as determined by Western immunoblotting.

Relative expression of PCNA protein and phosphorylation of p21CIP1 over the torpor-arousal cycle in liver of 13-lined ground squirrels. Western blot bands of PCNA were detected at $\sim 29 \mathrm{kDa}$, and p-p21CIP1 at $\sim 21 \mathrm{kDa}$. Data are means \pm S.E.M., $\mathrm{n}=4-5$ independent trials on tissues from different animals. a-Significantly different from corresponding EC values ( $\mathrm{P}<0.05$, ANOVA with post hoc Holm-Sidak test). 


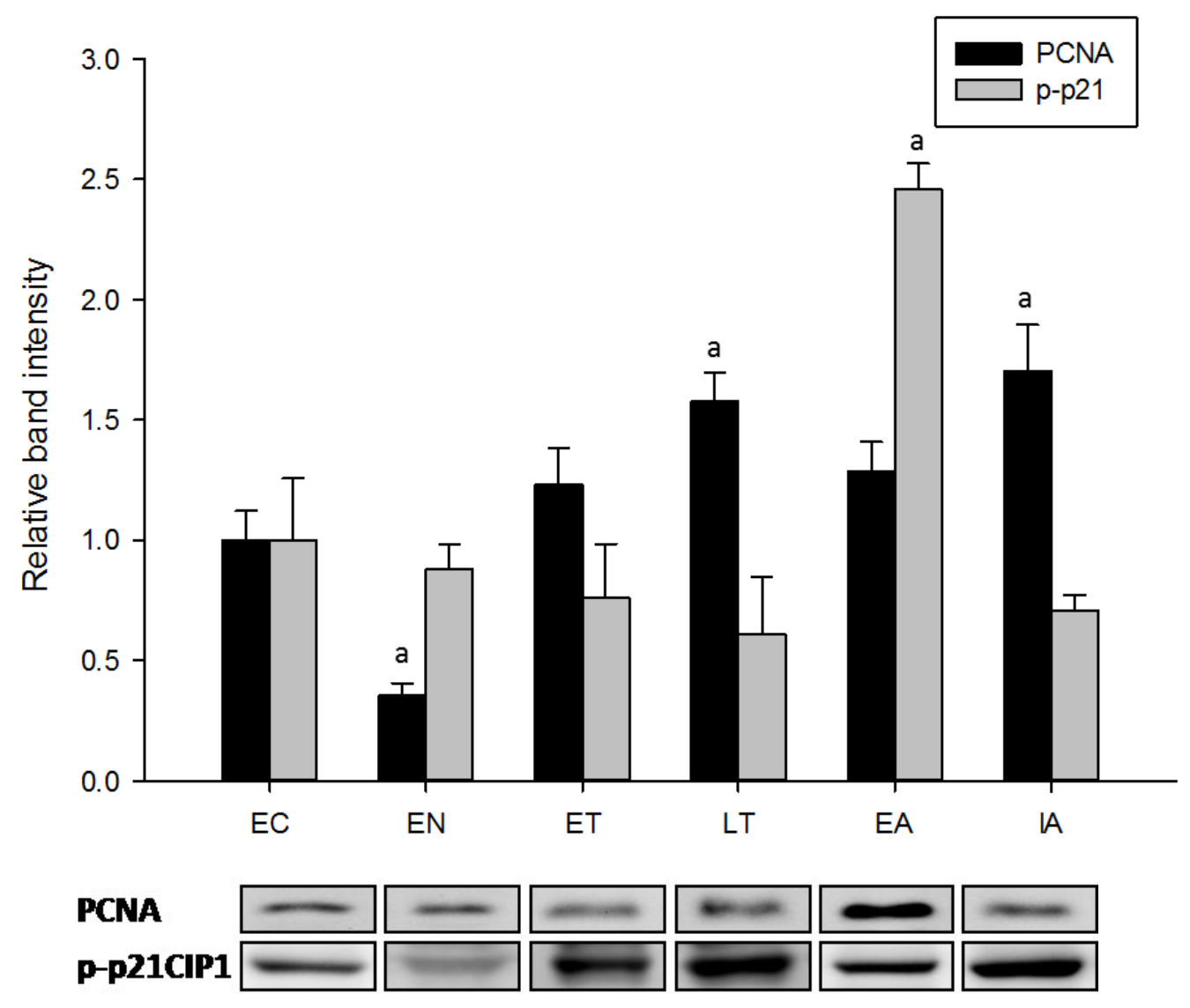

FIG. 6.13. Regulation pattern of PCNA protein and relative phosphorylation of p21CIP1 (Ser-146 ) in skeletal muscle of I. tridecemlineatus as determined by Western immunoblotting.

Relative expression of PCNA protein and phosphorylation of p21CIP1 protein over the torpor-arousal cycle in skeletal muscle of 13-lined ground squirrels.

Representative Western blot bands of PCNA and p-p21CIP1 are shown. Data are means \pm S.E.M., $\mathrm{n}=4-5$ independent trials on tissues from different animals. a-Significantly different from corresponding EC values $(\mathrm{P}<0.05$, ANOVA with post hoc Holm-Sidak test). 


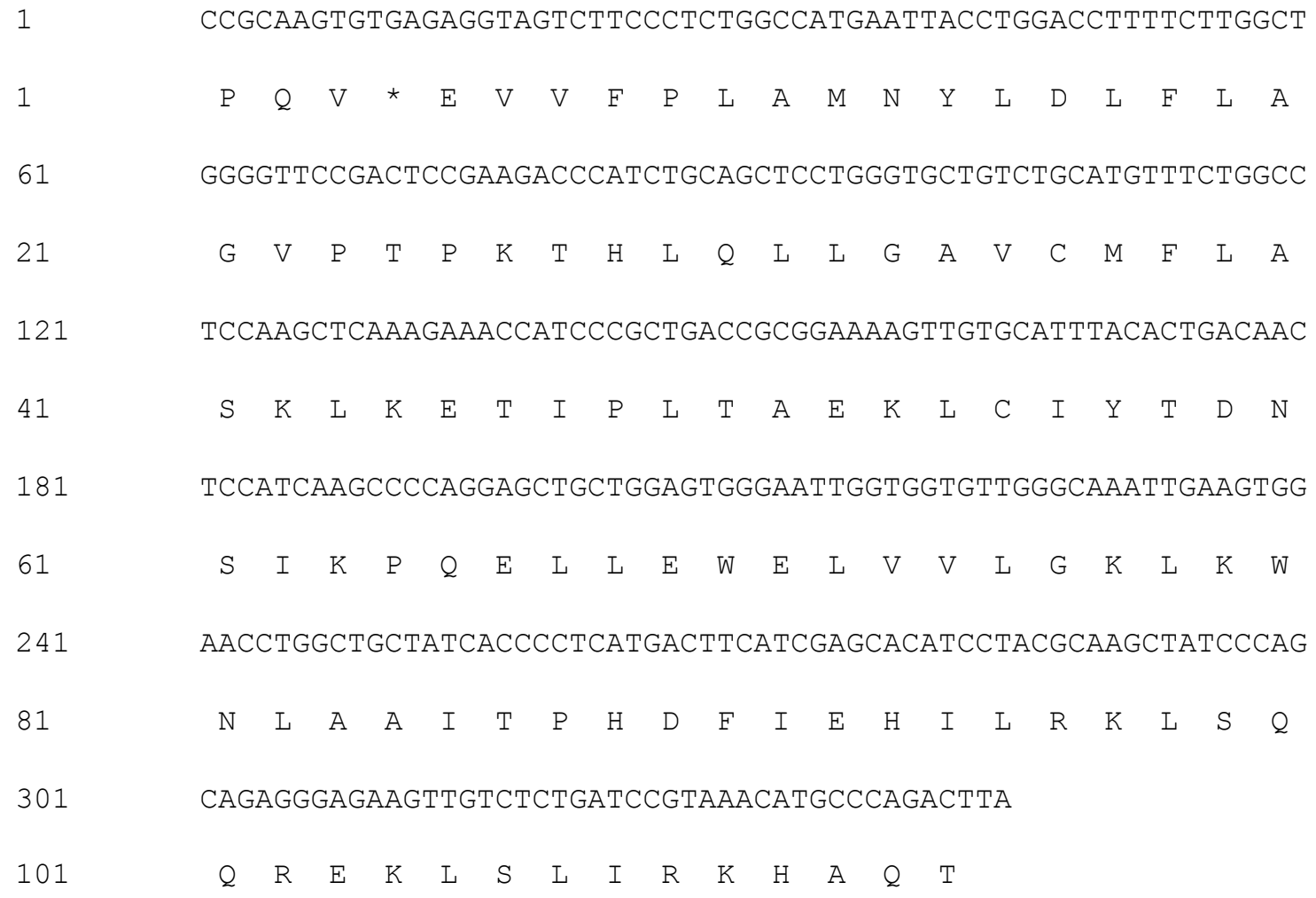

\section{FIG. 6.14. Partial cDNA sequence of cyclin D1}

Partial nucleotide sequence of cyclin DI amplified from muscle of $I$. tridecemlineatus and translated amino acid sequence. The open reading frame of the ground squirrel gene closely matched that of the human, mouse and rat genes. 
(A)
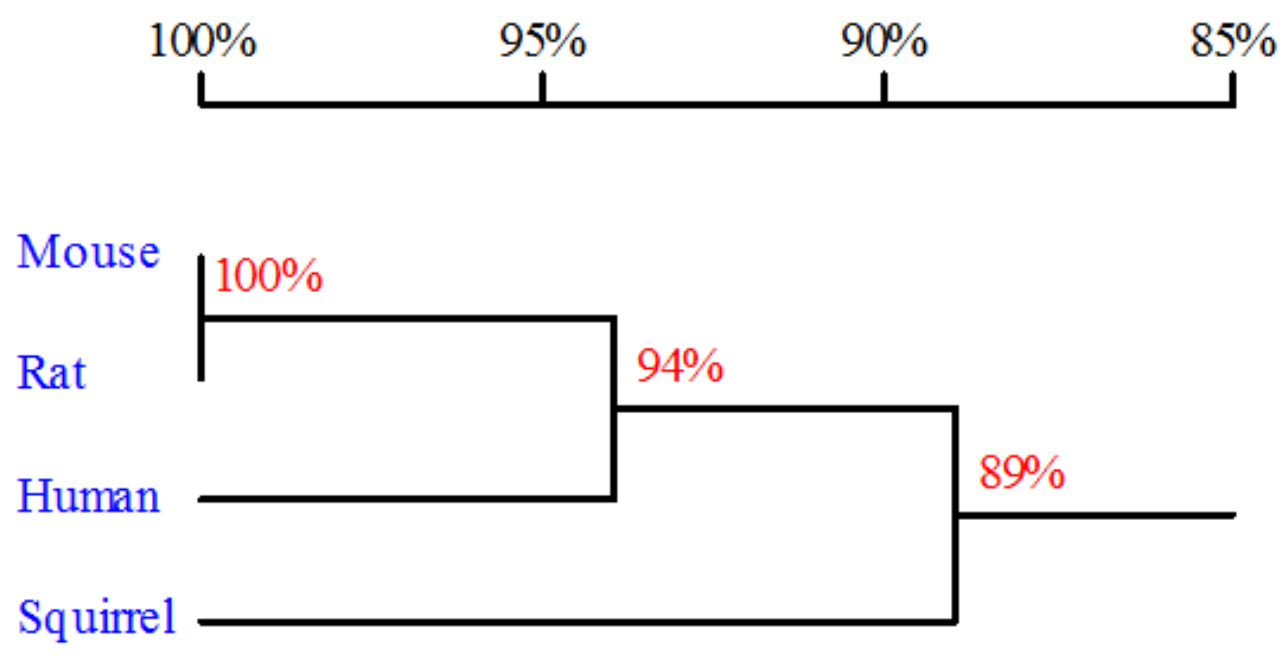

(B)

$$
\text { Mouse Rat Human Squirrel }
$$

$\begin{array}{lll}\text { Mouse } & 100 \% & \\ \text { Rat } & 100.0 \% 100 \% & \\ \text { Human } & 93.9 \% 93.9 \% 100 \% & \\ \text { Squirrel } & 89.2 \% 89.2 \% 89.6 \% 100 \%\end{array}$

\section{FIG 6.15. Cyclin D1 homology alignment.}

(A) Homology tree produced from alignment of I. tridecemlineatus cyclin D1 amino acid sequence with human (Homo sapiens), rat (Rattus norvegicus), and mouse (Mus musculus) sequences. The percentage values correspond to the degree of homology between species. (B) Homology matrix representing degree of conservation of $I$. tridecemlineatus cyclin D1 sequence compared with cyclin D1 from other mammals. 


\section{FIG. 6.16. Partial cDNA sequence of cyclin E1}

Partial nucleotide sequence of cyclin E1 amplified from muscle of $I$.

tridecemlineatus and translated amino acid sequence. The open reading frame of the ground squirrel gene closely matched that of the human, mouse and rat genes. 
(A)
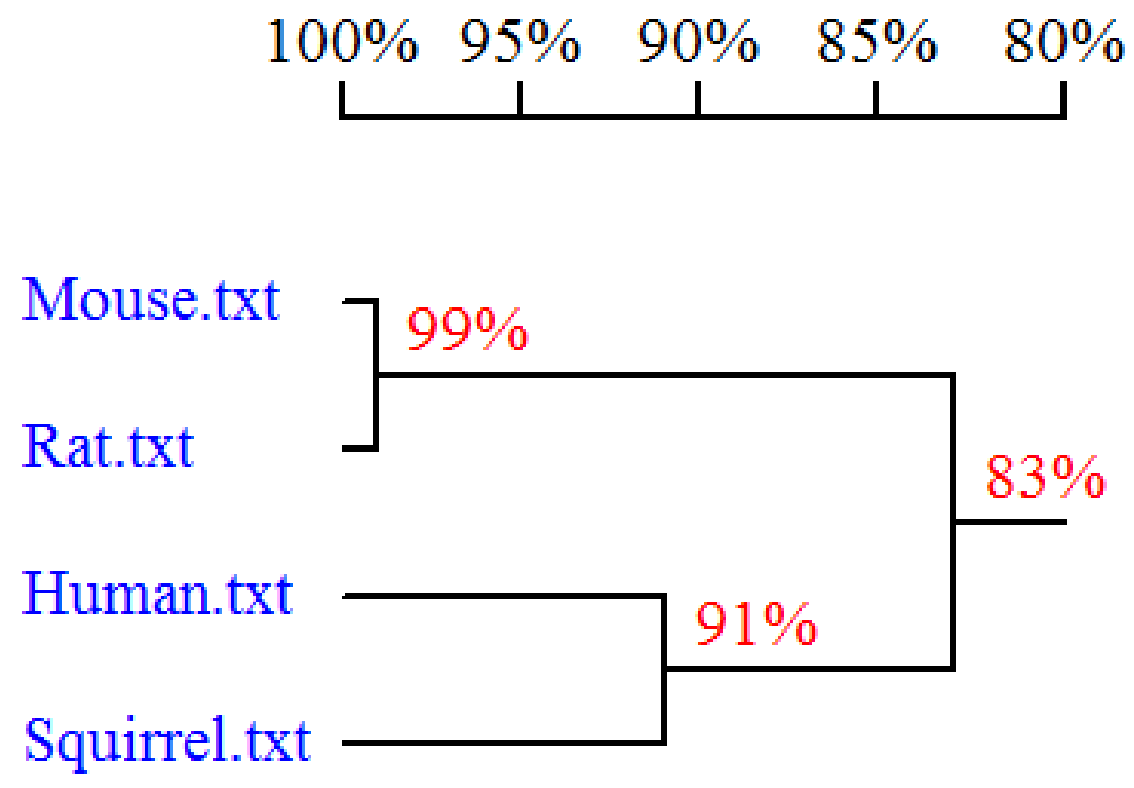

(B)

Mouse Rat Human Squirrel

Mouse

$100 \%$

Rat

$98.5 \% 100 \%$

Human

$79.4 \% 79.8 \% 100 \%$

Squirrel

$86.3 \% 86.3 \% 91.2 \% 100 \%$

\section{FIG 6.17. Cyclin E1 homology alignment.}

(A) Homology tree produced from alignment of I. tridecemlineatus cyclin E1 protein sequence with human (Homo sapiens), rat (Rattus norvegicus), and mouse (Mus musculus). The percentage values correspond to degree of homology between species. (B) Homology matrix representing degree of conservation of $I$. tridecemlineatus cyclin E1 sequence compared with PDK-1 from other mammals. 


\begin{tabular}{|c|c|c|c|c|c|c|c|c|c|c|c|c|c|c|c|c|c|c|c|c|}
\hline 1 & $A G 7$ & 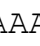 & 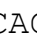 & $\mathrm{CC}$ & $\mathrm{GCA}$ & TC & 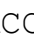 & $\mathrm{Tr}$ & $\mathrm{AT}$ & $\mathrm{GTG}$ & $\mathrm{AT}$ & $A A$ & $\mathrm{CZ}$ & $\mathrm{AC}$ & & & $\mathrm{ACT}$ & $A$ & $A G$ & GG \\
\hline 1 & $\mathrm{~S}$ & K & $Q$ & $\mathrm{P}$ & $\mathrm{A}$ & $\mathrm{F}$ & $\mathrm{T}$ & $I$ & $\mathrm{H}$ & $\mathrm{V}$ & D & $\mathrm{E}$ & A & $\mathrm{E}$ & $\mathrm{E}$ & $\mathrm{E}$ & $\mathrm{T}$ & Q & K & $\mathrm{R}$ \\
\hline 61 & $\mathrm{CCZ}$ & $\mathrm{CC}$ & $\mathrm{BA}$ & $A A I$ & $\mathrm{AGG}$ & $\mathrm{CZ}$ & $\mathrm{AC}$ & G & $\mathrm{BAC}$ & $A A T$ & $\mathrm{TC}$ & $\mathrm{TC}$ & $\mathrm{BC}$ & TI & $\mathrm{AGT}$ & $\mathrm{CZ}$ & $\mathrm{GCT}$ & $\mathrm{BCT}$ & $\mathrm{ACT}$ & $\mathrm{TA}$ \\
\hline 21 & P & $S$ & $E$ & $\mathrm{~K}$ & $\mathrm{R}$ & $\mathrm{T}$ & $\mathrm{E}$ & C & $\mathrm{E}$ & $\mathrm{N}$ & V & $\mathrm{L}$ & A & $\mathrm{F}$ & $S$ & $\mathrm{~T}$ & $A$ & A & $\mathrm{T}$ & $\mathrm{L}$ \\
\hline 121 & $\mathrm{CCZ}$ & $\mathrm{GG}$ & $\mathrm{CCZ}$ & $\mathrm{AGZ}$ & $A A I$ & $\mathrm{C}$ & $\mathrm{TC}$ & $\mathrm{TTC}$ & $\mathrm{CP}$ & $\mathrm{CTG}$ & $A 7$ & AT & $\mathrm{CCZ}$ & $\mathrm{TC}$ & $\mathrm{GAT}$ & $G I$ & $\mathrm{AGT}$ & ?T1 & $\mathrm{AAC}$ & $\mathrm{CA}$ \\
\hline 41 & $\mathrm{P}$ & G & $\mathrm{P}$ & $\mathrm{R}$ & $\mathrm{K}$ & P & $\mathrm{L}$ & V & P & $\mathrm{L}$ & D & $Y$ & P & $\mathrm{M}$ & $\mathrm{D}$ & G & $S$ & $\mathrm{~F}$ & $\mathrm{E}$ & $S$ \\
\hline 181 & $\mathrm{CC}$ & $\mathrm{CAC}$ & $\mathrm{AC}^{\prime}$ & $\mathrm{TC}$ & $\mathrm{GAT}$ & $\mathrm{TI}$ & $\mathrm{CZ}$ & $\mathrm{ATH}$ & $\mathrm{STC}$ & ГTA & $A z$ & $\mathrm{AT}$ & $\mathrm{BA}$ & $A C$ & $\mathrm{CAA}$ & $\mathrm{TC}$ & $\mathrm{AGT}$ & $\mathrm{ST}$ & $\mathrm{AT}$ & \\
\hline 61 & P & $\mathrm{H}$ & $\mathrm{T}$ & $\mathrm{M}$ & $\mathrm{D}$ & $I$ & S & $I$ & V & $\mathrm{L}$ & $\mathrm{E}$ & D & $\mathrm{E}$ & K & $\mathrm{P}$ & V & $S$ & $\mathrm{~V}$ & $\mathrm{~N}$ & $\mathrm{E}$ \\
\hline 241 & $\mathrm{GT}$ & $\mathrm{CCC}$ & GA & CAC & $\mathrm{CAC}$ & $A$ & $A$ & $A T H$ & $\mathrm{AC}$ & $\mathrm{ACA}$ & $\mathrm{AC}$ & $\mathrm{TT}$ & $\mathrm{AGC}$ & $A I$ & $\mathrm{ATG}$ & $A C$ & $\mathrm{GTC}$ & $A C$ & GI & $\mathrm{AG}$ \\
\hline 81 & V & $\mathrm{P}$ & D & $\mathrm{Y}$ & $\mathrm{H}$ & $\mathrm{E}$ & D & I & $\mathrm{H}$ & $\mathrm{T}$ & $Y$ & $\mathrm{~L}$ & $\mathrm{R}$ & $\mathrm{E}$ & $\mathrm{M}$ & $\mathrm{E}$ & $\mathrm{V}$ & K & C & K \\
\hline 301 & $\mathrm{CC}$ & I F & (T) & 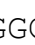 & TAC & 110 & 14 & & & & & & & & & & & & & \\
\hline 101 & P & $\mathrm{R}$ & V & G & $Y$ & $\mathrm{M}$ & 1 & & & & & & & & & & & & & \\
\hline
\end{tabular}

\section{FIG. 6.18. Partial cDNA sequence of $\operatorname{cyclin} A 2$}

Nucleotide sequence of cyclin A2 amplified from muscle of I. tridecemlineatus and translated amino acid sequence. The open reading frame of the ground squirrel gene closely matched that of the human, mouse and rat genes 
(A)
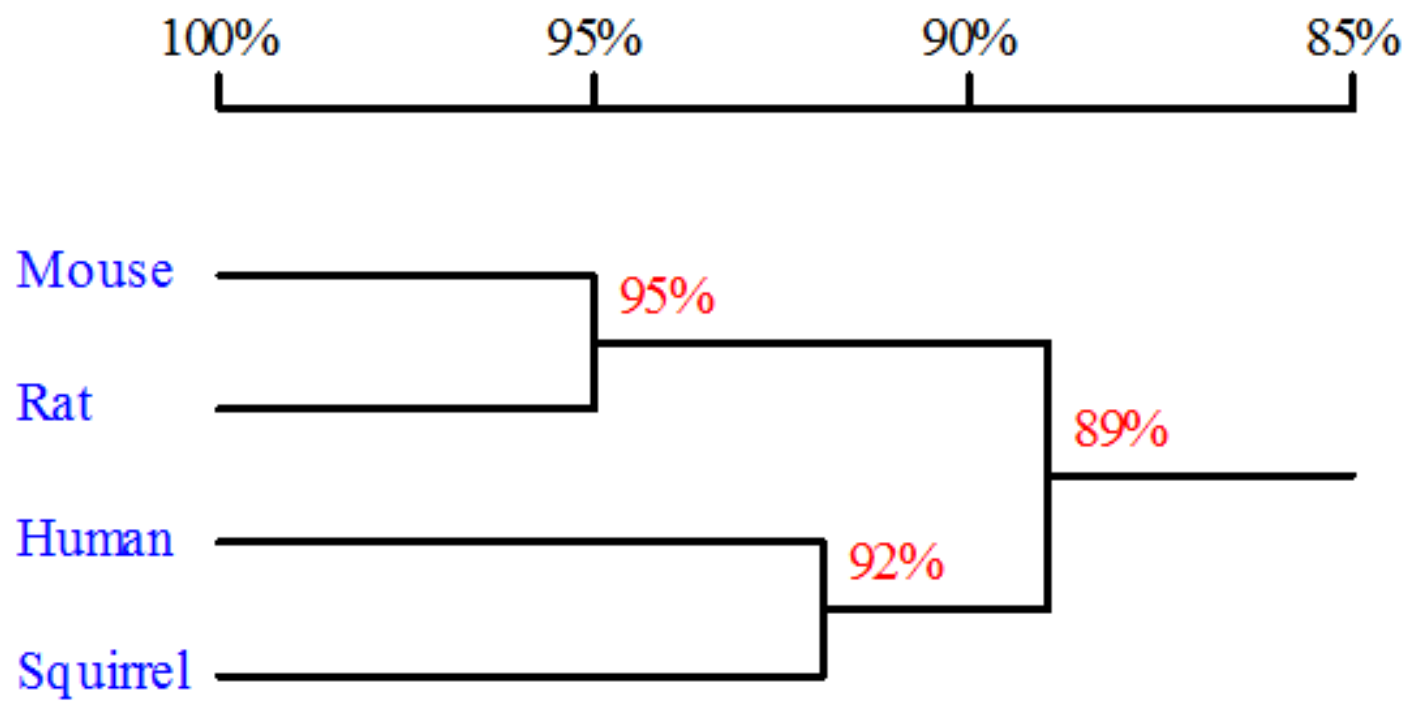

(B)

$$
\text { Mouse Rat Human Squirrel }
$$

Mouse

$100 \%$

Rat

$95.0 \% 100 \%$

Human

$88.1 \% 89.4 \% 100 \%$

Squirrel

\section{FIG 6.19. Cyclin A2 homology alignment.}

(A) Homology tree produced from alignment of I. tridecemlineatus cyclin A2 protein sequence with human (Homo sapiens), rat (Rattus norvegicus), and mouse (Mus musculus). The percentage values correspond to degree of homology between species. (B) Homology matrix representing degree of conservation of I. tridecemlineatus cyclin A2 sequence compared with PDK-1 from other mammals. 
CGGAAAgTATCTTCAGTATTGgTGACTTTGCTTTTGTGACTAACAACACTTACACTAATC

$\begin{array}{lllllllllllllllllll}E & S & I & F & S & I & G & D & F & A & F & V & T & N & N & T & Y & T & N\end{array}$ ACCAAATCAGACAGATGG

$\begin{array}{llllllllll}H & Q & I & \mathrm{R} & \mathrm{Q} & \mathrm{M}\end{array}$

\section{FIG. 6.20. Partial cDNA sequence of cyclin B1}

Nucleotide sequence of cyclin B1 amplified from muscle of I. tridecemlineatus and translated amino acid sequence. The open reading frame of the ground squirrel gene closely matched that of the human, mouse and rat genes. 
(A)

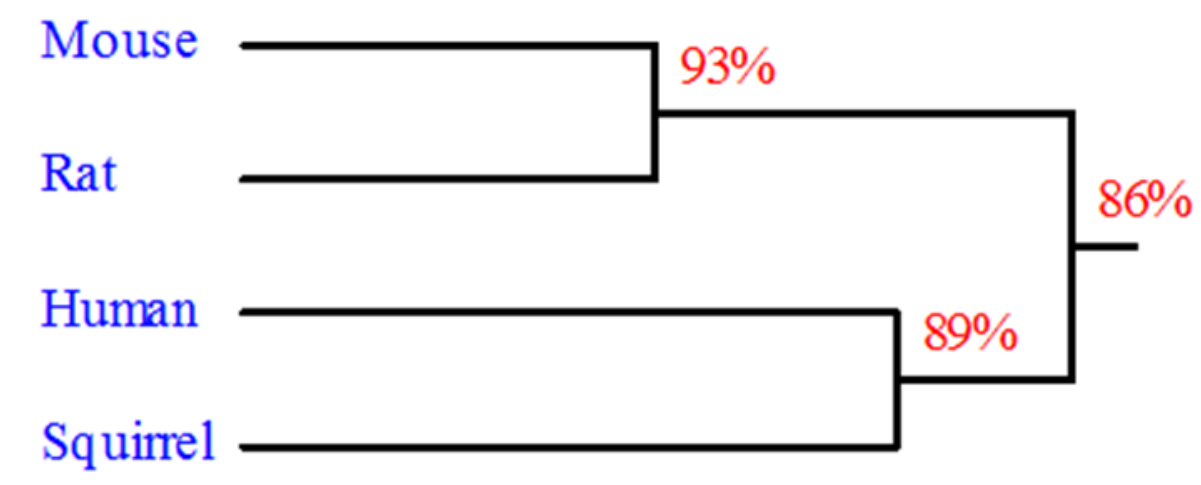

(B)

Mouse Rat Human Squirrel

Mouse

$100 \%$

Rat

$92.7 \div \quad 100 \%$

Human

$84.7 \% 84.4 \% 100 \%$

Squirrel

$87.4 \% 89.2 \% 89.0 \% 100 \%$

FIG 6.21. Cyclin B1 homology alignment.

(A) Homology tree produced from alignment of I. tridecemlineatus cyclin B1 protein sequence with human (Homo sapiens), rat (Rattus norvegicus), and mouse (Mus musculus). The percentage values correspond to degree of homology between species. (B) Homology matrix representing degree of conservation of I. tridecemlineatus cyclin B1 sequence compared with PDK-1 from other mammals. 
(A)
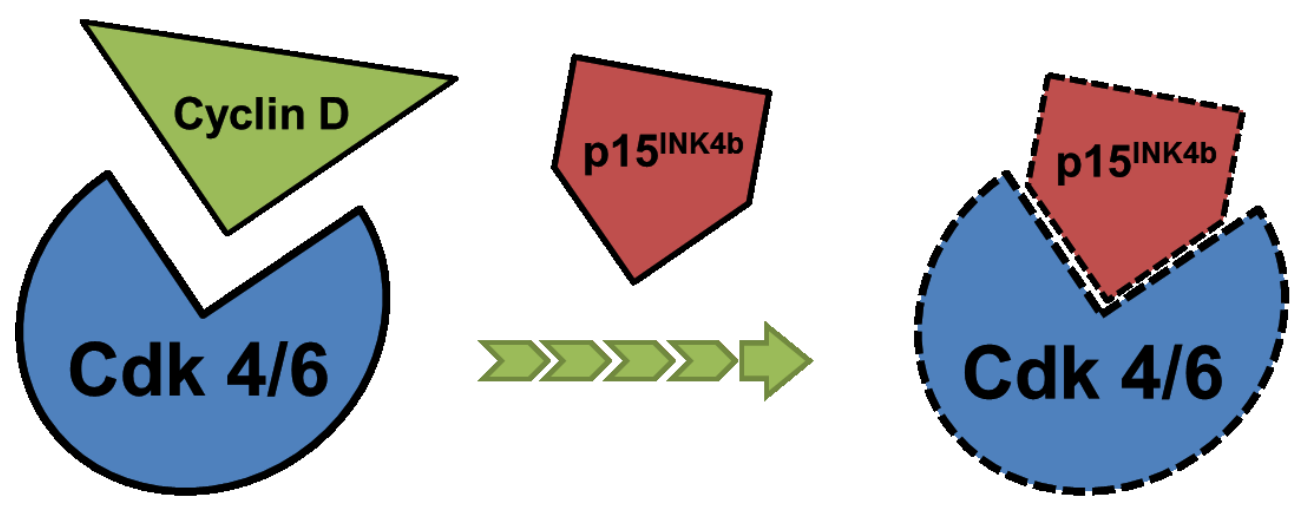

(B)
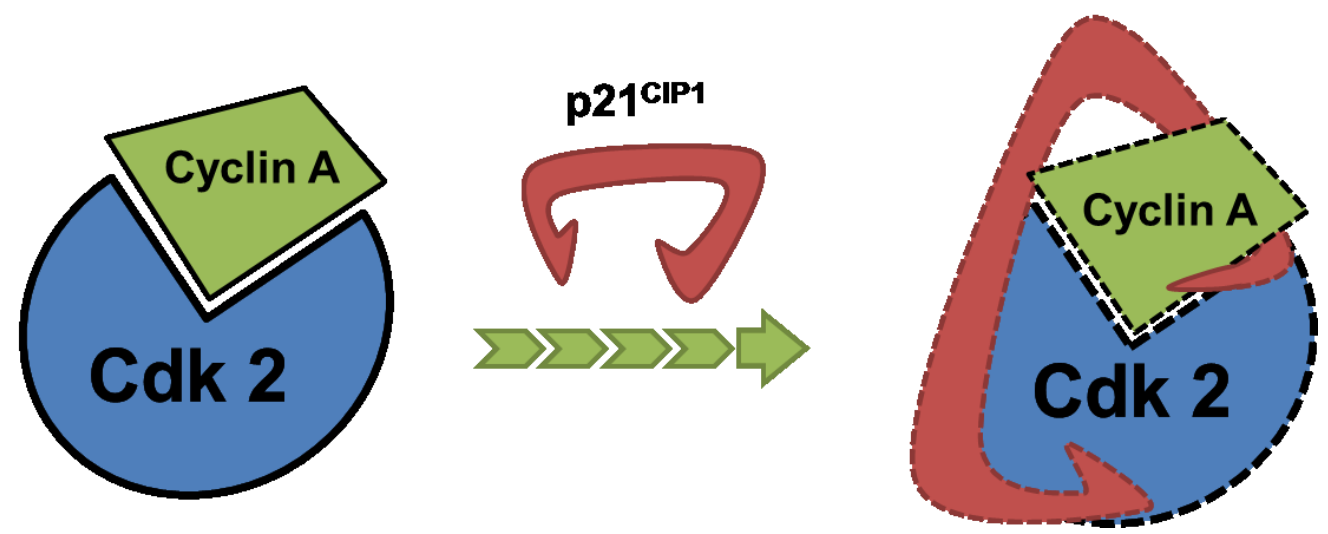

FIG. 6.22. Mechanism of CKI inhibition of Cdks. (A) p15INK4b competes for the Cdk $4 / 6$ binding site, inhibiting the binding of cyclin D. (B) p21CIP1 binds to the cyclin-Cdk protein complex, inhibiting both the regulatory and catalytic functions of the complex. 


\section{CHAPTER 7}

GENERAL DISCUSSION 


\subsection{GENERAL DISCUSSION}

In this thesis, the regulation patterns of major signaling pathways were presented, in relation to their involvement in energy metabolism. The role of pro-growth signaling pathways, mTOR signaling and the cell cycle were highlighted, along with the regulation of anti-proliferative Smad transcriptional signaling. These two types of signaling pathways (pro- and anti-growth) function antagonistically, altering metabolic regulation based on energy levels. Hibernation is a natural phenomenon, by which mammals undergo heterothermic metabolism by allowing their body temperatures to vary over a wide range, well below the threshold allowed in humans (although hibernators regulate $\mathrm{Tb}$ again if it falls to near $0^{\circ} \mathrm{C}$ ). It has been well documented that to achieve such phenotypic plasticity, a major rearrangement of energy metabolism must take place. The hibernation season consists of multiple cycles of torpor bouts and arousals that in ground squirrels are supported totally from catabolism of internal body fuel reserves stored before hibernation begins. Hence, animals must possess enough fuel storage to survive until environmental conditions are again favourable for active life. The switch towards a lipid-based metabolic fuel economy during the hibernation season is first achieved in late summer to early autumn, where ground squirrels nearly double their total body weight while tripling their fat mass (Klingenspor, 2003). This huge increase in white adipose tissue content is in the form of large amounts of triglycerides, which function as the primary source of metabolic fuel during hibernation (Dark, 2005). This includes a substantial increase in the proportion of total lipids that are polyunsaturated fatty acids (PUFA) which is necessary to maintain the fluidity of fat stores so that they can be mobilized when $\mathrm{Tb}$ falls to low values. To maximize energy availability to support vital 
functions through the hibernation season, overall energy expenditure is modulated via a reduction in non-essential cellular processes during torpor such as new protein synthesis and cell division. To undergo such a metabolic rearrangement, fined-tuned regulatory mechanisms must exist at the molecular level; these include mechanisms such as turning on/off transcription factors that control various downstream targets, or on-off switching of the activities of regulatory proteins via reversible protein phosphorylation.

While a number of studies have documented suppression of metabolic events (i.e. translational and transcriptional arrest, turning off ion pumps), little has been done to investigate the upstream molecular mechanisms that regulate these processes. The aim of my thesis was to investigate regulatory patterns of signaling pathways that have previously been shown to monitor cell growth and division, based on previous studies in other mammalian models. The thesis aimed to address two goals: 1 . Conservation of regulatory pathways in ground squirrels, and 2. Differential regulation of these pathways in energy conservation. To address these goals, I undertook a molecular approach, utilizing a variety of tools to characterize genomic and proteomic regulation of major signaling pathways, as well as assessing structure and function of key enzymes.

\subsubsection{Regulators of the translational complex; components of insulin signaling}

In Chapter 3, I looked at the regulation pattern of key components of the insulin signaling pathway during torpor, focusing on the mTORC1 complex due to its direct link towards regulating protein synthesis. Within the study, an emphasis was placed on the comparison of the regulation patterns between cardiac muscle and skeletal muscle. Phosphorylation of mTOR at its activating residue, Ser-2448, was found to be 
significantly reduced only in the skeletal muscle, but not the cardiac muscle during the torpid state. The reduction observed in the skeletal muscle was accompanied by an activation of mTORC1 inhibitors, via the dephosphorylation of TSC2 at its inhibitory residue (Thr-1462), and downregulation of the mTORC1 positive regulator, GßL, during torpor. Downstream of mTOR, consistent reductions in phosphorylation of 4E-BP1 and S6 ribosomal protein were also observed in skeletal muscle during torpor, but not in cardiac muscle. The regulation patterns of mTORC1 downstream in skeletal muscle suggested an overall suppressive state of the translational complex assembly, due to an increased activation of the cap-dependent protein synthesis inhibitor 4E-BP1, and a reduced activation of S6 protein in the 40S ribosomal complex. This pattern of regulation suggests a state of translational arrest in the skeletal muscle during torpor, but maintenance of protein translation in cardiac muscle.

The mTOR signaling pathway has previously been shown to play a major role in regulating muscle metabolism; since muscle cells in mature mammals do not actively divide, muscle masses are regulated by balances of anabolic and catabolic pathways (Stitt et al., 2004). The downregulation of mTOR has been associated with the onset of muscle atrophy, a pathology that has been previously documented in the skeletal muscle during torpor (Bodine et al., 2001; Wickler et al., 1991). Conversely, an upregulation of the mTOR pathway has been linked to the development of muscle hypertrophy (Bodine et al., 2001; Lee et al., 2007). Studies have previously documented a decrease in gastrocnemius mass of $21 \%$, and semitendinosus mass of $42 \%$ after periods of hibernation, likely a product of prolonged disuse of skeletal muscle during the hibernation season (Wickler et al., 1991). The reduction in mTOR signaling seen in my 
present studies would suggest that a suppression of active protein synthesis during torpor likely functions as a mechanism to reduce energy expenditure during torpor. However, this reduction in protein maintenance leads to the onset of muscle wasting, likely due to an imbalance of catabolic muscle metabolism, leading to the development of skeletal muscle atrophy as observed. Similar inhibition in mTOR signaling has also been observed in other metabolic stresses such as hypoxia; Brugarolas et al. showed that hypoxia-mediated mTOR suppression requires the functions of TSC2, to exert downstream effects on 4E-BP1 and S6 ribosomal protein (Brugarolas et al., 2006). In the cardiac muscle, however, mTOR signaling was maintained at a euthermic level, suggesting a basal rate of protein synthesis is maintained. This is remarkable considering the overall metabolic depression in torpor is $>95 \%$ of basal rate (Wang and Wolowyk, 1987). This evidence attests to the vital role of proper cardiac muscle function to provide continuous blood supply to organs during torpor. Although an upregulation of mTOR was not evident during ground squirrel torpor in my current studies, the development of ventricular hypertrophy by $21 \%$ in the cardiac muscle has been documented (Wickler et al., 1991). It is tempting to speculate that a basal rate of mTOR signaling might be viewed as an upregulation during torpor, due to the relative depression of all other metabolic processes during torpor. Although not confirmed, it could also be suggested that a decrease in rate of proteasome degradation occurs during torpor to shift the balance of cardiac muscle metabolism in favour of anabolic activities.

Although most significant changes in the regulation patterns of mTOR signaling were observed between euthermic control and late torpor stages, the two phases where the most drastic differences in metabolic functions are observed, the arousal phases in 
this study also showed significant alterations of mTOR signaling. In both skeletal and cardiac muscle, hyperphosphorylation of mTOR was observed during the early arousal phase. This suggests an increase in the capacity for protein synthesis as an aid to rapidly restoring euthermia and replenishing the active proteins that are required for proper metabolic functions during arousal. Indeed, proteins involved in antioxidant defence such as Nrf-2 have previously been shown to be upregulated during arousal, functioning to combat potential reperfusion damage associated with the rapid influx of oxygen and increase in aerobic metabolic rate (Morin et al., 2008). As the ground squirrel returns to a state of euthermia (during interbout arousal), essentially all components of mTOR signaling, upstream and downstream, returned to basal levels comparable to those previously observed during pre-torpor euthermia in both skeletal and cardiac muscle. This evidence suggests that a metabolic switch is in place, whereby induction of a phosphorylation cascade is aimed to turn mTOR signaling 'off' during torpor, while turning it back 'on' once euthermia is restored.

\subsubsection{Upstream of mTOR, role of PTEN in torpor}

The mTOR pathway is positioned downstream of the insulin signaling cascade, and is regulated by a number of kinases and phosphatases that are directly below the insulin receptor. The lipid derivative $\mathrm{PIP}_{3}$ molecule functions as a second messenger that relays the signals from the insulin receptor, which is regulated based on energy metabolism (Avruch, 1998). The $\mathrm{PIP}_{3}$ molecule functions to activate PDK-1, generating a phosphorylation cascade that leads to the activation of Akt kinase, along with its downstream targets (including key regulators of mTOR such as TSC2 and PRAS40) 
(Casamayor et al., 199 Manning and Cantley, 2009). The PTEN phosphatase displays intrinsic activity towards $\mathrm{PIP}_{3}$, catalyzing its dephosphorylation to $\mathrm{PIP}_{2}$, and thereby functioning as a negative regulator of Akt activation. In Chapter 4, enzymatic activities of PTEN along with transcriptional and translational regulation of PTEN and PDK-1 were characterized in the skeletal muscle during torpor. Skeletal muscle was chosen for this study due to the previous observations of a reduced mTOR activity state during torpor and this chapter explored the possibility of PTEN as a master switch to control mTOR signaling and protein synthesis in the skeletal muscle during torpor.

Previous studies have shown that insulin levels during the mid-hibernation season (December to March) are much the same as summer squirrels pre-hibernation (Buck et al., 2001). Hence, the reduction in insulin signaling, as evident by reduced Akt and mTOR phosphorylation could be a product of insulin resistance during torpor. Indeed, onset of insulin resistance has been documented in other hibernators such as the yellowbellied marmot during torpor (Florant et al., 1985). Previous studies have shown that increased PTEN expression is associated with increased insulin resistance in obese mice, therefore, it could also be suggested that PTEN functions can be related to the development of insulin resistance in hibernators (Lo et al., 2004). The findings of Chapter 4 showed that although PTEN transcription levels were unchanged over the torporarousal cycle, PTEN exhibited elevated levels of its unphosphorylated form during torpor. This posttranslational modification correlated with an increase in the catalytic activity of PTEN during torpor, with a 2.3-fold increase in $\mathrm{PIP}_{3}$ affinity. The regulatory mechanism of PTEN involves phosphorylation at three serine / threonine residues located in the C-terminal end of the protein. This regulatory phosphorylation has previously been 
demonstrated to affect PTEN activity as well as structural stability (Vazquez et al., 2001). As demonstrated in Chapter 4, the increased catalytic activity of PTEN that is regulated through dephosphorylation of residues at the C-terminal is associated with decreased structural stability, as previously described by Vazquez et al $(2000,2001)$. This suggests that the regulatory mechanism of PTEN activity is conserved between squirrels and humans, where phosphorylation induces a conformational change that reduces PTEN's interaction with target substrates, while masking the potential PEST sites for proteolytic cleavage.

The findings for ground squirrels suggest that PTEN is activated during the torpor stages of hibernation, and could function to inhibit the activities of PDK-1 and Akt through decreasing the level of intracellular PIP ${ }_{3}$. Indeed, phosphorylation of PDK-1 at its activation residue (Ser-241) was found to be significantly reduced during torpor. Although this study implicates PTEN as a major regulator that promotes insulin resistance during torpor, loss of function analysis would be needed to determine the exact role of PTEN in mTOR suppression during torpor. Overall, then, the findings of Chapter 3 and 4 provide evidence of reduced insulin signaling in ground squirrel skeletal muscle during torpor. This regulatory suppression involves the activation of PTEN phosphatase that could potentially function as an upstream master switch that 'turns off' Akt function during torpor. The reduction of mTOR signaling that is evident from the status of mTOR downstream targets (4E-BP, S6) suggests that assembly of the translational complex is stalled during torpor. Consequently, this reduction in insulin-mTOR signaling could result in the onset of muscular atrophy during torpor. Overall, this section of the thesis demonstrates a link between the molecular regulation of energy signaling events and the 
observed physiological changes in torpor.

\subsubsection{Activation of anti-proliferative factors in torpor}

The reduction in energy expenditure in torpor is accomplished by suppression of pro-growth and proliferative processes such as protein synthesis and gene transcription. These mechanisms usually involve turning off positive regulators of these pathways, frequently achieved via protein phosphorylation. For example, protein synthesis has been shown to be reduced via phosphorylation of eIF2- $\alpha$, while one mechanism for inhibiting gene transcription is via phosphorylation of RNA polymerase II (Frerich et al., 1998; Morin and Storey, 2008). However, another mechanism to induce arrest of cellular functions is by selective expression of inhibitory proteins or molecules. An example of such a mechanism is the activation of the Smad family of transcription factors, which induces the expression of a number of downstream targets that function as inhibitory proteins such as cell cycle inhibitors and microRNAs. Chapter 5 aimed to characterize the regulation pattern of Smad transcription factors, and their downstream targets in the torpor-arousal cycle. Two members of the Smad family of proteins were examined in liver and skeletal muscle using RT-PCR and Western blotting. Interestingly, although both Smad 2 and Smad 3 are thought to be activated by similar stimuli, only Smad 3 was found to be activated via phosphorylation. As well, tissue specific regulation was also observed, with increased phosphorylation of Smad 3 observed only in the liver, but not skeletal muscle. Accordingly, the activation of Smad 3 in liver was accompanied by an increase in its binding partner Smad 4, leading to an increase in nuclear inclusion for both proteins during torpor. Smad 3 DNA-binding activity was assessed in both liver and 
skeletal muscle, with liver showing a 1.5-fold increase in DNA binding while showing no change in muscle. The activation of Smad 3 during torpor was accompanied by a similar upregulation of Smad 3 downstream genes; transcript level of $p 15 I N K 4 b$ and $p 21 C I P 1$ were both found to be upregulated by $1.5-2$-fold during the torpor stages. As well, microRNAs that have previously been shown to be controlled by Smad proteins were also upregulated during torpor, with miR-21 and miR-29b overexpressed. The activation of Smad 3 is a primary response to binding of TGF- $\beta$ molecules at the receptor membrane (Massagué, 2005). However, previous studies have shown that levels of TGF$\beta$ are not significantly different throughout the torpor-arousal cycle in ground squirrels (Kutz and Carey, 2006). This could imply that activation of Smad 3 could be a product of stimulation by other cytokines such as activin or Nodal, both of which have been shown to induce Smad 3 phosphorylation (Massagué, 2005).

The increase in transcript levels of the cell cycle inhibitors $p 15 I N K 4 b$ and p21CIP1 suggests that the Smad signaling pathway can function to regulate cell division through activation of inhibitors. Both p15INK $4 \mathrm{~b}$ and $\mathrm{p} 21 \mathrm{CIP} 1$ have been shown to be associated with induction of cell cycle arrest and cellular senescence when overexpressed (Kim et al., 2006; Kagawa et al., 1999). The role of p15INK4b and p21CIP1 in cell division could potentially explain why activation of Smad transcription was only evident in the liver, but not skeletal muscle during torpor. It is likely that the terminally differentiated myocytes would not respond to increased expression of cell cycle inhibitors, as the tissues lacks proliferative capacity. Conversely, although liver hepatocytes do not undergo frequent cell division, they possess the capability to undergo cell cycle. Hence, the induction of $\mathrm{p} 15 \mathrm{INK} 4 \mathrm{~b}$ and $\mathrm{p} 21 \mathrm{CIP} 1$ via Smad 3 can function as a 
mechanism of stress response, by forcing the arrest of potential cell division that might take place over the hibernation season. Other than cell cycle inhibitors, Smad 3 is also involved in regulation of three different microRNAs that have previously been shown to be under the control of Smad (Kong et al., 2008). The upregulation of miR-29b is of special interest, due to its ability to target transcripts of $p 85 \alpha$, the catalytic subunit of PI3K. PI3-K functions as the antagonistic kinase to PTEN, that catalyses the phosphorylation of $\mathrm{PIP}_{2}$ to $\mathrm{PIP}_{3}$, activating PDK-1 kinase. Although not measured in this study, the increased level of miR-29b could suggest that PI3-K is selectively suppressed during torpor through microRNA interactions at the 3'UTR of the $p 85 \alpha$ transcript, a mechanism that has previously been shown to inhibit Akt signaling (Li et al., 2012). If so, Smad signal transduction would function to regulate multiple cellular processes, using different downstream effector molecules to promote its anti-proliferative signals on both the insulin signaling pathway and the cell cycle.

Although global suppression of metabolic processes is observed during hibernation, the activation of specific transcription factors is viewed as exceptions to the rule. Next to the observation of Smad activation, other stress-responsive transcription factors have also been shown to be activated during torpor; e.g. the hypoxia inducible factor $-1 \alpha(\mathrm{HIF}-1 \alpha)$ and nuclear factor $2(\mathrm{Nrf}-2)$ are both upregulated in multiple tissues during torpor (Morin and Storey, 2005; Morin and Storey, 2008). Elevated expression of Nrf-2 was associated with increased expression of its downstream target proteins such as $\mathrm{Cu} / \mathrm{Zn}$ superoxide dismutase, and heme oxygenase-1, proteins involved in the antioxidant defence system. 


\subsubsection{Cell cycle control}

Activation of the Smad downstream proteins $\mathrm{p} 15 \mathrm{INK} 4 \mathrm{~b}$ and $\mathrm{p} 21 \mathrm{CIP} 1$ was indicative of cell cycle arrest in liver during torpor. Although this was only evident in the liver, analysis of cell cycle components was also examined in skeletal muscle. The purpose of such a comparative analysis was to investigate the idea that components of cell division could function as housekeeping genes in non-proliferative tissues such as skeletal muscle, or if the components of the cell cycle have other biological functions outside of their canonical role. In Chapter 6, the key regulators of the cell cycle were profiled over the torpor-arousal cycle in both liver and skeletal muscle, using RT-PCR and Western blotting. In the liver, strong evidence of cell cycle arrest was present in the torpid stages of hibernation. A downregulation of cyclin D and cyclin E was observed, along with the elevation of $\mathrm{p} 15 \mathrm{INK} 4 \mathrm{~b}$ and $\mathrm{p} 21 \mathrm{CIP} 1$ proteins in early and late torpor stages of hibernation, respectively. The most interesting finding in the liver was the sequential upregulation of the cell cycle inhibitors. Activation of p15INK4b in the early torpor phase suggested inhibition of cell division at the $\mathrm{G}_{1}$ phase; this was followed by the activation of p21CIP1 in the late torpor phase, to inhibit cell division at the $\mathrm{G}_{1} \rightarrow \mathrm{S}$ phase. This type of sequential induction of cell cycle inhibitors suggests that cell cycle arrest is initiated during the early torpor phase, and reinforced in late torpor to ensure a complete stoppage before entering the S-phase, where the cell commits to division via DNA replication. This arrest of cell cycle progression appears to be reversed when the animal is aroused back to euthermia; increased expression of cyclin A and B at both the transcriptional and translational level indicated re-activation of the later stages of cell division (the $\mathrm{M}_{\text {and }} \mathrm{G}_{2}$ phases). This re-initiation of cell division can be viewed as a 
cardinal trait of cellular quiescence, by which a reversible growth / proliferation arrest is induced by a diverse number of anti-mitogenic signals (Coller et al., 2006). Therefore, it is likely that the state of cellular quiescence observed in liver during torpor is induced by the anti-mitogenic effects of TGF- $\beta$ signaling pathways, through which the Smad family of transcription factors are activated by a number of anti-mitogenic factors (Ten Dijke, 2002).

In skeletal muscle, the majority of components involved in cell cycle regulation were observed to be stable throughout the torpor-arousal cycle. Transcript levels of cyclins, along with protein levels of $\mathrm{p} 15 \mathrm{INK} 4 \mathrm{~b}$ and most of the four Cdks were unchanged over the stages of torpor-arousal. However, cyclin proteins underwent differential expression throughout the torpor-arousal cycle, with activation / repression patterns that were distinct from those observed in liver. Since the induction of cell cycle arrest is likely to be accomplished via a combination of different cellular mechanisms (e.g. reduced cyclin and Cdk levels, increased cell cycle inhibitors), changes in cyclin levels alone were not likely to induce a cellular change. Therefore, this suggests that cyclin proteins may possess alternative functions in non-proliferative tissues, one that is outside of the canonical role of cell cycle regulation. Indeed, the downregulation of cyclin D is likely to be involved in muscle metabolism, since it has previously been shown that cyclin D act as an inhibitor of MEF-2 function. MEF-2 is a transcription factor that control structural and contractile proteins in the skeletal muscle, and has been shown to be activated during ground squirrel torpor, which has been implicated in the maintenance of muscle myogenesis and structural retention. (Larzaro et al., 2002; Tessier and Storey, 2012). 
The process of cell division is highly energy expensive, involving the replication of DNA, the additional synthesis of many macromolecular components, and the division process itself. In animals that undergo a drastic reduction in energy metabolism to enter a hypometabolic state in response to environmental cues, it should not be a surprise that a similar rearrangement of cell cycle components also takes place at the molecular level. It has been well documented that arrest of cell division is a component of the stress response and of hypometabolism, as observed in both unicellular and multicellular organisms, in response to a range of stressful signals including nutrient deprivation, reduced oxygen availability, and many others (Storey, 2012). For example, nitrogen deprivation has been shown to induce $\mathrm{G}_{1}$ cell cycle arrest in yeast, whereas cell cycle arrest in multicellular organisms is known to occur in response to nutrient deprivation in C. elegans, hypoxia in Drosophila melanogaster, and anoxia in turtles (Gallego et al., 19976; Fukuyama et al., 2006; Douglas et al., 2005 Biggar and Storey, 2012). It is plausible to now consider cell cycle arrest as one of the primary responses during metabolic reprogramming, although many questions still remain to be answered before we will fully understand the common versus disparate regulatory principles that control the arrest of cell growth and proliferation in response to different forms of environmental stress.

\subsubsection{Outlooks}

This thesis aimed to characterize the regulatory processes of energy related metabolic functions in response to hibernation in ground squirrels. The components of the insulin signaling pathways were found to be differentially regulated between cardiac 
and skeletal muscle. Similarly, anti-mitogenic signalling by Smad displayed tissue specific modes of regulation, functioning to induce cell cycle arrest only in the liver, but not skeletal muscle during torpor. The dependence of complex cellular pathways on energy levels as shown in previous models such as tumour progression in malignancies (state of high energy consumption), and C. elegans metabolism during dauer stage (state of diapause) led me to investigate the importance of energy status and metabolic functions. This relationship is a fundamental principle of how animals adapt to various different types of environments via reprogramming of their metabolism, but also acts as the fundamental problem in many metabolic disorders, such as type-2 diabetes. Although basal metabolic rate may be depressed by $>95 \%$ during hibernation, this thesis shows examples of both cellular processes that are suppressed during torpor, and processes that are activated during torpor. These results further reinforce the idea that a hypometabolic state should be considered an actively regulated process, whereby coordinated controls on metabolism result in a state of dormancy. The most remarkable trait, however, is the reversibility of these metabolic controls when animals arouse back to euthermia, where previous evidence of suppression or activation in torpor is brought back to basal euthermic levels, as seen in the regulation of mTOR, Smad signaling, and the cell cycle. Although much evidence has been accumulated to date on many regulatory mechanisms involved in hibernation, biological cues that induce entry into hibernation are still largely unknown. Can active hormonal regulation induce entry into hibernation absent of environmental cues? What are the regulatory mechanisms that alter the internal body temperature set points in the hypothalamus? These are just examples of questions that still need to be answered in hibernation research, if we seek to fully understand, and 
appreciate this incredible natural phenotypic plasticity that is expressed in many species of mammals. 


\section{APPENDICES}




\section{Appendix A: List of Publications}

\section{Published / In Press}

*Equal contribution of authors

1. Biggar* KB, Wu CW*, and Storey KB. (2014) High-throughput amplification of mature microRNA in uncharacterized animal models using polyadenylated RNA and stem-loop RT-PCR. Anal Biochem (In Press - YABIO-S-14-00298).

2. Wu CW and Storey KB (2014). FoxO3a-mediated activation of stress responsive genes during early torpor in a mammalian hibernator. Mol Cell Biochem. 390(1-2): 185-195.

3. Tessier SN, Audas TE, Wu CW, Lee S, Storey KB (2014). The involvement of mRNA processing factors TIA-1, TIAR, and PABP-1 during mammalian hibernation. Cell Stress Chaperones (In Press; DOI - 10.1007/s12192-014-05058).

4. Wu CW, Biggar KK, and Storey KB. (2013) Dehydration mediated microRNA response in the African clawed frog Xenopus laevis. Gene. 592(2): 269 - 275.

5. Wu CW, Reardon AJ, and Storey KB. (2013) Effects of hibernation on regulation of mammalian protein phosphatase type-2-A. Cryobiology. 3(66): $267-274$.

6. Storey KB and Wu CW. (2013) Stress response and adaptation: a new molecular toolkit for the 21st century. Comp Biochem Physiol A Mol Integr Physiol. 165(4): $417-428$.

7. Wu CW, Biggar KK, and Storey KB. (2013) Biochemical adaptations of mammalian hibernation: exploring squirrels as a perspective model for naturally induced reversible insulin resistance. Braz J Med Biol Res. 46(1): 1 - 13.

8. Wu CW and Storey KB. (2012) Regulation of the mTOR signaling network in hibernating thirteen-lined ground squirrels. J Exp Biol. 215(Pt 10): 1720 -1727.

9. Wu CW and Storey KB. (2012) Pattern of cellular quiescence over the hibernation cycle in liver of thirteen-lined ground squirrels. Cell Cycle. 11(9): $1714-1726$.

\section{In Preparation (Completed Projects)}

10. Biggar KK*, Wu CW* Tessier SN, Zhang J, Storey KB. MAPK signaling in primate torpor, evidence of metabolic suppression. (In preparation)

11. Tessier SN, Zhang J, Biggar KK, Wu CW, Pifferi F, Perret M, Storey KB. The regulation of the $\mathrm{PI} 3 \mathrm{~K} / \mathrm{AKT}$ pathway during primate torpor in the grey mouse 
lemur, Microcebus murinus. (In preparation)

12. Wu CW, Bell R, Storey KB. Regulation of PTEN function and structural stability in hibernating thirteen-lined ground squirrels (In preparation)

13. Wu CW, Storey KB. Activation of SMAD 3 mediated transcriptional response in hibernating thirteen-lined ground squirrels (In preparation)

14. Wu CW*, Biggar KK*, Tessier SN, Zhang J, and Storey KB. Modulation of gene expression in key survival pathways during periods of torpour in the grey mouse lemur, Microcebus murinus. (In preparation)

15. Biggar KK, Wu CW, Pifferi F, Storey KB. MicroRNA identification and expression in the grey mouse lemur, Microcebus murinus, a primate capable of hibernation. (In preparation)

16. Wu CW, Biggar KK, Storey KB. Regulation of adipogenesis related microRNAs in ground squirrel torpor, comparative analysis of white vs. brown adipose tissue. (In preparation).

17. Tessier SN, Zhang J, Biggar KK, Wu CW, Pifferi F, Perret M, Storey KB. Molecular controls of fuel utilization during primate torpour in the grey mouse lemur, Microcebusm murinus.

18. Tessier SN, Zhang J, Biggar KK, Wu CW, Pifferi F, Perret M, Storey KB. The regulation of the PI3K/AKT pathway during primate torpour in the grey mouse lemur, Microcebus murinus.

19. Zhang J, Tessier SN, Biggar KK, Wu CW, Pifferi F, Perret M, Storey KB.Transcriptional and post-transcriptional control in the torpid grey mouse lemur, Microcebus murinus.

20. Biggar KK, Wu CW, Storey KB. MicroRNA profiling in brain of anoxic tolerant Trachemys scripta elegans. (In preparation)

21. Wu CW, Biggar KK, Mathialagan M, Storey KB. Freezing mediated ChREBP transcriptional response in wood frog $R$. sylvatica. (In preparation)

22. Wu CW, Biggar KK, Storey KB. Differential distribution of translated genes in polysome profiles in ground squirrels during hibernation. (In preparation)

23. Wu CW, Storey KB. Mechanism of retinoblastoma-E2F transcription regulation of cell cycle in mammalian torpor. (In preparation) 
24. Wu CW, Biggar KK, Tessier SN, Zhang J, Storey KB.Activation of antioxidant and heat shock protein responses during periods of primate torpour in the mouse lemur, Microcebus murinus. 


\section{Appendix B: Communication at Scientific meetings}

\section{Poster Presentations:}

Wu CW, and Storey KB. Roles of the mTOR signaling pathway in hibernating ground squirrels, a differential suppression of active protein synthesis. ACCryo 2013, Miami, Florida, USA. January 2-7, 2013.

Wu CW, Bell R, and Storey KB. Regulation of PTEN function and structural stability in hibernating thirteen-lined ground squirrels. ACCryo 2013, Miami, Florida, USA. January 2-7, 2013.

Wu CW, Bell R, and Storey KB. Regulation of PTEN function and structural stability in hibernating thirteen-lined ground squirrels. 19th Methods in Protein Structure Analysis symposium, Ottawa, Canada, June 25-28. 2012.

Wu CW, and Storey, KB. Roles of the mTOR signaling pathway in hibernating ground squirrels, a differential suppression of active protein synthesis. Society for Cryobiology, 49th Annual Meeting, Rosario, Argentina, June 3-6, 2012.

Wu CW, and Storey, KB. Roles of the mTOR signaling pathway in hibernating ground squirrels, a differential suppression of active protein synthesis. $5^{\text {th }}$ annual meeting, Canadian Society for Life Science Research. Montreal, Canada, August 13-14, 2010.

Wu CW, and Storey, KB. Characterization of type-2 protein phosphatase PP2A and PP2C in skeletal muscle of hibernating Richardson's ground squirrel, Spermophilus richardsonii. $7^{\text {th }}$ annual Ottawa-Carleton Institute of Biology Symposium. Ottawa, Canada, April 13-14, 2010.

Wu CW, and Storey, KB. Characterization of type-2 protein phosphatase PP2A and PP2C in skeletal muscle of hibernating Richardson's ground squirrel, Spermophilus richardsonii. $12^{\text {th }}$ annual Chemistry \& Biochemistry Graduate Research Conference. Montreal, Canada, November 20-21, 2009.

\section{Oral Presentations:}

Wu CW. Molecular adaptations of mammalian hibernation; links between metabolic depression and aging. 2014 American Aging Association Annual Meeting. San Antonio, Texas, May 31 - June 2, 2014. 
Wu CW, Storey KB. Molecular adaptations of mammalian hibernation; regulation of energy dependent cellular processes during metabolic depression. TM's 3rd World Molecular \& Cell Biology Online Conference. Feb, 2014.

Wu CW. Hypometabolism in mammals: role of translational regulation in torpor survival. $50^{\text {th }}$ annual meeting, Canadian Society of Zoologists. Ottawa, ON, May 16 $-20,2011$.

\section{Invited Lectures}

Wu CW. Biochemical Adaptation in Mammalian Hibernation - Regulation of Insulin / Akt Signaling Pathway. Invited departmental lecture. University of Florida, department of biology. Mar. 13, 2014.

Wu CW. Molecular mechanisms of mammalian hibernation; links between metabolic depression and aging. Invited departmental lecture. Harvard Medical School, division of genetics. Dec. $6^{\text {th }}, 2013$.

Wu CW. Molecular regulation of insulin signaling pathway and protein synthesis during hibernation Invited departmental lecture. University of Minnesota. Nov. $21^{\text {st }}$, 2013.

Wu CW. Biochemical adaptation of mammalian hibernation, regulation of energetic signaling pathways. Invited departmental lecture. Buck Institute for Research on Aging. April 8 ${ }^{\text {th }}, 2013$. 


\section{Appendix C: PCR amplification cycles}

\begin{tabular}{|c|c|c|}
\hline Gene & $\begin{array}{c}\text { PCR Cycle: } \\
\text { Liver }\end{array}$ & $\begin{array}{c}\text { PCR Cycle: } \\
\text { Muscle }\end{array}$ \\
\hline \multicolumn{3}{|l|}{ Chapter 4} \\
\hline PTEN & - & 25 \\
\hline$P D K-1$ & - & 26 \\
\hline \multicolumn{3}{|l|}{ Chapter 5} \\
\hline SMAD2 & 38 & 38 \\
\hline SMAD3 & 37 & 37 \\
\hline SMAD4 & 32 & 33 \\
\hline$\alpha$-tubulin & 25 & 25 \\
\hline Actin- $\beta$ & 30 & 32 \\
\hline microRNA universal primer & - & - \\
\hline$m i R-29 b$ & 28 & 25 \\
\hline$m i R-21$ & 26 & 25 \\
\hline $\operatorname{miR} 155$ & 28 & 31 \\
\hline \multicolumn{3}{|l|}{ Chapter 6} \\
\hline Cyclin D1 & 34 & 37 \\
\hline Cyclin $A 2$ & 37 & 41 \\
\hline Cyclin E & 38 & 38 \\
\hline Cyclin B1 & 41 & 41 \\
\hline$p 21 C I P$ & 37 & 37 \\
\hline p15INK & 40 & 41 \\
\hline
\end{tabular}




\section{Appendix D: Malachite green standard curve}

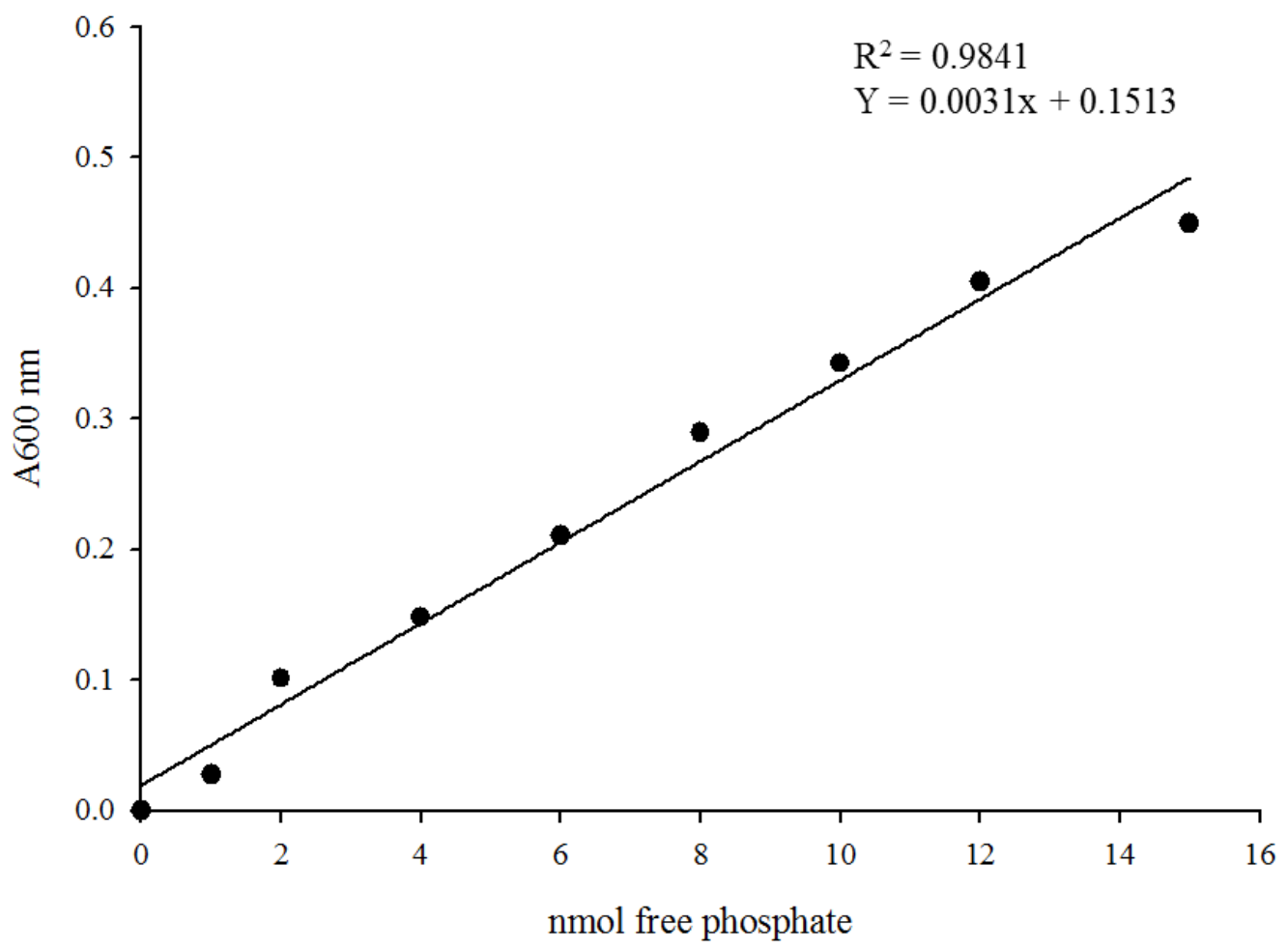




\section{Appendix E: RNA intergrity assessment (Pre-normalization of RNA concentration)}

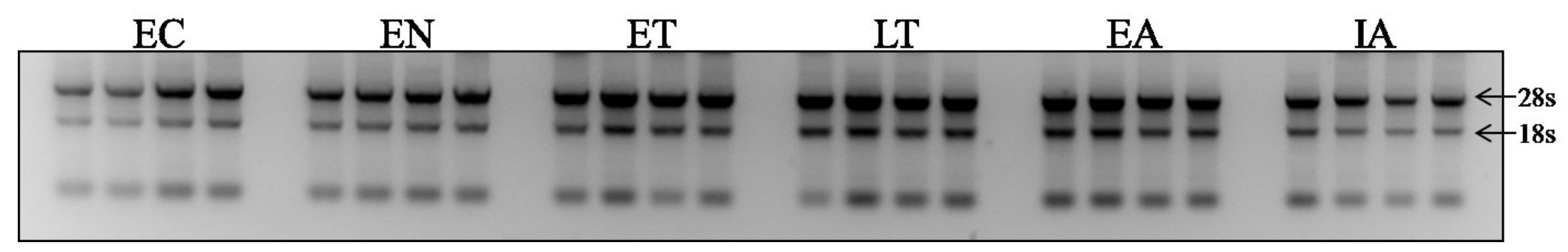




\section{Appendix F: Comparison of end-point and real time PCR analysis of ( $\alpha-$ Tubulin)}

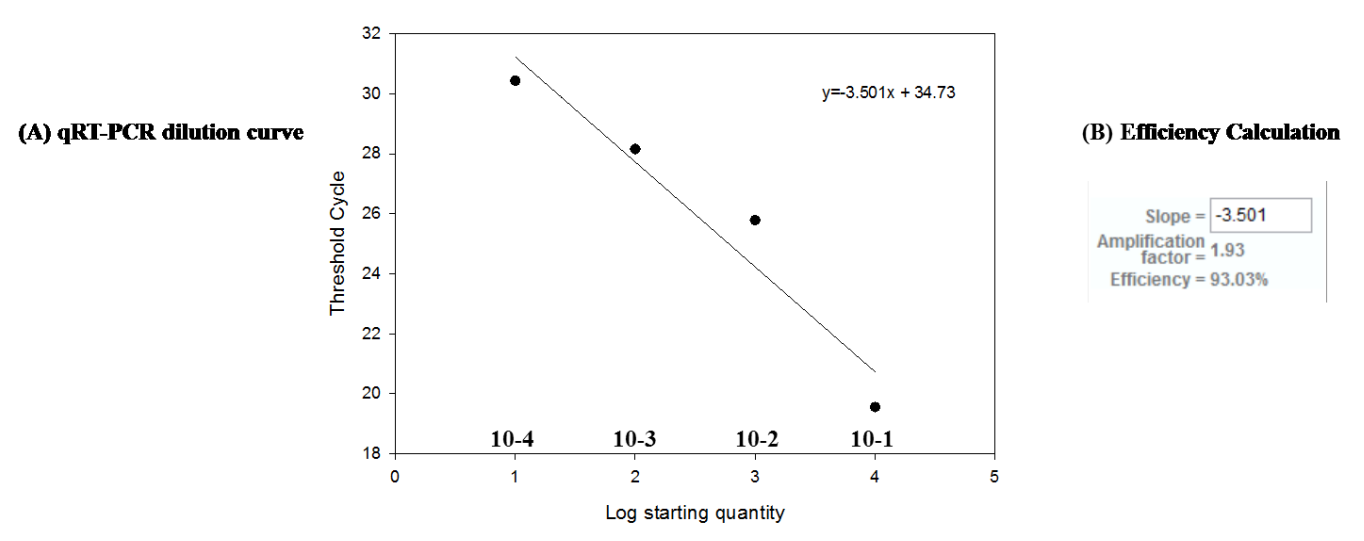

\begin{tabular}{ccc}
\multicolumn{3}{c}{ (C) Tubulin qRT-PCR quantification } \\
& $\begin{array}{c}\text { Euthermic } \\
\alpha \text {-tubulin } \mathrm{Ct}\end{array}$ & $\begin{array}{l}\text { Late torpor } \\
\alpha \text {-tubulin } \mathrm{Ct}\end{array}$ \\
\hline $\mathbf{N}=\mathbf{1}$ & 24.3 & 24.255 \\
$\mathrm{~N}=2$ & 23.74 & 23.585 \\
$\mathrm{~N}=3$ & 24.205 & 24.33 \\
$\mathrm{~N}=4$ & 22.59 & 22.635 \\
Average & 23.70875 & 23.70125 \\
& & \\
\hline Fold-Change & $=(1.93)^{(23.7087-23.7013)}=1.00049$
\end{tabular}

(D) Tubulin endpoint SYBR PCR (25 cycles)

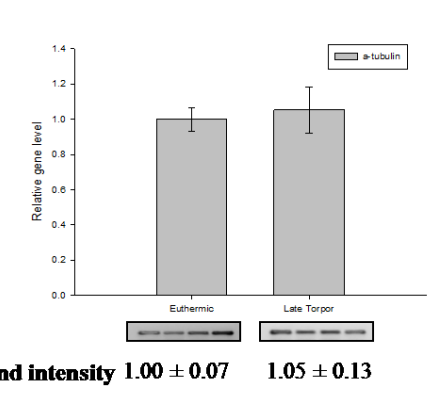

Relative band intensity $1.00 \pm 0.07 \quad 1.05 \pm 0.13$ 


\section{Appendix G: Comparison of GAPDH expression with Coomassie blue stain}

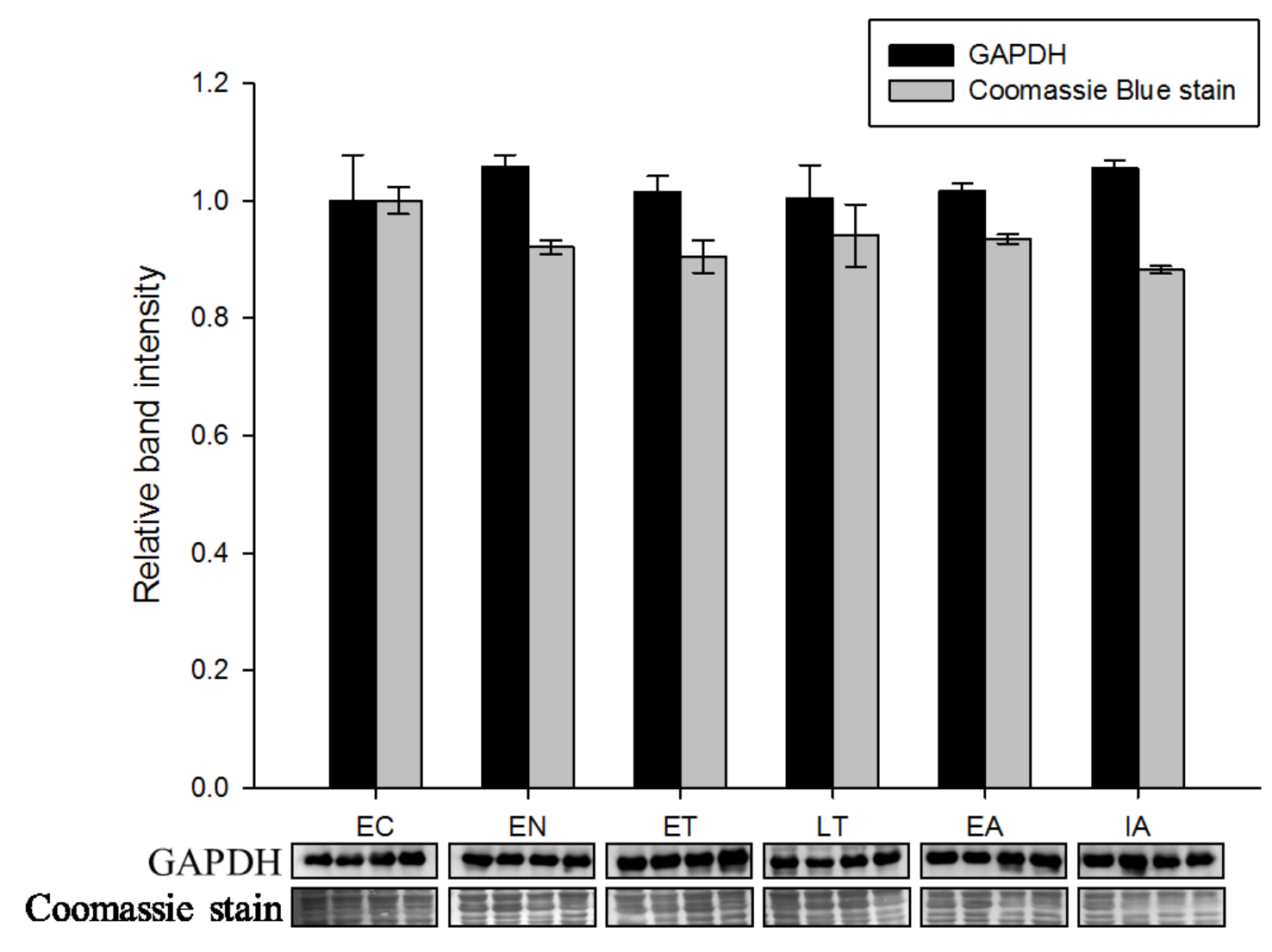




\section{REFERENCES}


1. Abnous K, Dieni CA, Storey KB. 2008. Regulation of Akt during hibernation in Richardson's ground squirrels. Biochim. Biophys. Acta 1780(2), 185-193.

2. Abnous K, Dieni CA, Storey KB. 2012. Suppression of MAPKAPK2 during mammalian hibernation. Cryobiology. 65(3):235-41.

3. Abnous K, Storey KB. 2007. Regulation of skeletal muscle creatine kinase from a hibernating mammal. Arch Biochem Biophys. 467(1):10-9.

4. Abraham RT. 2001. Cell cycle checkpoint signaling through the ATM and ATR kinases. Gene Dev. 15(17): 2177-2196.

5. Anderson RM, Weindruch R. 2010. Metabolic reprogramming, caloric restriction and aging. Trends Endocrinol Metab. 21(3):134-41.

6. Arellano M, Moreno S. 1997. Regulation of CDK/cyclin complexes during the cell cycle. Int J Biochem Cell Biol. 29(4): 559-573.

7. Averous J, Fonseca BD, Proud CG. 2008. Regulation of cyclin D1 expression by mTORC1signaling requires eukaryotic initiation factor 4E-binding protein 14EBP1 couples mTORC1 to cyclin D1 expression. Oncogene. 27: 1106-1113.

8. Avruch J. 1998. Insulin signal transduction through protein kinase cascades. 1998. Mol Cell Biochem. 182(1-2):31-48.

9. Bai H, Xu R, Cao Z, Wei D, Wang C. 2011. Involvement of miR-21 in resistance to daunorubicin by regulating PTEN expression in the leukaemia K562 cell line. FEBS Lett. 585(2):402-8.

10. Biggar KK, Storey KB. 2009. Perspective in cell cycle regulation: lessons from an anoxic vertebrate. Curr Genomics. 10:573-584.

11. Biggar KK, Storey KB. 2012. Evidence for cell cycle suppression and microRNA regulation of cyclin D1 during anoxia exposure in turtles. Cell Cycle. 11(9):170513.

12. Bloom J, Cross FR. 2007. Multiple levels of cyclin specificity in cell-cycle control. Nat Rev Mol Cell Biol. 8(2): 149-160.

13. Bodine SC, Stitt TN, Gonzalez M, Kline WO, Stover GL, Bauerlein R, Zlotchenko E, Scrimgeour A, Lawrence JC, Glass DJ, Yancopoulos GD. 2001. Akt/mTOR pathway is a crucial regulator of skeletal muscle hypertrophy and can prevent muscle atrophy in vivo. Nat Cell Biol. 3(11):1014-9.

14. Bottazzi ME, Assoian RK. 1997. The extracellular matrix and mitogenic growth factors control G1 phase and cyclins and cyclin-dependent kinase inhibitors. Trends Cell Biol. 7(9): 348-352. 
15. Brauch KM, Dhruv ND, Hanse EA, Andrews MT. 2005. Digital transcriptome analysis indicates adaptive mechanisms in the heart of a hibernating mammal. Physiol. Genom. 23(2), 227-234.

16. Brooks NE, Myburgh KH, Storey KB. 2011. Myostatin levels in skeletal muscle of hibernating ground squirrels. J. Exp. Biol. 214, 2522-2527.

17. Brooks SPJ, Storey KB. 1992. Mechanisms of glycolytic control during hibernation in the ground squirrel Spermophilus lateralis. J Comp Physiol [B]162: 23-28.

18. Brown JC, Chung DJ, Belgrave KR, Staples JF. 2012. Mitochondrial metabolic suppression and reactive oxygen species production in liver and skeletal muscle of hibernating thirteen-lined ground squirrels. Am J Physiol Regul Integr Comp Physiol. 302(1):R15-28.

19. Brugarolas J, Lei K, Hurley RL, Manning BD, Reiling JH, Hafen E, Witters LA, Ellisen LW, Kaelin WG Jr. 2004. Regulation of mTOR function in response to hypoxia by REDD1 and the TSC1/TSC2 tumor suppressor complex. Genes Dev. 18(23):2893-904.

20. Buck MJ, Squire TL, Andrews MT. 2002. Coordinate expression of the PDK4 gene: a means of regulating fuel selection in a hibernating mammal. Physiol Genomics. 8(1):5-13.

21. Cai D, McCarron RM, Yu EZ, Li Y, Hallenbeck J. 2004. Akt phosphorylation and kinase activity are down-regulated during hibernation in the 13-lined ground squirrel. Brain Res. 1014(1-2), 14-21.

22. Cánepa ET, Scassa ME, Ceruti JM, Marazita MC, Carcagno AL, Sirkin PF, Ogara MF. 2007. INK4 proteins, a family of mammalian CDK inhibitors with novel biological functions. IUBMB Life. 59(7): 419-426.

23. Carey HV, Andrews MT, Martin SL. 2003. Mammalian hibernation: cellular and molecular responses to depressed metabolism and low temperature. Physiol Rev. 83(4):1153-81.

24. Carrera AC. 2004. TOR signaling in mammals. J. Cell. Sci. 117, 4615-4616.

25. Casamayor A, Morrice NA, Alessi DR. 1999. Phosphorylation of Ser-241 is essential for the activity of 3-phosphoinositide-dependent protein kinase-1: identification of five sites of phosphorylation in vivo. Biochem J. 342 (Pt 2):28792.

26. Cayrol C, Knibiehler M, Ducommun B. 1998. p21 binding to PCNA causes G1 and G2 cell cycle arrest in p53-deficient cells. Oncogene. 12(3): 311-320. 
27. Chan JA, Krichevsky AM, Kosik KS. 2005. MicroRNA-21 is an antiapoptotic factor in human glioblastoma cells. Cancer Res. 65(14):6029-33.

28. Chan TO, Rittenhouse SE, Tsichlis PN. 1999. AKT/PKB and other D3 phosphoinositide-regulated kinases: kinase activation by phosphoinositidedependent phosphorylation. Annu Rev Biochem. 68:965-1014.

29. Cheung P, Allis CD, Sassone-Corsi P. 2000. Signaling to chromatin through histone modifications. Cell. 103(2):263-71.

30. Clausen T. 1986. Regulation of active $\mathrm{Na}+-\mathrm{K}+$ transport in skeletal muscle. Physiol Rev. 166(3):542-80.

31. Cmielová J, Rezáčová M. 2011. p21Cip1/Waf1 protein and its function based on a subcellular localization. J Cell Biochem. 112(12):3502-6.

32. Coffey RJ Jr, Bascom CC, Sipes NJ, Graves-Deal R, Weissman BE, Moses HL. 1988. Selective inhibition of growth-related gene expression in murine keratinocytes by transforming growth factor beta. Mol Cell Biol. 8(8):3088-93.

33. Coller HA, Sang L, Roberts JM. 2006. A new description of cellular quiescence. PLos Biol. 4(3): 329-347.

34. Connolly E, Braunstein S, Formenti S, Schneider RJ. 2006.Hypoxia inhibits protein synthesis through a 4E-BP1 and elongation factor 2 kinase pathway controlled by mTOR and uncoupled in breast cancer cells.Mol. Cell. Biol. 26(10): 3955- 3965.

35. Contreras A, Hale TK, Stenoien DL, Rosen JM, Mancini MA, Herrera RE. 2003. The dynamic mobility of histone $\mathrm{H} 1$ is regulated by cyclin/CDK phosphorylation. Mol Cell Biol. 23(23): 8626-8636.

36. Dark J. 2005. Annual lipid cycles in hibernators: integration of physiology and behavior. Annu Rev Nutr. 25:469-97.

37. Dausmann KH, Glos J, Ganzhorn JU, Heldmaier G. 2004. Physiology: hibernation in a tropical primate. Nature. 429(6994):825-6.

38. Davis BN, Hilyard AC, Lagna G, Hata A. 2008. SMAD proteins control DROSHA-mediated microRNA maturation. Nature. 454(7200):56-61

39. Davis-Dusenbery BN, Hata A. 2011. Smad-mediated miRNA processing: a critical role for a conserved RNA sequence. RNA Biol. 8(1):71-6. 
40. Douglas RM, Farahani R, Morcillo P, Kanaan A, Xu T, Haddad GG. 2005. Hypoxia induces major effects on cell cycle kinetics and protein expression in Drosophila melanogaster embryos. Am J Physiol Regul Integr Comp Physiol. 288(2):R511-21.

41. Eddy SF, Morin P Jr, Storey KB. 2005. Cloning and expression of PPAR-gamma and PGC-1alpha from the hibernating ground squirrel, Spermophilus tridecemlineatus. Mol Cell Biochem. 269(1-2):175-82.

42. Epperson LE, Dahl TA, Martin SL. 2004. Quantitative analysis of liver protein expression during hibernation in the golden-mantled ground squirrel. Mol Cell Proteomics. 3:920-933. Exp. Physiol. 88:141-48.

43. Fahlman A, Storey JM, Storey KB. 2000. Gene up-regulation in heart during mammalian hibernation. Cryobiology 40, 332-342.

44. Feng XH, Lin X, Derynck R. 2000. Smad2, Smad3 and Smad4 cooperate with Sp1 to induce p15Ink4B transcription in response to TGF- $\beta$. EMBO J. 19(19): 5178-5193.

45. Fielenbach N, Antebi A. 2008. C. elegans dauer formation and the molecular basis of plasticity. Genes Dev. 22(16):2149-65.

46. Florant GL, Lawrence AK, Williams K, Bauman WA. 1985. Seasonal changes in pancreatic B-cell function in euthermic yellow-bellied marmots. Am J Physiol. 249(2 Pt 2):R159-65.

47. Foster KG, Fingar DC. 2010. Mammalian target of rapamycin (mTOR): conducting the cellular signaling symphony. J. Biol. Chem. 285(19), 1407114077.

48. Frerichs KU, Smith CB, Brenner M, DeGracia DJ, Krause GS, Marrone L, Dever TE, Hallenbeck JM. 1998. Suppression of protein synthesis in brain during hibernation involves inhibition of protein initiation and elongation. Proc. Natl. Acad. Sci. USA 95(24), 14511-14516.

49. Fukuyama M, Rougvie AE, Rothman JH. 2006. C. elegans DAF-18/PTEN mediates nutrient-dependent arrest of cell cycle and growth in the germline. Curr Biol. 16(8):773-9.

50. Gallego C, Garí E, Colomina N, Herrero E, Aldea M. 1997. The Cln3 cyclin is down-regulated by translational repression and degradation during the G1 arrest caused by nitrogen deprivation in budding yeast. EMBO J. 16(23):7196-206.

51. Geiser F. 2004. Metabolic rate and body temperature reduction during hibernation and daily torpor. Annu Rev Physiol. 66:239-74. 
52. Girard F, Strausfeld U, Fernandez A, Lamb NJ. 1991. Cyclin A is required for the onset of DNA replication in mammalian fibroblasts. Cell. 67(6): 1169-1179.

53. Hay N, Sonenberg N. 2004. Upstream and downstream of mTOR. Genes Dev. 18(16), 1926-1945.

54. Heldmaier G, Ortmann S, Elvert R. 2004. Natural hypometabolism during hibernation and daily torpor in mammals. Respir Physiol Neurobiol. 141(3):31729.

55. Hinds PW, Mittnacht S, Dulic V, Arnold A, Reed SI, Weinberg RA. 1992. Regulation of retinoblastoma protein functions by ectopic expression of human cyclins. Cell.70(6): 993-1006.

56. Hirsch E, Costa C, Ciraolo E. 2007. Phosphoinositide 3-kinases as a common platform for multi-hormone signaling. J Endocrinol. 194(2):243-56.

57. Hittel D, Storey KB. 2001. Differential expression of adipose- and heart-type fatty acid binding proteins in hibernating ground squirrels. Biochim Biophys Acta. 1522(3):238-43.

58. Huang J, Manning BD. 2008. The TSC1-TSC2 complex: a molecular switchboard controlling cell growth. Biochem J 412(2): 179-190.

59. Hudder A, Novak RF. 2008. miRNAs: Effectors of environmental influences on gene expression and disease. J. Toxicol. Sci. 103(2): 228-240.

60. Humeniuk R, Rosu-Myles M, Fares J, Koller R, Bies J, Wolff L. 2013. The role of tumor suppressor p15Ink $4 \mathrm{~b}$ in the regulation of hematopoietic progenitor cell fate. Blood Cancer J. 4;2:e99.

61. Inoki K, Li Y, Zhu T, Wu J, Guan KL. 2002. TSC2 is phosphorylated and inhibited by Akt and suppresses mTOR signalling. Nat. Cell. Biol. 4(9), 648-657.

62. Inoue T, Thomas JH. 2000. Targets of TGF- $\beta$ Signalling in Caenorhabditis elegans Dauer Formation. Dev. Biol.217:192-204.

63. Iorio MV, Visone R, Di Leva G, Donati V, Petrocca F, Casalini P, Taccioli C, Volinia S, Liu CG, Alder H, Calin GA, Ménard S, Croce CM. 2007. MicroRNA signatures in human ovarian cancer. Cancer Res. 67(18):8699-707.

64. Jackman M, Lindon C, Nigg EA, Pines J. 2003. Active cyclin B1-Cdk1 first appears on centrosomes in prophase. Nat Cell Biol. 5(2): 143-148. 
65. Johnson DG, Walker CL. 1999. Cyclins and cell cycle checkpoints. Annu Rev Pharmacol Toxicol. 39: 295-312.

66. Jones SM, Kazlauskas A. 2000. Connecting signaling and cell cycle progression in growth factor-stimulated cells. Oncogene. 19(49): 5558- 5567.

67. Kagawa S, Fujiwara T, Kadowaki Y, Fukazawa T, Sok-Joo R, Roth JA, Tanaka N. 1999. Overexpression of the p21 sdil gene induces senescence-like state in human cancer cells: implication for senescence-directed molecular therapy for cancer. Cell Death Differ. 6(8): 765-772.

68. Kasahara K, Goto H, Enomoto M, Tomono Y, Kiyono T, Inagaki M. 2010. 14-33 gamma mediates Cdc25A proteolysis to block premature mitotic entry after DNA damage. EMBO J. 29(16):2802-12.

69. Katsuno Y, Suzuki A, Sugimura K, Okumura K, Zineldeen DH, Shimada M, Niida H, Mizuno T, Hanaoka F, Nakanishi M. 2009. Cyclin A-Cdk1 regulates the origin firing program in mammalian cells. Proc Natl Acad Sci U S A.106(9):3184-3189.

70. Kelly JM, McBride BW. 1990. The sodium pump and other mechanisms of thermogenesis in selected tissues. Proc. Nutr. Soc. 49(2), 185-202.

71. Kim DH, Sarbassov DD, Ali SM, Latek RR, Guntur KV, Erdjument-Bromage H, Tempst P, Sabatini DM. 2003. GbetaL, a positive regulator of the rapamycinsensitive pathway required for the nutrient-sensitive interaction between raptor and mTOR. Mol. Cell. 11(4), 895-904.

72. Kim E, Du L, Bregman DB, Warren SL. 1997. Splicing factors associate with hyperphosphorylated RNA polymerase II in the absence of pre-mRNA. J Cell Biol. 136(1):19-28.

73. Kim E, Goraksha-Hicks P, Li L, Neufeld TP, Guan KL. 2008. Regulation of TORC1 by Rag GTPases in nutrient response. Nat. Cell Biol. 10(8), 935-945.

74. Kim SH, Rowe J, Fujii H, Jones R, Schmierer B, Kong BW, Kuchler K, Foster D, Ish-Horowicz D, Peters G. 2006. Upregulation of chicken p15INK4b at senescence and in the developing brain. J Cell Sci. 119(Pt 12):2435-43.

75. Kim SH, Rowe J, Fujii H, Jones R, Schmierer B, Kong BW, Kuchler K, Foster D, Ish-Horowicz D, Peters G. 2006. Upregulation of chicken p15INK4b at senescence and in the developing brain. J Cell Sci. 119(12): 2435-2443.

76. Kisielewska J, Lu P, Whitaker M. 2005. GFP-PCNA as an S-phase marker in embryos during the first and subsequent cell cycles. Biol Cell. 97: 221-229. 
77. Klingenspor M. 2003. Cold-induced recruitment of brown adipose tissue thermogenesis.

78. Kong W, Yang H, He L, Zhao J, Coppola D, Dalton WS, Cheng JQ . 2008. MicroRNA-155 is regulated by the transforming growth factor $\beta /$ Smad pathway and contributes to epithelial cell plasticity by targeting RhoA. Mol. Cell. Biol. 28(22): 6773-6784.

79. Kovacina KS, Park GY, Bae SS, Guzzetta AW, Schaefer E, Birnbaum MJ, Roth RA. 2003. Identification of a proline-rich Akt substrate as a 14-3-3 binding partner. J. Biol. Chem. 278(12), 10189-10194.

80. Kriegel AJ, Liu Y, Fang Y, Ding X, Liang M. 2012. The miR-29 family: genomics, cell biology, and relevance to renal and cardiovascular injury. Physiol Genomics. 44(4):237-44.

81. Kurtz CC, Carey HV. 2007. Seasonal changes in the intestinal immune system of hibernating ground squirrels. Dev Comp Immunol. 31(4):415-28.

82. Lazaro JB, Bailey PJ, Lassar AB. 2002. Cyclin D-cdk4 activity modulates the subnuclear localization and interaction of MEF2 with SRC-family coactivators during skeletal muscle differentiation. Genes Dev. 16(14):1792-805.

83. Lee CH, Inoki K, Guan KL. 2007. mTOR pathway as a target in tissue hypertrophy. Annu Rev Pharmacol Toxicol. 47:443-67.

84. Lee K, Park JY, Yoo W, Gwag T, Lee JW, Byun MW, Choi I. 2008. Overcoming muscle atrophy in a hibernating mammal despite prolonged disuse in dormancy: proteomic and molecular assessment. J. Cell. Biochem. 104(2), 642-656.

85. Lee K, So H, Gwag T, Ju H, Lee JW, Yamashita M, Choi I. 2010. Molecular mechanism underlying muscle mass retention in hibernating bats: role of periodic arousal. J. Cell. Physiol. 222(2), 313-319.

86. Lee KY, Bae SC. 2002. TGF-beta-dependent cell growth arrest and apoptosis. J Biochem Mol Biol. 35(1):47-53.

87. Leung AK, Sharp PA. 2010. MicroRNA functions in stress responses. Mol Cell. 40(2):205-15.

88. Levine AJ, Oren M. 2009. The first 30 years of p53: growing ever more complex. Nat Rev Cancer. 9(10):749-58.

89. Li J, Yen C, Liaw D, Podsypanina K, Bose S, Wang SI, Puc J, Miliaresis C, Rodgers L, McCombie R, Bigner SH, Giovanella BC, Ittmann M, Tycko B, Hibshoosh H, Wigler MH, Parsons R. 1997. PTEN, a putative protein tyrosine 
phosphatase gene mutated in human brain, breast, and prostate cancer. Science. 275(5308):1943-7.

90. Li N, Cui J, Duan X, Chen H, Fan F. 2012. Suppression of type I collagen expression by miR-29b via PI3K, Akt, and Sp1 pathway in human Tenon's fibroblasts. Invest Ophthalmol Vis Sci. 53(3):1670-8.

91. Linzey AV, NatureServe (Hammerson, G.) 2008. Spermophilus tridecemlineatus. In: IUCN 2012. IUCN Red List of Threatened Species. Version 2012.2.

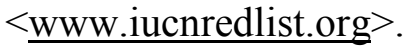

92. Lo YT, Tsao CJ, Liu IM, Liou SS, Cheng JT. 2004. Increase of PTEN gene expression in insulin resistance. Horm Metab Res. 36(10):662-6.

93. Lowell BB, Spiegelman BM. 2000. Towards a molecular understanding of adaptive thermogenesis. Nature. 404(6778):652-60.

94. Loyer P, Corlu A, Desdouets C. 2012. Regulation of the hepatocyte cell cycle: signaling pathways and protein kinases. Int J Hepatol. 2012:592354.

95. Ma XM, Blenis J. 2009. Molecular mechanisms of mTOR-mediated translational control. Nat. Rev. Mol. Cel.1 Biol. 10, 307-318.

96. MacDonald JA, Storey KB.1999. Regulation of ground squirrel $\mathrm{Na}^{+} \mathrm{K}^{+}$-ATPase activity by reversible phosphorylation during hibernation. Biochem Biophys Res Commun. 254(2); 424-429.

97. MacDonald JA, Storey KB. 2005. Mitogen-activated protein kinases and selected downstream targets display organ-specific responses in the hibernating ground squirrel. Int J Biochem Cell Biol. 37(3): 679-691.

98. Maehama T, Dixon JE. 1998. The tumor suppressor, PTEN/MMAC1, dephosphorylates the lipid second messenger, phosphatidylinositol 3,4,5trisphosphate. J Biol Chem. 273(22):13375-8.

99. Mamady H, Storey KB. 2006. Up-regulation of the endoplasmic reticulum molecular chaperone GRP78 during hibernation in thirteen-lined ground squirrels. Mol. Cell. Biochem. 292(1-2), 89-98.

100. Mandal S, Guptan P, Owusu-Ansah E, Banerjee U. 2005. Mitochondrial regulation of cell cycle progression during development as revealed by the tenured mutation in Drosophila. Dev Cell. 9(6): 843-854.

101. Manning BD, Cantley LC. 2007. AKT/PKB signaling: navigating downstream. Cell. 129(7):1261-74. 
102. Marquez RT, Bandyopadhyay S, Wendlandt EB, Keck K, Hoffer BA, Icardi MS, Christensen RN, Schmidt WN, McCaffrey AP. 2010. Correlation between microRNA expression levels and clinical parameters associated with chronic hepatitis C viral infection in humans. Lab Invest. 90(12):1727-36.

103. Martin SL, Maniero GD, Carey C, Hand SC. 1999. Reversible depression of oxygen consumption in isolated liver mitochondria during hibernation. Physiol Biochem Zool. 72(3):255-64.

104. Massagué J, 2000. How cells read TGF- $\beta$ signals. Nat. Rev. Mol. Cell. Bio. $1: 169-178$.

105. Massagué, J, Seoanne J, Wotton D. 2005. Smad transcription factors. Gene Dev. 19:2783- 2810.

106. Mathews MB, Sonenberg N, Hershey JWB. 2000. Origins and principles of translational control. In Translational control of gene expression (ed. M.B. Mathews), pp. 1-31 New York: Cold Spring Harbor Laboratory Press.

107. McArthur MD, Milsom WK. 1991. Changes in ventilation and respiratory sensitivity associated with hibernation in Columbian (Spermophilus columbianus) and golden-mantled (Spermophilus lateralis) ground squirrels. Physiol. Zool. 64, 940-959.

108. McCarley, H. 1966. Annual cycle, population dynamics and adaptive behavior of Citellus tridecemlineatus. Journal of Mammalogy, 47, 294-316.

109. McConnell BB, Gregory FJ, Stott FJ, Hara E, Peters G. 1999. Induced Expression of p16INK4a Inhibits Both CDK4- and CDK2-Associated Kinase Activity by Reassortment of Cyclin-CDK-Inhibitor Complexes. Mol Cell Biol. 19(3): 19811989.

110. McManus EJ, Alessi DR. 2002.TSC1-TSC2: a complex tale of PKB-mediated S6K regulation. Nat. Cell. Biol. 4(9), 214-216.

111. McMullen DC, Hallenbeck JM. 2010. Regulation of Akt during torpor in the hibernating ground squirrel, Ictidomys tridecemlineatus. J Comp Physiol B. 180(6):927-34.

112. McNally JD, Wu SB, Sturgeon CM, Storey KB. 2002. Identification and characterization of a novel freezing inducible gene, li16, in the wood frog Rana sylvatica. FASEB J. 16(8):902-4.

113. Merrick WC. 1992. Mechanism and regulation of eukaryotic protein synthesis. Microbiol Rev. 56(2): 291-315.

114. Meyuhas O, Avni D, Shama S. 1996. Translational control of ribosomal protein 
mRNAs in eukaryotes, In Translational Control (ed J.W.B. Hershey, M.B. Mathews, and N. Sonenberg). pp. 363-388. New York: Cold Spring Harbor Laboratory Press.

115. Mihaylova VT, Borland CZ, Manjarrez L, Stern MJ, Sun H. 1999. The PTEN tumor suppressor homolog in Caenorhabditis elegans regulates longevity and dauer formation in an insulin receptor-like signaling pathway. Proc Natl Acad Sci U S A. 96(13):7427-32.

116. Montarras D, Pinset C, Chelly J, Kahn A, Gros F. 1989. Expression of MyoD1 coincides with terminal differentiation in determined but inducible muscle cells. EMBO J. 8(8):2203-7.

117. Morin P Jr, Ni Z, McMullen DC, Storey KB. 2008. Expression of Nrf2 and its downstream gene targets in hibernating 13-lined ground squirrels, Spermophilus tridecemlineatus. Mol. Cell. Biochem. 312(1-2), 121-129.

118. Morin P Jr, Storey KB. 2006. Evidence for a reduced transcriptional state during hibernation in ground squirrels. Cryobiology 53(3), 310-318.

119. Morin P, Storey KB. 2008. Evidence for a reduced transcriptional state during hibernation in ground squirrels. Cryobiology. 53(3), 310-318.

120. Nakayama K, Nakayama K. 1998. Cip/Kip cyclin-dependent kinase inhibitors: brakes of the cell cycle engine during development. Bioessays. 20(12):1020-9.

121. Nielsen PJ, Thomas G, Maller JL. 1982. Increased phosphorylation of ribosomal protein S6 during meiotic maturation of Xenopus oocytes. Proc. Natl. Acad. Sci. U S A. 79(9), 2937-2941.

122. Nowell MM, Choi H, Rourke BC. 2011. Muscle plasticity in hibernating ground squirrels (Spermophilus lateralis) is induced by seasonal, but not lowtemperature, mechanisms. J. Comp. Physiol. B 181(1), 147-164.

123. Obaya AJ, Sedivy JM. 2002. Regulation of cyclin-Cdk activity in mammalian cells. Cell Mol Life Sci. 59: 126-142.

124. Odriozola L, Singh G, Hoang T, Chan AM. 2007. Regulation of PTEN activity by its carboxyl-terminal autoinhibitory domain. J Biol Chem. 282(32):23306-15.

125. Ohtani K, Nevins JR. 1994. Functional properties of a Drosophila homolog of the E2F1 gene. Mol Cell Biol. 14(3): 1603-1612.

126. Okita N, Minato S, Ohmi E, Tanuma S, Higami Y. 2012. DNA damage-induced CHK1 autophosphorylation at Ser296 is regulated by an intramolecular mechanism. FEBS Lett. 586(22):3974-9. 
127. Ortega-Molina A, Serrano M. 2012. PTEN in cancer, metabolism, and aging. Trends Endocrinol Metab. S1043-2760(12)00202-0.

128. Ortmann S, Heldmaier G. 2000. Regulation of body temperature and energy requirements of hibernating alpine marmots (Marmota marmota). Am J Physiol Regul Integr Comp Physiol. 278(3):R698-704.

129. Park C, Marqusee S. 2005. Pulse proteolysis: a simple method for quantitative determination of protein stability and ligand binding. Nat Methods. 2(3):207-12.

130. Park SY, Lee JH, Ha M, Nam JW, Kim VN. 2009. miR-29 miRNAs activate p53 by targeting p85 alpha and CDC42. Nat Struct Mol Biol. 16(1):23-9.

131. Pavletich NP. 1999. Mechanisms of cyclin-dependent kinase regulation: structures of Cdks, their cyclin activators, and Cip and INK4 inhibitors. J Mol Biol. 287(5):821-8.

132. Pende M, Um SH, Mieulet V, Sticker M, Goss VL, Mestan J, Mueller M, Fumagalli S, Kozma SC, Thomas G. 2004. S6K1(-/-)/S6K2(-/-) mice exhibit perinatal lethality and rapamycin-sensitive 5'-terminal oligopyrimidine mRNA translation and reveal a mitogen-activated protein kinase-dependent S6 kinase pathway. Mol Cell Biol. 24(8), 3112-3124.

133. Pestova TV, Hellen CU. 2003. Translation elongation after assembly of ribosomes on the Cricket paralysis virus internal ribosomal entry site without initiation factors or initiator tRNA. Genes Dev. 17(2), 181-186.

134. Pezzolesi MG, Platzer P, Waite KA, Eng C. 2008. Differential expression of PTEN-targeting microRNAs miR-19a and miR-21 in Cowden syndrome. Am J Hum Genet. 82(5):1141-9.

135. Pierreux CE, Nicolás FJ, Hill CS. 2000. Transforming growth factor betaindependent shuttling of Smad4 between the cytoplasm and nucleus. Mol Cell Biol. 20(23):9041-54.

136. Pullen N, Thomas G. 1997. The modular phosphorylation and activation of p70s6k. FEBS Lett. 410(1), 78-82.

137. Qian J, Ling S, Castillo AC, Long B, Birnbaum Y, Ye Y. 2012. Regulation of phosphatase and tensin homolog on chromosome 10 in response to hypoxia. Am $\mathrm{J}$ Physiol Heart Circ Physiol. 302(9):H1806-17.

138. Qiao J, Kang J, Ko TC, Evers BM, Chung DH. 2006. Inhibition of transforming growth factor-beta/Smad signaling by phosphatidylinositol 3-kinase pathway. Cancer Lett. 2006 242(2):207-14. 
139. Rabinovsky R, Pochanard P, McNear C, Brachmann SM, Duke-Cohan JS, Garraway LA, Sellers WR. 2009. p85 Associates with unphosphorylated PTEN and the PTEN-associated complex. Mol Cell Biol. 29(19):5377-88.

140. Rechsteiner M, Rogers SW. 1996. PEST sequences and regulation by proteolysis. Trends Biochem Sci. 21(7):267-71.

141. Rickards J, Kelleher MJ, Storey KB. 1987. Strategies of freeze avoidance in larvae of the goldenrod gall moth, Epiblema scudderiana: Winter profiles of a natural population. J Insect Physiol. 33:443-450.

142. Riddle, DL, Swanson MM, Albert PS. 1981. Interacting genes in dauer larva formation. Nature 290:668-671.

143. Rolfe DF, Brown GC. 1997. Cellular energy utilization and molecular origin of standard metabolic rate in mammals. Physiol Rev. 77(3):731-58.

144. Ross AH, Gericke A. 2009. Phosphorylation keeps PTEN phosphatase closed for business. Proc Natl Acad Sci U S A. 106(5):1297-8.

145. Rouble AN, Hefler J, Mamady H, Storey KB, Tessier SN. 2013. Anti-apoptotic signaling as a cytoprotective mechanism in mammalian hibernation. PeerJ 1:e29.

146. Rowles J, Scherer SW, Xi T, Majer M, Nickle DC, Rommens JM, Popov KM, Harris RA, Riebow NL, Xia J, Tsui LC, Bogardus C, Prochazka M. 1996. Cloning and characterization of PDK4 on 7q21.3 encoding a fourth pyruvate dehydrogenase kinase isoenzyme in human. J Biol Chem. 271(37):22376-82.

147. Ruggero D, Sonenberg N. 2005. The Akt of translational control. Oncogene. 24, 7426-7434.

148. Sandri M, Sandri C, Gilbert A, Skurk C, Calabria E, Picard A, Walsh K, Schiaffino S, Lecker SH, Goldberg AL. 2004. Foxo transcription factors induce the atrophy-related ubiquitin ligase atrogin-1 and cause skeletal muscle atrophy. Cell 117(3), 399-412.

149. Sarbassov DD, Guertin DA, Ali SM, Sabatini DM. 2005. Phosphorylation and regulation of Akt/PKB by the rictor-mTOR complex. Science. 307(5712):1098101.

150. Schafer KA. 1998. The cell cycle: a review. Vet Pathol. 35(6): 461-478.

151. Schmelzle T, Hall MN. 2000. TOR, a central controller of cell growth. Cell 103(2), 253-262. 
152. Schmierer B, Hill CS. 2007. TGFbeta-SMAD signal transduction: molecular specificity and functional flexibility. Nat Rev Mol Cell Biol. 8(12):970-82.

153. Scott MT, Morrice N, Ball KL. 2000. Reversible phosphorylation at the Cterminal regulatory domain of $\mathrm{p} 21$ (Wafl/Cip1) modulates proliferating cell nuclear antigen binding. J Biol Chem. 275(15): 11529-11537.

154. Seoane J, Pouponnot C, Staller P, Schader M, Eilers M, Massagué J. 2001. TGF$\beta$ influences Myc, Miz-1 and Smad to control the CDK inhibitor p15Ink4b. Nat. Cell. Biol. 3:400-408.

155. Shao C, Liu Y, Ruan H, Li Y, Wang H, Kohl F, Goropashnaya AV, Fedorov VB, Zeng R, Barnes BM, Yan J. 2010. Shotgun proteomics analysis of hibernating arctic ground squirrels. Mol. Cell. Proteomics. 9(2): 313-326.

156. Shen X, Hu PP, Liberati NT, Datto MB, Frederick JP, Wang XF. 1998. TGF-betainduced phosphorylation of Smad3 regulates its interaction with coactivator p300/CREB-binding protein. Mol Biol Cell. 9(12):3309-3319.

157. Sherr CJ, Roberts JM. 1999. CDK inhibitors: positive and negative regulators of G1-phase progression. Genes Dev. 13(12): 1501-12.

158. Shi S, Massagué J. 2003. Mechanisms of TGF- $\beta$ signalling from cell membrane to the nucleus. Cell 133:685-700.

159. Siebert R, Willers CP, Schramm A, Fosså A, Dresen IM, Uppenkamp M, Nowrousian MR, Seeber S, Opalka B. 1995. Homozygous loss of the MTSl/p16 and MTS2/p15 genes in lymphoma and lymphoblastic leukaemia cell lines. Brit J Haematol. 91: 350-354.

160. Song G, Ouyang G, Bao S. 2005. The activation of Akt/PKB signaling pathway and cell survival. J. Cell Mol. Med. 9, 59-71.

161. Song MS, Salmena L, Pandolfi PP. 2012. The functions and regulation of the PTEN tumour suppressor. Nat Rev Mol Cell Biol. 13(5):283-96.

162. South FE, House WA. 1967. Energy metabolism in hibernation. In:Mammalian Hibernation III, edited by Fisher KC, Dawe AR, Lyman CP, and Schonbaum E. Edinburgh, Scotland: Oliver \& Boyd, p. 305-324.

163. Steck PA, Pershouse MA, Jasser SA, Yung WK, Lin H, Ligon AH, Langford LA, Baumgard ML, Hattier T, Davis T, Frye C, Hu R, Swedlund B, Teng DH, Tavtigian SV. 1997.

164. Identification of a candidate tumour suppressor gene, MMAC1, at chromosome 10q23.3 that is mutated in multiple advanced cancers. Nat Genet. 15(4):356-62. 
165. Stitt TN, Drujan D, Clarke BA, Panaro F, Timofeyva Y, Kline WO, Gonzalez M, Yancopoulos GD, Glass DJ. 2004. The IGF-1/PI3K/Akt pathway prevents expression of muscle atrophy-induced ubiquitin ligases by inhibiting FOXO transcription factors. Mol Cell. 14(3):395-403.

166. Storey KB, Storey JM. 1992. Natural freeze tolerance in ectothermic vertebrates. Annu Rev Physiol. 54:619-37.

167. Storey KB, Storey JM. 2004. Mammalian hibernation: biochemical adaptation and gene expression. In: Functional Metabolism, Regulation and Adaptation. (Storey, K.B., ed.) New Jersey: Wiley-Liss. pp. 443-471.

168. Storey KB, Storey JM. 2004. Metabolic rate depression in animals: transcriptional and translational controls. Biol Rev Camb Philos Soc 79:207-233.

169. Storey KB, Storey JM. 2007. Putting life on 'pause' - molecular regulation of hypometabolism. J Exp Biol. 210: 1700-1714.

170. Storey KB, Storey JM. 2007. Tribute to P.L. Lutz: Putting life on 'pause' molecular regulation of hypometabolism. J. Exp. Biol. 210, 1700-1714.

171. Storey KB, Storey JM. 2010. Metabolic rate depression: the biochemistry of mammalian hibernation. Adv Clin Chem. 52: 77-108. .

172. Storey KB. 1997. Metabolic regulation in mammalian hibernation: enzyme and protein adaptations. Comp Biochem Physiol A Physiol. 118(4):1115-24.

173. Storey KB. 1999. Living in the cold: freeze-induced gene responses in freezetolerant vertebrates. Clin Exp Pharmacol Physiol. 26(1):57-63.

174. Storey KB. 2010. Out cold: biochemical regulation of mammalian hibernation - a mini-review. Gerontology. 56(2):220-30.

175. Storey KB. 2012. Hypometabolism and the cell cycle. Cell Cycle. 11(9):1665.

176. Taub R. 2004. Liver regeneration: from myth to mechanism. Nat Rev Mol Cell Biol. 5(10): 836-847.

177. Ten Dijke P, Goumans MJ, Itoh F, Itoh S. 2002. Regulation of cell proliferation by Smad proteins. J Cell Physiol. 191(1):1-16.

178. Tessier SN, Storey KB. 2012. Myocyte enhancer factor-2 and cardiac muscle gene expression during hibernation in thirteen-lined ground squirrels. Gene. 501(1):816. 
179. Tøien Ø, Blake J, Edgar DM, Grahn DA, Heller HC, Barnes BM. 2011. Hibernation in black bears: independence of metabolic suppression from body temperature. Science. 331(6019):906-9.

180. Tokunaga C, Yoshino K, Yonezawa K. 2004. mTOR integrates amino acid- and energy sensing pathways. Biochem. Biophys. Res. Commun. 313(2), 443-446.

181. Torres J, Pulido R. 2001. The tumor suppressor PTEN is phosphorylated by the protein kinase $\mathrm{CK} 2$ at its $\mathrm{C}$ terminus. Implications for PTEN stability to proteasome-mediated degradation. J Biol Chem. 276(2):993-8.

182. van Breukelen F, Martin SL. 2001. Translational initiation is uncoupled from elongation at 18 degrees $\mathrm{C}$ during mammalian hibernation. Am J Physiol Regul Integr Comp Physiol. 281(5):R1374-9.

183. van Breukelen F, Martin SL. 2002. Reversible depression of transcription during hibernation. J Comp Physiol B. 172(5):355-61.

184. van Breukelen F, Sonenberg N, Martin SL. 2004. Seasonal and state-dependent changes of eIF4E and 4E-BP1 during mammalian hibernation: implications for the control of translation during torpor. Am. J. Physiol. Regul. Integr. Comp. Physiol. 287(2), 349-353.

185. van den Heuvel, S. Cell-cycle regulation (September 21, 2005), WormBook, ed. The C. elegans Research Community, WormBook, doi/10.1895/wormbook.1.28.1, http://www.wormbook.org.

186. Vander Haar E, Lee SI, Bandhakavi S, Griffin TJ, Kim DH. 2007. Insulin signalling to mTOR mediated by the Akt/PKB substrate PRAS40. Nat. Cell. Biol. 9(3), 316-323.

187. Vazquez F, Ramaswamy S, Nakamura N, Sellers WR. 2000. Phosphorylation of the PTEN tail regulates protein stability and function. Mol Cell Biol. 20(14):50108.

188. Vazquez F, Grossman SR, Takahashi Y, Rokas MV, Nakamura N, Sellers WR. 2001. Phosphorylation of the PTEN tail acts as an inhibitory switch by preventing its recruitment into a protein complex. J Biol Chem. 276(52):48627-30.

189. Vermeulen K, Van Bockstaele DR, Berneman ZN. 2003.The cell cycle: a review of regulation, deregulation and therapeutic targets in cancer. Cell Prolif. 36: 131149.

190. Vidal A, Koff A. 2000. Cell-cycle inhibitors: three families united by a common cause. Gene. 247(1-2): 1-15. 
191. Volinia S, Calin GA, Liu CG, Ambs S, Cimmino A, Petrocca F, Visone R, Iorio M, Roldo C, Ferracin M, Prueitt RL, Yanaihara N, Lanza G, Scarpa A, Vecchione A, Negrini M, Harris CC, Croce CM. 2006. A microRNA expression signature of human solid tumors defines cancer gene targets. Proc Natl Acad Sci U S A. 103(7):2257-61.

192. Walker DH, Maller, JL. 1991. Role for cyclin A in the dependence of mitosis on completion of DMA replication. Nature. 354(6351): 314-317.

193. Wang C, Bian Z, Wei D, Zhang JG. 2011. miR-29b regulates migration of human breast cancer cells. Mol Cell Biochem. 352(1-2):197-207.

194. Wang D. 2008. Discrepancy between mRNA and protein abundance: insight from information retrieval process in computers. Comput Biol Chem. 32(6):462-8.

195. Wang L, Harris TE, Lawrence JC Jr. 2008. Regulation of proline-rich Akt substrate of $40 \mathrm{kDa}$ (PRAS40) function by mammalian target of rapamycin complex 1 (mTORC1)-mediated phosphorylation. J. Biol. Chem. 283(23), 1561915627.

196. Wang LCH, Wolowyk MW. 1987. Topor in mammals and birds. Can. J. Zool. 66, 133-137.

197. Wang P, Zou F, Zhang X, Li H, Dulak A, Tomko RJ Jr, Lazo JS, Wang Z, Zhang L, Yu J. 2009. microRNA-21 negatively regulates Cdc25A and cell cycle progression in colon cancer cells. Cancer Res. 69(20):8157-65.

198. Wang X ,Proud CG. 2006. The mTOR pathway in the control of protein synthesis. Physiology 21, 362-369.

199. Ward PS, Thompson CB. 2012. Metabolic reprogramming: a cancer hallmark even warburg did not anticipate. Cancer Cell. 21(3):297-308.

200. Wickler SJ, Hoyt DF, van Breukelen F. 1991. Disuse atrophy in the hibernating golden-mantled ground squirrel, Spermophilus lateralis. Am. J. Physiol. 261(5 Pt 2), 1214-1217.

201. Wolff L, Bies J. 2013. p15Ink4b Functions in determining hematopoietic cell fates: Implications for its role as a tumor suppressor. Blood Cells Mol Dis. S10799796(13)00007-7.

202. Wu CW, Reardon AJ, Storey KB. 2013. Effects of hibernation on regulation of mammalian protein phosphatase type-2-A. Cryobiology. 66(3):267-274.

203. Wu JW, Hu M, Chai J, Seoane J, Huse M, Li C, Rigotti DJ, Kyin S, Muir TW, Fairman R, Massagué J, Shi Y. 2001. Crystal structure of a phosphorylated 
Smad2. Recognition of phosphoserine by the MH2 domain and insights on Smad function in TGF-beta signaling. Mol Cell. 8(6):1277-89.

204. Yan J, Barnes BM, Kohl F, Marr TG. 2008. Modulation of gene expression in hibernating arctic ground squirrels. Physiol. Genomics 32, 170-181.

205. Yang Q, Guan KL. 2007. Expanding mTOR signaling. Cell Res. 17, 666-681

206. Zatzman ML. 1984. Renal and cardiovascular effects of hibernation and hypothermia. Cryobiology. 21(6):593-614.

207. Zhang H, Akman HO, Smith EL, Zhao J, Murphy-Ullrich JE, Batuman OA. 2003. Cellular response to hypoxia involves signaling via Smad proteins. Blood. 101(6):2253-60.

208. Zhang S, Tang Q, Xu F, Xue Y, Zhen Z, Deng Y, Liu M, Chen J, Liu S, Qiu M, Liao Z, Li Z, Luo D, Shi F, Zheng Y, Bi F. 2009. RhoA regulates G1-S progression of gastric cancer cells by modulation of multiple INK4 family tumor suppressors. Mol Cancer Res. 7(4):570-80.

209. Zhang XC, Piccini A, Myers MP, Van Aelst L, Tonks NK. 2012. Functional analysis of the protein phosphatase activity of PTEN. Biochem J. 444(3):457-64. doi: 10.1042/BJ20120098.

210. Zhang Y, Chang C, Gehling DJ, Hemmati-Brivanlou A, Derynck R. 2001. Regulation of Smad degradation and activity by Smurf2, an E3 ubiquitin ligase. Proc Natl Acad Sci U S A. 98(3):974-9.

211. Zhao H, Dupont J, Yakar S, Karas M, LeRoith D. 2004. PTEN inhibits cell proliferation and induces apoptosis by downregulating cell surface IGF-IR expression in prostate cancer cells. Oncogene. 23(3):786-94.

212. Zhao HW, Haddad GG. 2011. Review: Hypoxic and oxidative stress resistance in Drosophila melanogaster. Placenta. 32 Suppl 2:S104-8.

213. Zhegunov GF, Mikulinsky YE, Kudokotseva EV. 1988. Hyperactivation of protein synthesis in tissues of hibernating animals on arousal. Cryo-Lett 9: 236245. 